\title{
Storage solutions
}

Citation for published version (APA):

Walenbergh, S. M. A. (2016). Storage solutions: novel ways for the detection and inhibition of nonalcoholic steatohepatitis. [Doctoral Thesis, Maastricht University]. Maastricht University. https://doi.org/10.26481/dis.20160129sw

Document status and date:

Published: 01/01/2016

DOI:

10.26481/dis.20160129sw

Document Version:

Publisher's PDF, also known as Version of record

\section{Please check the document version of this publication:}

- A submitted manuscript is the version of the article upon submission and before peer-review. There can be important differences between the submitted version and the official published version of record.

People interested in the research are advised to contact the author for the final version of the publication, or visit the DOI to the publisher's website.

- The final author version and the galley proof are versions of the publication after peer review.

- The final published version features the final layout of the paper including the volume, issue and page numbers.

Link to publication

\footnotetext{
General rights rights.

- You may freely distribute the URL identifying the publication in the public portal. please follow below link for the End User Agreement:

www.umlib.nl/taverne-license

Take down policy

If you believe that this document breaches copyright please contact us at:

repository@maastrichtuniversity.nl

providing details and we will investigate your claim.
}

Copyright and moral rights for the publications made accessible in the public portal are retained by the authors and/or other copyright owners and it is a condition of accessing publications that users recognise and abide by the legal requirements associated with these

- Users may download and print one copy of any publication from the public portal for the purpose of private study or research.

- You may not further distribute the material or use it for any profit-making activity or commercial gain

If the publication is distributed under the terms of Article $25 \mathrm{fa}$ of the Dutch Copyright Act, indicated by the "Taverne" license above, 


\title{
Storage solutions:
}

\author{
Novel ways for the detection and \\ inhibition of non-alcoholic steatohepatitis
}


(C) Sofie Walenbergh, Maastricht 2015

Layout: Tiny Wouters

Cover design: Davy Nicolaes

Production: Ipskamp Printing, Enschede

ISBN: 978-94-6259-971-0
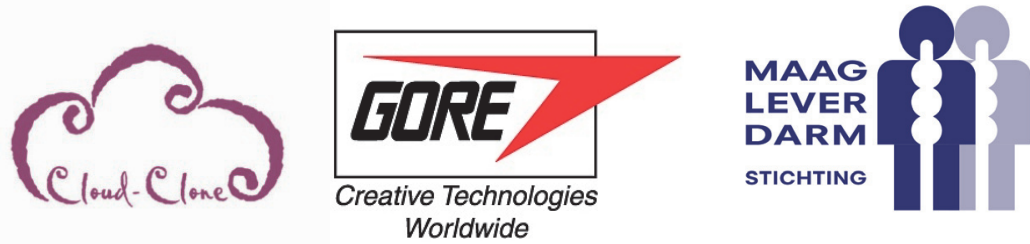

Printing of this thesis was financially supported by Cloud-Cone Corp. Wuhan, Bio-Connect Diagnostics B.V., W.L. Gore \& Associates, AbbVie, Astellas Pharma B.V., Kupffer cell foundation, Maag Lever Darm Stichting, Nederlandse Vereniging voor Hepatologie.

Financial support by the Dutch Heart Foundation for the publication of this thesis is gratefully acknowledged. 


\title{
Storage solutions:
}

\section{Novel ways for the detection and inhibition of non-alcoholic steatohepatitis}

\author{
PROEFSCHRIFT
}

ter verkrijging van de graad van doctor aan de Universiteit Maastricht, op gezag van de Rector Magnificus, Prof. dr. L.L.G. Soete, volgens het besluit van het College van Decanen, in het openbaar te verdedigen op 29 januari 2016 om 14:00 uur

door

\section{Sofie Marie Anne Walenbergh}

Geboren te Roermond op 14 september 1987 


\section{Promotor:}

Prof. dr. A.A.M. Masclee

\section{Copromotors:}

Dr. R. Shiri-Sverdlov

Dr. G.H. Koek

\section{Beoordelingscommissie:}

Prof. dr. A.M.W.J. Schols (voorzitster)

Prof. dr. B. Staels (University of Lille, France)

Prof. dr. D. Cassiman (University of Leuven, Belgium)

Prof. dr. P.L.M. Jansen 


\section{Contents}

$\begin{array}{lll}\text { Chapter } 1 \quad \text { Introduction } & 7\end{array}$

Chapter 2 Non-alcoholic steatohepatitis: the role of oxidized 21 low-density lipoproteins

Journal of Hepatology, 2013

Chapter 3 Trapping of oxidized LDL in lysosomes of Kupffer cells is a trigger of hepatic inflammation

Liver International, 2013

Chapter $4 \quad$ Lysosomal cholesterol in Kupffer cells, particularly when oxidized, contributes to murine steatohepatitis Submitted

Chapter $5 \quad$ Specific immunization strategies against oxidized low-density lipoprotein: a novel way to reduce non-alcoholic steatohepatitis in mice

Hepatology, 2012

Chapter 6 Plasma cathepsin D levels: a novel tool to predict pediatric hepatic inflammation

The American Journal of Gastroenterology, 2015

Chapter 7 Plasma cathepsin D correlates with histological 109 classifications of fatty liver disease in adults and responds to intervention

Submitted

Chapter 8 Lysosomal cholesterol accumulation: driver on the road to inflammation during atherosclerosis and non-alcoholic steatohepatitis

Obesity Reviews, 2014

Chapter 9 General discussion

Summary

Samenvatting

Valorisation

Dankwoord

Curriculum Vitae 

Chapter 1

General introduction 
Chapter 1 


\section{The metabolic syndrome}

The metabolic syndrome (MetS) refers to a cluster of interconnected risk factors that will increase the overall risk for the development of cardiovascular disease (CVD), stroke, type 2 diabetes mellitus and fatty liver disease. The main principle behind the MetS is an imbalance between energy intake and energy utilization, disrupting wholebody metabolism. The combination of the environment as well as genetic predisposition significantly contributes to the development of the MetS. Environmental factors include a sedentary lifestyle and on a nutritional-area, overnutrition of low-fibre, fat-containing and high-caloric foods. ${ }^{1}$

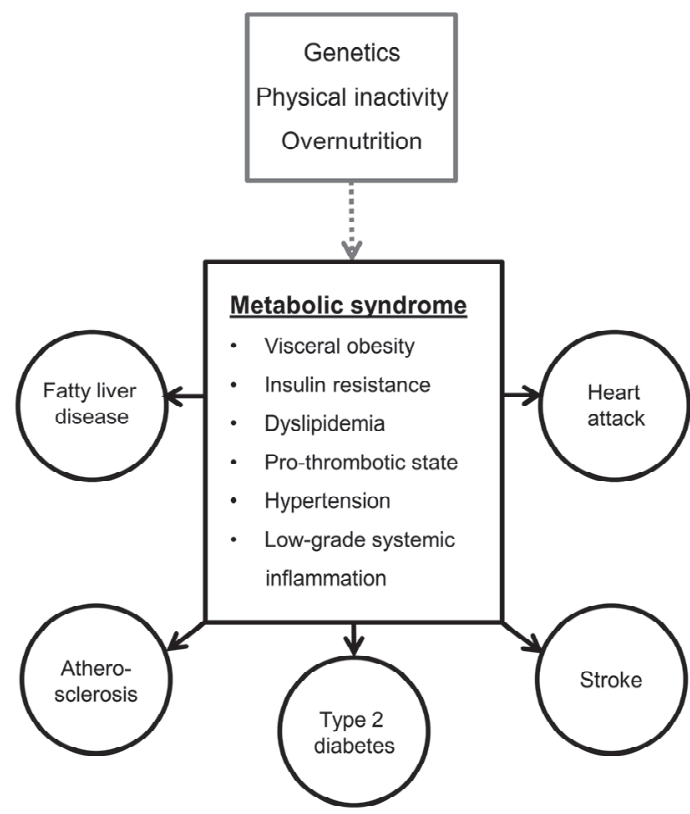

Figure 1.1 The metabolic syndrome (MetS). The MetS can be defined as a disorder in which overnutrition of predominantly fat-rich foods, physical inactivity and genetic factors result in a metabolic imbalance. Consequently, certain metabolic risk factors including obesity, dyslipidemia, and insulin resistance start to develop. The combination of obesity with two or more risk factors increases the risk for several diseases such as fatty liver disease, type 2 diabetes and stroke.

Individual risk factors that are strongly associated with the MetS include central obesity, raised triglyceride levels, reduced high-density lipoprotein (HDL, the 'good' cholesterol) levels, hypertension and an increased blood sugar level. According to the International Diabetes Foundation (IDF), individuals that are identified with the MetS must meet the criteria of having central obesity plus two additional, of the abovementioned, risk factors. ${ }^{2}$ Based on these criteria, including those of IDF, approximately 
one-fourth of the adults in Europe can be defined as having the MetS. ${ }^{3}$ Nowadays, there has been a greater concern about the prevalence of the MetS in children, which is expected to rise to epidemic proportions. The main concern is that the MetS is an important risk factor for the development of CVD, a heart attack, type 2 diabetes mellitus and fatty liver disease (Figure 1.1). ${ }^{4}$ Within the cluster of the MetS, the content of this thesis will primarily focus on the diet-induced effect on the liver.

\section{The liver is an essential metabolic organ}

The liver, which is one of the largest organs in the body, fulfills numerous functions including detoxification of many toxins including medication, drugs and alcohol. As such, the liver plays a major role in whole-body metabolism. Furthermore, the liver controls lipid metabolism, provides nutrient storage, supports immunity, assists in digestion processes through bile production and produces proteins necessary for blood plasma. $^{5}$

Approximately $20 \%$ of the total blood flow passes through the liver every minute. ${ }^{5,6}$ Unlike other organs, the liver receives blood supply from two sources being, nutrientrich blood from the intestines (portal venous system) and oxygen-rich blood from the heart. Considering its functions, the liver can be referred to as a 'first pass' organ, filtering nutrient-rich blood directly originating from the stomach and the intestines. In this way, toxic substances and pathogens can be destroyed, while other substances, such as lipids, are stored in the liver.

Lipids are fat-like substances that are either absorbed from food or synthesized by the liver and serve as a source for energy. In the blood, mainly two types of lipids are circulating, triglycerides and cholesterol. Both lipids are physiologically important, whereas triglycerides are used to store energy in muscle and fat tissue, cholesterol is essential for structural components of the cell (cell membrane) and bile acids. As lipids are not soluble in water, they require packaging into hydrophilic structures, called lipoproteins, for transport through the bloodstream. Based on their size, density and content, lipoproteins are classified as chylomicrons, very-low-density lipoproteins (VLDL), low-density lipoproteins (LDL) and high-density lipoproteins (HDL).

There are three pathways that are responsible for the metabolism of lipoproteins, the exogenous, endogenous and the reverse cholesterol transport pathway (Figure 1.2). The exogenous pathway starts with intestinal absorption of fats (triglycerides and cholesterol), which are packaged into chylomicrons. Subsequently, chylomicrons enter the circulation and travel to peripheral tissues, such as muscle and fat. By use of the endothelial-bound enzyme, lipoprotein lipase, the packaged fats into chylomicrons are converted into glycerol and free fatty acids and delivered to muscle and fat for 
energy purposes or storage. After delivery, the chylomicrons, now called chylomicron remnants, are left with carrying mainly cholesterol and circulate back to the liver for further processing.

The endogenous pathway deals with the formation of lipoproteins, mainly VLDL, in the liver. VLDL lipoproteins are loaded with fats and subsequently released into the blood. Similar to chylomicrons, VLDL particles continuously circulate through the blood until the peripheral tissues, principally muscle and fat, have taken up all the free fatty acids. The majority of the remaining VLDL remnants are cleared by the liver to become intermediate-density lipoproteins (IDL). While some IDL particles are reabsorbed by the liver, some IDL particles are incorporated into LDL lipoproteins. ${ }^{5}$ About $40-60 \%$ of these LDL particles, highly rich in cholesterol, are cleared by the liver via hepatic LDL receptors. The remaining LDL particles are absorbed by the extrahepatic, peripheral tissue.

The third route, the reverse cholesterol transport pathway is a process opposite to the exogenous and endogenous pathway. HDL particles are initially cholesterol-free particles that are synthesized by both intestinal enterocytes and the liver. Once in the blood, HDL particles promote cholesterol efflux and bind the released cholesterol. The cholesterol-carrying HDL particles return to the liver and are either reused for assembly of other lipoproteins or are excreted in the stool. As a result, HDL formation is believed to be protective for peripheral tissues as it clears cholesterol. ${ }^{5}$

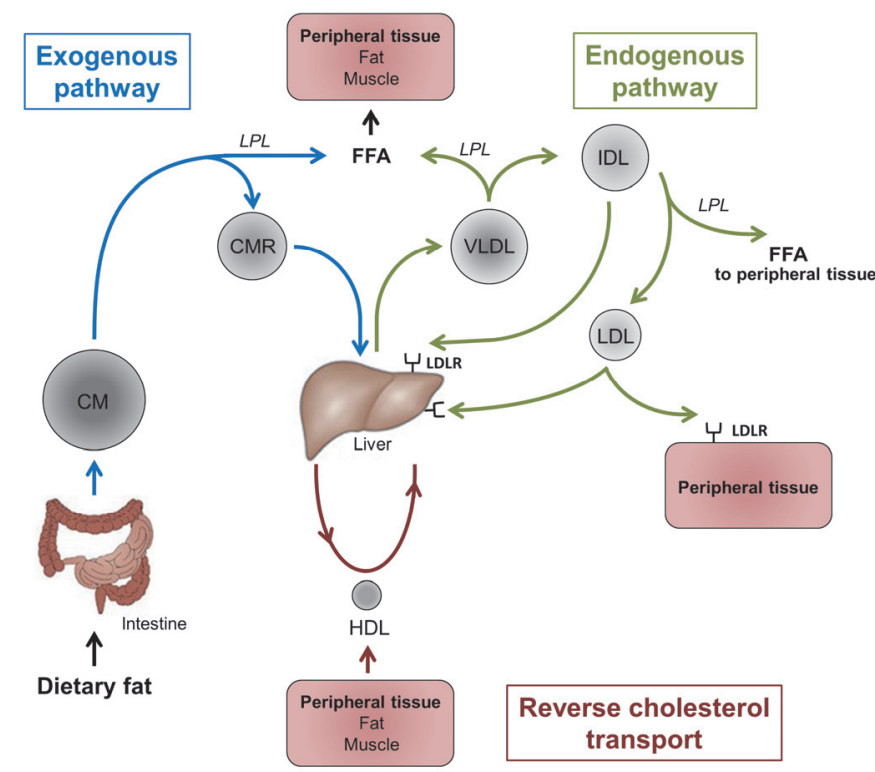

Figure 1.2 Lipoprotein metabolism. A schematic overview of the exogenous, endogenous, and reverse cholesterol transport pathway. CM: chylomicrons; CMR: chylomicron remnants; LPL: lipoprotein lipase; FFA: free fatty acids; VLDL: very low-density lipoprotein; IDL: intermediatedensity lipoprotein; LDL(R): low-density lipoprotein (receptor); HDL: high-density lipoprotein. 
Relevantly, fat-rich foods and overnutrition can cause dysregulation of these three pathways; lipoprotein synthesis, processing and clearance. As a result, there is a shift in the plasma lipoprotein profile to profoundly higher levels of triglycerides and LDL particles and lower amounts of HDL particles, called dyslipidemia. Under normal circumstances, the uptake of LDL occurs by LDL receptor-mediated endocytosis. However, under fat-rich conditions and thus high plasma LDL levels, LDL is internalized via scavenger receptor-mediated macrophage uptake in an unlimited fashion. As such, the increased amount of LDL lipoproteins predominantly starts to accumulate in the liver, which leads to a specific liver disease pathology known as non-alcoholic fatty liver disease.

\section{Non-alcoholic steatohepatitis (NASH) is a crucial hepatic manifestation of non-alcoholic fatty liver disease (NAFLD)}

The MetS is an important risk factor for the development of non-alcoholic fatty liver disease (NAFLD) and therefore NAFLD is often referred to as the hepatic event of the MetS. NAFLD encompasses a spectrum of several diet-induced liver pathologies with minimal or no alcohol consumption (ideally $<10 \mathrm{~g} /$ day for females and $<20 \mathrm{~g} /$ day for males according to the NASH CRN database). ${ }^{7,8}$ The most simple and benign form of NAFLD is steatosis, which is characterized by an excessive amount of fat accumulation in the liver due to a diet rich in fat. A more advanced liver condition of NAFLD is nonalcoholic steatohepatitis (NASH) and represents a combination of fat accumulation in the liver along with inflammation. Whereas steatosis is considered to be benign and reversible, it is the inflammatory component of NASH that increases the chance of developing severe complicated liver disease such as fibrosis, cirrhosis and ultimately liver failure or hepatocellular carcinoma. ${ }^{9}$ Thus, hepatic inflammation during NASH is a key step in the etiology of NAFLD.

\section{Epidemiology}

The prevalence of NASH worldwide is currently under investigation, as such, the precise number of individuals with NASH remains largely unknown, partly because accurate non-invasive markers to detect NASH are lacking. In general it is estimated that approximately $12 \%$ of US middle-aged adults have $\mathrm{NASH}^{10}$ with a higher NASH prevalence among Hispanics. ${ }^{10,11}$ However, in selected populations, i.e. these patients were selected to undergo a liver biopsy based on abnormal liver enzyme levels, the prevalence of NASH increased to around $34-40 \% .{ }^{12,}{ }^{13}$ Patients who were extremely overweight and underwent bariatric surgery, the prevalence was estimated to be 37\%. ${ }^{14}$ Data from the National Health and Nutrition Examination Survey III (NHANES III) demonstrated that NAFLD patients with NASH, compared to NAFLD patients without $\mathrm{NASH}$, were more likely to have visceral obesity $(88.7 \%)$, hyper- 
cholesterolemia (90.2\%) and hypertension (45.5\%). ${ }^{11}$ Thus, factors of the MetS are strong risk predictors for the development of NASH. However, it is also important to note that, unlike most NASH patients, in some cases NASH is also prevalent in healthy lean individuals. ${ }^{15}$

$\mathrm{NASH}$ is described in individuals at all ages, and becomes more common in children, especially when obese. In line with NASH in adults, this disease in children is also strongly associated with features of the MetS. Based on biopsy-proven cohorts, the prevalence of pediatric NASH is estimated to range between $68-84 \%$ in children diagnosed with a fatty liver. ${ }^{16}$ The development of NASH at a young age can have severe consequences later in life, with progression into complicated liver disease and a shorter life expectancy. ${ }^{17}$

In 2012, more than one-third of the children and adults in the US were overweight or obese. ${ }^{18}$ As it is expected that the obesity epidemic will further continue, the number of patients with NASH is expected to rise dramatically. As such, NASH is about to become a worldwide health concern and needs adequate diagnosis and therapy.

\section{NASH diagnosis}

NAFLD and NASH are often underdiagnosed due to the non-specific nature of symptoms and the lack of sensitive non-invasive screening methods. Although some patients experience pain in the upper right area of their abdomen and suffer from fatigue $^{19}$, the majority of patients have no complaints. Upon first suspicion of NAFLD and $\mathrm{NASH}$, the levels of plasma liver enzymes, alanine amino transaminase (ALAT) and aspartate amino transaminase (ASAT), are primarily assessed. Elevation of these liver enzymes in the blood points toward liver cell damage. However, cautious interpretation is required as various other liver diseases can also lead to an abnormal aminotransferase pattern ${ }^{20}$, while some NAFLD patients show aminotransferase levels in the normal range. ${ }^{21}$ In general, plasma aminotransferases are not able to distinguish patients with steatosis from those who suffer from NASH. In case aminotransferases are not conclusive, several imaging methods are a useful tool in NAFLD diagnosis. Ultrasound elastography ${ }^{22}$, computed tomography $(\mathrm{CT})^{23}$, magnetic resonance imaging $(\mathrm{MRI})^{24}$ and magnetic resonance spectroscopy (MRS) ${ }^{25}$ are noninvasive diagnostic tools to define structural abnormalities of the liver, including a fatty liver and fibrosis, however, these techniques lack the ability to detect the inflammatory component of $\mathrm{NASH} .{ }^{26}$ The most decisive, and simultaneously, the most invasive method, which is currently regarded as the gold standard to diagnose NASH, is a liver biopsy. Over the years, several histological scoring systems have been developed for diagnosing NASH in children and adults. All scoring systems basically use the same principles for NASH diagnosis and include a mixture of several liver pathologic features (steatosis, hepatocyte ballooning, lobular inflammation, portal inflammation and fibrosis), rather than assessing hepatic inflammation solely. ${ }^{27-29}$ 
Unfortunately, there are some important disadvantages to liver biopsies, including risk for complications such as pain and bleeding, patient stress and discomfort. ${ }^{30}$ Furthermore, NASH lesions are not equally distributed throughout the whole liver. As a needle biopsy represents only a small fraction of the liver (1/50.000), NASH pathology can be missed easily and can potentially result in misdiagnosis. ${ }^{31}$ Due to these reasons, a liver biopsy is not the most suitable method in the detection and clinical follow-up of NASH patients and should be replaced by specific and sensitive non-invasive markers.

\section{NASH therapy}

Currently, no approved treatment exists that is able to specifically cure and reduce $\mathrm{NASH}$. To date, simple lifestyle modifications, such as body weight management and appropriate nutritional counseling (with and without regular physical exercise and cognitive-behavior programs), were proven to be the most effective therapy against NASH. ${ }^{32,33}$ For morbidly obese patients, lifestyle changes alone are not sufficient to achieve sustained weight loss. For these subjects bariatric surgery can be considered, which has been shown to improve overall physical health and ameliorate NASH histology. ${ }^{34}$ In addition to lifestyle changes, pharmacological interventions, aimed at targeting features accompanied with NASH including hyperlipidemia, insulin resistance or oxidative stress, are also utilized. ${ }^{35}$ The use of ursodeoxycholic acid $\left(\right.$ UDCA $^{36}$, pentoxifylline ${ }^{37}$, probiotics ${ }^{38,39}$, incretins ${ }^{40,41}$ and farnesoid X receptor (FXR) agonists $^{42}$ are currently under investigation for the treatment of NASH. However, none of these therapies proved convincingly to reverse $\mathrm{NASH}$, therefore there is a need for novel therapeutic strategies against hepatic inflammation. New insights obtained from animal models are needed to find the underlying mechanisms of the pathogenesis of NASH. These findings have the potential to be developed into accurate diagnostic applications for the detection of human NASH and interventions for treatment of this disease.

\section{Hyperlipidemic $L_{d}{ }^{-/-}$mice as a suitable mouse model to study NASH}

Most animal models used to study NASH are very different from human NASH patients. For example, some genetically modified mice often need a second external stimulus, such as lipopolysaccharide, to fully develop hepatic inflammation whereas some nutritional models do not develop typical features related to the metabolic syndrome, such as hyperlipidemia and obesity. ${ }^{43}$ In the current thesis, we used mice that lacked the low-density lipoprotein receptor $\left(L d l r^{-/}\right)$, a gene important in cholesterol uptake. Upon a high-fat, high-cholesterol (HFC) diet, the $\mathrm{Ldlr}^{-1-}$ mice demonstrated mildly elevated cholesterol levels, with most cholesterol carried in the 
IDL/LDL fractions. This plasma cholesterol profile is comparable to those found in humans with an LDLR deficiency, in which cholesterol is mainly confined to the LDL fraction. ${ }^{44}$ Additional research demonstrated that hepatic inflammation persisted for a long period of time in $\mathrm{Ld}_{\mathrm{I}} \mathrm{r}^{-/}$mice, which is most likely the consequence of an increased sensitivity to the oxidized form of LDL (oxLDL). ${ }^{45}$ The resemblance with a human-like lipoprotein profile and the sustained hepatic inflammatory response makes hyperlipidemic $\mathrm{Ldll}^{-/}$mice a suitable mouse model that can be used to study the onset and progression of NASH.

\section{Lysosomal cholesterol accumulation inside macrophages is associated with intracellular trafficking defects}

Kupffer cells (KCs), the liver macrophages, are considered to be in the driver's seat during the disease process of NASH. ${ }^{46-48}$ Upon HFC feeding of $\mathrm{Ldll}^{-/-}$mice, we have found that KCs are essential in scavenging modified lipids, such as OxLDL, via the socalled scavenger receptors (e.g. scavenger receptor A and CD36). Unlike the LDL receptor, scavenger receptor-mediated uptake of cholesterol is not downregulated upon high LDL concentrations. ${ }^{49,50}$ As a result, excessive scavenger receptor-mediated uptake of these LDL particles transformed KCs into lipid-laden (foamy) cells and increased the hepatic inflammatory response. In line, mice deficient for these scavenger receptors demonstrated a strong reduction in hepatic inflammation. ${ }^{51,52}$ Strikingly, despite the difference in inflammation, KC size remained similar, suggesting that intracellular cholesterol trafficking defects in KCs underlie hepatic inflammation. Further observations from our group demonstrate that hyperlipidemic $\mathrm{Ldlr}^{-/}$mice with hepatic inflammation display increased cholesterol accumulation inside lysosomes of KCs. Rather than lysosomal storage, cholesterol was profoundly stored inside the cytoplasm of KCs of mice without hepatic inflammation (See Figure 1.3). ${ }^{52,53}$ Thus, a strong association exists between increased lysosomal cholesterol accumulation in KCs and hepatic inflammation. ${ }^{54}$ Accordingly, instead of subjects with steatosis, the presence of foamy KCs was exclusively found in liver biopsies of NASH patients and suggests the existence of a similar cholesterol trafficking defect in humans as identified in hyperlipidemic mice. ${ }^{55}$ Taken together, these data suggest that not the foamy appearance of KCs but the intracellular cholesterol distribution plays a crucial role in the initiation of NASH. 

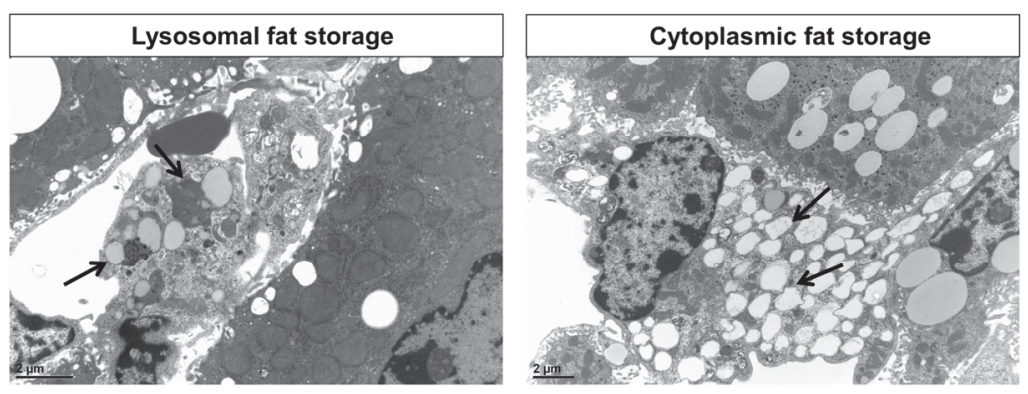

Figure 1.3 Electron microscopy pictures of Kupffer cells of hyperlipidemic LdIr/- mice. Mice with increased hepatic inflammation correlated with more cholesterol storage inside lysosomes (left), as pointed out by the arrows, whereas mice with reduced hepatic inflammation demonstrated cholesterol deposition inside the cytoplasm (right).

Due to the combination of elevated cholesterol-rich LDL particles in plasma and increased oxidative stress, higher oxLDL levels are observed in obese and NASH subjects. ${ }^{56,57}$ Whereas non-modified LDL is efficiently cleared from lysosomes, modified LDL, especially in the form of oxLDL, was resistant to normal efflux and lead to accumulation in the lysosomal compartment. ${ }^{58}$ Additional in vitro research demonstrated that the uptake of oxLDL, and not native LDL, by macrophages is the trigger for the inflammatory response. ${ }^{59-61}$ In line, a mouse model for studying NASH was developed by adding oxLDL to the high-fat diet. ${ }^{62}$ These data suggest that inflammation is dependent on lysosomal cholesterol accumulation of specific lipoprotein species and point towards lysosomal oxLDL accumulation as the main trigger for the inflammatory response. However, rather than being a direct trigger for $\mathrm{NASH}$, these studies all demonstrated an association between oxLDL and inflammation.

Lysosomal cholesterol accumulation leads to an increase in the size of the lysosomes and a lipid-swollen phenotype. Such cholesterol overload inside lysosomes has been associated with lysosomal dysfunction. ${ }^{63}$ Recently, it becomes clear that the function of the lysosome includes more than the digestive system of the cell. Increasing evidence now demonstrates that the lysosomal compartment can also be viewed as vesicles that can secrete its content, for example lysosomal enzymes. ${ }^{64,65}$ In line, uptake of oxLDL has been shown to increase lysosomal destabilization ${ }^{66,67}$ and may induce relocation of lysosomal enzymes, such as cathepsin D. ${ }^{68,69}$ In turn, cathepsin D has been shown to be involved in activation of the inflammasome and apoptosis, thereby potentially leading to inflammation. ${ }^{70}$ So far, little is known about lysosomes in the context of NASH. 


\section{Thesis aim and outline}

Despite the demonstrated strong association between lysosomal cholesterol accumulation and hepatic inflammation, a causal link was never investigated. In the current thesis, we aimed to establish the causality between lysosomal oxLDL accumulation inside KCs and hepatic inflammation. Furthermore, based on our observations regarding the fundamental role of oxLDL in NASH, novel treatment options were tested and an early as well as accurate diagnostic method for the detection of NASH was developed.

Chapter 2 points toward an association between oxLDL and hepatic inflammation and provides a detailed overview about the harmful and inflammatory effects of oxLDL. In Chapter $\mathbf{3}$ we showed the association between lysosomal cholesterol storage and hepatic inflammation. Furthermore, we demonstrated specifically that, unlike native and acetylated LDL, it is the lipoprotein fraction oxLDL that tends to accumulate inside the lysosomes of KCs. In Chapter 4, we demonstrated a direct causal link between lysosomal cholesterol accumulation in $\mathrm{KCs}$ and hepatic inflammation. For this purpose, we transplanted bone marrow of $N p c 1^{\text {mutant }}$ mice into $L d l r^{-/-}$mice as a tool to induce lysosomal cholesterol accumulation inside KCs. Instead of the total cholesterol content inside lysosomes of KCs, we established it is particularly the lysosomal oxLDL that is one of the leading triggers for hepatic inflammation. Chapter $\mathbf{5}$ explores the effect of an oxLDL-targeted immune response during $\mathrm{NASH}$ in $\mathrm{Ld}^{-/-}$mice. The immunization gave rise to specific anti-oxLDL antibodies and was tested as a specific treatment intervention to prevent the development of NASH. In Chapter 6, we tested the plasma lysosomal enzyme, cathepsin $D$, as a novel diagnostic tool to predict hepatic inflammation in children. For this purpose, we studied a pediatric liver biopsyproven cohort of ninety-six children. Chapter 7 explores plasma cathepsin D levels in three adult NASH cohorts and in NASH patients after intervention. In this chapter, the potential clinical value of cathepsin D as a tool to improve NASH diagnosis during progression and regression is evaluated. Chapter $\mathbf{8}$ provides a summary of multiple mechanisms by which lysosomal oxLDL accumulation inside macrophages can affect hepatic inflammation. Finally, the key results of the thesis are discussed in Chapter 9 and placed in the current state of the field. Furthermore, the clinical implications of the findings are addressed. 


\section{References}

1. Wilkin TJ, Voss LD. Metabolic syndrome: maladaptation to a modern world. J R Soc Med 2004;97: 511-20.

2. Zimmet $P, K G$ MMA, Serrano Rios $M$. [A new international diabetes federation worldwide definition of the metabolic syndrome: the rationale and the results]. Rev Esp Cardiol 2005;58:1371-6.

3. Grundy SM. Metabolic syndrome pandemic. Arterioscler Thromb Vasc Biol 2008;28:629-36.

4. Ruderman NB SG. The Metabolic Syndrome. Philadelphia, PA: Elsevier, 2010.

5. Boron WF BE. Chapter 45 - Hepatobiliary Function. In: Medical Physiology - Updated Edition. Philadelphia, PA: Elsevier Saunders, 2005:975-1002.

6. Sheth K, Bankey P. The liver as an immune organ. Curr Opin Crit Care 2001;7:99-104.

7. Anstee QM, McPherson S, Day CP. How big a problem is non-alcoholic fatty liver disease? BMJ 2011; 343:d3897.

8. Bambha K, Belt P, Abraham M, et al. Ethnicity and nonalcoholic fatty liver disease. Hepatology 2012; 55:769-80.

9. Tilg $\mathrm{H}$, Moschen AR. Evolution of inflammation in nonalcoholic fatty liver disease: the multiple parallel hits hypothesis. Hepatology 2010;52:1836-46.

10. Williams CD, Stengel J, Asike MI, et al. Prevalence of nonalcoholic fatty liver disease and nonalcoholic steatohepatitis among a largely middle-aged population utilizing ultrasound and liver biopsy: a prospective study. Gastroenterology 2011;140:124-31.

11. Younossi ZM, Stepanova M, Negro F, et al. Nonalcoholic fatty liver disease in lean individuals in the United States. Medicine (Baltimore) 2012;91:319-27.

12. Daniel S, Ben-Menachem T, Vasudevan G, et al. Prospective evaluation of unexplained chronic liver transaminase abnormalities in asymptomatic and symptomatic patients. Am J Gastroenterol 1999;94: 3010-4.

13. Skelly MM, James PD, Ryder SD. Findings on liver biopsy to investigate abnormal liver function tests in the absence of diagnostic serology. J Hepatol 2001;35:195-9.

14. Machado $M$, Marques-Vidal $\mathrm{P}$, Cortez-Pinto $\mathrm{H}$. Hepatic histology in obese patients undergoing bariatric surgery. J Hepatol 2006;45:600-6.

15. Wanless IR, Lentz JS. Fatty liver hepatitis (steatohepatitis) and obesity: an autopsy study with analysis of risk factors. Hepatology 1990;12:1106-10.

16. Nobili V, Pinzani M. Paediatric non-alcoholic fatty liver disease. Gut 2010;59:561-4.

17. Feldstein $\mathrm{AE}$, Charatcharoenwitthaya $\mathrm{P}$, Treeprasertsuk $\mathrm{S}$, et al. The natural history of non-alcoholic fatty liver disease in children: a follow-up study for up to 20 years. Gut 2009;58:1538-44.

18. Ogden $\mathrm{CL}$, Carroll MD, Kit BK, et al. Prevalence of childhood and adult obesity in the United States, 2011-2012. JAMA 2014;311:806-14.

19. Falck-Ytter $Y$, Younossi ZM, Marchesini G, et al. Clinical features and natural history of nonalcoholic steatosis syndromes. Semin Liver Dis 2001;21:17-26.

20. Kim WR, Flamm SL, Di Bisceglie AM, et al. Serum activity of alanine aminotransferase (ALT) as an indicator of health and disease. Hepatology 2008;47:1363-70.

21. Mofrad $\mathrm{P}$, Contos MJ, Haque M, et al. Clinical and histologic spectrum of nonalcoholic fatty liver disease associated with normal ALT values. Hepatology 2003;37:1286-92.

22. Ochi H, Hirooka M, Koizumi $\mathrm{Y}$, et al. Real-time tissue elastography for evaluation of hepatic fibrosis and portal hypertension in nonalcoholic fatty liver diseases. Hepatology 2012;56:1271-8.

23. Kim DY, Park SH, Lee SS, et al. Contrast-enhanced computed tomography for the diagnosis of fatty liver: prospective study with same-day biopsy used as the reference standard. Eur Radiol 2010;20: 359-66.

24. Noureddin M, Lam J, Peterson MR, et al. Utility of magnetic resonance imaging versus histology for quantifying changes in liver fat in nonalcoholic fatty liver disease trials. Hepatology 2013;58:1930-40.

25. McPherson S, Jonsson JR, Cowin GJ, et al. Magnetic resonance imaging and spectroscopy accurately estimate the severity of steatosis provided the stage of fibrosis is considered. J Hepatol 2009;51: 389-97.

26. Saadeh S, Younossi ZM, Remer EM, et al. The utility of radiological imaging in nonalcoholic fatty liver disease. Gastroenterology 2002;123:745-50. 
27. Kleiner DE, Brunt EM, Van Natta M, et al. Design and validation of a histological scoring system for nonalcoholic fatty liver disease. Hepatology 2005;41:1313-21.

28. Brunt EM, Kleiner DE, Wilson LA, et al. Nonalcoholic fatty liver disease (NAFLD) activity score and the histopathologic diagnosis in NAFLD: distinct clinicopathologic meanings. Hepatology 2011;53:810-20.

29. Alkhouri N, De Vito R, Alisi A, et al. Development and validation of a new histological score for pediatric non-alcoholic fatty liver disease. J Hepatol 2012;57:1312-8.

30. Bravo AA, Sheth SG, Chopra S. Liver biopsy. N Engl J Med 2001;344:495-500.

31. Ratziu V, Charlotte F, Heurtier A, et al. Sampling variability of liver biopsy in nonalcoholic fatty liver disease. Gastroenterology 2005;128:1898-906.

32. Krasnoff JB, Painter PL, Wallace JP, et al. Health-related fitness and physical activity in patients with nonalcoholic fatty liver disease. Hepatology 2008;47:1158-66.

33. Neuschwander-Tetri BA. Lifestyle modification as the primary treatment of NASH. Clin Liver Dis 2009; 13:649-65.

34. Dixon JB, Bhathal PS, O'Brien PE. Nonalcoholic fatty liver disease: predictors of nonalcoholic steatohepatitis and liver fibrosis in the severely obese. Gastroenterology 2001;121:91-100.

35. Satapathy SK, Sanyal AJ. Novel treatment modalities for nonalcoholic steatohepatitis. Trends Endocrinol Metab 2010;21:668-75.

36. Lindor KD, Kowdley KV, Heathcote EJ, et al. Ursodeoxycholic acid for treatment of nonalcoholic steatohepatitis: results of a randomized trial. Hepatology 2004;39:770-8.

37. Adams LA, Zein CO, Angulo P, et al. A pilot trial of pentoxifylline in nonalcoholic steatohepatitis. Am J Gastroenterol 2004;99:2365-8.

38. Lirussi F, Mastropasqua E, Orando S, et al. Probiotics for non-alcoholic fatty liver disease and/or steatohepatitis. Cochrane Database Syst Rev 2007:CD005165.

39. Wong VW, Won GL, Chim AM, et al. Treatment of nonalcoholic steatohepatitis with probiotics. A proof-of-concept study. Ann Hepatol 2013;12:256-62.

40. Bernsmeier C, Meyer-Gerspach AC, Blaser LS, et al. Glucose-induced glucagon-like Peptide 1 secretion is deficient in patients with non-alcoholic fatty liver disease. PLoS One 2014;9:e87488.

41. Blaslov K, Bulum T, Zibar K, et al. Incretin based therapies: a novel treatment approach for nonalcoholic fatty liver disease. World J Gastroenterol 2014;20:7356-65.

42. Neuschwander-Tetri BA, Loomba R, Sanyal AJ, et al. Farnesoid X nuclear receptor ligand obeticholic acid for non-cirrhotic, non-alcoholic steatohepatitis (FLINT): a multicentre, randomised, placebocontrolled trial. Lancet 2015;385:956-65.

43. Anstee QM, Goldin RD. Mouse models in non-alcoholic fatty liver disease and steatohepatitis research. Int J Exp Pathol 2006;87:1-16.

44. Ishibashi S, Brown MS, Goldstein JL, et al. Hypercholesterolemia in low density lipoprotein receptor knockout mice and its reversal by adenovirus-mediated gene delivery. J Clin Invest 1993;92:883-93.

45. Bieghs V, Van Gorp PJ, Wouters K, et al. LDL receptor knock-out mice are a physiological model particularly vulnerable to study the onset of inflammation in non-alcoholic fatty liver disease. PLoS One 2012;7:e30668.

46. Baffy G. Kupffer cells in non-alcoholic fatty liver disease: the emerging view. J Hepatol 2009;51: 212-23.

47. Tosello-Trampont AC, Landes SG, Nguyen V, et al. Kupffer cells trigger nonalcoholic steatohepatitis development in diet-induced mouse model through tumor necrosis factor-alpha production. J Biol Chem 2012;287:40161-72.

48. Lotowska JM, Sobaniec-Lotowska ME, Lebensztejn DM. The role of Kupffer cells in the morphogenesis of nonalcoholic steatohepatitis - ultrastructural findings. The first report in pediatric patients. Scand J Gastroenterol 2013;48:352-7.

49. Brown MS, Goldstein JL. A proteolytic pathway that controls the cholesterol content of membranes, cells, and blood. Proc Natl Acad Sci U S A 1999;96:11041-8.

50. Kunjathoor VV, Febbraio M, Podrez EA, et al. Scavenger receptors class A-I/II and CD36 are the principal receptors responsible for the uptake of modified low density lipoprotein leading to lipid loading in macrophages. J Biol Chem 2002;277:49982-8.

51. Bieghs V, Wouters K, van Gorp PJ, et al. Role of scavenger receptor A and CD36 in diet-induced nonalcoholic steatohepatitis in hyperlipidemic mice. Gastroenterology 2010;138:2477-86, 2486 e1-3. 
52. Bieghs V, Verheyen F, van Gorp PJ, et al. Internalization of modified lipids by CD36 and SR-A leads to hepatic inflammation and lysosomal cholesterol storage in Kupffer cells. PLoS One 2012;7:e34378.

53. Bieghs V, Hendrikx T, van Gorp PJ, et al. The cholesterol derivative 27-hydroxycholesterol reduces steatohepatitis in mice. Gastroenterology 2013;144:167-178 e1.

54. Hendrikx T, Walenbergh SM, Hofker MH, et al. Lysosomal cholesterol accumulation: driver on the road to inflammation during atherosclerosis and non-alcoholic steatohepatitis. Obes Rev 2014;15: 424-33.

55. Ioannou GN, Haigh WG, Thorning D, et al. Hepatic cholesterol crystals and crown-like structures distinguish NASH from simple steatosis. J Lipid Res 2013;54:1326-34.

56. Weinbrenner T, Schroder H, Escurriol V, et al. Circulating oxidized LDL is associated with increased waist circumference independent of body mass index in men and women. Am J Clin Nutr 2006;83:305; quiz 181-2.

57. Clark JM. The epidemiology of nonalcoholic fatty liver disease in adults. J Clin Gastroenterol 2006;40 Suppl 1:S5-10.

58. Jerome WG, Cash C, Webber R, et al. Lysosomal lipid accumulation from oxidized low density lipoprotein is correlated with hypertrophy of the Golgi apparatus and trans-Golgi network. J Lipid Res 1998;39:1362-71.

59. Jerome WG, Cox BE, Griffin EE, et al. Lysosomal cholesterol accumulation inhibits subsequent hydrolysis of lipoprotein cholesteryl ester. Microsc Microanal 2008;14:138-49.

60. Griffin EE, Ullery JC, Cox BE, et al. Aggregated LDL and lipid dispersions induce lysosomal cholesteryl ester accumulation in macrophage foam cells. J Lipid Res 2005;46:2052-60.

61. Groeneweg $M$, Kanters $E$, Vergouwe $M N$, et al. Lipopolysaccharide-induced gene expression in murine macrophages is enhanced by prior exposure to oxLDL. J Lipid Res 2006;47:2259-67.

62. Yimin, Furumaki H, Matsuoka S, et al. A novel murine model for non-alcoholic steatohepatitis developed by combination of a high-fat diet and oxidized low-density lipoprotein. Lab Invest 2012; 92:265-81.

63. Liao G, Yao Y, Liu J, et al. Cholesterol accumulation is associated with lysosomal dysfunction and autophagic stress in Npc1 -/- mouse brain. Am J Pathol 2007;171:962-75.

64. Bordon Y. Immune regulation: Iysosomes at the heart of inflammation. Nat Rev Immunol 2011;11: 502.

65. Samie MA, Xu H. Lysosomal exocytosis and lipid storage disorders. J Lipid Res 2014;55:995-1009.

66. Yuan XM, Li W, Olsson AG, et al. The toxicity to macrophages of oxidized low-density lipoprotein is mediated through lysosomal damage. Atherosclerosis 1997;133:153-61.

67. Sheedy FJ, Grebe A, Rayner KJ, et al. CD36 coordinates NLRP3 inflammasome activation by facilitating intracellular nucleation of soluble ligands into particulate ligands in sterile inflammation. Nat Immunol 2013;14:812-20.

68. Li W, Yuan XM, Olsson AG, et al. Uptake of oxidized LDL by macrophages results in partial lysosomal enzyme inactivation and relocation. Arterioscler Thromb Vasc Biol 1998;18:177-84.

69. Hoppe G, O'Neil J, Hoff HF, et al. Products of lipid peroxidation induce missorting of the principal lysosomal protease in retinal pigment epithelium. Biochim Biophys Acta 2004;1689:33-41.

70. Rintahaka J, Lietzen N, Ohman T, et al. Recognition of cytoplasmic RNA results in cathepsindependent inflammasome activation and apoptosis in human macrophages. J Immunol 2011;186: 3085-92. 


\section{Chapter 2}

Non-alcoholic steatohepatitis: The role of oxidized low-density lipoproteins

SMA Walenbergh, GH Koek, V Bieghs, R Shiri-Sverdlov

J Hepatol 2013;58:801-10 


\section{Abstract}

Non-alcoholic steatohepatitis (NASH) is hallmarked by lipid accumulation in the liver (steatosis) along with inflammation (hepatitis). The transition from simple steatosis towards NASH represents a key step in pathogenesis, as it will set the stage for further severe liver damage. Yet, the pathogenesis behind hepatic inflammation is still poorly understood. It is of relevance to better understand the underlying mechanisms involved in NASH in order to apply new knowledge to potential novel therapeutic approaches. In the current review, we propose oxidized cholesterol as a novel risk factor for NASH. Here, we summarize mouse and human studies that provide possible mechanisms for the involvement of oxidized low-density lipoproteins (oxLDL) in NASH and consequent potential novel diagnostic tools and treatment strategies for hepatic inflammation. 


\section{Introduction}

Non-alcoholic fatty liver disease (NAFLD) involves a cluster of liver disease pathologies ranging from liver lipid accumulation (steatosis) through inflammation (non-alcoholic steatohepatitis) to fibrosis and finally, irreversible cirrhosis. Compared to simple steatosis, non-alcoholic steatohepatitis (NASH) is a more severe, but less common form of NAFLD. According to a prospective study, approximately $46 \%$ of a general patient population was classified with a fatty liver, of which $29 \%$ of ultrasound positive subjects were diagnosed with biopsy-proven NASH. Parallel to the increasing prevalence of obesity, there was a corresponding increase of body mass index (BMI) in this cohort. ${ }^{1}$ Concomitantly, weight loss improved the histological disease activity of $\mathrm{NASH}^{2}{ }^{2}$ Since obesity is a growing international epidemic both in adults and children, steatohepatitis is about to become the most common cause of liver cirrhosis and endstage liver diseases, due to the complications of portal hypertension. ${ }^{3}$

As of today, several mechanisms have been proposed for hepatic inflammation. Current interests implicate an important contribution of the adipose tissue, particularly visceral adipose tissue (VAT) and its secretory products. ${ }^{4}$ Abnormal VAT function, primarily due to obesity, amplifies the release of adipocytokines from fatty tissue, which can lead to systemic effects, such as low-grade systemic inflammation and an altered metabolic state with insulin resistance. The increased lipid content in VAT, enhances free fatty acid (FFA) delivery from the adipocytes into the liver, impairing the hepatic lipid content and initiating hepatic insulin resistance. Whereas adipocytokines, including interleukin- 8 and tumor necrosis factor-alpha (TNF- $\alpha$ ), could contribute to hepatic inflammation via lipid peroxidation and modulating the inflammatory response, FFAs can induce NASH via hepatocyte apoptosis, lipotoxicity and increased production of reactive oxygen species (ROS). ${ }^{5,6}$ Recent evidence points toward another tissue, the gastrointestinal tract, as a source for liver inflammation. Apart from altered gut microbiota during obesity, ${ }^{7}$ studies showed increased intestinal permeability during NASH, which could lead to elevated levels of plasma lipopolysaccharide (LPS). ${ }^{8,9}$ This gut-derived LPS can activate the immune system via pro-inflammatory signaling pathways after binding to toll like receptors (TLRs), as those present on, for example Kupffer cells. ${ }^{10}$ Additionally, vascular abnormalities, as observed in atherosclerosis, have been strongly associated with NASH. ${ }^{11}$ Thus, a potential interplay exists between metabolic tissues and inflammation leading to the development of NASH (Figure 2.1). At molecular level, increased FFA levels, among other factors, can initiate endoplasmic reticulum (ER) stress and mitochondrial dysfunction. Subsequently, this will lead to excess ROS production and the formation of lipotoxic molecules, hereby contributing to the hepatic inflammatory response. ${ }^{12}$ Disturbed autophagic function, as a result from decreased removal of altered mitochondria and the ER, has been suggested to further aggravate hepatic infammation. ${ }^{13}$ Thus, several mechanisms play a role in the transition to NASH. Recently, increasing amounts of data show the involvement of oxLDL in hepatic 
inflammation. While there is no evidence that the contribution of oxLDL to NASH is greater than other known mechanisms, oxLDL is emerging as a new risk factor for hepatic inflammation. Therefore, in this review, we will focus on oxLDL and its implications in NASH.

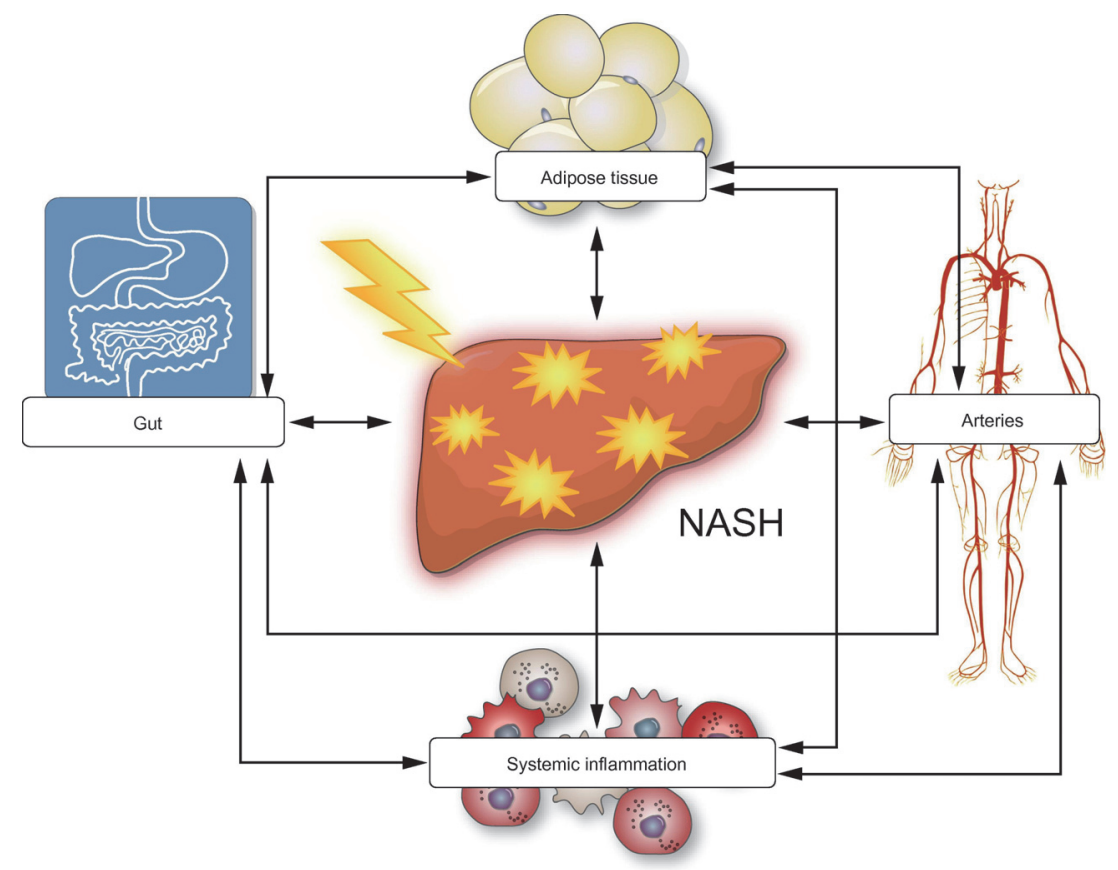

Figure 2.1 Schematic diagram illustrating the metabolic crosstalk between liver, adipose tissue, gut, arteries and systemic inflammation. The development of NASH is dependent on underlying mechanisms related to the metabolic syndrome such as disturbed intestinal permeability, gut microbiota, increased systemic inflammation, vascular abnormalities, and adipose tissue dysfunction as a result from increased macrophage infiltration and insulin resistance. In turn, $\mathrm{NASH}$ by itself can exacerbate inflammation in these metabolic tissues, retaining a positive feedback mechanism.

NAFLD corresponds with an altered lipid metabolism and is associated with the metabolic syndrome (MetS). One central feature is the elevation of triglycerides in plasma as well as in the liver. Sources of increased hepatic triglyceride content are due to excess dietary intake, elevated triglyceride synthesis in the liver from FFA formed during de novo lipogenesis, enhanced FFA influx into the liver from lipolysis of adipose tissue and subsequent conversion into triglycerides, reduced lipid export from the liver via very low-density lipoprotein particles and diminished oxidation of fatty acids. ${ }^{14}$ Other hallmarks associated with NAFLD are low plasma high-density lipoproteins (HDL), elevated low-density lipoproteins (LDL) and total cholesterol. ${ }^{15}$ Currently, it has been postulated that different types of lipids mediate the disease 
spectrum of NAFLD. While hepatic accumulation of triglycerides is related to steatosis, it becomes more evident that cholesterol is implicated in the hepatic inflammatory response. For example, a high cholesterol diet induced liver inflammation in mice susceptible for NASH, while elimination of dietary cholesterol prevented steatohepatitis. ${ }^{16,17}$ Although there is a clear association between obesity and NASH, dietary cholesterol was even found to be the main trigger of hepatic inflammation in non-obese rodents and humans. ${ }^{18,19}$ Moreover, in livers of NASH patients, total plasma cholesterol as well as free cholesterol deposits were found to be increased compared to control subjects. ${ }^{20,21}$ Altogether, these observations indicate that cholesterol is a key player in the onset of NASH.

Oxidative stress is another important and central mechanism in the progression towards NASH. Many cells, including macrophages, are capable of internalizing and accumulating excess amounts of plasma lipoprotein-derived cholesterol. ${ }^{22}$ Mimicking this process in vitro, by loading of macrophages with cholesterol, resulted in increased generation of ROS. ${ }^{23}$ In turn, oxidative stress brings damage to cell structures such as membranes, proteins and DNA of liver cells, hereby triggering a hepatic inflammatory response which can eventually lead to apoptosis. ${ }^{24}$ Several sources of hepatic ROS have been determined regarding the development of NASH and include mitochondria, peroxisomes, the endoplasmic reticulum and enzymes such as the cytochrome P450 superfamily, NAPDH oxidase and xanthine oxidase. ${ }^{24}$ Recently, it has been reported that steatohepatitis may be caused by lipid-induced oxidative stress. ${ }^{25}$ Thus, given that cholesterol and oxidative stress play a causal role in the pathogenesis of NASH, it is highly likely that not cholesterol alone, but consequent oxidation of cholesterol specifically is the substantial risk factor for NASH. To support this hypothesis, we will evaluate current data that describe the involvement of oxidized cholesterol in inflammation and NASH. Additionally, potential clinical benefits of oxLDL in the field of NASH will be discussed.

\section{The inflammatory aspects of oxLDL}

Recent studies show that oxLDL contributes to inflammatory processes through interaction with immune cells and disturbed intracellular cholesterol trafficking. To date, an increasing amount of evidence implicates an important role for oxLDL in obesity-related inflammatory disorders, such as atherosclerosis ${ }^{26,27}$ and cardiovascular disease (CVD). ${ }^{28,29}$

So far, several mechanisms underlying LDL oxidation have been identified in vivo. Hyperglycemia, a pre-diabetic state prior to insulin resistance, has been shown to be strongly associated with oxidation of circulating LDL as glucose decreases the antioxidant characteristics of serum albumin. ${ }^{30,31}$ Chronic hyperglycemia has been implicated in the enhanced formation of advanced glycation end products (AGEs), 
eliciting alterations of the LDL particle. ${ }^{32}$ Interestingly, feeding mice a high-AGE diet caused liver inflammation, suggesting that AGE-induced modified LDL plays an important role in inflammation. ${ }^{33}$ The increase of FFA flux, primarily released from adipose tissue, into the liver is strongly linked to insulin resistance and increased oxidative stress, possibly exacerbating oxidation of LDL. ${ }^{34}$

\section{OxLDL-induced inflammation and apoptosis}

Minimally oxidized forms of LDL contain lipid oxidation products without extensive protein modification. Since oxLDL particles stay longer in the plasma, they are more prone for further oxidation. As modification proceeds, the highly oxidized LDL particle turns into a structure similar to pathogen-related epitopes and therefore will be removed from plasma through binding and uptake by macrophages. This response is initially intended to be protective, however, an excessive amount of lipids will build up inside macrophages, leading to a phenomenon called foam cell formation. ${ }^{35}$ This change in foamy appearance causes the swollen phenotype of the macrophage to activate the transcription factor nuclear factor-kappaB (NF-kB), ${ }^{36}$ hereby inducing the production of inflammatory cytokines (Figure 2.2). ${ }^{36,37}$ OxLDL has been shown to modulate inflammation by affecting several other cellular mechanisms, such as; inducing transmigration of neutrophils, ${ }^{38}$ eosinophils, ${ }^{39}$ monocytes and Tlymphocytes; ${ }^{40}$ elevating several adhesion molecules ${ }^{38,41-43}$ and recruiting immune cells through the release of the chemokine (C-C motif) ligand 23 (CCL23). ${ }^{44}$ Moreover, oxLDL induced inflammation through increased ROS generation ${ }^{45}$ and elevated expression of metalloproteinases. ${ }^{46}$

Another important aspect during the pathogenesis of inflammation is apoptotic cell death, which has been shown to play an important role in NASH. ${ }^{47,48}$ OxLDL has been found to increase apoptosis through activation of apoptotic signaling cascades including the Fas signaling pathway. ${ }^{49}$ Additionally, biologically active oxidized lipids were found in apoptotic cells. ${ }^{50}$ Thus, given that oxLDL induces apoptosis, oxLDL is not merely an inflammatory trigger, but also promotes subsequent cell damage.

\section{Disturbed intracellular trafficking of oxLDL}

OxLDL possibly exerts its inflammatory effects upon receptor-mediated macrophage endocytosis. Once internalized, it has been postulated that oxLDL is transported to the lysosomal compartment where it is poorly degraded or hydrolyzed and therefore accumulates in lysosomes. This is in contrast to native or acetylated LDL (acLDL), which are normally degraded by lysosomal enzymes followed by relocation into the cytoplasm for further processing. ${ }^{51}$ Lysosomal trapping of oxLDL, probably due to impaired cholesteryl ester hydrolysis or an alteration in lysosomal $\mathrm{pH}^{52}$ has the potential to damage and disrupt the lysosomal membrane. Since lysosomes are involved in a wide variety of biological processes, cholesterol-induced lysosomal 
damage can lead to inflammation and apoptosis. ${ }^{53}$ In vitro data demonstrated the appearance of cholesterol crystals inside lysosomes upon prolonged oxLDL incubation. It is speculated that these crystals represent an endogenous danger signal and trigger the activation of the NLRP3 inflammasome and subsequent pro-inflammatory

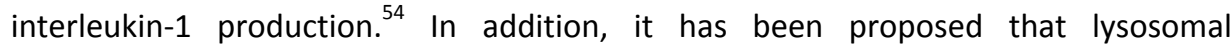
cholesterol accumulation leads to disturbed autophagy, a process important in inflammation and apoptosis. ${ }^{55}$ Taken together, lysosomal trapping of oxLDL inside macrophages leads to cellular damage, possibly through mediating inflammation and apoptosis. Although the inflammatory effects of oxLDL are well-documented in the atherosclerosis field, the link between NASH and CVD has never been investigated directly by any study. Therefore, it is still questionable whether the inflammatory aspects of oxLDL summarized in this paragraph can be applied to NASH as well.

\section{OxLDL and its implications in non-alcoholic steatohepatitis}

An increasing amount of studies strongly emphasize the role of Kupffer cells (KCs), the liver's resident macrophage population, in the pathogenesis of $\mathrm{NASH} .{ }^{56}$ We now elucidate the effect of oxLDL on KCs and its contribution to hepatic inflammation.

\section{Role of KCs}

A growing body of evidence contradicts the "two-hit" model, in which is described that hepatic steatosis is considered to be the first critical 'hit' and a necessary prerequisite for further liver damage, such as inflammation. ${ }^{57}$ Nowadays, it becomes increasingly clear that a multifactor etiology, with a central role for $K C s$, underlies the pathogenesis of NASH. ${ }^{58}$ In contrast to the "two-hit" model, several papers describe the development of severe hepatic inflammation without the presence of hepatic steatosis. ${ }^{16,59}$ Furthermore, omitting cholesterol from hyperlipidemic mice prevented hepatic inflammation without affecting steatosis. ${ }^{16}$ Furthermore, comparable to foam cell formation in atherosclerosis, hyperlipidemic mice showed bloated foamy KCs which was correlated to hepatic inflammation. Consistently, a high fat diet (HFD) without added cholesterol demonstrated reduced hepatic inflammation without swollen KCs. ${ }^{16}$ During early steatohepatitis, isolated fat-laden KCs from HFD-fed mice predominantly contained cholesterol and displayed a pro-inflammatory phenotype. ${ }^{60}$ Inflammation, triggered by cholesterol-rich foam cells, is a well established hypothesis in the field of CVD and has been recognized as a significant parameter during atherosclerotic plaque formation. ${ }^{61}$ Thus, cholesterol or its modified form, trapped inside $\mathrm{KCs}$, is an actual trigger for $\mathrm{NASH}$.

Critical contributors for the uptake of modified lipids and cholesterol by macrophages are the scavenger receptors (SRs), scavenger receptor A (SR-A) and CD36. ${ }^{62}$ Literature 
describes a distinct affinity for binding of oxLDL between these two SRs. SR-A binds and mediates uptake of oxLDL to a lesser extent than CD36. Compared to incubation with $L D L$ and acLDL, treatment with oxLDL elevated gene expression and protein levels of SR-A and CD36 in macrophages. ${ }^{63,64}$ These data show that both SRs are involved in the uptake of oxLDL. Similar to typical macrophages, SRs were also identified on KCs. ${ }^{65}$ Haematopoietic deletion of SR-A (Msr1) and/or Cd36 in hyperlipidemic mice resulted in decreased hepatic inflammation, indicating that SRmediated uptake of modified cholesterol by KCs is the trigger for the development of steatohepatitis (Figure 2.2). ${ }^{59,66}$ Loading bone marrow-derived macrophages of LDL receptor $(L d l r)^{-1-}$ mice with oxLDL, hereby mimicking foam cell formation, showed to be more inflammatory than macrophages without oxLDL loading. ${ }^{67}$ Taken together, these data demonstrate the causal role of oxLDL as a driver of the inflammatory response.
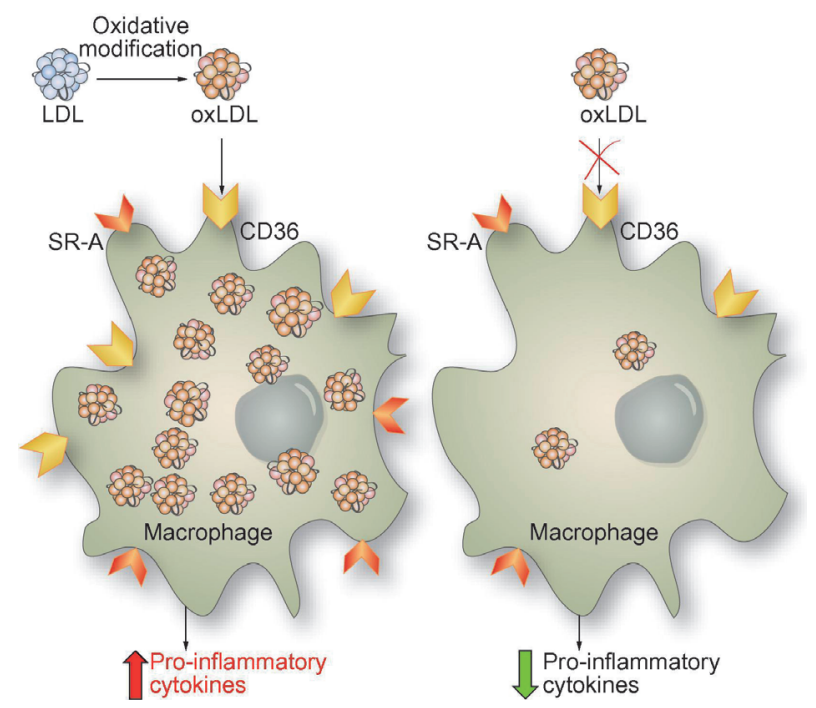

Figure 2.2 Schematic illustration showing the involvement of oxLDL in the macrophage inflammatory response. Lipid-laden foamy macrophages express higher levels of the scavenger receptors CD36 and SR-A and produce more pro-inflammatory cytokines. Through interplay with surrounding cells, these cytokines further amplificate the inflammatory response. Blocking macrophage uptake of oxLDL led to macrophages smaller in size, less CD36 and SR-A expression and reduced inflammation. Modified after ${ }^{135}$.

Recently, a novel mouse model for NASH has been developed by using a combination of oxLDL and a HFD. Administration of oxLDL to wildtype HFD-fed mice displayed the entire pathology of NASH, i.e. steatosis, hepatic inflammation, fibrosis, and also lipidladen macrophages, dyslipidemia and aggravated hepatic lipid peroxidation. ${ }^{64}$ This novel animal model shows the direct involvement of oxLDL in the development of 
$\mathrm{NASH}$, however, the underlying intracellular pathway that contributes to hepatic inflammation has not been established. One proposed theory is a defective intrinsic mechanism of lipid trafficking inside KCs.

Macrophage-derived foam cells, as those present during atherosclerosis, predominantly contain enlarged lysosomes filled with cholesterol and cholesterol crystals, instead of cholesterol ester storage into the cytoplasm. ${ }^{54,68}$ For the first time, our group demonstrated accumulation of cholesterol and cholesterol crystals inside lysosomes of KCs in a mouse model representing NASH. ${ }^{66,69}$ In line with these data, hepatic inflammation was found to be associated with increased cholesterol storage inside lysosomes of $\mathrm{KCs}$, providing evidence that lysosomal cholesterol accumulation in $\mathrm{KCS}$ is crucial for inflammation in the context of NASH. ${ }^{66,69}$ Altogether, mounting evidence demonstrate that NASH exhibits similar characteristics to atherosclerosis, including foam cell formation and cholesterol-engorged lysosomes. Regarding the latter observation, it has been proposed that advanced stages of atherosclerosis are

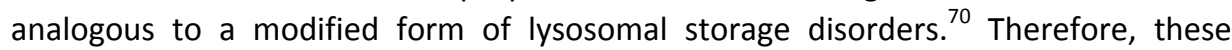
results indicate that NASH can be considered likewise. Our novel hypothesis that NASH shares similarities with an acquired lysosomal storage disorder opens up entirely new therapy possibilities for hepatic inflammation.

By interfering with the immune response, more evidence was provided for the relevant role of oxLDL in NASH. Oxidation structurally modifies the LDL particle, whereby the phosphorylcholine (PC) headgroup, one of the so-called oxidationspecific epitopes, can be found on the outer surface. ${ }^{71}$ Oxidation-specific epitopes are viewed as damage associated molecular patterns (DAMPs) and therefore serve as ligands for immune recognition. ${ }^{72}$ Since these PC epitopes are also present on the capsular polysaccharide cell wall of Streptococcus pneumoniae, ${ }^{73}$ cross reactivity exists between PC epitopes from oxLDL and this bacterium. Therefore, a protective effect against NASH upon active immunization with heat-inactivated S. pneumoniae in $\mathrm{Ldlr}^{-/-}$ mice was found. Immunized mice fed a high fat cholesterol diet showed less foamy KCs, decreased hepatic inflammation and reduced cholesterol crystals inside lysosomes of KCs compared to mice without immunization. ${ }^{69}$ More importantly, reduced inflammation was associated with lower cholesterol oxidation and an increase of IgM autoantibody levels against modified LDL in plasma. ${ }^{59}$ These data strongly suggest that anti-oxLDL antibodies of the IgM subtype are protective against steatohepatitis (Figure 2.2), supporting our hypothesis that oxLDL plays an important role in the development of NASH.

\section{Crosstalk between $\mathrm{KCs}$ and other cell types in the liver}

Activation of $\mathrm{KCs}$ leads to a rapid release of a wide range of inflammatory mediators and signaling molecules such as cytokines, ROS, proteases and lipid mediators. ${ }^{74}$ One of the stimuli that has been shown to activate macrophages and to increase pro- 
inflammatory cytokines, is oxLDL. ${ }^{67}$ Other than oxLDL, different stimuli can activate $\mathrm{KCs}$, such as gut-derived endotoxins ${ }^{75}$ and damaged hepatocytes. For example, due to intercellular communication between hepatocytes and KCs, hepatocyte stress and/or injury results in the excretion of inflammatory mediators, which in turn activate KCs hereby possibly inducing hepatic inflammation. ${ }^{76,77}$ Furthermore, a more advanced stage, the engulfment of apoptotic hepatocytes by KCs promotes their activation and could further contribute to hepatic inflammation. ${ }^{78}$

As discussed earlier, oxLDL trapping inside lysosomes triggers inflammation, most likely due to its activation of KCs.

Once inflammation is elicited, KCs can further spread hepatic injury by amplification of the inflammatory response through interactions with neighbouring hepatocytes, sinusoidial endothelial cells (SECs) and hepatic stellate cells. ${ }^{77}$ Upon activation, KCs primarily release TNF- $\alpha$ and interleukins, ${ }^{79}$ hereby influencing hepatocyte function and viability or indirectly by activating other cells, including SECs. Activation of SECS can indirectly lead to neutrophil-mediated damage to the hepatocytes or even cell death. ${ }^{74}$ Additionally, inflammatory signaling initiated by KCs can be further amplified by the secretion of chemokines, followed by recruitment of infiltrating macrophages and neutrophils. ${ }^{80}$ KC-derived TNF- $\alpha$ contributes to elevated secretion of the chemokine macrophage inflammatory protein 2 (MIP-2) and monocyte chemotactic protein 1 (MCP-1), facilitating activation and infiltration of neutrophils and macrophages into the liver. ${ }^{74,81}$ The hepatic accumulation of neutrophils in turn can lead to hepatotoxicity.

In short, oxLDL is a harmful lipid that causes cellular injury and activation of macrophages and endothelial cells particularly. OxLDL-induced KC activation enhances cytokine-driven hepatocellular signaling pathways, hereby rendering $\mathrm{KCs}$ to further augment inflammation through interaction with other cell types in the liver.

\section{Oxidative stress}

Oxidative stress, the primary risk factor for LDL oxidation, is believed to be a central mechanism in the pathogenesis of NASH. Therefore, in mouse models as well as in human studies, markers for oxidative stress were measured as potential surrogate markers for NASH.

Neutrophils are a potent source of the oxidant-generating enzyme myeloperoxidase (MPO) and are abundantly present in the liver. ${ }^{82,83}$ In vitro data demonstrated that uptake of MPO-induced oxidation of LDL leads to foam cell formation. ${ }^{84}$ In line with this finding, Rensen et al. detected increased MPO-positive KCs in the livers of obese NASH patients, which was accompanied by elevated plasma MPO levels. ${ }^{85}$

During oxidative modification of $L D L$, a diversity of reactive aldehydes on apoB lysine residues are generated by decomposition of lipid peroxidation products, such as 4-hydroxynonenal (HNE) and malondialdehyde (MDA). ${ }^{35}$ Whereas HNE has shown to 
contribute to foam cell formation, MDA modification of lysine residues contributes to functional properties of oxLDL. ${ }^{86}$ Consistently, increased hepatic MDA and HNE levels in rodent models for NASH were identified. ${ }^{47,87}$ Other pivotal contributors to oxidative stress are microsomal cytochrome P450 enzymes, such as P450 2E1, which are mainly located in the liver. Deletion of P450 CYP2E1 in mice resulted in less susceptibility for $\mathrm{NASH}$, decreased oxidized proteins as well as MDA and HNE levels, and protection against insulin resistance compared to their wildtype littermates. ${ }^{88}$ While mouse studies show straightforward results about the role of oxidative stress in NASH, less outspoken data are represented by human studies. Koruk et al. demonstrated an increase of serum MDA in patients with biopsy-proven NASH, while the antioxidants glutathione peroxidase and glutathione reductase showed no difference compared to the control group. ${ }^{89}$ Moreover, an increase of serum thioredoxin, thiobarbituric acid reactive substances (TBARS) and plasma oxLDL was detected in NASH patients in comparison to control subjects. ${ }^{90,91}$ Although the data was statistically significant, a small sample size was used and there were large standard deviations between the groups, considering these studies as being underpowered. Additionally, cohorts were poorly controlled regarding overlapping risk factors for NASH, such as the MetS and/or diabetes. Yet, although a small cohort was used, increased hepatic CYP2E1 activity in non-diabetic NASH patients was demonstrated compared to BMI-matched controls. ${ }^{92}$ Evidence implicates other pro-oxidant enzymes, such as 15 -lipoxygenase and ceruloplasmin, to be involved in the oxidation of LDL. ${ }^{93,94}$ Therefore, clinical data showed in parallel to the progression of NASH, a concomitant increase of enzymatic sources for ROS during hepatic inflammation. ${ }^{15,95}$ Of note, the changes observed in ceruloplasmin levels and P450 liver enzymes are not related to NASH specifically, but also to other aspects of the MetS, including obesity and diabetes mellitus.

In general, most of the human studies presented in this paragraph do not show a causal link between oxidative stress and NASH.

\section{Anti-oxidants}

Oxidative stress represents an oxidant/anti-oxidant imbalance, which is shifted towards greater oxidant activity and/or decreased anti-oxidant levels. Enzymatic and non-enzymatic anti-oxidant defense mechanisms play a role in protecting lipids, such as LDL from oxidation. Observations described above clearly indicate a pro-oxidant state for NASH patients, suggesting there is a diminished anti-oxidant defense status in this population. Indeed, a diversity of anti-oxidant enzymes was found to be reduced in the plasma of NASH patients. ${ }^{89,96}$ Moreover, total anti-oxidant capacity and anti-oxidant enzymes were specifically decreased in the livers of patients with steatohepatitis compared to healthy controls. ${ }^{97}$ Consistent with a decreased activity of anti-oxidant enzymes, non-enzymatic anti-oxidants such as glutathione content and vitamin $\mathrm{E}$ were also diminished in NASH subjects. ${ }^{97,98}$ In parallel with the disease progression of human NAFLD, a decline of glutathione transferase enzyme activity was 
detected in liver specimens. ${ }^{99}$ In striking contrast, extreme low anti-oxidant levels alleviated the progression towards NASH, as observed in glutathione-deficient mice, implicating the activation of a protective compensatory mechanism under severe low anti-oxidant conditions. ${ }^{100}$ Altogether, NASH patients reflect a pro-oxidant state and a reduced anti-oxidant capacity, implying limited ability to counteract oxidation. Thus, these data point towards an important role for oxidation, most likely of LDL, in the development of NASH. However, the decreased level of anti-oxidants as observed in NASH subjects could also be a consequence of other related disorders or risk factors, such as the MetS, obesity and diabetes mellitus.

\section{Clinical implications}

At present, the most accurate diagnostic tool to determine NASH is the histological assessment of a liver biopsy. Due to its invasive procedure, patients experience discomfort and there is a risk for complications $(0.06-0.32 \%)$, including pain, hemorrhage, bile peritonitis and pneumothorax. ${ }^{101}$ The existing non-invasive biomarkers for NASH used in the clinic, i.e. transaminases (ALT, AST), alkaline phosphatase (ALP) and gamma-glutamyl-transpeptidase (GGT) lack specificity and sensitivity to distinguish NASH from steatosis and have been reported as unreliable. ${ }^{102}$ Instead of inflammation, these plasma liver enzymes represent liver damage, of which a novel potential biomarker, plasma cytokeratin 18 , is a marker specifically for hepatocyte apoptosis. ${ }^{103}$ Concerning therapeutics against NASH, there is no proven effective treatment available that specifically reduces hepatic inflammation. Although not all patients fit the following description, NASH patients typically meet the criteria for the MetS, i.e. being obese, insulin resistant and hyperlipidemic. ${ }^{104}$ Therefore, the most adequate recommendation for reducing hepatic inflammation focuses on lifestyle alterations, such as changing nutritional habits and increasing physical activity. ${ }^{104}$ Additional to lifestyle modifications, pharmacological interventions against NASH target hyperlipidemia, insulin resistance and oxidative stress and are therefore similar to that of the MetS. Altogether, non-invasive tests are warranted to diagnose NASH at early stages of the disease process, to allow opportunities to prevent further progression towards severe and irreversible liver damage, such as fibrosis and cirrhosis. Moreover, there is a need for novel and safe therapeutic strategies against $\mathrm{NASH}$ that lead to a pronounced reduction in hepatic inflammation.

\section{Plasma oxLDL}

Higher circulating oxLDL levels were detected in CVD patients compared to healthy subjects. ${ }^{105}$ Generally, the important role of plasma oxLDL has been reviewed extensively for atherosclerosis. ${ }^{26,35}$ In line, Binder et al. have shown to reduce atherosclerosis by inducing protective plasma anti-oxLDL antibodies in mice. ${ }^{106}$ 
Similarly, we have recently shown that these antibodies are also effective against NASH. ${ }^{69}$ Thus, these data point towards oxLDL as a potential target for the prevention of both atherosclerosis and NASH. However, clinical studies are at their infancy and comparative studies of testing various assays to monitor oxLDL are needed to assess which assays have enhanced clinicaly utility for detecting CVD and NASH. So far, none of the tested assays are approved for routine clinical use. ${ }^{29}$

As for diagnosis, oxLDL is not used as a marker to detect atherosclerosis. Similarly, while we found an association between antibodies against oxLDL and NASH, there is no sufficient evidence to suggest that plasma oxLDL can be used as a non-invasive marker to detect hepatic inflammation. To evaluate the prognostic value of plasma oxLDL for the detection of NASH, several bigger cohort studies are necessary.

\section{Anti-oxLDL antibodies}

The finding that oxidation-specific epitopes are not merely present on oxLDL, but also on apoptotic cells, ${ }^{107}$ reflects the link between oxLDL and tissue damage. Therefore, anti-oxLDL antibodies have been shown to be predictors of inflammatory diseases, such as atherosclerosis and CVD. ${ }^{108,109}$ In line with these findings, we have found that plasma IgM anti-oxLDL antibodies correlate negatively with hepatic inflammation in mice. ${ }^{69}$ In this view, anti-oxLDL antibodies can potentially be used as a diagnostic tool for the detection of NASH. However, it is important to note that the amount of antioxLDL antibodies may differ naturally between people and can vary over time. ${ }^{110,111}$ Additionally, molecular mimicry exists between oxidation-specific epitopes of oxLDL and epitopes located on infectious agents, suggesting that exposure to pathogens influences the production of anti-oxLDL antibodies. ${ }^{110}$ This argument may not be beneficial for the use of anti-oxLDL antibodies for the diagnosis of NASH, yet it opens up promising therapeutic strategies against liver inflammation. Boosting the production of anti-oxLDL antibodies via immunization approaches ameliorated atherosclerosis. ${ }^{106,112}$ Since atherosclerosis shares features with NASH, i.e. foam cell formation and inflammation, these immunization approaches hold promise as a treatment against NASH and should be tested clinically in the future.

\section{Cholesterol lowering medication}

NASH patients commonly represent hypertriglyceridemia and hypercholesterolemia, suggesting that NASH is strongly associated with hyperlipidemia. ${ }^{113}$ Therefore, lipidlowering agents, such as polyunsaturated fatty acids (PUFAs), fibrates and statins have been tested in patients with NASH. Recent work reported a positive effect of PUFAs on lobular inflammation and ballooning of the liver in mice, as well as in human NASH, although the human study lacked a control group. ${ }^{114,115}$ Therefore, it has been proposed that randomized controlled trials of adequate size are needed in the future to propose such PUFA treatment to NASH patients. ${ }^{2}$ 
The use of fibrates, which are ligands for the peroxisome proliferator-activated receptor, and statins are still controversial. Fenofibrate administered to mice has been shown to ameliorate hepatic inflammation, while human studies demonstrated no difference in plasma liver enzymes or without changes in histological endpoints for NASH. ${ }^{116-118}$ Statin therapy was investigated by human pilot studies, but only in a limited number of patients. ${ }^{119-121}$ Short-term outcomes show promising results on liver inflammation, as proven by serum aminotransferase activities and liver histology. ${ }^{119-121}$ In addition to their anti-inflammatory properties, statins are generally targeted at lowering lipids. Interestingly, patients who received statins even demonstrated reduced oxLDL, which could be relevant for NASH patients with increased plasma oxLDL levels. ${ }^{122}$ Still, statin-treated NAFLD patients developed advanced fibrosis based on liver histology after a long-term follow-up period. ${ }^{119,123}$ In conclusion, the beneficial effects of statins and fibrates on NASH are still debatable, due to clear limitations to monitor NASH. While some human studies use unspecific plasma liver enzymes, other studies assess liver histology for the development of $\mathrm{NASH}$. Furthermore, there is a clear lack in repeated measurements to monitor NASH progression. Moreover, the difference in beneficial outcome after statin therapy could be explained by the fact that statins are directed at lipid lowering in general and are not directly related to oxLDL. Therefore, future adequate and well-designed human intervention studies examining the effect of statins or fibrates on NAFLD/NASH should be conducted. To monitor long-term statin or fibrate therapy on the development of NASH in human studies, liver histology assessment is critical.

\section{Anti-oxidant therapy}

A pivotal contributor to the pathophysiology of NASH includes oxidative stress. As prooxidant activity is paralleled with oxidation of lipids, including LDL, anti-oxidants have the potential to treat NASH. Promising results were obtained during a clinical trial where non-diabetic NASH patients were randomly assigned to receive the antioxidant, vitamin E, or placebo for 96 weeks. Vitamin $\mathrm{E}$ treatment improved individual features of $\mathrm{NASH}$, such as lobular inflammation and hepatocellular ballooning, as well as the overall NAFLD activity score. ${ }^{124} A$ similar positive outcome on the NASH phenotype was demonstrated in a clinical trial where NASH patients received the antioxidant pentoxifylline. ${ }^{125}$ Vitamin $\mathrm{E}$ has been shown to inhibit CD36-mediated uptake of oxLDL, hereby preventing foam cell formation, whereas pentoxifylline reduced oxLDL-induced leukocyte adhesion to the endothelium and downregulated the integrin receptor $\mathrm{CD} 11 \mathrm{~b} / \mathrm{CD} 18 .^{43,126}$ Additional clinical studies could not attribute a favorable effect to vitamin $E$ and pentoxifylline treatment in the development of NASH. ${ }^{127,128}$ Nevertheless, this could be due to the variable disease course of NAFLD/NASH, sampling error during liver biopsy ${ }^{129}$ and the use of plasma transaminases as a non-specific predictor for NASH. ${ }^{102}$ Although further investigations are needed, other anti-oxidants have also shown to be effective against NASH and 
include ursodeoxycholic acid with or without vitamin $E_{,}^{130,131}$ betaine and other dietary supplements. ${ }^{87,132-134}$ In summary, anti-oxidant therapy, either via supplementation of anti-oxidants or agents that increase the generation of antioxidant enzymes, seems to be effective in reducing NASH. Even though anti-oxidant therapy counteracts oxidative stress and thereby inflammation, anti-oxidants might serve as a useful adjunct therapy to support targeted therapies.

\section{Concluding remarks}

A number of studies demonstrated a close relationship between the MetS and increased plasma oxLDL levels. In recent years, a greater amount of evidence therefore linked oxLDL with the pathogenesis of $\mathrm{NASH}$, the hepatic manifestation of the MetS. Already for a long time, it has been known that oxLDL is cytotoxic and induces cellular damage. However, until recently, oxLDL has also been found to exert its harmful effects on KCs, followed by KC-derived interplay with other hepatic cells. The reviewed data suggest for the first time that oxLDL is an important trigger for NASH development. Since cholesterol and its oxidized form play a crucial role in the progression of NAFLD, most therapeutic strategies against NASH should aim at lowering plasma cholesterol, prevention of (oxidized) cholesterol uptake by macrophages and enhancement of the whole-body anti-oxidant status. The finding that NASH can be viewed as an acquired lysosomal storage disorder has significant implications for the development of novel therapeutics against liver inflammation. Higher oxLDL levels in the plasma do not necessarily discriminate NASH from its overlapping risk factors, obesity, diabetes or atherosclerosis. On the one hand, lowering plasma oxLDL has therefore additional beneficial effects on metabolic related disorders. On the other hand such an overlap puts the diagnostic value of plasma oxLDL, and its specificity to detect NASH, at risk. Therefore, we suggest that studies in mice and large human cohorts should be used in the future to test the clinical utility of plasma oxLDL as a non-invasive marker for NASH. All in all, these diagnostic and therapeutic strategies provide a basis for the amelioration of NASH and related metabolic risk factors that can lead to CVD, diabetes mellitus and its associated complications. 


\section{References}

1. Williams CD, Stengel J, Asike MI, Torres DM, Shaw J, Contreras M, et al. Prevalence of nonalcoholic fatty liver disease and nonalcoholic steatohepatitis among a largely middle-aged population utilizing ultrasound and liver biopsy: a prospective study. Gastroenterology 2011;140:124-31.

2. Musso G, Gambino R, Cassader M, Pagano G. A meta-analysis of randomized trials for the treatment of nonalcoholic fatty liver disease. Hepatology 2010;52:79-104.

3. lacobellis A, Marcellini M, Andriulli A, Perri F, Leandro G, Devito R, et al. Non invasive evaluation of liver fibrosis in paediatric patients with nonalcoholic steatohepatitis. World J Gastroenterol 2006;12:7821-5.

4. Fain JN, Madan AK, Hiler ML, Cheema P, Bahouth SW. Comparison of the release of adipokines by adipose tissue, adipose tissue matrix, and adipocytes from visceral and subcutaneous abdominal adipose tissues of obese humans. Endocrinology 2004;145:2273-82.

5. Schaffler A, Scholmerich J, Buchler C. Mechanisms of disease: adipocytokines and visceral adipose tissue--emerging role in nonalcoholic fatty liver disease. Nat Clin Pract Gastroenterol Hepatol 2005;2:273-80.

6. Qureshi K, Abrams GA. Metabolic liver disease of obesity and role of adipose tissue in the pathogenesis of nonalcoholic fatty liver disease. World J Gastroenterol 2007;13:3540-53.

7. Cani PD, Possemiers S, Van de Wiele T, Guiot Y, Everard A, Rottier O, et al. Changes in gut microbiota control inflammation in obese mice through a mechanism involving GLP-2-driven improvement of gut permeability. Gut 2009;58:1091-103.

8. Wigg AJ, Roberts-Thomson IC, Dymock RB, McCarthy PJ, Grose RH, Cummins AG. The role of small intestinal bacterial overgrowth, intestinal permeability, endotoxaemia, and tumour necrosis factor alpha in the pathogenesis of non-alcoholic steatohepatitis. Gut 2001;48:206-11.

9. Brun P, Castagliuolo I, Di Leo V, Buda A, Pinzani M, Palu G, et al. Increased intestinal permeability in obese mice: new evidence in the pathogenesis of nonalcoholic steatohepatitis. Am J Physiol Gastrointest Liver Physiol 2007;292:G518-25.

10. Ye D, Li FY, Lam KS, Li H, Jia W, Wang Y, et al. Toll-like receptor-4 mediates obesity-induced nonalcoholic steatohepatitis through activation of X-box binding protein-1 in mice. Gut 2012;61:1058-67.

11. Targher G, Bertolini L, Padovani R, Rodella S, Arcaro G, Day C. Differences and similarities in early atherosclerosis between patients with non-alcoholic steatohepatitis and chronic hepatitis B and C. J Hepatol 2007;46:1126-32.

12. Alkhouri N, Dixon $\amalg$, Feldstein AE. Lipotoxicity in nonalcoholic fatty liver disease: not all lipids are created equal. Expert Rev Gastroenterol Hepatol 2009;3:445-51.

13. Amir M, Czaja MJ. Autophagy in nonalcoholic steatohepatitis. Expert Rev Gastroenterol Hepatol 2011;5:159-66.

14. Cheung O, Sanyal AJ. Abnormalities of lipid metabolism in nonalcoholic fatty liver disease. Semin Liver Dis 2008;28:351-9.

15. Koruk M, Savas MC, Yilmaz O, Taysi S, Karakok M, Gundogdu C, et al. Serum lipids, lipoproteins and apolipoproteins levels in patients with nonalcoholic steatohepatitis. J Clin Gastroenterol 2003;37: 177-82.

16. Wouters K, van Gorp PJ, Bieghs V, Gijbels MJ, Duimel H, Lutjohann D, et al. Dietary cholesterol, rather than liver steatosis, leads to hepatic inflammation in hyperlipidemic mouse models of nonalcoholic steatohepatitis. Hepatology 2008;48:474-86.

17. Van Rooyen DM, Larter CZ, Haigh WG, Yeh MM, loannou G, Kuver R, et al. Hepatic free cholesterol accumulates in obese, diabetic mice and causes nonalcoholic steatohepatitis. Gastroenterology 2011;141:1393-1403, 1403 e1391-5.

18. Musso G, Gambino R, De Michieli F, Cassader M, Rizzetto M, Durazzo M, et al. Dietary habits and their relations to insulin resistance and postprandial lipemia in nonalcoholic steatohepatitis. Hepatology 2003;37:909-16.

19. Yasutake K, Nakamuta M, Shima Y, Ohyama A, Masuda K, Haruta N, et al. Nutritional investigation of non-obese patients with non-alcoholic fatty liver disease: the significance of dietary cholesterol. Scand J Gastroenterol 2009;44:471-7. 
20. Caballero F, Fernandez A, De Lacy AM, Fernandez-Checa JC, Caballeria J, Garcia-Ruiz C. Enhanced free cholesterol, SREBP-2 and StAR expression in human NASH. J Hepatol 2009;50:789-96.

21. Puri P, Baillie RA, Wiest MM, Mirshahi F, Choudhury J, Cheung O, et al. A lipidomic analysis of nonalcoholic fatty liver disease. Hepatology 2007;46:1081-90.

22. Tabas I. Consequences of cellular cholesterol accumulation: basic concepts and physiological implications. J Clin Invest 2002;110:905-11.

23. Hung YC, Hong MY, Huang GS. Cholesterol loading augments oxidative stress in macrophages. FEBS Lett 2006;580:849-61.

24. Koek GH, Liedorp PR, Bast A. The role of oxidative stress in non-alcoholic steatohepatitis. Clin Chim Acta 2011;412:1297-305.

25. Matsuzawa N, Takamura T, Kurita S, Misu H, Ota T, Ando H, et al. Lipid-induced oxidative stress causes steatohepatitis in mice fed an atherogenic diet. Hepatology 2007;46:1392-403.

26. Li D, Mehta JL. Oxidized LDL, a critical factor in atherogenesis. Cardiovasc Res 2005;68:353-4.

27. Nishi K, Itabe H, Uno M, Kitazato KT, Horiguchi H, Shinno K, et al. Oxidized LDL in carotid plaques and plasma associates with plaque instability. Arterioscler Thromb Vasc Biol 2002;22:1649-54.

28. Holvoet $P$, Mertens A, Verhamme P, Bogaerts K, Beyens G, Verhaeghe R, et al. Circulating oxidized LDL is a useful marker for identifying patients with coronary artery disease. Arterioscler Thromb Vasc Biol 2001;21:844-8.

29. Fraley AE, Tsimikas S. Clinical applications of circulating oxidized low-density lipoprotein biomarkers in cardiovascular disease. Curr Opin Lipidol 2006;17:502-9.

30. Kopprasch S, Pietzsch J, Kuhlisch E, Fuecker K, Temelkova-Kurktschiev T, Hanefeld M, et al. In vivo evidence for increased oxidation of circulating $\mathrm{LDL}$ in impaired glucose tolerance. Diabetes 2002;51:3102-6.

31. Bourdon E, Loreau N, Blache D. Glucose and free radicals impair the antioxidant properties of serum albumin. Faseb J 1999;13:233-44.

32. Cai W, He JC, Zhu L, Peppa M, Lu C, Uribarri J, et al. High levels of dietary advanced glycation end products transform low-density lipoprotein into a potent redox-sensitive mitogen-activated protein kinase stimulant in diabetic patients. Circulation 2004;110:285-91.

33. Patel R, Baker SS, Liu W, Desai S, Alkhouri R, Kozielski R, et al. Effect of dietary advanced glycation end products on mouse liver. PLoS One 2012;7:e35143.

34. Paolisso G, Gambardella A, Tagliamonte MR, Saccomanno F, Salvatore T, Gualdiero P, et al. Does free fatty acid infusion impair insulin action also through an increase in oxidative stress? J Clin Endocrinol Metab 1996;81:4244-8.

35. Itabe H, Obama T, Kato R. The Dynamics of Oxidized LDL during Atherogenesis. J Lipids 2011;2011:418313.

36. Groeneweg M, Kanters E, Vergouwe MN, Duerink H, Kraal G, Hofker MH, et al. Lipopolysaccharideinduced gene expression in murine macrophages is enhanced by prior exposure to oxLDL. J Lipid Res 2006;47:2259-67.

37. Liao F, Andalibi A, deBeer FC, Fogelman AM, Lusis AJ. Genetic control of inflammatory gene induction and NF-kappa B-like transcription factor activation in response to an atherogenic diet in mice. J Clin Invest 1993;91:2572-9.

38. Stroka KM, Levitan I, Aranda-Espinoza H. OxLDL and substrate stiffness promote neutrophil transmigration by enhanced endothelial cell contractility and ICAM-1. J Biomech 2012;45:1828-34.

39. Sedgwick JB, Hwang YS, Gerbyshak HA, Kita H, Busse WW. Oxidized low-density lipoprotein activates migration and degranulation of human granulocytes. Am J Respir Cell Mol Biol 2003;29:702-9.

40. McMurray HF, Parthasarathy S, Steinberg D. Oxidatively modified low density lipoprotein is a chemoattractant for human T lymphocytes. J Clin Invest 1993;92:1004-8.

41. Ou HC, Lee WJ, Lee IT, Chiu TH, Tsai KL, Lin CY, et al. Ginkgo biloba extract attenuates oxLDL-induced oxidative functional damages in endothelial cells. J Appl Physiol 2009;106:1674-85.

42. Keiper T, Al-Fakhri N, Chavakis E, Athanasopoulos AN, Isermann B, Herzog S, et al. The role of junctional adhesion molecule-C (JAM-C) in oxidized LDL-mediated leukocyte recruitment. Faseb J 2005;19:2078-80.

43. Lehr HA, Krombach F, Munzing S, Bodlaj R, Glaubitt SI, Seiffge D, et al. In vitro effects of oxidized low density lipoprotein on CD11b/CD18 and L-selectin presentation on neutrophils and monocytes with relevance for the in vivo situation. Am J Pathol 1995;146:218-27. 
44. Kim CS, Kang JH, Cho HR, Blankenship TN, Erickson KL, Kawada T, et al. Potential involvement of CCL23 in atherosclerotic lesion formation/progression by the enhancement of chemotaxis, adhesion molecule expression, and MMP-2 release from monocytes. Inflamm Res 2011;60:889-95.

45. Chen XP, Xun KL, Wu Q, Zhang TT, Shi JS, Du GH. Oxidized low density lipoprotein receptor-1 mediates oxidized low density lipoprotein-induced apoptosis in human umbilical vein endothelial cells: role of reactive oxygen species. Vascul Pharmacol 2007;47:1-9.

46. Li D, Liu L, Chen H, Sawamura T, Ranganathan S, Mehta JL. LOX-1 mediates oxidized low-density lipoprotein-induced expression of matrix metalloproteinases in human coronary artery endothelial cells. Circulation 2003;107:612-7.

47. Wang Y, Ausman LM, Russell RM, Greenberg AS, Wang XD. Increased apoptosis in high-fat dietinduced nonalcoholic steatohepatitis in rats is associated with c-Jun $\mathrm{NH2}$-terminal kinase activation and elevated proapoptotic Bax. J Nutr 2008;138:1866-71.

48. Feldstein AE, Canbay A, Angulo P, Taniai M, Burgart L, Lindor KD, et al. Hepatocyte apoptosis and fas expression are prominent features of human nonalcoholic steatohepatitis. Gastroenterology 2003;125:437-43.

49. Takarada S, Imanishi T, Hano T, Nishio I. Oxidized low-density lipoprotein sensitizes human vascular smooth muscle cells to FAS (CD95)-mediated apoptosis. Clin Exp Pharmacol Physiol 2003;30:289-94.

50. Chang MK, Binder CJ, Miller YI, Subbanagounder G, Silverman GJ, Berliner JA, et al. Apoptotic cells with oxidation-specific epitopes are immunogenic and proinflammatory. J Exp Med 2004;200: 1359-70.

51. Jerome WG, Cox BE, Griffin EE, Ullery JC. Lysosomal cholesterol accumulation inhibits subsequent hydrolysis of lipoprotein cholesteryl ester. Microsc Microanal 2008;14:138-49.

52. Schmitz G, Grandl M. Endolysosomal phospholipidosis and cytosolic lipid droplet storage and release in macrophages. Biochim Biophys Acta 2009;1791:524-39.

53. Weissmann G. The role of lysosomes in inflammation and disease. Annu Rev Med 1967;18:97-112.

54. Duewell P, Kono H, Rayner KJ, Sirois CM, Vladimer G, Bauernfeind FG, et al. NLRP3 inflammasomes are required for atherogenesis and activated by cholesterol crystals. Nature 2010;464:1357-61.

55. Saitoh T, Akira S. Regulation of innate immune responses by autophagy-related proteins. J Cell Biol 2010;189:925-35.

56. Kolios G, Valatas V, Kouroumalis E. Role of Kupffer cells in the pathogenesis of liver disease. World J Gastroenterol 2006;12:7413-20.

57. Day CP, James OF. Steatohepatitis: a tale of two "hits"? Gastroenterology 1998;114:842-5.

58. Tilg $\mathrm{H}$, Moschen AR. Evolution of inflammation in nonalcoholic fatty liver disease: the multiple parallel hits hypothesis. Hepatology 2010;52:1836-46.

59. Bieghs V, Wouters K, van Gorp PJ, Gijbels MJ, de Winther MP, Binder CJ, et al. Role of scavenger receptor $A$ and $\mathrm{CD} 36$ in diet-induced nonalcoholic steatohepatitis in hyperlipidemic mice. Gastroenterology 2010;138:2477-2486, 2486 e2471-3.

60. Leroux A, Ferrere G, Godie V, Cailleux F, Renoud ML, Gaudin F, et al. Toxic lipids stored by Kupffer cells correlates with their pro-inflammatory phenotype at an early stage of steatohepatitis. J Hepatol 2012;57:141-9.

61. Li AC, Glass CK. The macrophage foam cell as a target for therapeutic intervention. Nat Med 2002;8:1235-42.

62. Kunjathoor VV, Febbraio M, Podrez EA, Moore KJ, Andersson L, Koehn S, et al. Scavenger receptors class $\mathrm{A}-\mathrm{I} / \mathrm{II}$ and $\mathrm{CD} 36$ are the principal receptors responsible for the uptake of modified low density lipoprotein leading to lipid loading in macrophages. J Biol Chem 2002;277:49982-8.

63. Yoshida H, Quehenberger O, Kondratenko N, Green S, Steinberg D. Minimally oxidized low-density lipoprotein increases expression of scavenger receptor $A, C D 36$, and macrosialin in resident mouse peritoneal macrophages. Arterioscler Thromb Vasc Biol 1998;18:794-802.

64. Yimin, Furumaki H, Matsuoka S, Sakurai T, Kohanawa M, Zhao S, et al. A novel murine model for nonalcoholic steatohepatitis developed by combination of a high-fat diet and oxidized low-density lipoprotein. Lab Invest 2011;92:265-81.

65. Naito M, Kodama T, Matsumoto A, Doi T, Takahashi K. Tissue distribution, intracellular localization, and in vitro expression of bovine macrophage scavenger receptors. Am J Pathol 1991;139:1411-23. 
66. Bieghs V, Verheyen F, van Gorp PJ, Hendrikx T, Wouters K, Lutjohann D, et al. Internalization of modified lipids by CD36 and SR-A leads to hepatic inflammation and lysosomal cholesterol storage in Kupffer cells. PLoS One 2012;7:e34378.

67. Bieghs V, Van Gorp PJ, Wouters K, Hendrikx T, Gijbels MJ, van Bilsen M, et al. LDL receptor knock-out mice are a physiological model particularly vulnerable to study the onset of inflammation in nonalcoholic fatty liver disease. PLoS One 2012;7:e30668.

68. Griffin EE, Ullery JC, Cox BE, Jerome WG. Aggregated LDL and lipid dispersions induce lysosomal cholesteryl ester accumulation in macrophage foam cells. J Lipid Res 2005;46:2052-60.

69. Bieghs V, van Gorp PJ, Walenbergh S, Gijbels MJ, Verheyen F, Buurman WA, et al. Specific immunization strategies against oxidized LDL: A novel way to reduce non-alcoholic steatohepatitis in mice. Hepatology 2012;56:894-903.

70. Jerome WG. Advanced atherosclerotic foam cell formation has features of an acquired lysosomal storage disorder. Rejuvenation Res 2006;9:245-55.

71. Shaw PX, Horkko S, Chang MK, Curtiss LK, Palinski W, Silverman GJ, et al. Natural antibodies with the T15 idiotype may act in atherosclerosis, apoptotic clearance, and protective immunity. J Clin Invest 2000;105:1731-40.

72. Miller YI, Choi SH, Wiesner P, Fang L, Harkewicz R, Hartvigsen K, et al. Oxidation-specific epitopes are danger-associated molecular patterns recognized by pattern recognition receptors of innate immunity. Circ Res 2011;108:235-48.

73. Briles DE, Forman C, Hudak S, Claflin JL. Anti-phosphorylcholine antibodies of the T15 idiotype are optimally protective against Streptococcus pneumoniae. J Exp Med 1982;156:1177-85.

74. Roberts RA, Ganey PE, Ju C, Kamendulis LM, Rusyn I, Klaunig JE. Role of the Kupffer cell in mediating hepatic toxicity and carcinogenesis. Toxicol Sci 2007;96:2-15.

75. Yamashina S, Takei Y, Ikejima K, Enomoto N, Kitamura T, Sato N. Ethanol-induced sensitization to endotoxin in Kupffer cells is dependent upon oxidative stress. Alcohol Clin Exp Res 2005;29:246S-50S.

76. Holt MP, Ju C. Mechanisms of drug-induced liver injury. Aaps J 2006;8:E48-54.

77. Hoebe KH, Witkamp RF, Fink-Gremmels J, Van Miert AS, Monshouwer M. Direct cell-to-cell contact between Kupffer cells and hepatocytes augments endotoxin-induced hepatic injury. Am J Physiol Gastrointest Liver Physiol 2001;280:G720-8.

78. Canbay A, Feldstein AE, Higuchi H, Werneburg N, Grambihler A, Bronk SF, et al. Kupffer cell engulfment of apoptotic bodies stimulates death ligand and cytokine expression. Hepatology 2003;38:1188-98.

79. Tacke F, Luedde T, Trautwein C. Inflammatory pathways in liver homeostasis and liver injury. Clin Rev Allergy Immunol 2009;36:4-12.

80. Farrell GC, van Rooyen D, Gan L, Chitturi S. NASH is an Inflammatory Disorder: Pathogenic, Prognostic and Therapeutic Implications. Gut Liver 2012;6:149-71.

81. Miura K, Yang L, van Rooijen N, Ohnishi H, Seki E. Hepatic recruitment of macrophages promotes nonalcoholic steatohepatitis through CCR2. Am J Physiol Gastrointest Liver Physiol 2012;302: G1310-21.

82. Pessayre D. Role of mitochondria in non-alcoholic fatty liver disease. J Gastroenterol Hepatol 2007;22 Suppl 1:S20-7.

83. Klebanoff SJ. Myeloperoxidase: friend and foe. J Leukoc Biol 2005;77:598-625.

84. Podrez EA, Schmitt D, Hoff HF, Hazen SL. Myeloperoxidase-generated reactive nitrogen species convert LDL into an atherogenic form in vitro. J Clin Invest 1999;103:1547-60.

85. Rensen SS, Slaats Y, Nijhuis J, Jans A, Bieghs V, Driessen A, et al. Increased hepatic myeloperoxidase activity in obese subjects with nonalcoholic steatohepatitis. Am J Pathol 2009;175:1473-82.

86. Hoppe G, O'Neil J, Sayre LM, Hoff HF. Non-conventional modification of low density lipoproteins: chemical models for macrophage recognition of oxidized LDL. Biochim Biophys Acta 1997;1362:103-8.

87. Thong-Ngam D, Samuhasaneeto S, Kulaputana O, Klaikeaw N. N-acetylcysteine attenuates oxidative stress and liver pathology in rats with non-alcoholic steatohepatitis. World J Gastroenterol 2007;13:5127-32.

88. Abdelmegeed MA, Banerjee A, Yoo SH, Jang S, Gonzalez FJ, Song BJ. Critical role of cytochrome P450 2E1 (CYP2E1) in the development of high fat-induced non-alcoholic steatohepatitis. J Hepatol 2012;57:860-6. 
89. Koruk M, Taysi S, Savas MC, Yilmaz O, Akcay F, Karakok M. Oxidative stress and enzymatic antioxidant status in patients with nonalcoholic steatohepatitis. Ann Clin Lab Sci 2004;34:57-62.

90. Sumida Y, Nakashima T, Yoh T, Furutani M, Hirohama A, Kakisaka Y, et al. Serum thioredoxin levels as a predictor of steatohepatitis in patients with nonalcoholic fatty liver disease. J Hepatol 2003;38:32-8.

91. Chalasani N, Deeg MA, Crabb DW. Systemic levels of lipid peroxidation and its metabolic and dietary correlates in patients with nonalcoholic steatohepatitis. Am J Gastroenterol 2004;99:1497-502.

92. Chalasani N, Gorski JC, Asghar MS, Asghar A, Foresman B, Hall SD, et al. Hepatic cytochrome P450 2E1 activity in nondiabetic patients with nonalcoholic steatohepatitis. Hepatology 2003;37:544-50.

93. Yla-Herttuala S, Rosenfeld ME, Parthasarathy S, Glass CK, Sigal E, Witztum JL, et al. Colocalization of 15-lipoxygenase mRNA and protein with epitopes of oxidized low density lipoprotein in macrophagerich areas of atherosclerotic lesions. Proc Natl Acad Sci U S A 1990;87:6959-63.

94. Mukhopadhyay CK, Fox PL. Ceruloplasmin copper induces oxidant damage by a redox process utilizing cell-derived superoxide as reductant. Biochemistry 1998;37:14222-9.

95. Puri P, Wiest MM, Cheung O, Mirshahi F, Sargeant C, Min HK, et al. The plasma lipidomic signature of nonalcoholic steatohepatitis. Hepatology 2009;50:1827-38.

96. Baskol G, Baskol M, Kocer D. Oxidative stress and antioxidant defenses in serum of patients with nonalcoholic steatohepatitis. Clin Biochem 2007;40:776-80.

97. Videla LA, Rodrigo R, Orellana M, Fernandez V, Tapia G, Quinones L, et al. Oxidative stress-related parameters in the liver of non-alcoholic fatty liver disease patients. Clin Sci (Lond) 2004;106:261-8.

98. Erhardt A, Stahl W, Sies H, Lirussi F, Donner A, Haussinger D. Plasma levels of vitamin E and carotenoids are decreased in patients with Nonalcoholic Steatohepatitis (NASH). Eur J Med Res 2011;16:76-8.

99. Hardwick RN, Fisher CD, Canet MJ, Lake AD, Cherrington NJ. Diversity in antioxidant response enzymes in progressive stages of human nonalcoholic fatty liver disease. Drug Metab Dispos 2010;38:2293-301.

100. Haque JA, McMahan RS, Campbell JS, Shimizu-Albergine M, Wilson AM, Botta D, et al. Attenuated progression of diet-induced steatohepatitis in glutathione-deficient mice. Lab Invest 2010;90: 1704-17.

101. Tobkes Al, Nord HJ. Liver biopsy: review of methodology and complications. Dig Dis 1995;13:267-74.

102. Wieckowska A, McCullough AJ, Feldstein AE. Noninvasive diagnosis and monitoring of nonalcoholic steatohepatitis: present and future. Hepatology 2007;46:582-9.

103. Shen J, Chan HL, Wong GL, Choi PC, Chan AW, Chan HY, et al. Non-invasive diagnosis of non-alcoholic steatohepatitis by combined serum biomarkers. J Hepatol 2012;56:1363-70.

104. Agarwal N, Sharma BC. Insulin resistance and clinical aspects of non-alcoholic steatohepatitis (NASH). Hepatol Res 2005;33:92-6.

105. Toshima S, Hasegawa A, Kurabayashi M, Itabe H, Takano T, Sugano J, et al. Circulating oxidized low density lipoprotein levels. A biochemical risk marker for coronary heart disease. Arterioscler Thromb Vasc Biol 2000;20:2243-7.

106. Binder CJ, Horkko S, Dewan A, Chang MK, Kieu EP, Goodyear CS, et al. Pneumococcal vaccination decreases atherosclerotic lesion formation: molecular mimicry between Streptococcus pneumoniae and oxidized LDL. Nat Med 2003;9:736-43.

107. Chang MK, Bergmark C, Laurila A, Horkko S, Han KH, Friedman P, et al. Monoclonal antibodies against oxidized low-density lipoprotein bind to apoptotic cells and inhibit their phagocytosis by elicited macrophages: evidence that oxidation-specific epitopes mediate macrophage recognition. Proc Natl Acad Sci U S A 1999;96:6353-8.

108. Inoue T, Uchida T, Kamishirado H, Takayanagi K, Hayashi T, Morooka S. Clinical significance of antibody against oxidized low density lipoprotein in patients with atherosclerotic coronary artery disease. J Am Coll Cardiol 2001;37:775-9.

109. Salonen JT, Yla-Herttuala S, Yamamoto R, Butler S, Korpela H, Salonen R, et al. Autoantibody against oxidised LDL and progression of carotid atherosclerosis. Lancet 1992;339:883-7.

110. Mayr M, Kiechl S, Tsimikas S, Miller E, Sheldon J, Willeit J, et al. Oxidized low-density lipoprotein autoantibodies, chronic infections, and carotid atherosclerosis in a population-based study. J Am Coll Cardiol 2006;47:2436-43.

111. de Geest B, Collen D. Antibodies against oxidized LDL for non-invasive diagnosis of atherosclerotic vascular disease. Eur Heart J 2001;22:1517-8. 
112. Caligiuri G, Khallou-Laschet J, Vandaele M, Gaston AT, Delignat S, Mandet C, et al. Phosphorylcholinetargeting immunization reduces atherosclerosis. J Am Coll Cardiol 2007;50:540-6.

113. Uygun A, Kadayifci A, Yesilova Z, Erdil A, Yaman H, Saka M, et al. Serum leptin levels in patients with nonalcoholic steatohepatitis. Am J Gastroenterol 2000;95:3584-9.

114. Ishii $\mathrm{H}$, Horie $\mathrm{Y}$, Ohshima S, Anezaki $\mathrm{Y}$, Kinoshita N, Dohmen $\mathrm{T}$, et al. Eicosapentaenoic acid ameliorates steatohepatitis and hepatocellular carcinoma in hepatocyte-specific Pten-deficient mice. J Hepatol 2009;50:562-71.

115. Tanaka N, Sano K, Horiuchi A, Tanaka E, Kiyosawa K, Aoyama T. Highly purified eicosapentaenoic acid treatment improves nonalcoholic steatohepatitis. J Clin Gastroenterol 2008;42:413-8.

116. Shiri-Sverdlov R, Wouters K, van Gorp PJ, Gijbels MJ, Noel B, Buffat L, et al. Early diet-induced nonalcoholic steatohepatitis in APOE2 knock-in mice and its prevention by fibrates. J Hepatol 2006;44:732-41.

117. Laurin J, Lindor KD, Crippin JS, Gossard A, Gores GJ, Ludwig J, et al. Ursodeoxycholic acid or clofibrate in the treatment of non-alcohol-induced steatohepatitis: a pilot study. Hepatology 1996;23:1464-7.

118. Basaranoglu $\mathrm{M}$, Acbay $\mathrm{O}$, Sonsuz A. A controlled trial of gemfibrozil in the treatment of patients with nonalcoholic steatohepatitis. J Hepatol 1999;31:384.

119. Hyogo H, Tazuma S, Arihiro K, Iwamoto K, Nabeshima Y, Inoue M, et al. Efficacy of atorvastatin for the treatment of nonalcoholic steatohepatitis with dyslipidemia. Metabolism 2008;57:1711-8.

120. Kiyici M, Gulten M, Gurel S, Nak SG, Dolar E, Savci G, et al. Ursodeoxycholic acid and atorvastatin in the treatment of nonalcoholic steatohepatitis. Can J Gastroenterol 2003;17:713-8.

121. Georgescu EF, Georgescu M. Therapeutic options in non-alcoholic steatohepatitis (NASH). Are all agents alike? Results of a preliminary study. J Gastrointestin Liver Dis 2007;16:39-46.

122. Resch U, Tatzber F, Budinsky A, Sinzinger $H$. Reduction of oxidative stress and modulation of autoantibodies against modified low-density lipoprotein after rosuvastatin therapy. $\mathrm{Br} \mathrm{J}$ Clin Pharmacol 2006;61:262-74.

123. Ekstedt M, Franzen LE, Mathiesen UL, Thorelius L, Holmqvist M, Bodemar G, et al. Long-term followup of patients with NAFLD and elevated liver enzymes. Hepatology 2006;44:865-73.

124. Sanyal AJ, Chalasani N, Kowdley KV, McCullough A, Diehl AM, Bass NM, et al. Pioglitazone, vitamin E, or placebo for nonalcoholic steatohepatitis. N Engl J Med 2010;362:1675-85.

125. Zein CO, Lopez R, Kirwan JP, Yerian LM, McCullough AJ, Hazen SL, et al. Pentoxifylline decreases oxidized lipid products in nonalcoholic steatohepatitis: New evidence on the potential therapeutic mechanism. Hepatology 2012;56:1291-9.

126. Ricciarelli R, Zingg JM, Azzi A. Vitamin E reduces the uptake of oxidized LDL by inhibiting CD36 scavenger receptor expression in cultured aortic smooth muscle cells. Circulation 2000;102:82-7.

127. Van Wagner LB, Koppe SW, Brunt EM, Gottstein J, Gardikiotes K, Green RM, et al. Pentoxifylline for the treatment of non-alcoholic steatohepatitis: a randomized controlled trial. Ann Hepatol 2011;10:277-86.

128. Lavine JE, Schwimmer JB, Van Natta ML, Molleston JP, Murray KF, Rosenthal P, et al. Effect of vitamin E or metformin for treatment of nonalcoholic fatty liver disease in children and adolescents: the TONIC randomized controlled trial. Jama 2011;305:1659-68.

129. Ratziu V, Charlotte F, Heurtier A, Gombert S, Giral P, Bruckert E, et al. Sampling variability of liver biopsy in nonalcoholic fatty liver disease. Gastroenterology 2005;128:1898-906.

130. Pietu F, Guillaud O, Walter T, Vallin M, Hervieu V, Scoazec JY, et al. Ursodeoxycholic acid with vitamin $\mathrm{E}$ in patients with nonalcoholic steatohepatitis: long-term results. Clin Res Hepatol Gastroenterol 2012;36:146-55.

131. Lindor KD, Kowdley KV, Heathcote EJ, Harrison ME, Jorgensen R, Angulo P, et al. Ursodeoxycholic acid for treatment of nonalcoholic steatohepatitis: results of a randomized trial. Hepatology 2004;39: 770-8.

132. Park HJ, Lee JY, Chung MY, Park YK, Bower AM, Koo SI, et al. Green tea extract suppresses NFkappaB activation and inflammatory responses in diet-induced obese rats with nonalcoholic steatohepatitis. J Nutr 2012;142:57-63.

133. Rezazadeh A, Yazdanparast R, Molaei M. Amelioration of diet-induced nonalcoholic steatohepatitis in rats by $\mathrm{Mn}$-salen complexes via reduction of oxidative stress. J Biomed Sci 2012;19:26. 
134. Abdelmalek MF, Angulo $P$, Jorgensen RA, Sylvestre PB, Lindor KD. Betaine, a promising new agent for patients with nonalcoholic steatohepatitis: results of a pilot study. Am J Gastroenterol 2001;96: 2711-7.

135. Finn AV, Nakano M, Polavarapu R, Karmali V, Saeed O, Zhao X, et al. Hemoglobin directs macrophage differentiation and prevents foam cell formation in human atherosclerotic plaques. J Am Coll Cardiol 2012;59:166-77. 


\section{Chapter 3}

\section{Trapping of oxidized LDL in lysosomes of Kupffer cells is a trigger for hepatic inflammation}

V Bieghs, SMA Walenbergh, T Hendrikx, PJ van Gorp, F Verheyen, SW Olde Damink, AA Masclee, GH Koek, MH Hofker, CJ Binder, R Shiri-Sverdlov Liver Int 2013;33:1056-61 


\section{Abstract}

\section{Background and aims}

Non-alcoholic steatohepatitis (NASH) is characterized by steatosis and inflammation. The transition from steatosis towards NASH represents a key step in pathogenesis, as it will set the stage for further liver damage. Under normal conditions, lipoproteins that are endocytosed by Kupffer cells (KCS) are easily transferred from the lysosomes into the cytoplasm. Oxidized LDL (oxLDL) that is taken up by macrophages in vitro is trapped within the lysosomes, while acetylated LDL (acLDL) is leading to normal lysosomal hydrolysis, resulting in cytoplasmic storage. We have recently demonstrated that hepatic inflammation is correlated with lysosomal trapping of lipids. So far, a link between lysosomal trapping of oxLDL and inflammation was not established. We hypothesized that lysosomal trapping of oxLDL in KCs will lead to hepatic inflammation.

\section{Methods}

$L d r^{-/-}$mice were injected with LDL, acLDL and oxLDL and sacrificed after 2, 6 and 24 hours.

\section{Results}

Electron microscopy of KCs demonstrated that after oxLDL injection, small lipid inclusions were present inside the lysosomes after all time points and were mostly pronounced after 6 and 24 hours. In contrast, no lipid inclusions were present inside after LDL or acLDL injection. Hepatic expression of several inflammatory genes and scavenger receptors was higher after oxLDL injections compared to LDL or acLDL.

\section{Conclusions}

These data suggest that trapping of oxLDL inside lysosomes of KCs in vivo is causally linked to increased hepatic inflammatory gene expression. Our novel observations provide new bases for the prevention and treatment of NASH. 


\section{Introduction}

Non-alcoholic fatty liver disease (NAFLD) is a condition ranging from benign lipid accumulation in the liver (steatosis) to steatosis combined with inflammation. The latter is referred to as non-alcoholic steatohepatitis (NASH) and is viewed as the hepatic event of the metabolic syndrome. As obesity and insulin resistance reach epidemic proportions in industrialized countries, the prevalence of NASH is increasing and is therefore considered a major health hazard. Although steatosis alone is considered as the less aggressive stage of NAFLD, inflammation represents a key step in the pathogenesis of NASH, thereby setting the stage for further liver damage including fibrosis, cirrhosis and liver cancer. ${ }^{1}$ Currently, the mechanisms by which inflammation develops are poorly understood and therapy options are very poor.

Recent studies point strongly towards the importance of Kupffer cells (KCs), the resident macrophages of the liver, in triggering hepatic inflammation. ${ }^{2}$ In line with this view, we demonstrated the correlation between hepatic inflammation and the appearance of foamy KCs, analogous to foamy macrophages in atherosclerosis. ${ }^{3}$ Such cholesterol-loaded foamy macrophages are formed by the uptake of oxidized cholesterol-rich low density lipoproteins (oxLDL) via scavenger receptors. ${ }^{4}$ Uptake of oxLDL by macrophages in vitro is shown to be resistant to rapid endolysosomal hydrolysis and is trapped within the lysosomes, while acetylated LDL (acLDL) is leading to normal lysosomal hydrolysis, resulting in cytoplasmic storage of cholesteryl esters. ${ }^{5,6}$ We have previously shown that hematopoietic deletion of CD36 and SR-A, two main scavenger receptors for the uptake of oxLDL, sets off a cascade of proinflammatory events leading to the initiation of the inflammatory response in the liver. $^{7}$ Moreover, the reduced inflammatory response was associated with less lysosomal cholesterol accumulation inside $\mathrm{KCs} .{ }^{8,9}$ However, a causal link between lysosomal cholesterol accumulation in KCs and hepatic inflammation has not yet been established. In the current manuscript, we hypothesize that lysosomal trapping of oxLDL in KCs leads to hepatic inflammation.

To investigate whether oxLDL can directly affect hepatic inflammation in vivo, LdIr $^{-1}$ mice were injected with a bolus of oxLDL and sacrificed after 2, 6 and 24 hours of injection. Injections with PBS, LDL and acLDL were used as controls. After oxLDL injection, we found that lysosomal trapping of oxLDL was correlated with elevated expression of hepatic inflammatory genes. These data suggest a causal relationship between oxLDL and hepatic inflammation and provide new bases for the prevention and treatment of NASH. 


\section{Materials and methods}

\section{Mice}

Mice were housed under standard conditions and given free access to food and water. Experiments were performed according to Dutch regulations and approved by the Committee for Animal Welfare of Maastricht University. Twelve-week-old female LDL receptor knockout mice were injected in the tail vein with a bolus of human LDL,

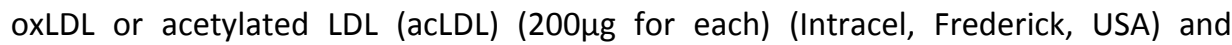
sacrificed after 2, 6 and 24 hours of injection ( $n=8$ per group). The control group was injected with PBS. The mice were sacrificed by cervical dislocation. Tissues were then isolated and snap-frozen in liquid nitrogen and stored at $-80^{\circ} \mathrm{C}$.

\section{RNA isolation and quantitative PCR}

RNA isolation and cDNA synthesis were performed as described previously. ${ }^{3,7}$ All applications were performed according to the manufacturers' protocols. For each gene, a standard curve was generated with a serial dilution of a liver cDNA pool. To standardize for the amount of cDNA, Cyclophillin A (Ppia) was used as the reference gene. Primer sets for the selected genes were developed with Primer Express version 2.0 (Applied Biosystems, Foster City, CA, USA) using default settings. Primer sequences were as followed: Ppia forward 5'-TTCCTCCTTTCACAGAATTATTCCA; Ppia reverse 3'-CCGCCAGTGCCATTATGG; Tnf forward 5'-CATCTTCTCAAAATTCGAGTGACAA; Tnf reverse 3'-TGGGAGTAGACAAGGTACAACCC; Saa-1 forward 5' GGCTGCTGAGAAAATCAGTGATG; Saa-1 reverse 3'-TCAGCAATGGTGTCCTCATGTC; T/r-4 forward 5'-TATCCAGGTGTGAAATTGAAACAATT; T/r-4 reverse 3'-GGGTTTCCTGTCAGTATCAAGTTTG; Icam forward 5'-CTACCATCACCGTGTATTCGTTTC; Icam reverse 3'CGGTGCTCCACCATCCA; Hmox forward 5'- CCGCCTTCCTGCTCAACAT; Hmox reverse 3'ATCTGTGAGGGACTCTGGTCTTTG; Cd36 forward 5'- GCCAAGCTATTGCGACATGA; Cd36 reverse 3'-AAAAGAATCTCAATGTCCGAGACTTT; $S r$ - $a$ forward 5'-CATACAGAAACACTGCATGTCAGAGT; Sr-A reverse 3'-TTCTGCTGATACTTTGTACACACGTT. Data from qPCR was analyzed according to the relative standard curve method.

\section{Liver histology}

A detailed overview about liver histology was described previously. ${ }^{7}$ In brief, frozen liver sections $(7 \mu \mathrm{m})$ were fixed in acetone and stained with rat-anti-mouse Mac1 (M1/70) (generous gifts from Prof. Kraal, Free University, Amsterdam, the Netherlands). Immune cells were counted in six microscopically views (magnification 200x) and were noted as cells $/ \mathrm{mm}^{2}$. Pictures were taken with a Nikon DMX1200 digital camera and ACT-1 version 2.63 software (Nikon Instruments Europe, Amstelveen, the Netherlands). 


\section{Electron microscopy}

Livers were freshly isolated from the mice and perfused and fixed overnight with $2.5 \%$ glutaraldehyde (Ted Pella, Redding, CA, USA). Tissue fragments were washed and post-fixed in $1 \%$ osmium tetroxide. Tissues were subsequently dehydrated through $100 \%$ ethanol, cleared with propylene oxide, and embedded in epoxy resin. Next, sections of 70-90 nm were cut on an ultra-microtome, mounted on Formvar-coated (1595E, Merck) 75-mesh copper grids and counterstained with uranyl acetate and lead citrate before analysis on a Philips CM100 transmission electron microscope.

\section{Statistics}

Data were analyzed using Graphpad Prism 4.0.3 software. Groups were compared using the unpaired t-test. The data were expressed as the mean and standard error of the mean and were considered significantly different at ${ }^{*} \mathrm{p}<0.05$; $* * \mathrm{p}<0.01$; or $* * * \mathrm{p}<0.001$.

\section{Results}

\section{The injected oxLDL is trapped inside lysosomes of Kupffer cells}

The direct role of oxLDL in hepatic inflammation was investigated by intravenous injection of oxLDL in Ldlr/- mice. Normal LDL, acLDL and PBS were used as control. Electron microscopy was performed to explore the intracellular distribution of oxLDL, LDL and acLDL inside KCs (Figure 3.1A-C). After oxLDL injection, small lipid inclusions were present inside the lysosomes of $\mathrm{KCs}$ in all time points and were mostly pronounced after 6 and 24h of oxLDL injection (Figure 3.1A). LDL could not be detected inside the KCs after all time points (Figure 3.1B), while acLDL was mainly located inside the endothelial cells of the liver after 2 and 6 hours of injection (Figure 3.1D), instead of in KCs (Figure 3.1C). Altogether, these data demonstrate that oxLDL is trapped inside lysosomes of KCs in vivo.

\section{Hepatic inflammation is increased after oxLDL injection}

After 24 hours of injection, hepatic gene expression of the inflammatory markers tumor necrosis factor alpha (Tnf- $\alpha)$, serum amyloid A1 (Saa-1), toll-like receptor 4 $(T / r-4)$ and intracellular adhesion molecule-1 (Icam-1) was significantly higher in mice that received a bolus of oxLDL compared to acLDL (Figure 3.2). The expression of Tnf- $\alpha$ and Saa-1 of oxLDL-injected mice was also higher than LDL-injected mice after $24 \mathrm{~h}$. Moreover, Icam-1 was already significantly increased after $2 \mathrm{~h}$ of oxLDL injection compared with LDL and acLDL. The anti-oxidant heme oxygenase 1 ( $\mathrm{Hmox}-1)$ was also higher after $24 \mathrm{~h}$ of oxLDL injection compared with LDL and acLDL. Infiltration of monocytes/macrophages and neutrophils (Cd11b staining) was not different between 
the groups, which is in line with the unchanged mRNA expression of Integrin alpha-M (Itgam) (Supplemental Figure S3.1). Together, these data demonstrate that the inflammatory gene expression in liver is increased after oxLDL injection compared with LDL and acLDL.

A

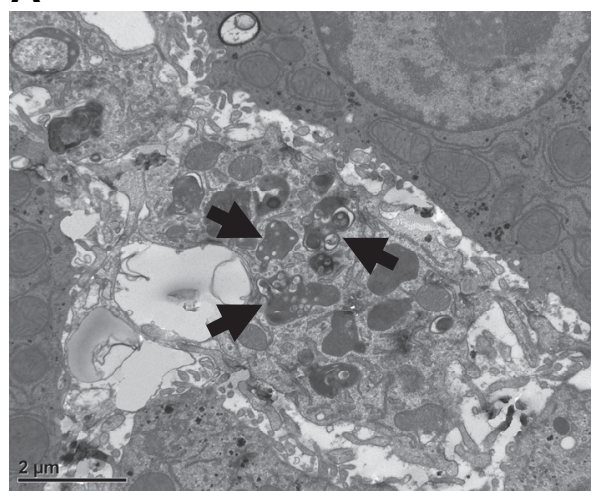

C

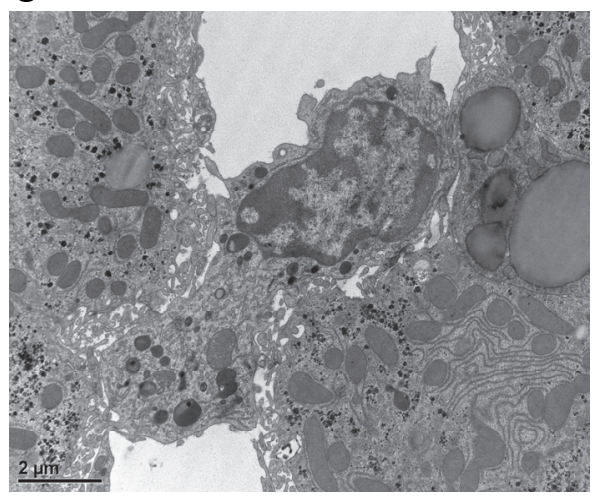

B

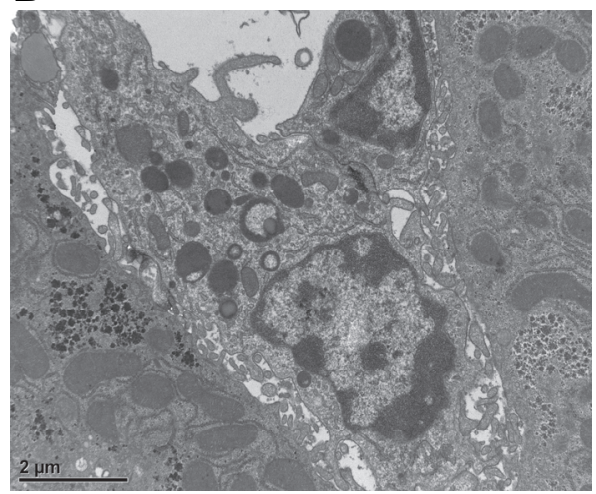

D

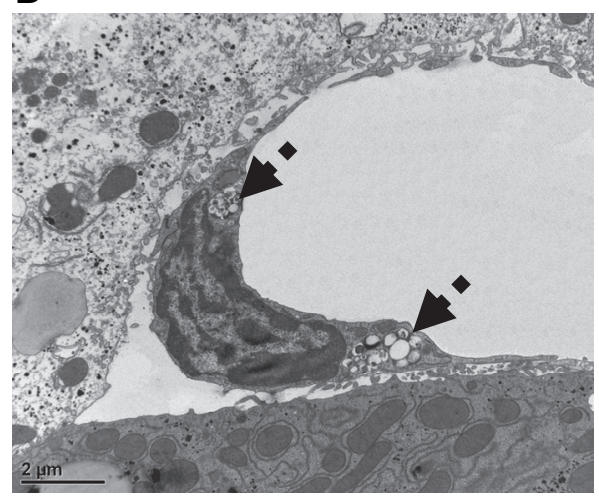

Figure 3.1 Electron microscopy after acLDL, oxLDL and LDL injections. (A-C) Representative electron microscopy pictures of Kupffer cells $24 \mathrm{~h}$ after oxLDL, LDL and acLDL injections respectively. (D) Representative electron microscopy picture of an endothelial cell $2 \mathrm{~h}$ after acLDL injection. OxLDL inside lysosomes of KCs is indicated by solid arrows, acLDL inside endothelial cells by broken arrows.

Next, mRNA expression of the two main scavenger receptors CD36 and SR-A was performed to explore their response upon oxLDL injection. After $24 \mathrm{~h}$ of oxLDL injection, expression of Cd36 was significantly increased compared with LDL and acLDL, while expression of $S r-a$ was increased compared with acLDL. However, after 2 and $6 \mathrm{~h}$ of $\mathrm{LDL}$ injection, expression of $\mathrm{Sr}$ - $a$ was increased compared with oxLDL injection (Figure 3.2). Altogether, these data demonstrate that both scavenger 
receptors CD36 and SR-A are involved in the inflammatory response upon oxLDL administration in vivo (Figure 3.3).
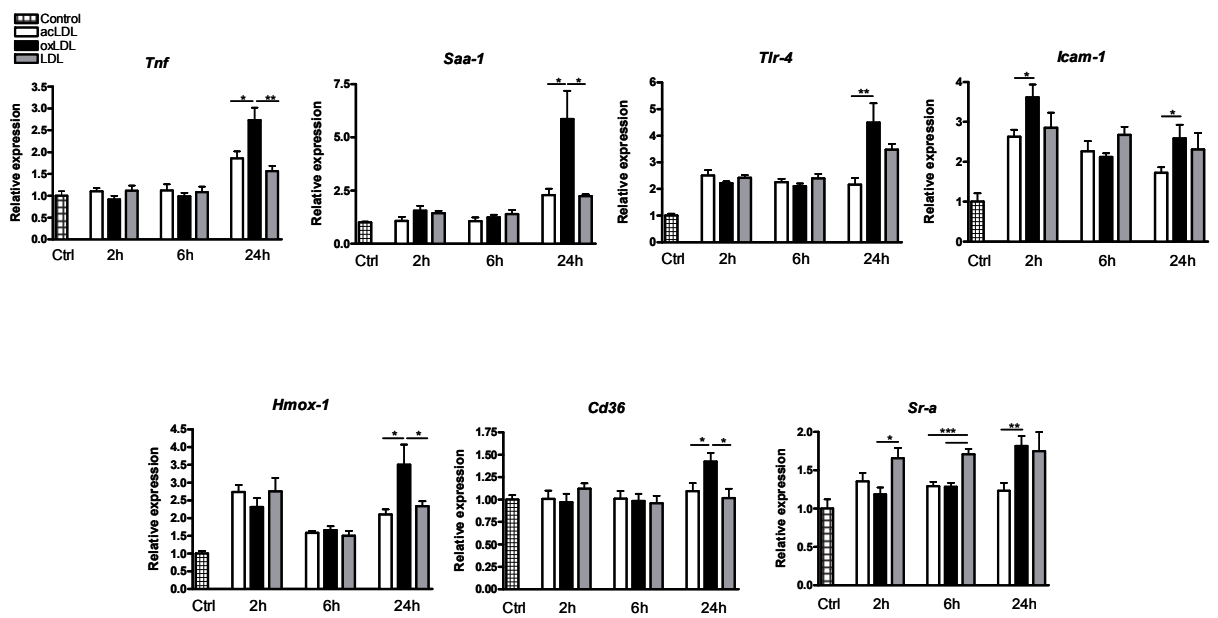

Figure 3.2 Hepatic gene expression after acLDL, oxLDL and LDL injections. Gene expression analysis by qPCR of tumor necrosis factor alpha $(\operatorname{Tnf}-\alpha)$, serum amyloid A1 (Saa-1), toll-like receptor 4 (TIr-4), intracellular adhesion molecule-1 (Icam-1), heme oxygenase 1 (Hmox-1) and scavenger receptors $\mathrm{Cd} 36$ and $\mathrm{Sr}-\mathrm{a}$. Gene expression data are shown relative to the control-injected group. ${ }^{*}$ indicates $p<0.05,{ }^{* *} p<0.01$ and $* * * p<0.001$.

\section{Normal condition}

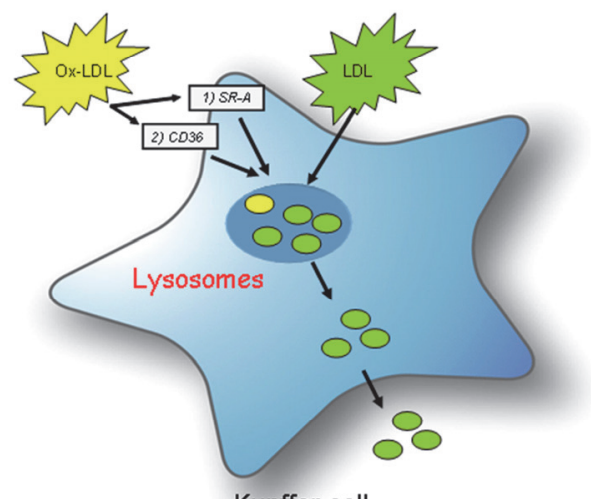

Kupffer cell

\section{Hepatic inflammation}

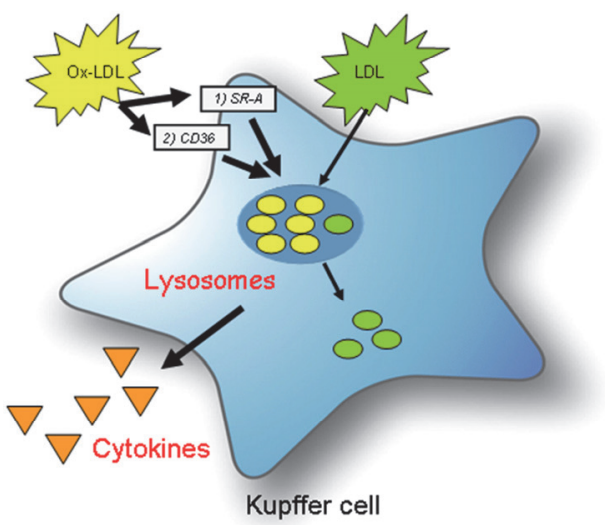

Figure 3.3 Uptake of modified lipoproteins by Kupffer cells (KCs). Under normal conditions, circulating cholesterol is taken up by KCs and initially directed to lysosomes. After hydrolyzation by lysosomal enzymes, free cholesterol is transferred into the cytoplasm. There, free cholesterol can be converted into cholesterol esters, or excreted from the cell by efflux mechanisms. Oxidized LDL (oxLDL) can be taken up by scavenger receptors CD36 and SR-A on the KCs. OxLDL is then directed to the lysosomes of the KCs, where it will accumulate via unknown mechanisms. Therefore, oxLDL cannot be converted into free cholesterol and will be trapped inside the lysosomes, triggering an inflammatory response. 


\section{Discussion}

This study demonstrates for the first time the in vivo effects of oxLDL on hepatic inflammatory gene expression. We have previously shown the association between lysosomal trapping of oxLDL and inflammation. However, a causal link was never established. In the current manuscript, we investigated the specific effect of oxLDL on the development of hepatic inflammation. While injection of normal LDL or acLDL was not associated with lysosomal trapping or inflammation, we demonstrated for the first time that in a mouse model of NASH, oxLDL is trapped within the lysosomes of $\mathrm{KCs}$ and is associated with an inflammatory response. These data provide new bases for the prevention and treatment of NASH.

Oxidative modification of LDL transforms LDL to a pro-inflammatory, immunogenic and cytotoxic oxLDL that is generally held as a key component in the development of atherosclerotic foam cells. ${ }^{10}$ Oxidation of LDL has been found to take place in the arterial wall $^{11}$ and in plasma. ${ }^{12}$ By functioning as a scavenger organ for circulating oxLDL, the liver reduces the harmful effects of oxLDL in other tissues. ${ }^{13}$ However, there is some evidence that high levels of circulating oxLDL can impair the function of the liver itself ${ }^{14}$, and when injected into the general circulation of mice, oxLDL can induce several pathophysiological events in the hepatic sinusoids. ${ }^{15}$ Although these findings support the idea that oxLDL is very harmful for the liver, the authors did not investigate the link with lysosomal cholesterol accumulation.

The clearance of native LDL from serum proceeds at a low rate. ${ }^{13}$ When LDL is oxidized or acetylated and injected into rats, this leads to a markedly increased removal from the blood circulation paralleled with an increased uptake in the liver. ${ }^{13}$ The KC uptake of oxLDL 10 min after injection is much higher than for acLDL, leading to KCs as the main liver site for oxLDL uptake. ${ }^{13}$ Furthermore, oxLDL that is taken up by macrophages in vitro is trapped within the lysosomes, while acLDL is leading to normal lysosomal hydrolysis, resulting in cytoplasmic storage of cholesteryl esters. ${ }^{5,6}$ Therefore, although acLDL is not naturally present in vivo, we have used it in the current experiment as a control for lysosomal cholesterol accumulation achieved by oxLDL. We demonstrated that the injected oxLDL is trapped inside lysosomes of KCs, while acLDL is not present in the cytoplasm of KCs, but is mainly found in the endothelial cells of the liver. These data are also in agreement with previous observations in literature, demonstrating that acLDL is mainly taken up by the endothelial cells in the liver, and not by $\mathrm{KCs} .{ }^{13,16}$ It has been suggested that unlike acLDL, uptake of oxLDL by macrophages leads to impaired lysosomal degradation, ${ }^{17}$ and thereby expansion of and a decrease in the density of the lysosomal compartments in macrophages. ${ }^{18}$ In the current manuscript, we show for the first time that accumulation of oxLDL inside lysosomes of KCs can also trigger an inflammatory response. Although the hepatic inflammatory gene expression was 
already affected after several hours of oxLDL injection, infiltration of inflammatory cells in the liver (CD11b staining) was not observed. This lack of inflammatory cell infiltration is probably related to the rapid clearance of oxLDL from the circulation ${ }^{13}$, and therefore we could only observe changes in the inflammatory gene expression upon injections as this is rapidly influenced. These data are also in line with our expectations, as the accumulation of oxLDL inside the lysosomes will first lead to activation of the KCs, which in turn will lead to a rapid release of a wide range of inflammatory mediators and signalling molecules for the attraction of inflammatory cells. $^{19}$

The two main macrophage scavenger receptors responsible for binding and internalization of oxLDL, namely CD36 and SR-A, showed elevated hepatic mRNA expression upon oxLDL injection. These data are also in line with previous reports on the effect of oxLDL on scavenger receptors, where functional expression of CD36 and SR-A was increased in macrophages upon oxLDL loading. ${ }^{20,21}$ We have previously demonstrated that both the scavenger receptors are involved in lysosomal trapping of oxLDL. ${ }^{9}$ When oxLDL is trapped inside lysosomes because of impaired cholesteryl ester hydrolysis or a change in the acidic $\mathrm{pH}$ of lysosomes ${ }^{22}$, it has the potential to damage lysosomal membranes. ${ }^{23}$ Endocytosed oxLDL particles partially inactivate lysosomal enzymes and cause relocation of these enzymes to the cytosol ${ }^{24}$, as well as activation of the NLRP3 inflammasome. ${ }^{25-27}$ Moreover, when there are high levels of circulating oxLDL, as observed in foamy macrophages found in inflamed atherosclerotic plaques, cholesterol is not transferred into the cytoplasm but rather accumulates inside lysosomes. ${ }^{22,28,29}$ Several lines of evidence also indicate a strong association between lysosomal cholesterol accumulation and inflammation. ${ }^{23,25,27,30,31}$ Our current study is the first one to provide a causal relationship between lysosomal oxLDL trapping and elevation of hepatic inflammatory gene expression in the context of NASH.

Taken altogether, our novel observations point towards the link between lysosomal oxLDL accumulation inside KCs and hepatic inflammatory gene expression. Therefore, inhibition of oxLDL itself or the redirection of lysosomal cholesterol accumulation can provide a new basis for the prevention and treatment of NASH. 


\section{References}

1. Angulo, P., Nonalcoholic fatty liver disease. N Engl J Med 2002;346:1221-31.

2. Kolios, G., V. Valatas and E. Kouroumalis, Role of Kupffer cells in the pathogenesis of liver disease. World J Gastroenterol 2006;12:7413-20.

3. Wouters, K., P.J. van Gorp, V. Bieghs, et al., Dietary cholesterol, rather than liver steatosis, leads to hepatic inflammation in hyperlipidemic mouse models of nonalcoholic steatohepatitis. Hepatology 2008;48:474-86.

4. Hansson, G.K., A.K. Robertson and C. Soderberg-Naucler, Inflammation and atherosclerosis. Annu Rev Pathol 2006;1:297-329.

5. Yancey, P.G. and W.G. Jerome, Lysosomal sequestration of free and esterified cholesterol from oxidized low density lipoprotein in macrophages of different species. J Lipid Res 1998;39:1349-61.

6. Yancey, P.G., S. Miles, J. Schwegel and W.G. Jerome, Uptake and trafficking of mildly oxidized LDL and acetylated LDL in THP-1 cells does not explain the differences in lysosomal metabolism of these two lipoproteins. Microsc Microanal 2002;8:81-93.

7. Bieghs, V., K. Wouters, P.J. van Gorp, et al., Role of scavenger receptor A and CD36 in diet-induced nonalcoholic steatohepatitis in hyperlipidemic mice. Gastroenterology 2010;138:2477-86, 2486 e1-3.

8. Bieghs, V., P.J. van Gorp, S. Walenbergh, et al., Specific immunization strategies against oxidized LDL: A novel way to reduce non-alcoholic steatohepa titis in mice. Hepatology 2012;56:894-903.

9. Bieghs, V., F. Verheyen, P.J. van Gorp, et al., Internalization of Modified Lipids by CD36 and SR-A Leads to Hepatic Inflammation and Lysosomal Cholesterol Storage in Kupffer Cells. PLoS One 2012;7: e34378.

10. Steinberg, D., Low density lipoprotein oxidation and its pathobiological significance. J Biol Chem 1997;272:20963-6.

11. Yla-Herttuala, S., W. Palinski, M.E. Rosenfeld, et al., Evidence for the presence of oxidatively modified low density lipoprotein in atherosclerotic lesions of rabbit and man. J Clin Invest 1989;84:1086-95.

12. Avogaro, P., G.B. Bon and G. Cazzolato, Presence of a modified low density lipoprotein in humans. Arteriosclerosis 1988;8:79-87.

13. Van Berkel, T.J., Y.B. De Rijke and J.K. Kruijt, Different fate in vivo of oxidatively modified low density lipoprotein and acetylated low density lipoprotein in rats. Recognition by various scavenger receptors on Kupffer and endothelial liver cells. J Biol Chem 1991;266:2282-9.

14. Itabe, H., Oxidized low-density lipoproteins: what is understood and what remains to be clarified. Biol Pharm Bull 2003;26:1-9.

15. Oteiza, A., R. Li, R.S. McCuskey, B. Smedsrod and K.K. Sorensen, Effects of oxidized low-density lipoproteins on the hepatic microvasculature. Am J Physiol Gastrointest Liver Physiol 2011;301: G684-93.

16. Nagelkerke, J.F., K.P. Barto and T.J. van Berkel, In vivo and in vitro uptake and degradation of acetylated low density lipoprotein by rat liver endothelial, Kupffer, and parenchymal cells. J Biol Chem 1983;258:12221-7.

17. Yancey, P.G. and W.G. Jerome, Lysosomal cholesterol derived from mildly oxidized low density lipoprotein is resistant to efflux. J Lipid Res 2001;42:317-27.

18. Lougheed, M., E.D. Moore, D.R. Scriven and U.P. Steinbrecher, Uptake of oxidized LDL by macrophages differs from that of acetyl LDL and leads to expansion of an acidic endolysosomal compartment. Arterioscler Thromb Vasc Biol 1999;19:1881-90.

19. Racanelli, V. and B. Rehermann, The liver as an immunological organ. Hepatology 2006;43(2 Suppl 1): S54-62.

20. Yoshida, H., O. Quehenberger, N. Kondratenko, S. Green and D. Steinberg, Minimally oxidized lowdensity lipoprotein increases expression of scavenger receptor A, CD36, and macrosialin in resident mouse peritoneal macrophages. Arterioscler Thromb Vasc Biol 1998;18:794-802.

21. Han, J., D.P. Hajjar, M. Febbraio and A.C. Nicholson, Native and modified low density lipoproteins increase the functional expression of the macrophage class B scavenger receptor, CD36. J Biol Chem 1997;272:21654-9.

22. Schmitz, G. and M. Grandl, Endolysosomal phospholipidosis and cytosolic lipid droplet storage and release in macrophages. Biochim Biophys Acta 2009;1791:524-39. 
23. Yuan, X.M., W. Li, A.G. Olsson and U.T. Brunk, The toxicity to macrophages of oxidized low-density lipoprotein is mediated through lysosomal damage. Atherosclerosis 1997;133:153-61.

24. Li, W., X.M. Yuan, A.G. Olsson and U.T. Brunk, Uptake of oxidized LDL by macrophages results in partial lysosomal enzyme inactivation and relocation. Arterioscler Thromb Vasc Biol 1998;18:177-84.

25. Duewell, P., H. Kono, K.J. Rayner, et al., NLRP3 inflammasomes are required for atherogenesis and activated by cholesterol crystals. Nature 2010;464:1357-61.

26. Hornung, V., F. Bauernfeind, A. Halle, et al., Silica crystals and aluminum salts activate the NALP3 inflammasome through phagosomal destabilization. Nat Immunol 2008;9:847-56.

27. Rajamaki, K., J. Lappalainen, K. Oorni, et al., Cholesterol crystals activate the NLRP3 inflammasome in human macrophages: a novel link between cholesterol metabolism and inflammation. PLoS One 2010;5:e11765.

28. Griffin, E.E., J.C. Ullery, B.E. Cox and W.G. Jerome, Aggregated LDL and lipid dispersions induce lysosomal cholesteryl ester accumulation in macrophage foam cells. J Lipid Res 2005;46:2052-60.

29. Jerome, W.G., Advanced atherosclerotic foam cell formation has features of an acquired lysosomal storage disorder. Rejuvenation Res 2006;9:245-55.

30. Weissmann, G., The role of lysosomes in inflammation and disease. Annu Rev Med 1967;18:97-112.

31. Yan, C., X. Lian, Y. Li, et al., Macrophage-specific expression of human lysosomal acid lipase corrects inflammation and pathogenic phenotypes in lal-/- mice. Am J Pathol 2006;169:916-26. 


\section{Supplemental Figure}

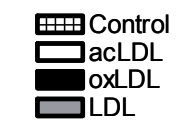

Itgam

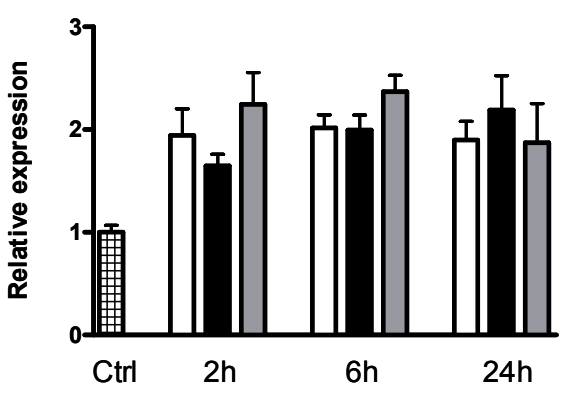

24h - Mac1 staining

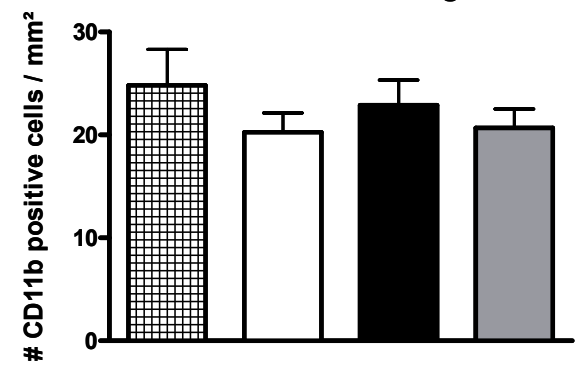

Figure S3.1 Infiltrating macrophages. Hepatic gene expression of Integrin alpha-M (Itgam) and quantification of histology for infiltrating macrophages (Mac1 staining) after $24 \mathrm{~h}$ of injection. 


\section{Chapter 4}

\section{Lysosomal cholesterol in Kupffer cells, particularly}

when oxidized, contributes to murine steatohepatitis

SMA Walenbergh, T Houben, T Hendrikx, PJ van Gorp, MLJ Jeurissen, MH Lenders, MJJ Gijbels, J Plat, MH Hofker, CJ Binder, D Lütjohann, F Verheyen, GH Koek, R Shiri-Sverdlov

Submitted 


\section{Abstract}

\section{Background and aims}

Recently, the importance of lysosomes within the metabolic syndrome, including fatty liver disease, is gaining increasing attention. It has been suggested that macrophages during atherosclerosis as well as Kupffer cells (KCs) during hepatic inflammation demonstrate properties of an acquired lysosomal storage disorder (LSD). So far, it is unclear whether there is a causal relationship between lysosomal cholesterol accumulation (LCA) in KCs and hepatic inflammation. Additionally, the specific contribution of the oxidized LDL (oxLDL) fraction to LCA, its concomitant effect on lysosomal function, and on hepatic inflammation is unexplored.

\section{Methods}

Irradiated low-density lipoprotein receptor knockout $\left(\mathrm{Ldll}^{-/}\right)$mice were transplanted (-tp) with bone marrow from mutant Niemann-pick type $\mathrm{C} 1$ (Npc1 $^{\text {mut }}$ ) mice, i.e. these mice develop LCA, and bone marrow from control wildtype (Wt) mice and fed a high-fat diet. To induce high levels of anti-oxidized low-density lipoprotein (anti-oxLDL) antibodies, $\mathrm{Npc1}^{\text {mut }}$-tp mice were immunized every two weeks with heat-inactivated pneumococci until the end of the experiment.

\section{Results}

Compared to $\mathrm{Wt}, \mathrm{NPC1}^{\text {mut }}$-tp mice displayed severe hepatic inflammation and fibrosis. Thus, LCA in KCs is a trigger for hepatic inflammation. Next, we demonstrated that by elevating the levels of anti-oxLDL antibodies, the cholesterol metabolism, lysosomal dysfunction and liver inflammation were improved.

\section{Conclusions}

This study provides evidence for a direct causal link between LCA and hepatic inflammation in $\mathrm{KCs}$ with a specific role for oxLDL. 


\section{Introduction}

Hepatic steatosis (excessive lipid accumulation), non-alcoholic steatohepatitis (NASH or hepatic inflammation) as well as irreversible fibrosis and cirrhosis, are liver conditions all referred to as non-alcoholic fatty liver disease (NAFLD). In 2011, the prevalence of NASH was estimated to be approximately $29 \%$ in a group of ultrasound positive patients of a general population. ${ }^{1}$ Since NASH sets the stage for further progress into chronic liver damage, it is important to understand the mechanisms that trigger this inflammatory response. ${ }^{2}$ Therefore, better understanding of the mechanisms that lead to hepatic inflammation is required, as this may help to identify novel treatment strategies against this disease.

In recent years it has begun to emerge that lysosomes, apart from their main function as the cell's 'suicidal bag', play a significant role in many human diseases including cancer ${ }^{3}$ and various inflammatory diseases. ${ }^{4}$ Increasing evidence from our group and others has shown that atherosclerosis and NASH demonstrate properties of an acquired lysosomal storage disorder (LSD) and share disease mechanisms. ${ }^{5-9}$ This observation was recently confirmed by the presence of cholesterol-containing Kupffer cells (KCS) in livers of NASH patients. ${ }^{10}$ Due to excessive lysosomal accumulation of cholesterol, LSDs are characterized by prominent lysosomal abnormalities, ranging from disturbed intracellular trafficking ${ }^{11}$, lysosomal enzyme deficiencies ${ }^{12}$ and changes in lysosomal $\mathrm{pH}^{13}$ Thus, it becomes evident that lysosomal abnormalities are not limited to LSDs, such as Niemann-Pick disease type C1 (NPC1) only, but can also be linked to other inflammatory diseases, including atherosclerosis and NASH.

Previous studies demonstrated a strong association between lysosomal cholesterol accumulation and inflammation. ${ }^{14-16}$ Furthermore, unlike acetylated LDL (acLDL) and native $L D L$, it is specifically the oxidized $L D L$ (oxLDL) fraction that tends to accumulate inside lysosomes of macrophages. ${ }^{8,17-19}$ So far, a direct causal relationship between lysosomal cholesterol accumulation in KCs and hepatic inflammation has never been investigated. In particular, the specific contribution of the oxLDL fraction to lysosomal function and hepatic inflammation is unexplored.

To investigate the causal relationship between lysosomal cholesterol accumulation in Kupffer cells and hepatic inflammation, we used NPC1 mutant mice as a tool to induce lysosomal cholesterol accumulation in hematopoietic cells. Bone marrow from wildtype (Wt) and NPC1 mutant ( $\mathrm{Npc1}^{\text {mut }}$ ) mice were transplanted (-tp) into $\mathrm{Ldlr}^{-/-}$ acceptor mice and were put on a high-fat, high-cholesterol (HFC) diet for 12 weeks. By comparing Wt-tp and Npc1 ${ }^{\text {mut }}$-tp mice, we demonstrated for the first time that increased lysosomal cholesterol accumulation is a trigger for lysosomal malfunction inside KCs, hepatic inflammation and fibrosis. To examine the specific contribution of oxLDL to lysosomal function and hepatic inflammation, we reduced the oxLDL levels in 
plasma. Lowering plasma oxLDL was accomplished through elevation of anti-oxLDL antibodies by injecting Npc1 ${ }^{\text {mut }}$-tp with heat-inactivated pneumococci starting four weeks before the start of the HFC diet until the end of the experiment. As a control, Wt-tp and $\mathrm{Npc}^{\text {mut }}$-tp mice were injected with vehicle. We demonstrated that by elevating the levels of anti-oxLDL antibodies, the cholesterol metabolism, lysosomal dysfunction and liver inflammation were improved. Instead of total cholesterol, these data show that it is oxLDL in particular that is significantly involved in lysosomal dysfunction, foamy macrophage appearance and hepatic inflammation. Additionally, our data suggest a shared disease mechanism between LSDs and NASH.

\section{Materials and methods}

\section{Preparation immunogen}

For immunization, the heat-inactivated R36A strain of Streptococcus pneumoniae (Birmingham, Alabama) was used, still bearing the PC headgroup epitope similar to oxLDL. Colonies of the R36A strain were harvested at mid log phase after incubation at $37^{\circ} \mathrm{C}$ on blood agar plates and transferred to Todd-Hewitt plus $0.5 \%$ yeast broth. The mid log phase is characterized by an OD value of 0.425 to 0.45 at $600 \mathrm{~nm}$. S. pneumoniae was heat-inactivated at $60^{\circ} \mathrm{C}$ for 30 minutes; afterwards no colonies of this suspension were detected on blood agar plates. For freezer stocks of strain R36A, small aliquots of $S$. pneumoniae at mid log density were harvested and suspended in Todd-Hewitt plus $80 \%$ sterile glycerol and stored at $-80^{\circ} \mathrm{C}$. 8,20

\section{Mice, bone marrow transplantation, immunization, and diet}

Niemann-Pick type $\mathrm{C}^{\mathrm{m} 1 \mathrm{~N}}$ mutant ( $\mathrm{Npc}^{\text {mut }}$ ) mice on a $\mathrm{C} 57 \mathrm{BI} / 6$ background (a kind gift from Prof. Dr. Lieberman from University of Michigan Medical School) and Ldlr ${ }^{-/}$mice were housed under standard conditions and had access to food and water ad libitum. Experiments were performed according to Dutch regulations and approved by the Committee for Animal Welfare of Maastricht University. Twenty-two week old female LdIr-/- mice were lethally irradiated and transplanted with either wildtype or Npc1 ${ }^{\text {mut }}$ bone marrow. Five weeks after bone marrow transplantation, mice were divided into three groups ( $n=11$ for each group) and the immunization protocol started whereby mice received the equivalent of $10^{8}$ colony-forming units of heat-killed R36A emulsified in $200 \mu \mathrm{l}$ sterile $0.9 \% \mathrm{NaCl}$ for the primary subcutaneous immunization. Subsequently, two intraperitoneal booster immunizations were administered every two weeks. Control groups received a $0.9 \% \mathrm{NaCl}$ injection only. After four weeks of immunization, all mice were given an HFC diet for twelve weeks, containing $21 \%$ butter and $0.2 \%$ cholesterol (diet 1635; Scientific Animal Food and Engineering, Villemoisson-sur-Orge, France). From the start of the diet, intraperitoneal booster 
immunizations were administered every three weeks. Blood from the tail vein was collected at the start of immunization and diet as well as at the end of the diet. Subsequently, mice were sacrificed. Liver tissue was isolated and snap-frozen in liquid nitrogen and stored at $-80^{\circ} \mathrm{C}$ or fixed in $4 \%$ formaldehyde/PBS.

\section{Auto-antibody titers against IgM}

Specific antibody titers against modified LDL in plasma were determined as described elsewhere. ${ }^{21-22}$ Plasma was serially diluted and antibody binding was measured by chemiluminescent enzyme-linked immunosorbent assay. A titer was defined as the reciprocal of the maximal dilution at which binding of the secondary antibody was twice as high as the background binding.

\section{Plasma lipid analysis}

Plasma cholesterol levels were measured with an enzymatic color test (cholesterol CHOD-PAP; 1489232; Roche, Basel, Switzerland) according to the manufacturer's protocols on a Benchmark 550 Micro-plate Reader (170-6750XTU; Bio-Rad, Hercules, CA).

\section{Liver lipid analysis}

Approximately $50 \mathrm{mg}$ of frozen liver tissue was homogenized for 30 seconds at $5000 \mathrm{rpm}$ in a closed tube with 1.0- $\mathrm{mm}$ glass beads and $1.0 \mathrm{ml} \mathrm{SET}$ buffer (sucrose $250 \mathrm{mmol} / \mathrm{l}$, EDTA $2 \mathrm{mmol} / \mathrm{l}$, and Tris $10 \mathrm{mmol} / \mathrm{l}$ ). Complete cell destruction was done by 2 freeze-thaw cycles and 3 times passing through a 27-gauge syringe needle and a final freeze-thaw cycle. Protein content was measured with the bicinchoninic acid (BCA) method (23225; Pierce, Rockford, IL). Liver cholesterol, triglyceride, campesterol and sitosterol levels were quantified as described previously. ${ }^{23,24}$

\section{Liver histology}

Frozen liver sections $(7 \mu \mathrm{m})$ were fixed in acetone and subsequently blocked for endogenous peroxidase by incubation with $0.25 \%$ of $0.03 \% \mathrm{H} 2 \mathrm{O} 2$ for 5 minutes. Primary antibodies used were against infiltrated macrophages and neutrophils (ratanti-mouse Mac-1 [M1/70]) neutrophils (rat-anti-mouse Ly6-C, clone NIMP-R14) (generous gift from Prof Heeringa, Groningen, The Netherlands), Kupffer cells (KCs) (rat-anti-mouse CD68, clone FA11) (generous gift from Prof Gordon, Oxford, UK). 3-Amino-9 ethylcarbazole (AEC) (A85SK-4200.S1; Bio-connect, Huissen, The Netherlands) was applied as color substrate and hematoxylin (4085.9002, Klinipath, Duiven, The Netherlands) for nuclear counterstaining. Sections were enclosed with Faramount aqueous mounting medium (S302580; DAKO, Glostrup, Denmark). For the lipid staining, Oil Red O (00625; Sigma-Aldrich) was used. 
Paraffin-embedded liver sections $(4 \mu \mathrm{m})$ were stained with hematoxylin-eosin (HE; Hematoxilin, 4085.9002; Klinipath, Duiven, The Netherlands; and Eosin, E4382; SigmaAldrich) and Sirius red (Direct Red 80, 43665; Sigma-Aldrich). Pictures were taken with a Nikon digital camera DMX1200 and ACT-1 v2.63 software (Nikon Instruments Europe, Amstelveen, The Netherlands). Immune cells were counted in 6 microscopical views (original magnification, $200 \times$ ) and were noted as cells/square millimeter.

\section{Acid phosphatase activity assay}

Hepatic acid phosphatase activity was determined by the acid phosphatase assay kit (10008051, Cayman Chemical Company, USA). Liver homogenates were diluted 20 times in assay buffer, and transferred to a 96-well plate containing $20 \mu \mathrm{l}$ assay buffer. Next, $20 \mu \mathrm{l}$ AP substrate solution was added to each well to initiate the reaction. After 20 minutes incubation at $37^{\circ} \mathrm{C}$, the reaction was stopped by adding $100 \mu$ l of stop solution to all wells. The absorbance was measured using a Bio-Rad Benchmark 550 Micro-plate reader at 405-414 nm (Bio-Rad, Hercules, CA, USA). The activity was calculated as $\mu \mathrm{mol} / \mathrm{min} / \mathrm{mol} ; 1$ unit corresponds with the amount of acid phosphatase required to release $1 \mu \mathrm{mol}$ of phosphatase per minute at $37^{\circ} \mathrm{C}$.

\section{Electron microscopy}

Livers were freshly isolated from the mice and perfused and fixed overnight with $2.5 \%$ glutaraldehyde (Ted Pella, Redding, CA, USA). Tissue fragments were washed and post fixed in $1 \%$ osmium tetroxide. Tissues were subsequently dehydrated through $100 \%$ ethanol, cleared with propylene oxide, and embedded in epoxy resin. Sections of $1 \mu \mathrm{m}$ were stained with toluidine blue to identify the presence of foamy KCs. Next, sections of 70-90 nm were cut on an ultra-microtome, mounted on Formvar-coated (1595E, Merck) 75 mesh copper grids and counterstained with uranyl acetate and lead citrate before analysis on a Philips CM100 transmission electron microscope.

\section{RNA isolation and quantitative polymerase chain reaction}

Total RNA was isolated from approximately $25 \mathrm{mg}$ of mouse liver tissues as described previously. ${ }^{25}$ All applications were performed according to the manufacturers' protocols. Total RNA (500 ng) from each individual mouse was converted into firststrand complementary DNA with the iScript cDNA synthesis kit (170-8891; Bio-Rad, Hercules, CA) according to the manufacturer's instructions. The changes in gene expression of inflammatory markers were determined by quantitative polymerase chain reaction (qPCR) on an SDS $7900 \mathrm{HT}$ by using PowerSybr Green mastermix (4329001 and 4368708; both Applied Biosystems, Foster City, CA) and 10 ng of cDNA. For each gene, a standard curve was generated with a serial dilution of a liver CDNA pool. To standardize for the amount of cDNA, Cyclophillin A was used as the reference gene. Primer sets for the selected genes were developed with Primer Express 
version 2.0 (Applied Biosystems) using default settings. Data from qPCR were analyzed according to the relative standard curve method.

\section{Bone marrow-derived macrophages}

Bone marrow-derived macrophages (BMM) were isolated from the tibiae and femurs of NPC $1^{\text {mut }}$ mice. Cells were cultured in RPMI-1640 (GIBCO Invitrogen, Breda, the Netherlands) with $10 \%$ heat-inactivated fetal calf serum (Bodinco B.V. Alkmaar, the Netherlands), penicillin $(100 \mathrm{U} / \mathrm{ml})$, streptomycin $(100 \mu \mathrm{g} / \mathrm{ml})$ and L-glutamine $2 \mathrm{mM}$ (all GIBCO Invitrogen, Breda, the Netherlands), supplemented with 20\% L929conditioned medium (LCM) for 8-9 days to generate BMM. After attachment, macrophages were seeded at 350,000 cells per well in 24-well plates and incubated for 24h with oxLDL ( $25 \mathrm{\mu g} / \mathrm{ml}$; Alfa Aesar: J65591, Wardhill, MA, USA), with or without anti-oxLDL EO6 antibodies (Avanti Polar Lipids, Alabaster, AL, USA). Then cells were washed and stimulated with LPS $(100 \mathrm{ng} / \mathrm{ml})$ for $4 \mathrm{~h}$. Finally, supernatant was collected for protein measurements and cells were lysed for mRNA expression analysis.

\section{Mouse tumor necrosis factor enzyme-linked immunosorbent assay (ELISA)}

The mouse TNF ELISA assay was performed on diluted supernatant from stimulated BMM (1:10) according to the manufacturer's instructions (88-7324-88; eBioscience, San Diego, CA, USA).

\section{Statistical analysis}

Data was statistically analyzed by performing two-tailed non-paired $t$-tests using GraphPad Prism, version 4.03 for Windows. Data were expressed as the mean \pm SEM and considered significant at $\mathrm{p}<0.05{ }^{*},{ }^{* *}$ and $* * *$ indicate $\mathrm{p}<0.05,0.01$ and 0.001 resp.

\section{Results}

\section{Compared to Wt-tp mice, bone marrow-tp Npc1 ${ }^{\text {mut }}$ mice show a severe structural phenotype in the liver}

Due to malfunction of the NPC1 protein, excessive amounts of cholesterol are trapped inside lysosomes causing the formation of extremely large lysosomes. Manifestations of the NPC1 phenotype include cognitive impairments and hepatosplenomegaly. ${ }^{26}$ Indeed, liver and spleen weights were dramatically increased in mice transplanted with $\mathrm{Npc1}{ }^{\text {mut }}$ bone marrow compared to Wt-tp mice, while body weights were reduced (Supplementary Figures S4.1A-C). Relative spleen weight was slightly but not 
significantly decreased in immunized Npc1 ${ }^{\text {mut }}$-tp compared to Npc1 ${ }^{\text {mut }}$-tp that did not receive immunization.

To confirm whether the bone marrow transplantation was successful, we performed electron microscopy on livers from Wt-tp and $\mathrm{Npc} 1^{\text {mut }}$-tp mice. As pointed out by the lower magnification, livers from Npc1 ${ }^{\text {mut }}$-tp mice clearly demonstrated the presence of extremely large macrophage granulomas in contrast to Wt-tp mice (Figure 4.1A). These electron microscopy images were confirmed by a hematoxylin and eosin staining (Supplementary Figure S4.1D). The granuloma macrophages showed severe accumulation of vacuolar structures containing a huge amount of small membraneous inclusions. While focal vesicular dilations of cristae in the mitochondria of the single macrophages were evident at higher magnifications, indicating that these cells are the resident $\mathrm{KCs}$, no such structures were observed within the mitochondria of the large macrophage granulomas (pictures not shown). Thus, these characteristics indicate that the latter cells can be denominated as macrophages derived from bone marrow and consist of abnormally swollen macrophages that are typical for an NPC1 phenotype. Additionally, in the Npc1 ${ }^{\text {mut }}$-tp group, the majority of resident KCs were located adjacent to the large macrophage granulomas, and similarly, also contained various numbers of small lipid inclusions. No detectable differences in phenotype between non-immunized and immunized $\mathrm{Npc}^{\text {mut }}{ }^{-\mathrm{tp}}$ mice were seen.

\section{Increased IgM autoantibody titers against oxLDL after immunization with heat-inactivated pneumococci}

To determine whether IgM anti-oxLDL antibodies are beneficial for lysosomal cholesterol accumulation and hepatic inflammation, $\mathrm{Npc}{ }^{\text {mut }}$-tp mice received an HFC diet and were immunized with heat-inactivated pneumococci. Immunization with heat-inactivated pneumococci resulted in a strong increase of plasma IgM antibodies of the EO6/T15 idiotype (Figure 4.1B), which bind oxLDL by specifically recognizing the phosphorylcholine epitope. ${ }^{27}$ Indeed, increased IgM antibodies against copperoxidized (CUOx)LDL were detected in immunized mice compared to control mice (Figure 4.1C). Thus, immunization with heat-inactivated pneumococci is able to induce a strong anti-oxLDL IgM antibody production in $\mathrm{Npc1}{ }^{\text {mut }}$-tp mice. 


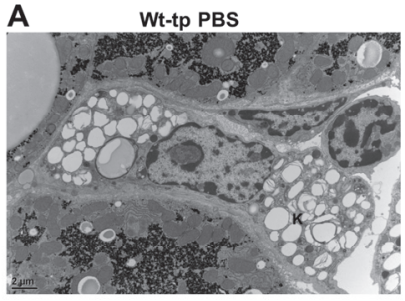

B

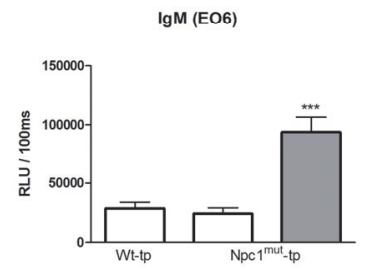

Npc1mut-tp PBS

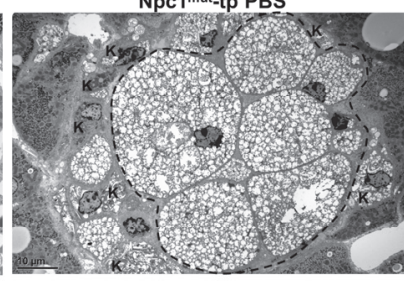

Npc1mut_tp R36A

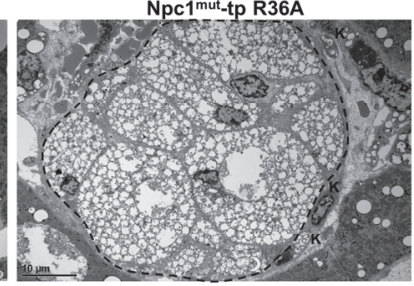

C

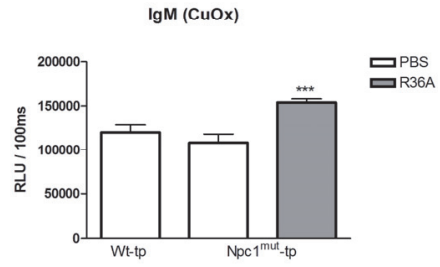

Figure 4.1 Liver phenotype of Wt-tp and $\mathrm{Npc1}^{\text {mut }}$-tp mice and IgM autoantibody titers in plasma. (A) Representative electron microscopy pictures of $\mathrm{KCS}$ and $\mathrm{Npc1}{ }^{\text {mut }}$ macrophages of Wt-tp $(2 \mu \mathrm{m})$ and (non-)immunized Npc1 ${ }^{\text {mut }}$-tp mice $(10 \mu \mathrm{m})$. Area within the dashed line: Npc1 ${ }^{\text {mut }}$ granuloma; K: Kupffer cell. IgM EO6 antibodies (B) and IgM antibodies against copper-oxidized (CUOx)LDL (C) were measured in plasma of mice with or without immunization at a dilution of 1:100. Data are expressed as relative light units (RLU) / 100ms and were triplicate determinations. Asterisks indicate significant difference from non-immunized Wt-tp and Npc1 ${ }^{\text {mut }}$-tp mice. ${ }^{* * *} \mathrm{p}<0.001$. PBS: phosphate buffered saline; R36A: heat-inactivated pneumococci.

\section{Decreased hepatic cholesterol content and foamy macrophage appearance after anti-oxLDL immunization}

To determine the overall cholesterol metabolism, we examined cholesterol levels in liver and plasma. After 12 weeks on an HFC diet, liver cholesterol was significantly elevated in Npc1 ${ }^{\text {mut }}$-tp mice when compared to Wt-tp mice. Interestingly, the hepatic cholesterol content was decreased after immunization of $\mathrm{Npc}^{\text {mut }}{ }^{\text {-tp }}$ mice compared

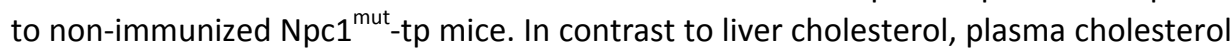
was dramatically reduced by almost $50 \%$ in $\mathrm{Npc}^{\text {mut }}$-tp mice compared to Wt-tp and did not differ between immunized and non-immunized Npc1 ${ }^{\text {mut }}$-tp mice (Figure 4.2A). In line with hepatic cholesterol content, hematopoietic mutation of NPC1 increased the hepatic phytosterol levels, campesterol and sitosterol, while these levels were lowered after administration of heat-inactivated pneumococci. To note, within the brain, these plant sterols were unaffected (Supplementary Figures S4.2A-D). Additionally, we compared the hepatic gene expression of the cholesterol efflux transporters $A B C A 1$ and $A B C G 1$. While $A b c a 1$ gene expression is comparable between all the groups, $A b c g 1$ is increased in the $\mathrm{Npc1}^{\text {mut }}$-tp mice (Figure 4.2B). Upon antioxLDL immunization, $A b c g 1$ gene expression is significantly reduced. To investigate the foamy appearance of infiltrated macrophages within the liver, tissue sections 
were stained for CD68, a macrophage marker (Figure 4.2C-D). Quantification of the CD68 positive area revealed a dramatic increase in size of foamy macrophages in $\mathrm{Npc1}^{\text {mut }}$-tp mice compared to Wt-tp mice (Figure 4.2C). After immunization with heatinactivated pneumococci, Npc1 ${ }^{\text {mut }}$-tp mice displayed liver macrophages with a significant lower foamy appearance compared to non-immunized mice. Opposite to liver cholesterol, liver triglyceride levels were decreased in $\mathrm{Npc} 1^{\text {mut }}$-tp mice compared to Wt-tp mice and remained similar between immunized and non-immunized $\mathrm{Npc1}^{\text {mut }}$-tp mice (Figure 4.2E). An Oil Red O staining confirmed the biochemical liver triglyceride measurements (Figure 4.2F).

A

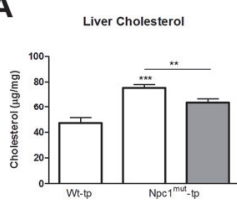

C

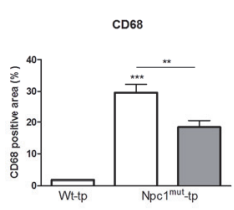

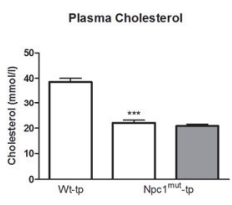

D

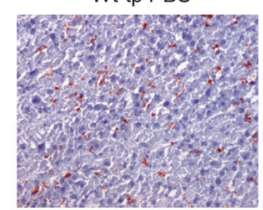

B

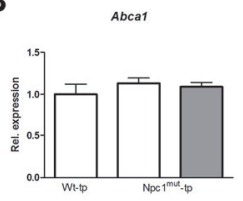

Npc1 $1^{\text {mut-Lp PBS }}$

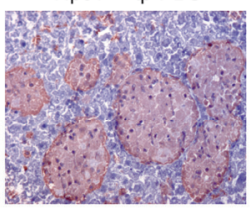

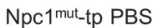

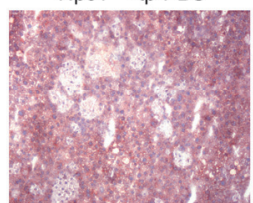

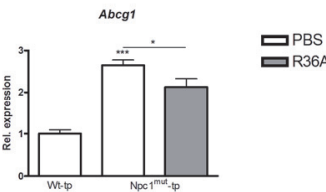

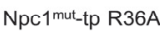

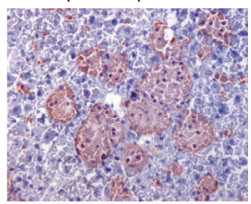

Npc1mut_tp R36A

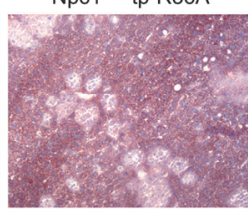

Figure 4.2 Cholesterol parameters. Cholesterol levels in liver as well as in plasma (A) of Wt-tp, (non-)immunized Npc1 ${ }^{\text {mut }}$-tp mice on an HFC diet. Hepatic gene expression analysis of the cholesterol efflux transporters $A b c a 1$ and $A b c g 1$ (B). Quantification of the CD68 positive area (C) by using Adobe Photoshop v. 9.0. Representative histological pictures of the CD68 staining (magnification $\times 200$ ) of macrophages of Wt-tp and (non-)immunized Npc1 ${ }^{\text {mut }}$-tp mice after a 12 week HFC diet (D). Liver triglyceride levels (E) and representative histological images of the Oil Red $\mathrm{O}$ staining (F). Asterisks indicate significant difference from Wt-tp mice. ${ }^{*},{ }^{* *}$ and $* * *$ indicate $p<0.05,0.01$ and 0.001 resp. PBS: phosphate buffered saline; R36A: heat-inactivated pneumococci.

\section{Livers of non-immunized Npc1 ${ }^{\text {mut }}$-tp mice show defects in lysosomal (enzyme) function compared to Wt-tp mice, but improved upon anti- oxLDL immunization}

NPC1 disease is associated with disturbed levels of lysosomal enzymes including cathepsins. ${ }^{15,28}$ To determine the lysosomal enzyme status in the liver, the activity of the lysosomal enzyme acid phosphatase was measured. Hepatic acid phosphatase activity of non-immunized $\mathrm{Npc1}{ }^{\text {mut }}$-tp mice was significantly higher than in Wt-tp 
mice, while immunization normalized acid phosphatase activity in Npc1 ${ }^{\text {mut }}$-tp mice (Figure 4.3A). Similar to acid phosphatase, gene expression levels of the lysosomal enzymes Cathepsin $D, S$ and $K$ were increased in non-immunized $N p c 1^{\text {mut }}$-tp mice compared to Wt-tp mice. Upon immunization, these lysosomal enzymes were significantly reduced (Figure 4.3B-D). To test whether intracellular cholesterol trafficking is affected in this study, we analyzed gene expression of the intracellular cholesterol transporters NPC1 and NPC2. In total liver, Npc1 gene expression is similar in all groups, while $N p c 2$ is elevated in $N p c 1^{\text {mut }}$-tp mice compared to Wt-tp mice (Figure 4.3E-F). In comparison to non-immunized Npc1 ${ }^{\text {mut }}$-tp mice, Npc2 hepatic gene expression is significantly reduced after immunization.

A

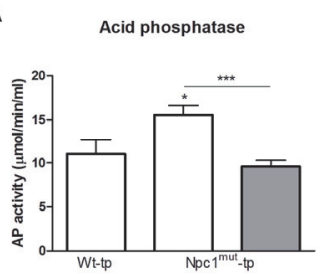

D

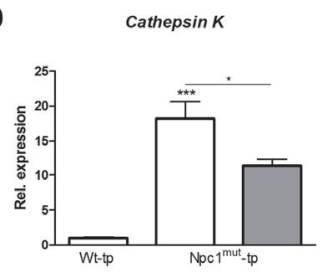

B

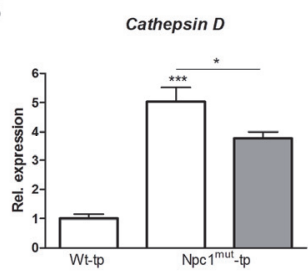

$\mathbf{E}$

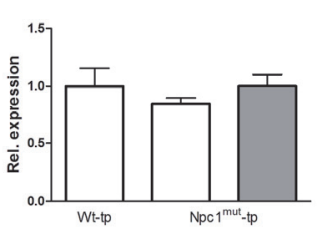

C

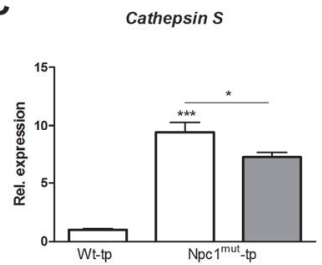

$\mathbf{F}$

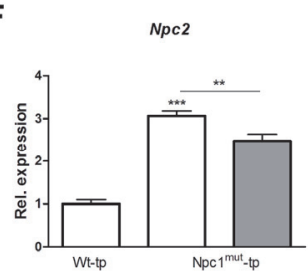

Figure 4.3 Parameters of lysosomal (enzyme) dysfunction. (A) Hepatic activity of the lysosomal enzyme, acid phosphatase, after feeding Wt-tp and Npc1 ${ }^{\text {mut }}$-tp mice a 12-week HFC diet. Hepatic gene expression of the lysosomal enzymes Cathepsin $D, S$ and $K$ (B-D) and the intracellular cholesterol transporters $N p c 1$ and $N p c 2$ (E-F). Gene expression data are set relative to Wt-tp mice. Asterisks indicate significant difference from Wt-tp mice. ${ }^{*}, * *$ and $* * *$ indicate $p<0.05$, 0.01 and 0.001 resp. PBS: phosphate buffered saline; R36A: heat-inactivated pneumococci. Npc1: Niemann-Pick Type C1; Npc2: Niemann-Pick Type C2.

\section{The dramatic increase of hepatic inflammation in Npc1 ${ }^{\text {mut }}$-tp mice is lowered upon pneumococcal immunization}

To assess hepatic inflammation, liver sections were stained for the inflammatory markers Mac-1 (infiltrated macrophages and neutrophils) and NIMP (neutrophils). These inflammatory markers demonstrated a significant higher level of inflammation in livers of non-immunized $N p c 1^{\text {mut }}$-tp mice than Wt-tp mice (Figure 4.4A). After immunization, hepatic inflammation was considerably decreased in $\mathrm{Npc1}{ }^{\text {mut }}$-tp mice compared to control-injected Npc1 ${ }^{\text {mut }}$-tp mice. Representative histological pictures of the Mac-1 staining for all three groups are shown in Figure 4.4B. Here, single 
macrophages were stained positive for Mac-1, while the large macrophage granulomas in the $\mathrm{Npc1}^{\mathrm{mut}}$-tp group are Mac-1 negative.

A

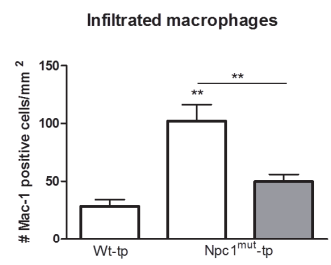

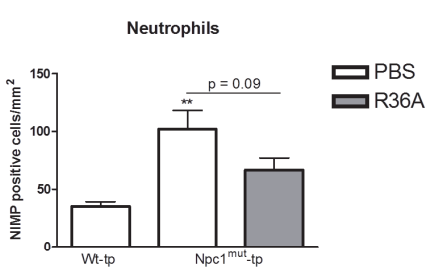

B
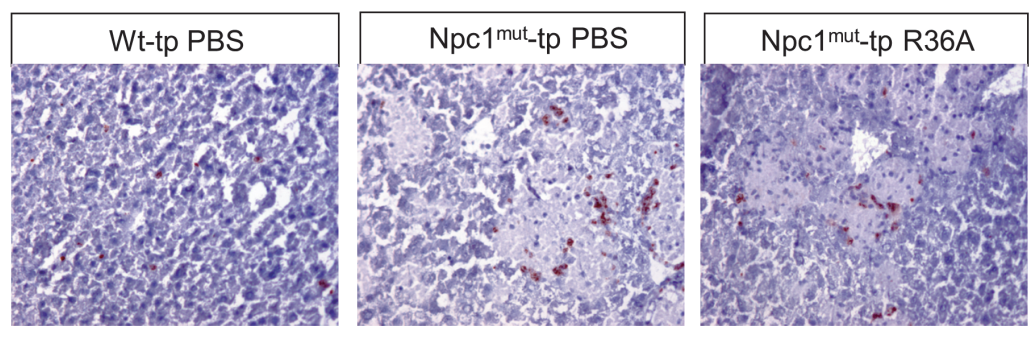

C
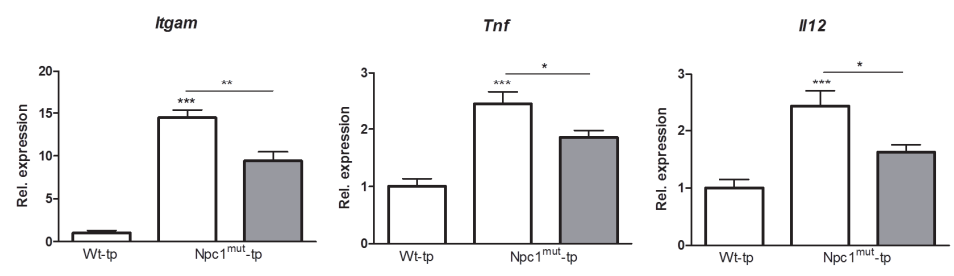

Caspase-1

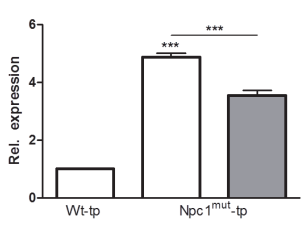

Cxcr4

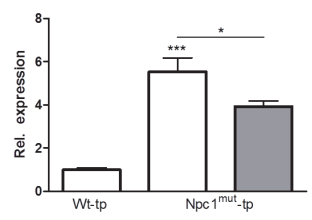

ccr2

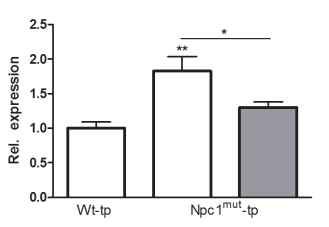

Figure 4.4 Parameters of hepatic inflammation. (A) Liver sections were stained for both macrophages and neutrophils (Mac-1) and neutrophils solely (NIMP), and were counted. (B) Representative images of the Mac-1 staining (magnification $\times 200$ ) after feeding Wt-tp and (non-)immunized Npc1 ${ }^{\text {mut }}$-tp mice an HFC diet for 12 weeks. (C) Hepatic gene expression analysis for Itgam, Tnf, II12, Caspase-1, Cxcr4 and Ccr2. Gene expression data were set relative to Wt-tp mice. Asterisks indicate significant difference from Wt-tp mice. ${ }^{*} p<0.05 ;{ }^{* *} p<0.01 ;{ }^{* * *} p<0.001$. PBS: phosphate buffered saline; R36A: heat-inactivated pneumococci; Itgam: integrin alpha M; Tnf: tumor necrosis factor; II-12: interleukin-12; CXcr4: CXC chemokine receptor-4; Ccr2: CC chemokine receptor-2. 
To confirm the histological data, gene expression analysis of the liver was performed. As shown in Figure 4.4C, inflammatory genes, integrin alpha M (Itgam), tumor necrosis factor (Tnf), interleukin 12 (I/12), Caspase-1, CXC chemokine receptor-4 (Cxcr4) and CC chemokine receptor-2 (Ccr2) were significantly increased in nonimmunized $\mathrm{Npc}^{\mathrm{mut}}$-tp mice compared to Wt-tp mice. After immunization, these inflammatory-related genes decreased substantially compared to Npc1 ${ }^{\text {mut }}$-tp mice without immunization. To further investigate the direct effect of anti-oxLDL antibodies on inflammation, we isolated $\mathrm{NPC} 1^{\text {mut }}$ bone marrow-derived macrophages (BMM) and stimulated these with oxLDL, with or without the anti-oxLDL (EO6) antibodies. These EO6 antibodies are identical to the anti-oxLDL antibodies that are induced in vivo upon the immunization protocol. $^{29}$ In the presence of the EO6 antibodies, NPC1 mutant BMM demonstrated significantly lower TNF protein levels, which was confirmed by reduced mRNA levels of $\operatorname{Tnf}$ and $\mathrm{Ccr} 2$, compared to incubation without the EO6 antibodies (Supplementary Figures S4.3A-B). Thus, we found that BMM derived from NPC1 mutant mice, which are incubated with oxLDL, are less inflammatory in the presence of anti-oxLDL antibodies.

\section{$\mathrm{Npc1}^{\text {mut }}$-tp mice display increased hepatic fibrosis compared to Wt-tp mice}

To evaluate the degree of liver fibrosis, an advanced stage of NASH, a collagen staining (Sirius Red) was performed. Hepatic collagen levels were significantly higher in non-immunized Npc1 ${ }^{\text {mut }}$-tp mice compared to Wt-tp mice (Figure 4.5A), which is probably due to the fact that there is collagen present around the large macrophage granulomas (Figure 4.5B). Immunization of $\mathrm{Npc1}^{\text {mut }}$-tp mice showed a trend towards a decrease in the level of fibrosis compared to $\mathrm{Npc1}{ }^{\text {mut }}$-tp mice without immunization (Figure 4.5A). In line with these findings, hepatic gene expression levels of the fibrotic markers, transforming growth factor beta $(T g f-B)$ and tissue inhibitor of metalloproteinase-3 (Timp3) were significantly higher in non-immunized Npc1 ${ }^{\text {mut }}$-tp mice than in Wt-tp mice (Figure 4.5C). After immunization, these fibrotic markers were lower compared to non-immunized $\mathrm{Npc}^{\text {mut }}$-tp mice. Altogether, these data indicate that $\mathrm{Npc}^{\text {mut }}{ }^{-t p}$ mice are more susceptible for liver fibrosis and emphasize a potential contribution of oxLDL to more advanced stages of NASH. 
A

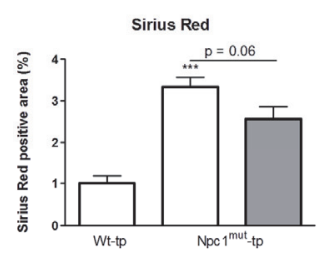

B

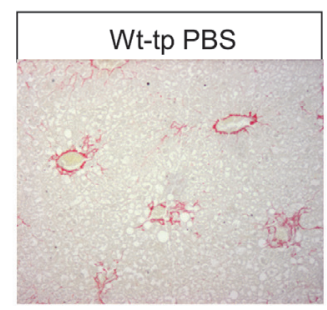

C
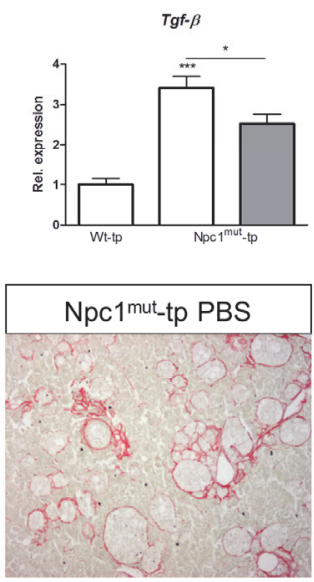

ㅁBS R36A
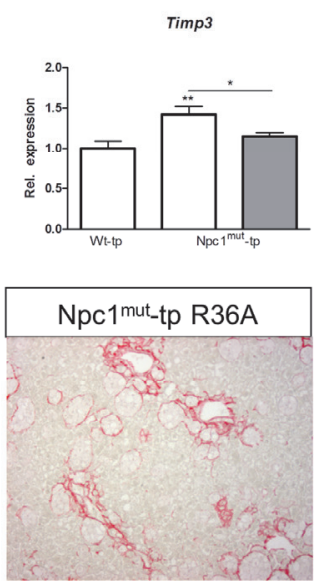

Figure 4.5 Parameters of liver fibrosis. (A) Quantification of Sirius Red (collagen) staining after 12 weeks of an HFC diet. Livers were quantified by using Adobe Photoshop v. 9.0. (B) Representative pictures of Sirius Red staining (original magnification, 100x) of Wt-tp mice and Npc1 ${ }^{\text {mut }}$-tp mice with or without immunization on an HFC diet for 3 months. (C) Gene expression analysis of the fibrosis markers, Tgf- $B$ and Timp3. Gene expression data are shown relative to Wt-tp mice. *: $\mathrm{p}<0.05$; ***: $\mathrm{p}<0.001$. PBS: phosphate buffered saline; R36A: heat-inactivated pneumococci; Tgf-6: transforming growth factor-beta; Timp3: tissue inhibitor of metalloproteinase-3.

\section{Discussion}

So far, the causal relationship between lysosomal cholesterol accumulation in KCs and hepatic inflammation has not been investigated. Additionally, the specific contribution of the accumulated oxLDL to lysosomal dysfunction and hepatic inflammation is unclear. As a tool to induce lysosomal cholesterol accumulation in KCs, we transplanted NPC1 mutant bone marrow into hyperlipidemic mice. Npc1 ${ }^{\text {mut }}$-tp mice elicited a dramatic increase in hepatic inflammation and fibrosis compared to Wt-tp mice. Thus, lysosomal cholesterol accumulation in KCs is a trigger for hepatic inflammation and suggests the existence of a shared mechanism between hepatic inflammation and LSDs. Moreover, our data further provide evidence that oxLDL accumulation in the KCs is likely to be more harmful than native LDL.

Previous studies by us and others demonstrated merely an association between lysosomal cholesterol accumulation and hepatic inflammation. ${ }^{8,9,16,19,30}$ Additional evidence shows that also other inflammatory diseases, such as atherosclerosis, feature lysosomal cholesterol accumulation in macrophages. ${ }^{31,32}$ NPC1 is a fatal autosomal recessive disorder that causes excessive lysosomal storage of cholesterol 
with concomitant lysosomal defects due to mutations in the NPC1 gene. ${ }^{33}$ Whole body deletion of NPC1 in mice shows a progressive course of life, with weight loss, impaired cognitive function and early death (8-12 weeks of age). ${ }^{34}$ To induce lysosomal cholesterol accumulation in the hematopoietic system, we therefore choose to transplant NPC1 mutant bone marrow into hyperlipidemic mice as a tool to investigate the causal relationship between lysosomal cholesterol accumulation inside KCs and hepatic inflammation. Despite the fact that NPC1 deficiency was only present in the hematopoietic system in our study, we observed severe hepatic inflammation and fibrosis. Thus, lysosomal cholesterol accumulation inside $\mathrm{KCs}$ is the main driver for hepatic inflammation. Additionally, our data point towards the overlapping disease mechanism between hepatic inflammation and LSDs.

Correct functioning of the lysosomes is tightly regulated and includes many lysosomal parameters, i.e. lysosomal pH, lysosomal enzyme trafficking, number and size. A slight change in one of these parameters can cause lysosomal dysfunction. As a result, a strong association exists between lysosomal cholesterol accumulation and a disturbance in lysosomal enzymes. Previous data displayed modified levels of cathepsin D, a lysosomal enzyme, in mice with NPC brain pathology ${ }^{35}$ and in plasma of pediatric NASH patients. ${ }^{36}$ In line with these data, we now demonstrate increased hepatic gene expression levels of several cathepsins and acid phosphatase activity in Npc1 ${ }^{\text {mut }}$-tp mice, hereby strongly implicating lysosomal dysfunction. Altogether, we now demonstrate for the first time a direct causal relationship between lysosomal cholesterol accumulation, concomitant lysosomal dysfunction, in KCs and hepatic inflammation.

NPC1 disease is characterized by a dramatic cholesterol imbalance with an important contribution for the liver. Since the liver plays a unique role in the cholesterol metabolism, it is therefore one of the most affected tissues during NPC1 disease. ${ }^{37}$ As expected, whole liver cholesterol and the foamy KC appearance were dramatically increased in Npc1 ${ }^{\text {mut }}$-tp mice compared to Wt-tp mice, while plasma cholesterol levels were reduced. These data clearly show that the cholesterol is trapped inside the liver cells and cannot be transported into the plasma. The concomitant increase in hepatic cholesterol efflux transporters, ABCG1 and NPC2, could be attributed to a potential protective mechanism as the liver vigorously attempts to lower the extremely high cholesterol levels. NPC2 is known to bind sterols in a direct way, ${ }^{38}$ therefore, in line with an increase in NPC2 gene expression in the livers of $\mathrm{Npc}^{\text {mut }}{ }_{\text {-tp }}$ mice, we found elevated hepatic levels of the plant sterols, campesterol and sitosterol. ${ }^{39}$ In contrast to cholesterol, hepatic triglyceride content was decreased in Npc1 ${ }^{\text {mut }}$-tp mice compared to Wt-tp mice, which is in line with data published by Uronen et al. ${ }^{40}$ Interestingly, the anti-oxLDL immunization did not affect hepatic triglyceride levels. These findings suggest that, unlike triglycerides, the anti-oxLDL immunization plays a significant role in cholesterol metabolism in the liver. 
Whether modified or unmodified cholesterol triggers hepatic inflammation is unknown. Previously, an important role for oxLDL in inflammation has been described. ${ }^{29,41}$ In addition, our group and others recently demonstrated a relationship between inflammation and accumulation of oxLDL inside lysosomes of macrophages. ${ }^{8,19,32,42}$ In the circulation of healthy individuals the amount of minimally modified oxLDL is very small and has been reported to represent only $0.001 \%$ of native LDL. ${ }^{43}$ However, the oxLDL fraction can increase to approximately $0.6-1.8 \%$ in patients with the metabolic syndrome, although the percentage of oxLDL is still considerably low. ${ }^{44}$ Similarly, cholesterol oxidation products in plasma were increased in human NPC1 patients compared to age-matched control subjects. ${ }^{45}$ In NASH patients, it has been found that plasma oxLDL is only 1.4 times higher than the average oxLDL level detected in control subjects. ${ }^{46}$ Therefore, the improvements in liver and lysosomes after preventing oxLDL uptake by pneumococcal immunization exceeded our expectations and point towards the important contribution of oxLDL in hepatic inflammation. Apart from oxLDL, other modified lipids such as aggregated LDL (aggLDL) and cholesteryl-ester rich lipid dispersions (DISP) may also lead to cholesterol accumulation inside lysosomes and sustained inhibition of hydrolysis and subsequent efflux. $^{31,47}$ In addition, sphingomyelin-rich particles were shown to be highly present inside lysosomes of macrophages after incubation with different lipoprotein species. ${ }^{48}$ To note, these lipids (i.e. aggLDL, DISP and sphingomyelin) were not studied in these experiments and could provide an explanation for the fact that hepatic inflammation in immunized NPC1 ${ }^{\text {mut }}$-tp mice was not completely abolished to control values.

Current treatment options against hepatic inflammation are limited. Previous in vivo studies in $\mathrm{Ldlr}^{-/}$mice have already shown that pneumococcal vaccination is useful to reduce inflammatory disorders such as NASH and atherosclerosis., ${ }^{8,29}$ Other approaches to reduce plasma oxLDL, via passive immunization ${ }^{43}$ or immunization against the main epitope of oxLDL, phosphorylcholine, all demonstrated to improve inflammation. ${ }^{50}$ Thus, these data suggest that lowering oxLDL levels are beneficial for inflammation. Our data confirm that immunization with heat-inactivated pneumococci could be useful to reduce hepatic inflammation and other metabolic diseases underlying lysosomal cholesterol accumulation.

\section{Conclusions}

In conclusion, we demonstrate for the first time that increased lysosomal cholesterol accumulation in KCs causes hepatic inflammation. Rather than total cholesterol inside lysosomes, we specifically show that oxLDL significantly contributes to lysosomal dysfunction, cholesterol homeostasis and the hepatic inflammatory response. An oxLDL-targeted immunization protocol could represent a therapeutic strategy against hepatic inflammation and to other metabolic diseases underlying lysosomal cholesterol accumulation. 


\section{References}

1. Williams CD, Stengel J, Asike MI, et al. Prevalence of nonalcoholic fatty liver disease and nonalcoholic steatohepatitis among a largely middle-aged population utilizing ultrasound and liver biopsy: a prospective study. Gastroenterology 2011;140:124-31.

2. Kopec KL, Burns D. Nonalcoholic fatty liver disease: a review of the spectrum of disease, diagnosis, and therapy. Nutr Clin Pract 2011;26:565-76.

3. Saftig P, Sandhoff K. Cancer: Killing from the inside. Nature 2013;502:312-3.

4. Bordon Y. Immune regulation: Iysosomes at the heart of inflammation. Nat Rev Immunol 2011; 11:502.

5. Jerome WG. Advanced atherosclerotic foam cell formation has features of an acquired lysosomal storage disorder. Rejuvenation Res 2006;9:245-55.

6. Bieghs V, Rensen PC, Hofker MH, et al. NASH and atherosclerosis are two aspects of a shared disease: central role for macrophages. Atherosclerosis 2012;220:287-93.

7. Bieghs V, Hendrikx T, van Gorp PJ, et al. The cholesterol derivative 27-hydroxycholesterol reduces steatohepatitis in mice. Gastroenterology 2013;144:167-178 e1.

8. Bieghs V, van Gorp PJ, Walenbergh SM, et al. Specific immunization strategies against oxidized lowdensity lipoprotein: a novel way to reduce nonalcoholic steatohepatitis in mice. Hepatology 2012; 56:894-903.

9. Bieghs V, Verheyen F, van Gorp PJ, et al. Internalization of modified lipids by CD36 and SR-A leads to hepatic inflammation and lysosomal cholesterol storage in Kupffer cells. PLoS One 2012;7:e34378.

10. Ioannou GN, Haigh WG, Thorning D, et al. Hepatic cholesterol crystals and crown-like structures distinguish NASH from simple steatosis. J Lipid Res 2013;54:1326-34.

11. Shen D, Wang X, Li X, et al. Lipid storage disorders block lysosomal trafficking by inhibiting a TRP channel and lysosomal calcium release. Nat Commun 2012;3:731.

12. Filocamo M, Morrone A. Lysosomal storage disorders: molecular basis and laboratory testing. Hum Genomics 2011;5:156-69.

13. Cox BE, Griffin EE, Ullery JC, et al. Effects of cellular cholesterol loading on macrophage foam cell lysosome acidification. J Lipid Res 2007;48:1012-21.

14. Bieghs V, Van Gorp PJ, Wouters K, et al. LDL receptor knock-out mice are a physiological model particularly vulnerable to study the onset of inflammation in non-alcoholic fatty liver disease. PLoS One 2012; 7:e30668.

15. Liao G, Yao Y, Liu J, et al. Cholesterol accumulation is associated with lysosomal dysfunction and autophagic stress in Npc1 -/- mouse brain. Am J Pathol 2007;171:962-75.

16. Yan C, Lian X, Li Y, et al. Macrophage-specific expression of human lysosomal acid lipase corrects inflammation and pathogenic phenotypes in lal-/- mice. Am J Pathol 2006;169:916-26.

17. Jerome WG, Cash C, Webber R, et al. Lysosomal lipid accumulation from oxidized low density lipoprotein is correlated with hypertrophy of the Golgi apparatus and trans-Golgi network. J Lipid Res 1998;39:1362-71.

18. Yancey PG, Jerome WG. Lysosomal cholesterol derived from mildly oxidized low density lipoprotein is resistant to efflux. J Lipid Res 2001;42:317-27.

19. Bieghs V, Walenbergh SM, Hendrikx T, et al. Trapping of oxidized LDL in lysosomes of Kupffer cells is a trigger for hepatic inflammation. Liver Int 2013;33:1056-61.

20. Briles DE, Forman C, Hudak S, et al. Anti-phosphorylcholine antibodies of the T15 idiotype are optimally protective against Streptococcus pneumoniae. J Exp Med 1982;156:1177-85.

21. Horkko S, Bird DA, Miller E, et al. Monoclonal autoantibodies specific for oxidized phospholipids or oxidized phospholipid-protein adducts inhibit macrophage uptake of oxidized low-density lipoproteins. J Clin Invest 1999;103:117-28.

22. Binder CJ, Hartvigsen K, Chang MK, et al. IL-5 links adaptive and natural immunity specific for epitopes of oxidized LDL and protects from atherosclerosis. J Clin Invest 2004;114:427-37.

23. Lutjohann D, Stroick M, Bertsch T, et al. High doses of simvastatin, pravastatin, and cholesterol reduce brain cholesterol synthesis in guinea pigs. Steroids 2004;69:431-8.

24. Bieghs V, Wouters K, van Gorp PJ, et al. Role of scavenger receptor A and CD36 in diet-induced nonalcoholic steatohepatitis in hyperlipidemic mice. Gastroenterology 2010;138:2477-86, 2486 e1-3. 
25. Shiri-Sverdlov R, Wouters K, van Gorp PJ, et al. Early diet-induced non-alcoholic steatohepatitis in APOE2 knock-in mice and its prevention by fibrates. J Hepatol 2006;44:732-41.

26. Chang TY, Reid PC, Sugii S, et al. Niemann-Pick type $C$ disease and intracellular cholesterol trafficking. J Biol Chem 2005;280:20917-20.

27. Shaw PX, Horkko S, Chang MK, et al. Natural antibodies with the T15 idiotype may act in atherosclerosis, apoptotic clearance, and protective immunity. J Clin Invest 2000;105:1731-40.

28. Xie X, Brown MS, Shelton JM, et al. Amino acid substitution in NPC1 that abolishes cholesterol binding reproduces phenotype of complete NPC1 deficiency in mice. Proc Natl Acad Sci U S A 2011;108:15330-5.

29. Binder CJ, Horkko S, Dewan A, et al. Pneumococcal vaccination decreases atherosclerotic lesion formation: molecular mimicry between Streptococcus pneumoniae and oxidized LDL. Nat Med 2003;9:736-43.

30. Liao G, Cheung S, Galeano J, et al. Allopregnanolone treatment delays cholesterol accumulation and reduces autophagic/lysosomal dysfunction and inflammation in Npc1-/- mouse brain. Brain Res 2009;1270:140-51.

31. Griffin EE, Ullery JC, Cox BE, et al. Aggregated LDL and lipid dispersions induce lysosomal cholesteryl ester accumulation in macrophage foam cells. J Lipid Res 2005;46:2052-60.

32. Jerome WG, Yancey PG. The role of microscopy in understanding atherosclerotic lysosomal lipid metabolism. Microsc Microanal 2003;9:54-67.

33. Beltroy EP, Richardson JA, Horton JD, et al. Cholesterol accumulation and liver cell death in mice with Niemann-Pick type C disease. Hepatology 2005;42:886-93.

34. Yu T, Shakkottai VG, Chung C, et al. Temporal and cell-specific deletion establishes that neuronal Npc1 deficiency is sufficient to mediate neurodegeneration. Hum Mol Genet 2011;20:4440-51.

35. Amritraj A, Wang $Y$, Revett TJ, et al. Role of cathepsin D in U18666A-induced neuronal cell death: potential implication in Niemann-Pick type C disease pathogenesis. J Biol Chem 2013;288:3136-52.

36. Walenbergh SM, Houben $T$, Hendrikx $T$, et al. Plasma cathepsin $D$ levels: a novel tool to predict pediatric hepatic inflammation. Am J Gastroenterol 2015;110:462-70.

37. Vanier MT. Niemann-Pick disease type C. Orphanet J Rare Dis 2010;5:16.

38. Okamura N, Kiuchi S, Tamba M, et al. A porcine homolog of the major secretory protein of human epididymis, HE1, specifically binds cholesterol. Biochim Biophys Acta 1999;1438:377-87.

39. Sleat DE, Wiseman JA, El-Banna M, et al. Genetic evidence for nonredundant functional cooperativity between NPC1 and NPC2 in lipid transport. Proc Natl Acad Sci U S A 2004;101:5886-91.

40. Uronen RL, Lundmark P, Orho-Melander M, et al. Niemann-Pick C1 modulates hepatic triglyceride metabolism and its genetic variation contributes to serum triglyceride levels. Arterioscler Thromb Vasc Biol 2010;30:1614-20.

41. Fraley AE, Tsimikas S. Clinical applications of circulating oxidized low-density lipoprotein biomarkers in cardiovascular disease. Curr Opin Lipidol 2006;17:502-9.

42. Walenbergh SM, Koek GH, Bieghs V, et al. Non-alcoholic steatohepatitis: the role of oxidized lowdensity lipoproteins. J Hepatol 2013;58:801-10.

43. Shoji $T$, Nishizawa $Y$, Fukumoto $M$, et al. Inverse relationship between circulating oxidized low density lipoprotein (oxLDL) and anti-oxLDL antibody levels in healthy subjects. Atherosclerosis 2000;148: 171-7.

44. Holvoet P, Kritchevsky SB, Tracy RP, et al. The metabolic syndrome, circulating oxidized LDL, and risk of myocardial infarction in well-functioning elderly people in the health, aging, and body composition cohort. Diabetes 2004;53:1068-73.

45. Porter FD, Scherrer DE, Lanier MH, et al. Cholesterol oxidation products are sensitive and specific blood-based biomarkers for Niemann-Pick C1 disease. Sci TransI Med 2010;2:56ra81.

46. Chalasani N, Deeg MA, Crabb DW. Systemic levels of lipid peroxidation and its metabolic and dietary correlates in patients with nonalcoholic steatohepatitis. Am J Gastroenterol 2004;99:1497-502.

47. Jerome WG, Cox BE, Griffin EE, et al. Lysosomal cholesterol accumulation inhibits subsequent hydrolysis of lipoprotein cholesteryl ester. Microsc Microanal 2008;14:138-49.

48. Maor I, Mandel H, Aviram M. Macrophage uptake of oxidized LDL inhibits lysosomal sphingomyelinase, thus causing the accumulation of unesterified cholesterol-sphingomyelin-rich particles in the lysosomes. A possible role for 7-Ketocholesterol. Arterioscler Thromb Vasc Biol 1995;15:1378-87. 
49. van Leeuwen M, Kemna MJ, de Winther MP, et al. Passive immunization with hypochlorite-oxLDL specific antibodies reduces plaque volume in LDL receptor-deficient mice. PLoS One 2013;8:e68039.

50. Caligiuri G, Khallou-Laschet J, Vandaele M, et al. Phosphorylcholine-targeting immunization reduces atherosclerosis. J Am Coll Cardiol 2007;50:540-6. 


\section{Supplemental figures}

A

Relative body weight gain

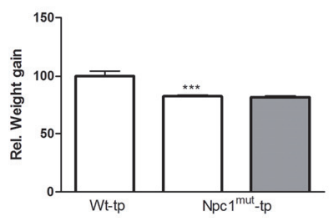

B

Ratio Liver / Total body weight

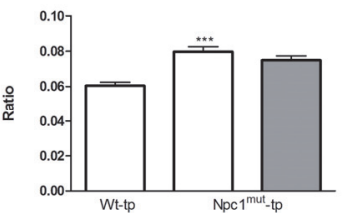

C

Ratio Spleen / Total body weight

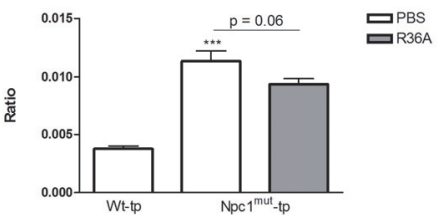

D
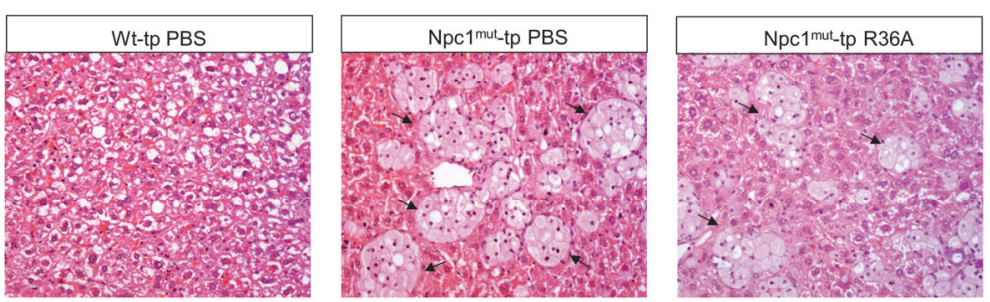

Figure S4.1 Relative body weight gain (A), liver (B), spleen (C) weights and general histology of the liver by a hematoxylin and eosin staining (D) of Wt-tp and (non-)immunized Npc1 ${ }^{\text {mut }}$-tp mice. Arrows indicate macrophage granulomas.
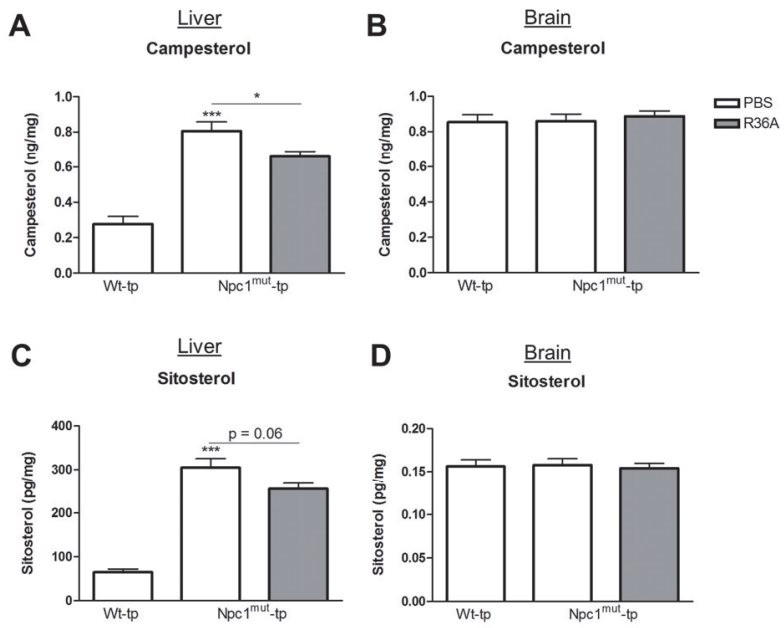

Figure S4.2 Campesterol (A-B) and sitosterol (C-D) levels in brain and liver. 
A

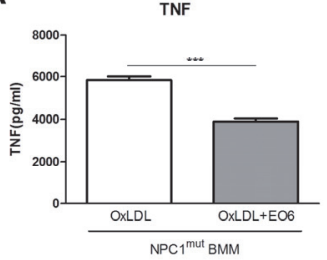

B

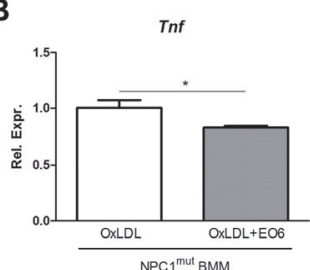

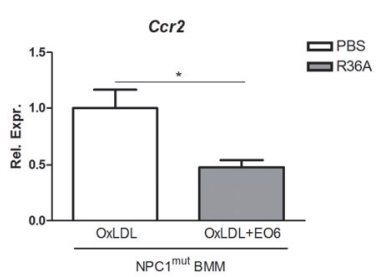

Figure S4.3 TNF protein levels (A) and gene expression of inflammatory-related genes (Tnf and C Cr2) (B) after incubation with oxLDL of NPC1 mutant BMM with or without anti-oxLDL EO6 antibodies. 
Chapter 4 


\section{Chapter 5}

Specific immunization strategies against oxidized low-density lipoprotein: a novel way to reduce non-alcoholic steatohepatitis in mice

V Bieghs, PJ van Gorp, SMA Walenbergh, MJJ Gijbels, F Verheyen, WA Buurman, DE Briles, MH Hofker, CJ Binder, R Shiri-Sverdlov Hepatology 2012;56:894-903 


\section{Abstract}

\section{Background and aims}

Non-alcoholic steatohepatitis (NASH) is characterized by hepatic lipid accumulation combined with inflammation, which can ultimately progress into cirrhosis. Recently, we demonstrated that deletion of scavenger receptors CD36 and SR-A in haematopoietic cells reduced hepatic inflammation. In addition to uptake of modified lipoproteins, CD36 and SR-A are also involved in other functions that can activate the inflammatory response. Therefore, the actual trigger for scavenger receptor activation during NASH is unclear. Here, we hypothesized that hepatic inflammation is triggered by recognition of oxidized LDL (oxLDL) by Kupffer cells (KCs).

\section{Methods}

To inhibit recognition of oxLDL by $K C S, L d l r^{--}$mice were immunized with heat-inactivated pneumococci, which were shown to induce the production of anti-oxLDL IgM antibodies, due to molecular mimicry with oxLDL. The mice received a high-fat, high-cholesterol (HFC) diet during the last 3 weeks to induce NASH.

\section{Results}

Immunization with pneumococci increased anti-oxLDL IgM levels and led to a reduction in hepatic inflammation, as shown by reduced macrophage, neutrophil and T-cell infiltration, and reduced gene expression of Tnf, II-6, II-16, Mcp1 and fibrosis related genes. In immunized mice, $\mathrm{KCs}$ were smaller and showed less cholesterol crystals compared to non-immunized mice.

\section{Conclusions}

Antibodies to oxLDL play an important role in the pathogenesis of NASH. Therefore, the potential of phosphorylcholine-based vaccination strategies as a novel tool for the prevention and therapy of NASH should be tested in the future. 


\section{Introduction}

Non-alcoholic fatty liver disease (NAFLD) is a condition ranging from benign lipid accumulation in the liver (steatosis) to steatosis combined with inflammation. The latter is referred to as non-alcoholic steatohepatitis (NASH). NASH is considered as the hepatic component of metabolic syndrome. Estimates from the United States are that $5.7 \%$ to $17 \%$ of all adults have $\mathrm{NASH}$, while $17 \%$ to $33 \%$ of Americans suffer from NAFLD. ${ }^{1,2}$ As obesity and insulin resistance reach epidemic proportions in industrialized countries, the prevalence of both NAFLD and NASH is increasing. NAFLD is therefore a major health hazard. ${ }^{3}$ Steatosis alone is considered a relatively benign and reversible condition. However, the transition towards NASH represents a key step in the pathogenesis, as it sets the stage for the development of fibrosis, cirrhosis and liver cancer. Although the mechanisms leading to steatosis are well described, little is known about the actual risk factors that drive hepatic inflammation during the progression toward NASH. Consequently, therapeutic options are limited. Therefore, knowledge about the events that lead to hepatic inflammation is of great importance for the diagnosis and treatment of NASH.

Recently, we demonstrated that deletion of scavenger receptors (SRs) CD36 and SR-A in hematopoietic cells reduced hepatic inflammation. ${ }^{4}$ In addition to uptake of modified lipids, SRs are involved in many other inflammatory pathways. These pathways include cellular adhesion, innate immune responses and phagocytosis of apoptotic cells. ${ }^{5}$ Based on the analogy between mechanisms for atherosclerosis and $\mathrm{NASH}$, it is likely that the recognition of oxidized low-density lipoprotein (oxLDL) by Kupffer cells (KCs), rather than other pathways, is the actual trigger for SR-mediated inflammation. Therefore, we hypothesized that hepatic inflammation is triggered by the recognition of oxLDL by KCs.

It has recently been shown that the levels of immunoglobulin $\mathrm{M}$ (IgM) autoantibodies to modified LDL are inversely correlated with atherosclerosis. ${ }^{6-8}$ Oxidation-specific epitopes present in oxLDL are major targets of natural IgM antibodies. ${ }^{9}$ These antibodies arise spontaneously without prior infection or immune exposure and mainly consist of the IgM isotype. ${ }^{10}$ They are produced by innate-like B-1 cells, and provide a first line of defense against bacterial and viral pathogens. ${ }^{11,12}$ In addition, natural IgM antibodies play an important role in providing housekeeping functions by protecting from the accumulation of biological waste, such as oxLDL. ${ }^{10}$ Upon oxidation of $L D L$, reactive oxidation products from phospholipids retain the intact phosphorylcholine (PC) headgroup, which becomes available for immune recognition. These PC headgroups represent one of many so-called oxidation-specific epitopes and are found on the outer side of the membrane of oxLDL. ${ }^{13} \mathrm{~A}$ panel of monoclonal autoantibodies directed to epitopes of oxLDL was cloned from the spleens of apolipoprotein $\mathrm{E}\left(a p o E^{-/}\right)$mice. ${ }^{14}$ In particular, one immunodominant clonotypic set of 
IgM autoantibodies was identified, EO6, which was shown to specifically bind to the PC moiety of oxidized PC-containing phospholipids, such as those present in oxLDL. ${ }^{13}$ EO6 antibodies were found to be identical to the natural T15 antibodies, which are germline-encoded natural antibodies exclusively derived from B-1 cells. These T15 antibodies protect mice against Streptococcus pneumoniae infections, because PC is also present in the capsular polysaccharide of the cell wall of this bacterium. Based on this molecular mimicry, immunization of low-density lipoprotein receptor $\left(\mathrm{Ldlr}^{-/}\right)$mice with heat-killed $S$. pneumonia resulted in higher serum titers of anti-oxLDL IgM antibodies and decreased atherosclerosis. ${ }^{15}$ These findings suggest that anti-oxLDL antibodies directed to the PC group present on oxLDL possibly inhibit the recognition of oxLDL by macrophage SRs, such as CD36.

The aim of the current study was to determine whether oxLDL is causally involved in the pathogenesis of NASH. For this purpose, $\mathrm{Ldll}^{-/-}$mice were used as a wellrecognized model mimicking the human lipoprotein metabolism with high fidelity and is therefore also extremely useful to investigate the physiological triggers for hepatic inflammation, which can already develop upon short term treatment with a high-fat, high-cholesterol (HFC) diet. ${ }^{16}$ These mice were immunized with heat-inactivated pneumococci to investigate whether anti-oxLDL antibodies have a protective effect on $\mathrm{NASH}$. Supporting our hypothesis, immunized $\mathrm{LdIr}^{-/-}$mice showed reduced hepatic inflammation compared to non-immunized mice. These data demonstrate the importance of antibodies to oxLDL in the pathogenesis of NAS. Therefore, the potential of PC-based vaccination strategies as novel tool for the prevention and therapy of NASH should be tested in the future.

\section{Materials and methods}

\section{Preparation immunogen}

For immunization, the heat-inactivated R36A strain of Streptococcus pneumoniae (Birmingham, AL) was used, still bearing the PC headgroup epitope similar to oxLDL. Colonies of the R36A strain were harvested at mid-log phase after incubation at $37^{\circ} \mathrm{C}$ on blood agar plates and transferred to Todd-Hewitt plus $0.5 \%$ yeast broth. The midlog phase is characterized by an optical density (OD) value of 0.425 to 0.45 at $600 \mathrm{~nm}$. S. pneumoniae was heat-inactivated at $60^{\circ} \mathrm{C}$ for 30 minutes; afterwards no colonies of this suspension were detected on blood agar plates. For freezer stocks of strain R36A, small aliquots of S. pneumoniae at mid-log density were harvested and suspended in Todd-Hewitt plus $80 \%$ sterile glycerol and stored at $-80^{\circ} \mathrm{C}^{17}$ 


\section{Mice, immunization, and diet}

$\mathrm{LdIr}^{-/}$mice on a C57BL/6 background were housed under standard conditions and had access to food and water ad libitum. Experiments were performed according to Dutch laws, approved by the Animal Experiment Committee of Maastricht University.

The immunization protocol started in 12-week-old female mice, fed a normal chow diet. Mice were divided into four groups ( $n=10$ for each group) and received the equivalent of $10^{8}$ colony-forming units of the heat-killed pneumococcal immunogen emulsified in $200 \mu \mathrm{l}$ sterile $0.9 \% \mathrm{NaCl}$ for the primary subcutaneous immunization, subsequently three intraperitoneal booster immunizations were administered every 3 weeks. ${ }^{15}$ The control group received a $\mathrm{NaCl}$ injection only. After immunization, the mice were given normal chow, the control group, or an HFC diet, the experimental group, for 3 weeks. Blood from the tail vein was collected after the dietary period and mice were then sacrificed by cervical dislocation. Liver tissue was isolated and snapfrozen in liquid nitrogen and stored at $-80^{\circ} \mathrm{C}$ or fixed in $4 \%$ formaldehyde/PBS. The collection of blood and specimens, the biochemical determination of lipids in plasma and the liver, liver histology, alanine aminotransferase, RNA isolation, complementary DNA synthesis and quantitative PCR and auto-antibody titers against IgG and IgM antibodies to CuOx-LDL and malondialdehyde-LDL were extensively described previously. ${ }^{4}$

Immune complex measurements were performed as described previously. ${ }^{15}$ Briefly, circulating immune complexes were determined by a capture assay in which a polyclonal antibody specific for murine apoB100 was coated on microtiter wells at $5 \mu \mathrm{g} / \mathrm{ml}$ in phosphate-buffered saline. Individual mouse sera (1:100) were added to the wells and incubated for 1 hour at room temperature. IgM bound to the captured apoB-containing particles was detected using an alkaline phosphatase conjugated goat anti-mouse IgM antibody by chemiluminescent enzyme-linked immunosorbent assay. The amount of IgM bound to the captured LDL was then normalized for the amount of captured $\mathrm{apoB}$ and expressed as a ratio of $\operatorname{IgM}$ counts (relative light units/100 ms) to apoB100 counts (relative light units/100 ms) or IgM/apoB.

\section{Electron microscopy}

A detailed overview about the postfixation, embedding, cutting, and type of electron microscope has been described. ${ }^{16}$ To stain the KC lysosomes, acid phosphatase enzyme cytochemistry was performed. Small wedge biopsies of the liver were perfused by syringe injection with ice-cold $2 \%$ purified glutaraldehyde in $0.1 \mathrm{M}$ cacodylate buffer $(\mathrm{pH} \mathrm{7.4)}$ for 15 minutes. The wedge biopsies were cut into small pieces and kept in $0.1 \mathrm{M}$ cacodylate buffer $+7.5 \%$ sucrose at $4^{\circ} \mathrm{C}$ until further processing; the buffer solution was refreshed weekly. The samples were frozen for 1 hour at $-30^{\circ} \mathrm{C}$ whereafter $50-\mu \mathrm{m}$-thick cryosections were made. These sections were incubated according to the cerium-based method of Robinson and Karnovsky for the localization of acid phosphatase. After the incubation, the sections were washed two 
times in $0.1 \mathrm{M}$ cacodylate buffer supplemented with $5 \%$ sucrose, refixed in $3 \%$ glutaraldehyde in cacodylate buffer for 1 hour and rinsed overnight in veronal acetate buffer $\left(\mathrm{pH} 7.4,4^{\circ} \mathrm{C}\right)$. The sections were then postfixed for 30 minutes in $2 \%$ osmium tetroxide in veronal buffer plus $4 \%$ sucrose and then routinely processed for embedding in epon.

\section{Statistical analysis}

Data was statistically analyzed by performing two-tailed nonpaired $t$ tests using GraphPad Prism, version 4.03 for Windows. Data are expressed as the mean \pm SEM and were considered significant at $p<0.05$.

\section{Results}

\section{Increased IgM antibody titers against modified LDL after immunization with heat-inactivated pneumococci}

To determine whether IgM autoantibodies to oxLDL have a protective effect on liver inflammation, mice were immunized for 9 weeks with heat-inactivated pneumococci, known to induce high anti-oxLDL IgM titers dominated by T15-idiotypic IgM. To induce $\mathrm{NASH}$, the mice received an HFC diet during the last 3 weeks. Total body weight and the ratio of liver weight to total body weight were not significantly different between the different groups (Supplemental Figure S5.1). Immunization of $\mathrm{Ldll}^{-/}$mice with heat-inactivated pneumococci resulted in a strong increase in $\operatorname{lgM}$ titers to oxLDL (Figure 5.1A, 5.1B). Only weak but significant IgG responses were observed, consistent with previous reports that pneumococcal immunizations induce an IgM-dominated thymus-independent type-2 response highly specific for PC (Figure 5.1C, 5.1D). The levels of circulating IgM/apoB immune complexes did not differ between the groups, likely indicating efficient clearance of oxLDL (Supplemental Figure S5.2)

\section{No difference in liver lipid levels between immunized and nonimmunized $L d I^{-/-}$mice after 3 weeks of HFC diet}

To investigate liver lipid levels in hyperlipidemic mice with or without immunization, biochemical assessment of liver cholesterol, triglycerides (TGs) and free fatty acids (FFAs) was performed (Figure 5.2A). After 3 weeks on an HFC diet, a clear increase in all liver lipid levels was observed compared to mice on a chow diet. Liver lipid levels did not differ between immunized and nonimmunized $L d / r^{-/}$mice on the HFC diet. Mice on the chow diet showed a small increase in liver lipid levels after immunization when compared with nonimmunized $L d l r^{-/}$mice. Oil red $\mathrm{O}$ and hematoxylin and eosin staining confirmed the biochemical liver lipid measurements (Figure 5.2B-E and Supplemental Figure S5.3). 


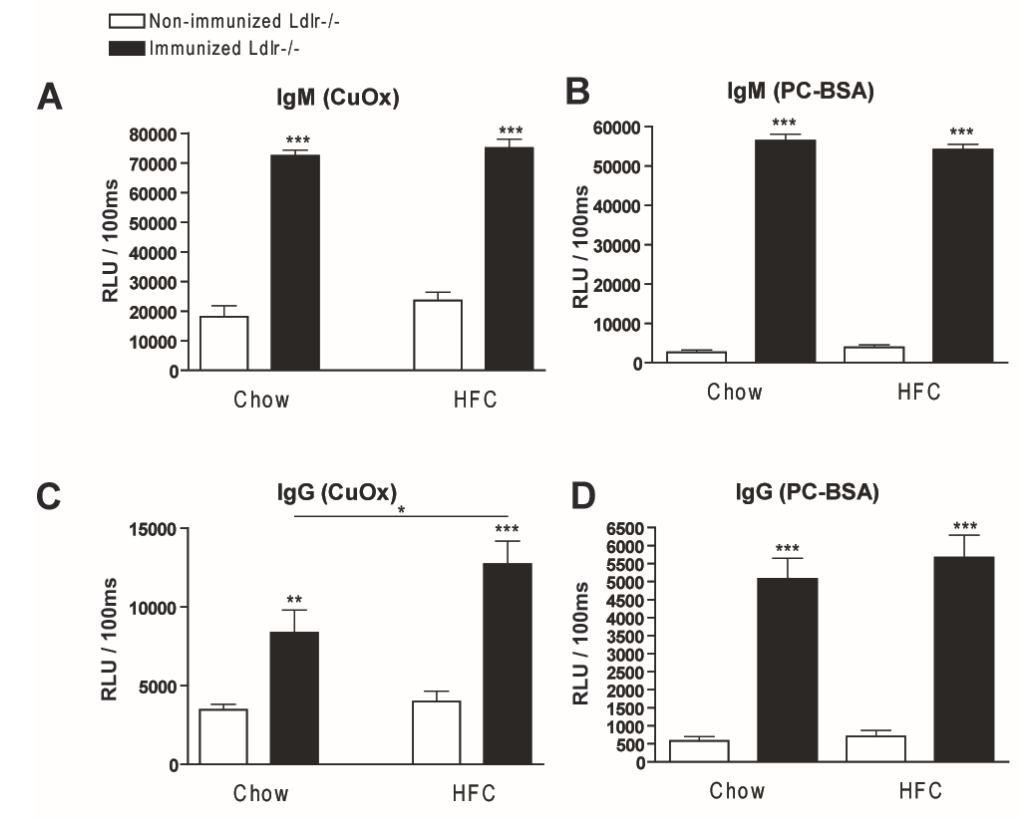

Figure 5.1 IgM autoantibodies in mice that received pneumococcal immunization. (A-D) IgM and IgG antibodies against oxLDL (CUOx and PC-BSA) were measured in plasma of pneumococciimmunized $(n=10)$ and control $(n=10)$ mice at a dilution of $1: 200$, respectively. Data are expressed as relative light units (RLU) / $100 \mathrm{~ms}$ and were triplicate determinations. ${ }^{*} p<0.05$; $* * p<0.01 ; * * p<0.001$.

\section{Decreased plasma cholesterol in immunized $L d I r^{-/-}$mice on an HFC diet compared with control mice}

The effect of immunization on plasma lipids was assessed by measuring the levels of plasma cholesterol, TGs and FFAs. After feeding on the HFC diet, a significant increase was observed for all plasma lipids compared with mice on a chow diet. Interestingly, plasma cholesterol was reduced in immunized $L d l r^{-/-}$mice compared to nonimmunized mice on an HFC diet. Plasma TGs and FFAs did not differ between the groups following an HFC diet. On chow diet, plasma lipid levels did not differ between the groups (Figure 5.3). 


\section{A}

$\square$ Non-immunized Ldlr-/.

Immunized Ldir-/.

Liver Cholesterol
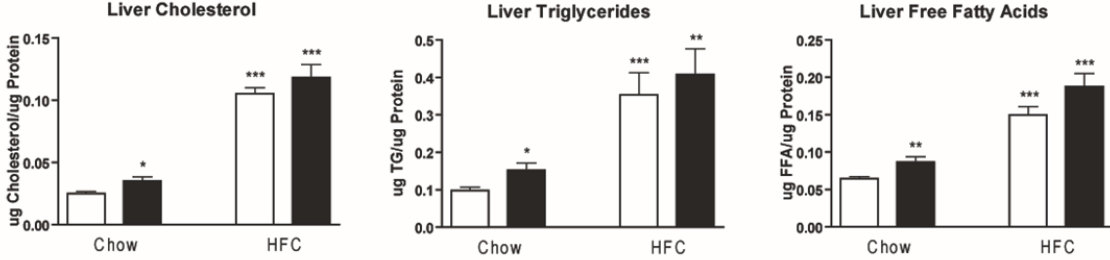

B

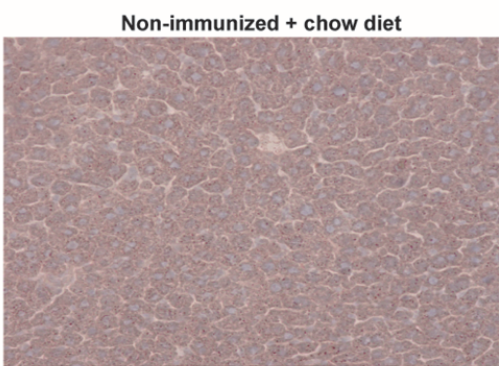

C Immunized + chow diet
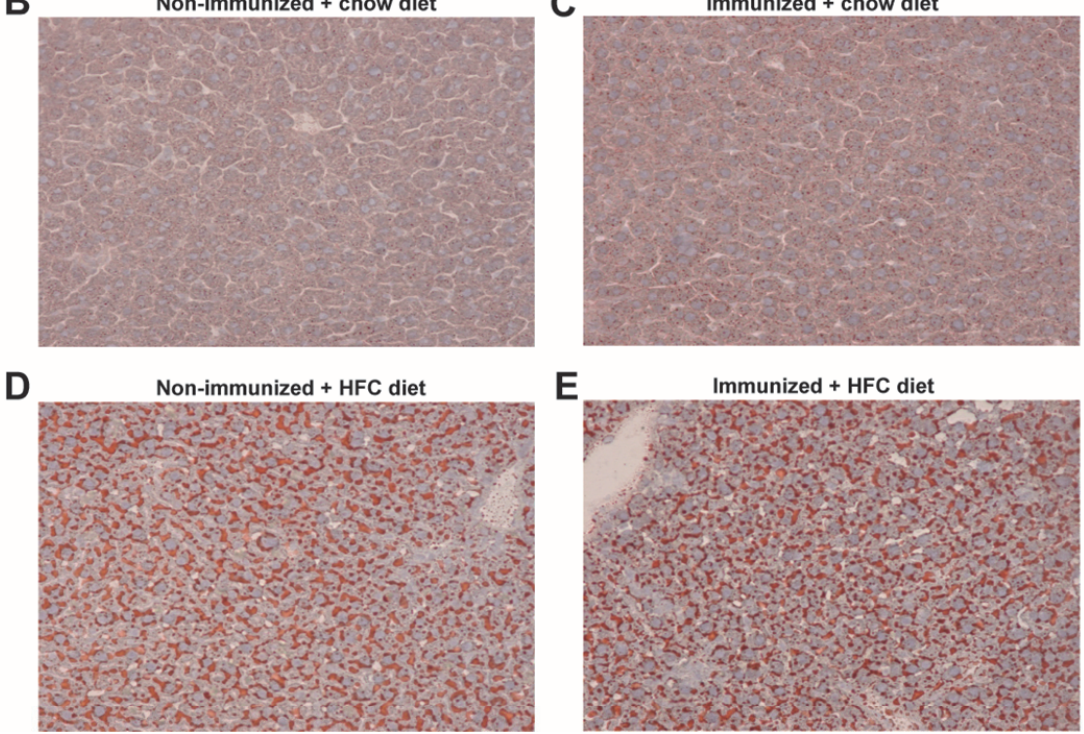

Figure 5.2 Liver lipid levels. (A) Liver cholesterol, TGs and FFAs after chow and 3 weeks of an HFC diet. (B-E) Oil red $O$ staining after 3 weeks of an HFC diet in (B,D) nonimmunized (C,E) and immunized $\mathrm{Ldll}^{-/-}$mice after $(\mathbf{B}, \mathbf{C})$ chow and $(\mathbf{D}, \mathbf{E}) 3$ weeks of feeding on an HFC diet, respectively. Asteriks indicate significant difference from nonimmunized mice on a chow diet. $* p<0.05 ; * * p<0.01 ; * * *<<0.001$.

\section{$\square$ Non-immunized Ldir--} Immunized Ldir-/-
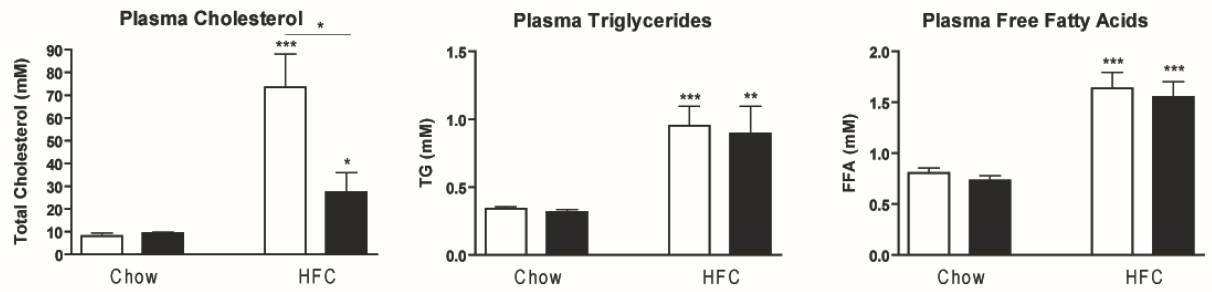

Figure 5.3 Plasma lipid levels. Plasma cholesterol, TGs and FFAs after a chow diet and 3 weeks of an HFC diet in nonimmunized and immunized $L d l r^{-/}$mice. Asteriks indicate significant difference from nonimmunized mice on a chow diet. ${ }^{*} p<0.05 ;{ }^{* *} p<0.01 ; * * p<0.001$. 


\section{Decreased hepatic inflammation in $\mathrm{LdIr}^{-/-}$mice immunized with heat- inactivated pneumococci}

To determine whether immunization of $\mathrm{Ld}_{\mathrm{I}} \mathrm{r}^{-/}$mice with heat-inactivated pneumococci affects hepatic inflammation, liver sections were stained for the inflammatory cell markers Mac-1 (infiltrated macrophages and neutrophils), NIMP (neutrophils) and CD3 ( $T$ cells). As shown in Figure 5.4A, the number of infiltrated macrophages, neutrophils, and T cells was lower in immunized $\mathrm{Ldll}^{-1-}$ mice compared with nonimmunized mice after feeding on an HFC diet. These data on cell infiltration are confirmed by hematoxylin and eosin staining (Supplemental Figure S5.3). Moreover, the normal chow diet induced a significant increase in the number of neutrophils in immunized chow-fed mice compared with nonimmunized mice. Representative histological pictures of the Mac-1 staining for all four experimental groups are shown in Figure 5.4B-E. Further confirming the reduced hepatic inflammation in immunized $\mathrm{Ldll}^{-/-}$mice on the HFC diet, gene expression analysis showed a significant decrease in the inflammatory markers tumor necrosis factor (Tnf), interleukin-1beta (II-1b), interleukin 6 (II-6) and monocyte chemoattractant protein 1 (Mcp1) in livers of immunized $L d l r^{-/-}$mice on an HFC diet compared with nonimmunized mice (Figure 5.4F). However, hepatic inflammation in $\mathrm{Ldll}^{-/-}$mice on an HFC diet after immunization was still higher than chow-fed immunized mice according to the inflammatory markers Tnf, $\|-1 b$ and Mcp1. The presence of elevated transaminases in plasma-like alanine aminotransferase (ALT) did not differ between the different groups (Supplemental Figure S5.4).

\section{After 3 weeks of HFC diet, immunization prevented expression of fibrosis-related genes in $\mathrm{Ldll}^{-/-}$mice}

Fibrosis is considered an advanced stage of NASH. Collagen staining (Sirius Red) was performed to determine the degree of fibrosis. No differences were observed between the experimental groups after 3 weeks of an HFC diet (Figure 5.5A), which is probably related to the short duration of the HFC diet. However, gene expression analysis for collagen type $1 \mathrm{~A} 1$ (Col1A1) and transforming growth factor beta (Tgf- 6 ) demonstrated that the messenger RNA levels of these fibrogenic genes were lower in immunized mice compared with nonimmunized mice on an HFC diet (Figure 5.5B). 
A
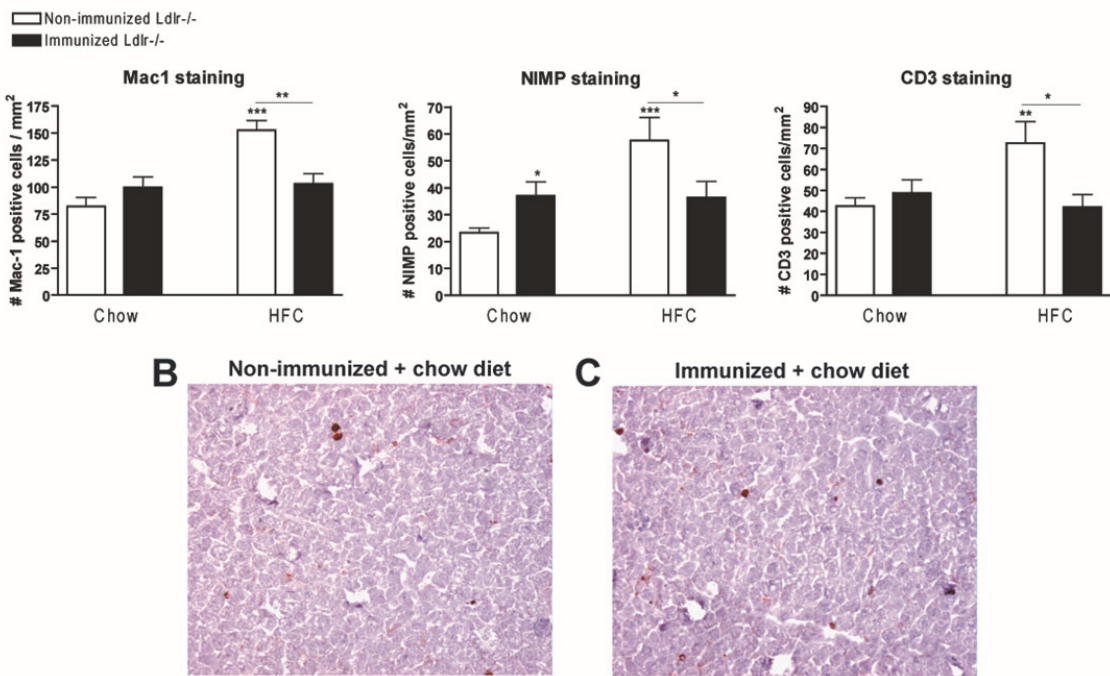

C Immunized + chow diet
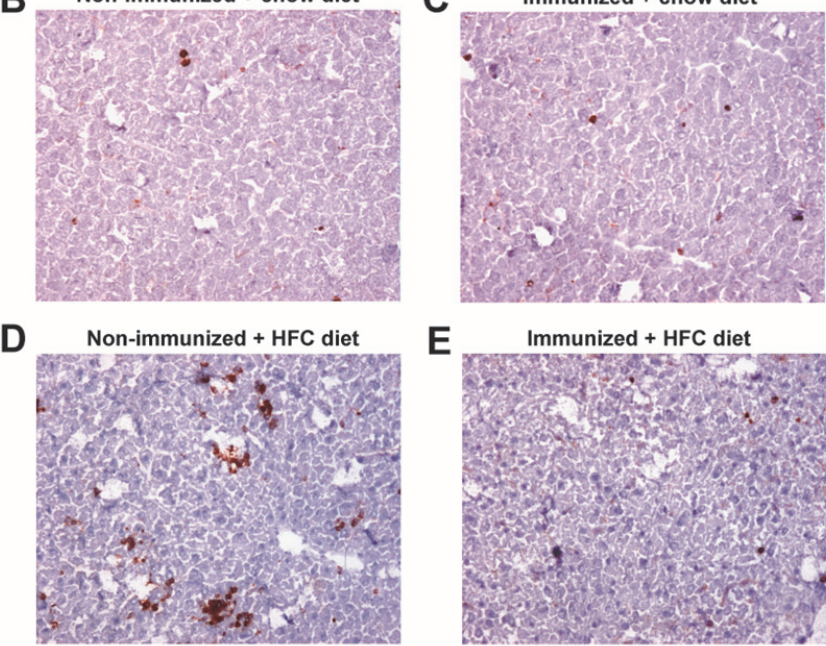

$\mathbf{F}$
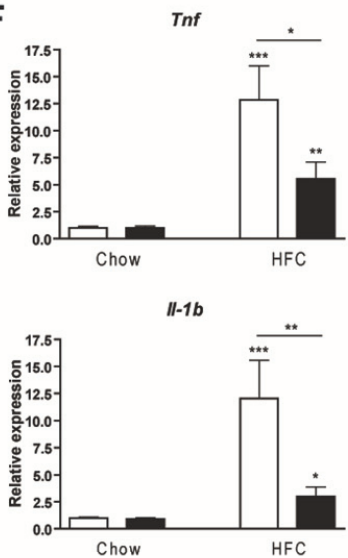

E

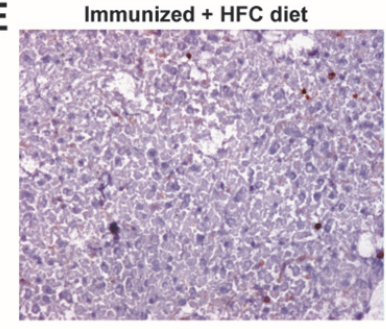

II-6
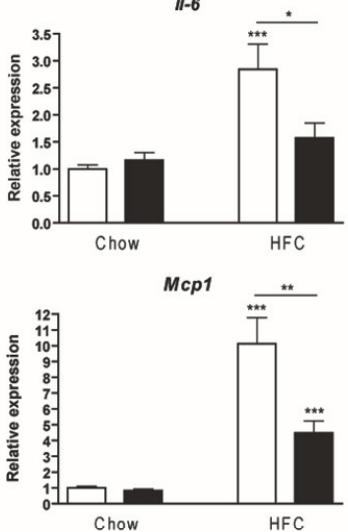

Figure 5.4 Parameters of hepatic inflammation. (A) Liver sections were stained for infiltrated macrophages and neutrophils (Mac-1), neutrophils (NIMP), and T cells (CD3), and counted. (B-E) Representative images of Mac-1 staining (magnification $\times 200$ ) after feeding on a chow $(\mathbf{B}, \mathbf{C})$ and an HFC diet (D,E) in nonimmunized (B,D) and immunized (C,E) $\mathrm{Ldlr}^{-/}$mice, respectively. (F) Gene expression analysis for Tnf, $/ 16, \| 16$ and Mcp1. Asteriks indicate significant difference from nonimmunized mice on a chow diet. ${ }^{*} p<0.05 ;{ }^{* *} p<0.01$; $* * * \mathrm{p}<0.001$ 
A

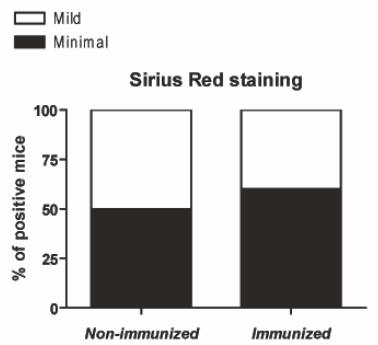

B

$\square$ Non-immunized Ldir-l

Immunized Ldir-I-

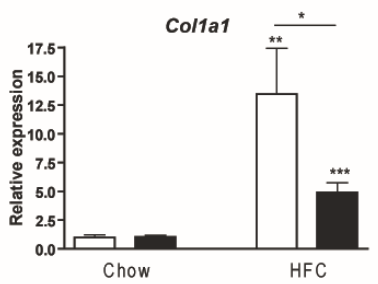

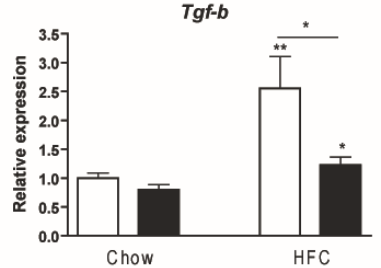

Figure 5.5 Parameters of hepatic fibrosis. (A) Quantification of sirius red (collagen) after 3 weeks of an HFC diet. Livers were quantified as minimally, mildly or moderately positive for collagen around and in between the blood vessels of the liver. (B) Gene expression analysis of the fibrosis markers, collagen (Col1a1) and Tgf- 6 . Asteriks indicate significant difference from nonimmunized mice on a chow diet. ${ }^{*} \mathrm{p}<0.05 ;{ }^{*} \mathrm{p}<0.01 ;{ }^{* * *} \mathrm{p}<0.001$.

\section{Decreased foamy appearance of KCs in immunized $\mathrm{LdII}^{-/-}$mice on an HFC diet}

Immunohistochemistry for CD68 was performed to characterize the KCs. Scoring of the $\mathrm{CD} 68$ positive sections revealed a reduction in size of foamy KCs in immunized $\mathrm{LdIr}^{-/}$mice compared with nonimmunized mice on an HFC diet (Figure 5.6A-C). Gene expression of $C d 68$ was reduced in the immunized $L d l r^{-/}$mice compared with nonimmunized mice on an HFC diet (Figure 5.6B). Electron microscopy of KCs confirmed the differences in size of the KCs between immunized and nonimmunized $\mathrm{Ld}_{\mathrm{I}} \mathrm{r}^{-/}$mice, and showed that the immunized mice on an HFC diet had less lysosomal cholesterol accumulation and cholesterol crystals compared with nonimmunized mice (Figure 5.6D). 
A

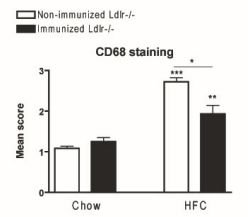

C Non-immunized + chow diet

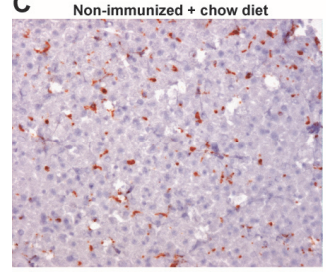

Non-immunized + HFC diet

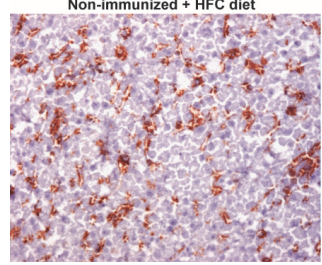

B

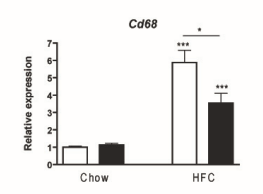

Immunized + chow diet

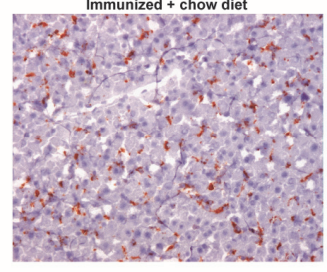

Immunized + HFC diet

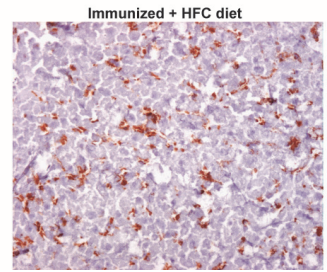

D
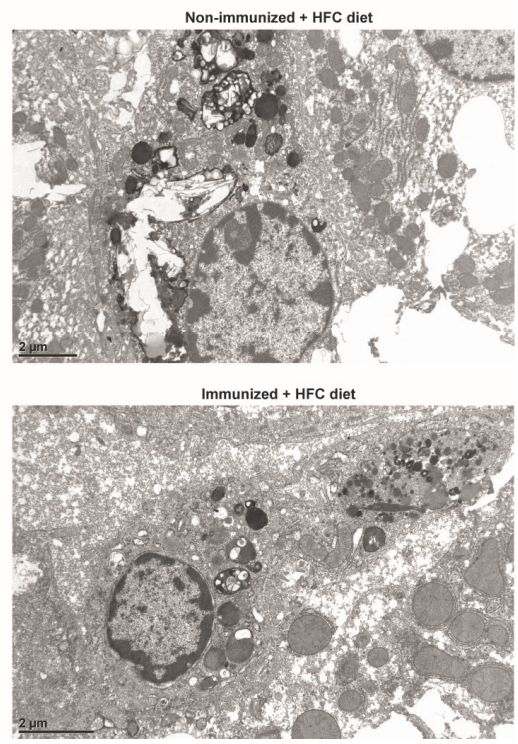

Figure 5.6 Foamy KCs. (A) Liver sections were stained for CD68 (KCs) and scored for the level of foamy appearance: 1 (mild foamy appearance) to 3 (severe foamy appearance). Mean scores were calculated from six microscopic views. (B) Gene expression analysis of the KC activation

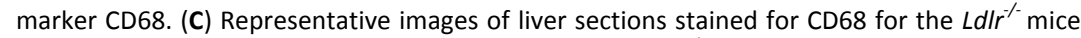
on a chow diet without and with immunization and for $L d l r^{-/}$mice on an HFC diet without and with immunization, respectively (magnification x200). (D) Electron microscopy of foamy KCs. Acid phosphatase staining indicating the lysosomes of the KCs in nonimmunized and immunized $\mathrm{Ldll}^{-/}$mice on an HFC diet. Asteriks indicate significant difference from nonimmunized mice on a chow diet. ${ }^{*} \mathrm{p}<0.05 ;{ }^{*} \mathrm{p}<0.01 ;{ }^{* *} \mathrm{p}<0.001$.

\section{Discussion}

Until now, the actual risk factors that drive hepatic inflammation during the progression to NASH were unknown. To determine whether oxLDL is causally involved in the pathogenesis of NASH, serum anti-oxLDL IgM antibody levels were increased by immunizing $\mathrm{Ldll}^{-/}$mice with heat-inactivated pneumococci, which dramatically decreased hepatic inflammation. These data point toward oxLDL as a trigger for hepatic inflammation. Furthermore, our data suggest that PC-based vaccination strategies could be the basis for a vaccination protocol for NASH therapy. However, the long-term consequences of immunization are unknown at the moment and should be tested in the future.

SR-mediated uptake of oxLDL by macrophages sets off a cascade of pro-inflammatory events leading to the initiation of the inflammatory response. OxLDL is phagocytosed 
by macrophages via binding of the oxPC molecules present in oxLDL to macrophage SRs, and results in foam cells. ${ }^{18,19}$ Furthermore, CD36 has been implicated in inflammatory signaling induced by oxLDL. ${ }^{20}$ Previously, we have shown that both CD36 and SR-A play an important role in diet-induced NASH. ${ }^{4}$ Because SRs have a wide spectrum of functions, ${ }^{21-23}$ it is not clear whether the recognition of modified lipoproteins is the actual trigger for hepatic inflammation during NASH. Our data demonstrate for the first time that inflammation is reduced in the livers of pneumococci-immunized mice. These results are in line with earlier findings demonstrating decreased atherosclerotic lesion formation after pneumococcal immunization. ${ }^{15}$ Similarly, apoE ${ }^{\%}$ mice immunized with PC, one of the epitopes of anti-oxLDL autoantibodies present in oxLDL but also in the CPS of S. pneumoniae, demonstrated an increase in anti-oxLDL autoantibodies together with a reduction in atherosclerotic lesions. ${ }^{24}$ Several in vitro studies suggest that the induced IgM antibodies against oxLDL prevented binding and uptake of oxLDL by macrophages and/or neutralize its proinflammatory signaling. ${ }^{11,15,25,26}$ Indeed, the inflammatory process associated with atherosclerotic plaque formation is linked to the cytotoxicity and macrophage chemo-attractivity of oxLDL. Moreover, oxLDL is thought to be an atherogenic factor, because its uptake by macrophages results in the formation of foam cells, the hallmark cells of atherosclerotic lesions. ${ }^{18,19,27}$ Our data provide evidence for similar mechanisms between atherosclerosis and NASH. Thus, the reduced inflammation in mice in which the SRs on hematopoietic cells had been deleted is likely to be related to the reduced recognition of oxLDL by KCs.

Interestingly, plasma cholesterol levels were significantly reduced in our immunized $\mathrm{Ld}_{\mathrm{I}} \mathrm{r}^{-/}$mice. Previously, it was shown that anti-oxLDL antibodies directed to the PC group present on oxLDL inhibit the recognition of oxLDL by macrophage SRs. ${ }^{28}$ We speculate that the formed immune complexes containing both LDL and oxLDL particles may be cleared faster by alternative pathways, as this is the case for IgM mediating apoptotic cell clearance. However, measurements of $\operatorname{lgM} / \mathrm{apoB}$ immune complexes indicated that there were no differences between our groups of $\mathrm{LdIr}^{-/}$ mice, also suggesting that the induced IgM mediate their protective effect by directly neutralizing the proinflammatory effects of oxLDL. These findings are in line with Binder et al., ${ }^{15}$ who demonstrated that under these conditions, no differences in immune complexes were observed. It is possible that the protective effect of these antibodies in vivo is further enhanced via a reduction in plasma cholesterol levels, since plasma cholesterol levels are an important trigger for hepatic inflammation. ${ }^{16}$

NASH patients are often associated with high levels of lipid peroxidation products such as those present in oxLDL. Therefore, it has been suggested that the elevated levels of lipid peroxidation might make an important contribution to the pathogenesis of NASH. ${ }^{29,30}$ In the literature, it is demonstrated that the presence of immune responses toward lipid peroxidation products can be a predictor of progression of 
NAFLD. ${ }^{31}$ In addition, it was demonstrated that oxidized phosphatidylcholines were found predominantly in steatotic hepatocytes and macrophages/KCs and were more abundant in NAFLD/NASH livers than in normal control livers. ${ }^{32}$ Moreover, we have shown that NASH patients display increased hepatic myeloperoxidase activity, which is also associated with lipid peroxidation. ${ }^{33}$ The role of oxidative stress as a key factor contributing to hepatic injury in patients with $\mathrm{NASH}^{34,35}$ has been underlined by a study with vitamin $\mathrm{E}$ therapy of nondiabetic NASH patients. ${ }^{36}$

Because fibrosis is one of the later consequences of NASH, we investigated the effect of immunization with heat-inactivated pneumococci on hepatic fibrosis. Gene expression of fibrosis-related genes was decreased, yet not confirmed by sirius red staining. This is probably due to the short period of 3 weeks on an HFC diet, because $\mathrm{LdIr}^{-/}$mice only develop fibrosis after 3 months on a mild atherogenic diet. ${ }^{4}$ However, we report for the first time that uptake of oxLDL is associated with fibrogenesis in vivo. In line with these observations, a study by Kang et al. demonstrated that oxLDL can activate hepatic stellate cells in vitro. ${ }^{37}$ These findings indicate a crucial role for oxLDL in the fibrogenic process.

As expected, immunized mice on an HFC diet showed decreased foamy KCs compared with nonimmunized mice. This reduction in size is probably due to decreased plasma cholesterol levels, as the size of the foamy KCs is not always correlated with the inflammatory state of the liver. ${ }^{4,16}$ Overloading of macrophages with oxLDL was shown to lead to the formation of cholesterol monohydrate crystals. ${ }^{38}$ In line with these findings, we showed that after immunization with heat-inactivated pneumococci, KCs were less foamy, had less lysosomal cholesterol accumulation and therefore also less cholesterol crystals. These data indicate that the increased cholesterol accumulation inside KCs, together with the crystallization, is linked to hepatic inflammation.

To date, no therapy for NASH is available. Our novel data in mice suggest that future research should focus on oxLDL as a trigger for NASH. Therefore, the potential of PCbased vaccination strategies to be used as a novel tool for the prevention and therapy of NASH should be tested in the future. 


\section{References}

1. McCullough AJ. The clinical features, diagnosis and natural history of nonalcoholic fatty liver disease. Clin Liver Dis 2004;8:521-33, viii.

2. Clark JM, Diehl AM. Defining nonalcoholic fatty liver disease: implications for epidemiologic studies. Gastroenterology 2003;124:248-50.

3. Parekh S, Anania FA. Abnormal lipid and glucose metabolism in obesity: implications for nonalcoholic fatty liver disease. Gastroenterology 2007;132:2191-207.

4. Bieghs V, Wouters K, van Gorp PJ, Gijbels MJ, de Winther MP, Binder CJ, Lutjohann D, et al. Role of scavenger receptor $\mathrm{A}$ and $\mathrm{CD} 36$ in diet-induced nonalcoholic steatohepatitis in hyperlipidemic mice. Gastroenterology;138:2477-2486, 2486 e2471-3.

5. Yamada Y, Doi T, Hamakubo T, Kodama T. Scavenger receptor family proteins: roles for atherosclerosis, host defence and disorders of the central nervous system. Cell Mol Life Sci 1998;54: 628-40.

6. Karvonen J, Paivansalo M, Kesaniemi YA, Horkko S. Immunoglobulin M type of autoantibodies to oxidized low-density lipoprotein has an inverse relation to carotid artery atherosclerosis. Circulation 2003;108:2107-12.

7. Tsimikas S, Brilakis ES, Lennon RJ, Miller ER, Witztum JL, McConnell JP, Kornman KS, et al. Relationship of IgG and IgM autoantibodies to oxidized low density lipoprotein with coronary artery disease and cardiovascular events. J Lipid Res 2007;48:425-33.

8. Horkko S, Bird DA, Miller E, Itabe H, Leitinger N, Subbanagounder G, Berliner JA, et al. Monoclonal autoantibodies specific for oxidized phospholipids or oxidized phospholipid-protein adducts inhibit macrophage uptake of oxidized low-density lipoproteins. J Clin Invest 1999;103:117-28.

9. Chou MY, Fogelstrand L, Hartvigsen K, Hansen LF, Woelkers D, Shaw PX, Choi J, et al. Oxidationspecific epitopes are dominant targets of innate natural antibodies in mice and humans. J Clin Invest 2009;119:1335-49.

10. Lutz HU, Binder CJ, Kaveri S. Naturally occurring auto-antibodies in homeostasis and disease. Trends Immunol 2009;30:43-51.

11. Binder CJ, Silverman GJ. Natural antibodies and the autoimmunity of atherosclerosis. Springer Semin Immunopathol 2005;26:385-404.

12. Baumgarth $\mathrm{N}$. The double life of a B-1 cell: self-reactivity selects for protective effector functions. Nat Rev Immunol;11:34-46.

13. Shaw PX, Horkko S, Chang MK, Curtiss LK, Palinski W, Silverman GJ, Witztum JL. Natural antibodies with the T15 idiotype may act in atherosclerosis, apoptotic clearance, and protective immunity. J Clin Invest 2000;105:1731-40.

14. Palinski W, Horkko S, Miller E, Steinbrecher UP, Powell HC, Curtiss LK, Witztum JL. Cloning of monoclonal autoantibodies to epitopes of oxidized lipoproteins from apolipoprotein E-deficient mice. Demonstration of epitopes of oxidized low density lipoprotein in human plasma. J Clin Invest 1996;98: 800-14.

15. Binder CJ, Horkko S, Dewan A, Chang MK, Kieu EP, Goodyear CS, Shaw PX, et al. Pneumococcal vaccination decreases atherosclerotic lesion formation: molecular mimicry between Streptococcus pneumoniae and oxidized LDL. Nat Med 2003;9:736-43.

16. Wouters K, van Gorp PJ, Bieghs V, Gijbels MJ, Duimel H, Lutjohann D, Kerksiek A, et al. Dietary cholesterol, rather than liver steatosis, leads to hepatic inflammation in hyperlipidemic mouse models of nonalcoholic steatohepatitis. Hepatology 2008;48:474-86.

17. Briles DE, Forman C, Hudak S, Claflin JL. Anti-phosphorylcholine antibodies of the T15 idiotype are optimally protective against Streptococcus pneumoniae. J Exp Med 1982;156:1177-85.

18. Itabe H, Suzuki K, Tsukamoto Y, Komatsu R, Ueda M, Mori M, Higashi Y, et al. Lysosomal accumulation of oxidized phosphatidylcholine-apolipoprotein B complex in macrophages: intracellular fate of oxidized low density lipoprotein. Biochim Biophys Acta 2000;1487:233-45.

19. Ehara S, Ueda M, Naruko T, Haze K, Itoh A, Otsuka M, Komatsu R, et al. Elevated levels of oxidized low density lipoprotein show a positive relationship with the severity of acute coronary syndromes. Circulation 2001;103:1955-60. 
20. Stewart CR, Stuart LM, Wilkinson K, van Gils JM, Deng J, Halle A, Rayner KJ, et al. CD36 ligands promote sterile inflammation through assembly of a Toll-like receptor 4 and 6 heterodimer. Nat Immunol;11:155-61.

21. Platt N, Suzuki H, Kurihara Y, Kodama T, Gordon S. Role for the class A macrophage scavenger receptor in the phagocytosis of apoptotic thymocytes in vitro. Proc Natl Acad Sci U S A 1996;93: 12456-60.

22. Febbraio M, Hajjar DP, Silverstein RL. CD36: a class B scavenger receptor involved in angiogenesis, atherosclerosis, inflammation, and lipid metabolism. J Clin Invest 2001;108:785-91.

23. Cotena A, Gordon S, Platt N. The class A macrophage scavenger receptor attenuates CXC chemokine production and the early infiltration of neutrophils in sterile peritonitis. J Immunol 2004;173: 6427-32.

24. Caligiuri G, Khallou-Laschet J, Vandaele M, Gaston AT, Delignat S, Mandet C, Kohler HV, et al. Phosphorylcholine-targeting immunization reduces atherosclerosis. J Am Coll Cardiol 2007;50:540-6.

25. Binder CJ, Chang MK, Shaw PX, Miller YI, Hartvigsen K, Dewan A, Witztum JL. Innate and acquired immunity in atherogenesis. Nat Med 2002;8:1218-26.

26. Chang MK, Bergmark C, Laurila A, Horkko S, Han KH, Friedman P, Dennis EA, et al. Monoclonal antibodies against oxidized low-density lipoprotein bind to apoptotic cells and inhibit their phagocytosis by elicited macrophages: evidence that oxidation-specific epitopes mediate macrophage recognition. Proc Natl Acad Sci U S A 1999;96:6353-8.

27. Kayo S, Ohsawa M, Ehara S, Naruko T, Ikura Y, Hai E, Yoshimi N, et al. Oxidized low-density lipoprotein levels circulating in plasma and deposited in the tissues: comparison between Helicobacter pyloriassociated gastritis and acute myocardial infarction. Am Heart J 2004;148:818-25.

28. Hartvigsen K, Chou MY, Hansen LF, Shaw PX, Tsimikas S, Binder CJ, Witztum JL. The role of innate immunity in atherogenesis. J Lipid Res 2009;50 Suppl:S388-93.

29. Chalasani N, Deeg MA, Crabb DW. Systemic levels of lipid peroxidation and its metabolic and dietary correlates in patients with nonalcoholic steatohepatitis. Am J Gastroenterol 2004;99:1497-502.

30. James O, Day C. Non-alcoholic steatohepatitis: another disease of affluence. Lancet 1999;353:1634-6.

31. Albano E, Mottaran E, Vidali M, Reale E, Saksena S, Occhino G, Burt AD, et al. Immune response towards lipid peroxidation products as a predictor of progression of non-alcoholic fatty liver disease to advanced fibrosis. Gut 2005;54:987-93.

32. Ikura $Y$, Ohsawa $M$, Suekane $T$, Fukushima $H$, Itabe $H$, Jomura $H$, Nishiguchi $S$, et al. Localization of oxidized phosphatidylcholine in nonalcoholic fatty liver disease: impact on disease progression. Hepatology 2006;43:506-14.

33. Rensen SS, Slaats Y, Nijhuis J, Jans A, Bieghs V, Driessen A, Malle E, et al. Increased hepatic myeloperoxidase activity in obese subjects with nonalcoholic steatohepatitis. Am J Pathol 2009;175: 1473-82.

34. Sanyal AJ, Campbell-Sargent C, Mirshahi F, Rizzo WB, Contos MJ, Sterling RK, Luketic VA, et al. Nonalcoholic steatohepatitis: association of insulin resistance and mitochondrial abnormalities. Gastroenterology 2001;120:1183-92.

35. McClain CJ, Mokshagundam SP, Barve SS, Song Z, Hill DB, Chen T, Deaciuc I. Mechanisms of nonalcoholic steatohepatitis. Alcohol 2004;34:67-79.

36. Sanyal AJ, Chalasani N, Kowdley KV, McCullough A, Diehl AM, Bass NM, Neuschwander-Tetri BA, et al. Pioglitazone, vitamin E, or placebo for nonalcoholic steatohepatitis. N Engl J Med;362:1675-85.

37. Kang $Q$, Chen A. Curcumin eliminates oxidized LDL roles in activating hepatic stellate cells by suppressing gene expression of lectin-like oxidized LDL receptor-1. Lab Invest 2009;89:1275-90.

38. Tangirala RK, Jerome WG, Jones NL, Small DM, Johnson WJ, Glick JM, Mahlberg FH, et al. Formation of cholesterol monohydrate crystals in macrophage-derived foam cells. J Lipid Res 1994;35:93-104. 


\section{Supplemental figures}

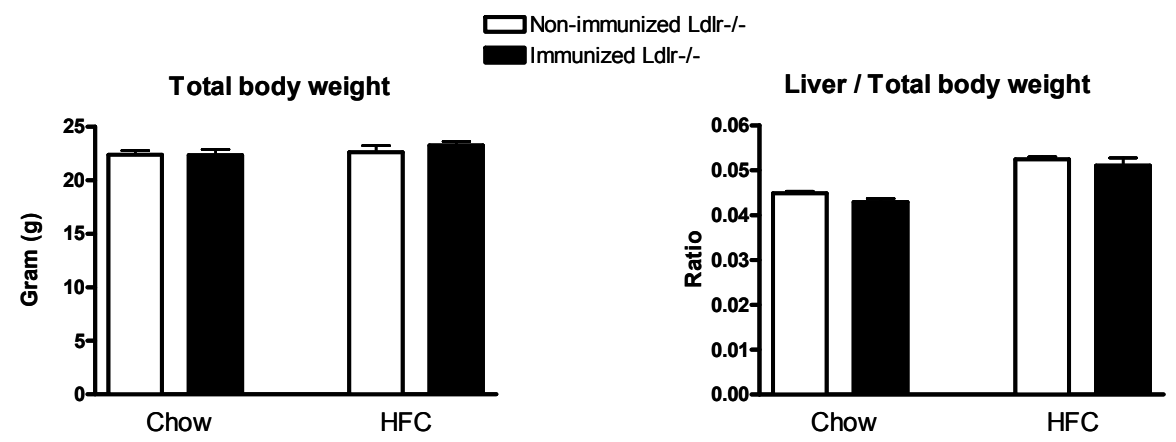

Figure S5.1 Weight. (A) Total body weight and (B) ratio of liver weight to total body weight in in nonimmunized and immunized $\mathrm{Ldll}^{-/}$mice on an HFC diet.

Immune complexes IgM / apoB

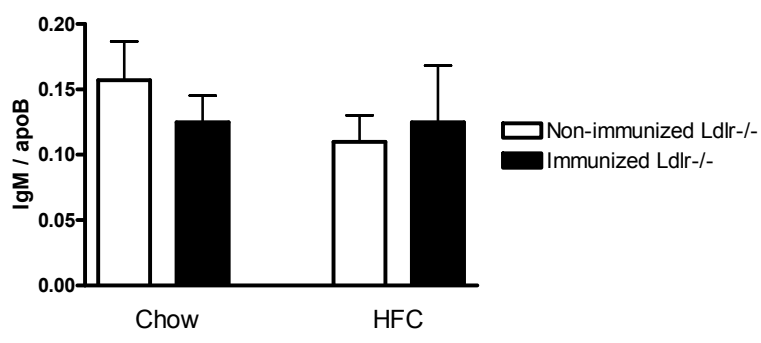

Figure S5.2 Circulating immune complexes in plasma. The ratio of IgM/apoB immune complexes in nonimmunized and immunized $L d l r^{--}$mice on chow and HFC diet. 


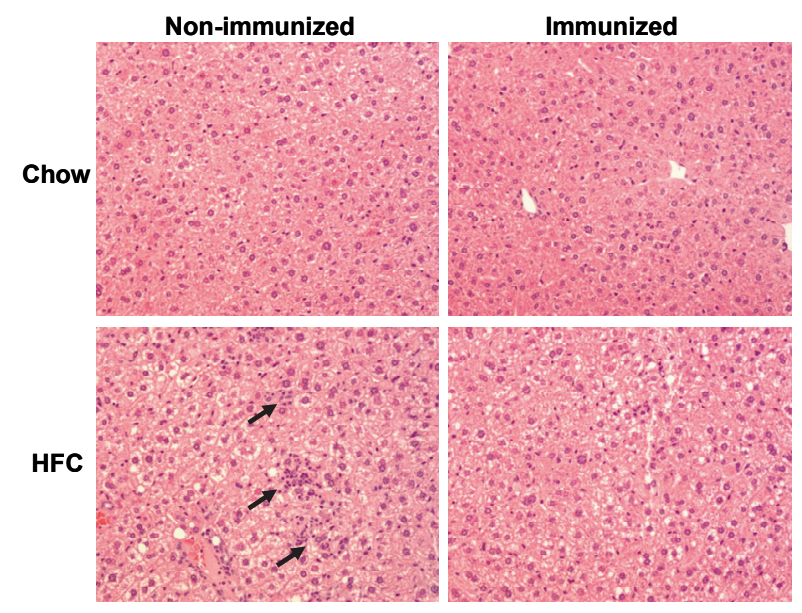

Figure S5.3 General liver histology. Representative pictures (magnification x200) of hematoxylin an eosin staining after chow and 3 weeks of HFC diet in nonimmunized and immunized $\mathrm{Ldlr}^{-/}$mice.

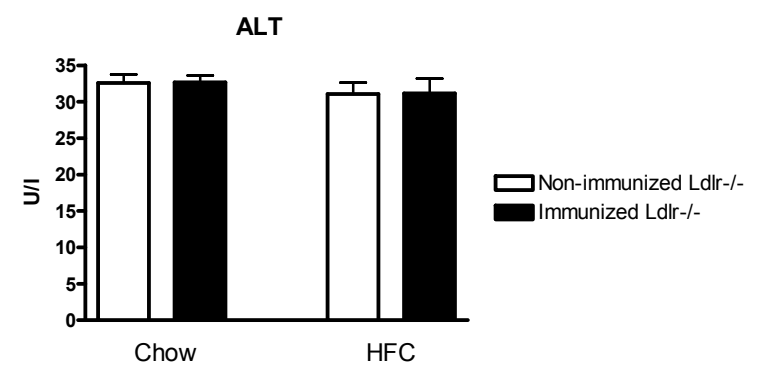

Figure S5.4 Plasma alanine aminotransferase levels. Aminotransferase (ALT) levels in plasma of nonimmunized and immunized $L d l r^{--}$mice after chow and HFC feeding. 


\section{Chapter \\ 6}

\section{Plasma cathepsin D levels: a novel tool to predict pediatric hepatic inflammation}

SMA Walenbergh, T Houben, T Hendrikx, MLJ Jeurissen, PJ van Gorp, ACE Vreugdenhil, MP Adriaanse, WA Buurman, MH Hofker, A Mosca, PJ Lindsey, A Alisi, D Liccardo, N Panera, GH Koek, V Nobili, R Shiri-Sverdlov Am J Gastroenterol 2015;110:462-70 


\section{Abstract}

\section{Background and aims}

Non-alcoholic steatohepatitis (NASH) is the most severe form of a hepatic condition known as non-alcoholic fatty liver disease (NAFLD). NASH is histologically characterized by hepatic fat accumulation, inflammation and ballooning, and eventually coupled with fibrosis, which in turn may progress to end-stage liver disease even in young individuals. Hence, there is a critical need for specific non-invasive markers to predict hepatic inflammation at an early age. We investigated if plasma levels of cathepsin D (CatD), a lysosomal protease, correlated with severity of liver inflammation in pediatric NAFLD.

\section{Methods}

Liver biopsies from children ( $n=96$ ) with NAFLD were histologically evaluated according to the criteria of Kleiner (NAFLD activity score) and the Brunt's criteria. At the time of the liver biopsy, blood was taken and levels of CatD, alanine aminotransferase (ALT) and cytokeratin-18 (CK-18) were measured in plasma.

\section{Results}

Plasma CatD levels were significantly lower in subjects with liver inflammation compared to steatotic subjects. Furthermore, we found that CatD levels were gradually reduced and corresponded with increasing severity of liver inflammation, steatosis, hepatocellular ballooning and NAFLD activity score. CatD levels correlated with pediatric NAFLD disease progression better than ALT and CK-18. In particular, CatD showed a high diagnostic accuracy (ROC-AUC: 0.94) for the differentiation between steatosis and hepatic inflammation, and reached almost the maximum accuracy (ROC-AUC: 0.998) upon the addition of CK-18.

\section{Conclusions}

Plasma CatD holds a high diagnostic value to distinguish pediatric patients with hepatic inflammation from children with steatosis. 


\section{Introduction}

The current obesity epidemic in children is paralleled by an increasing prevalence of non-alcoholic fatty liver disease (NAFLD), a condition characterized by early hepatic steatosis and by a more severe form known as non-alcoholic steatohepatitis (NASH). Key histologic components of NASH are steatosis, inflammation and hepatocellular ballooning. ${ }^{1}$ Children with biopsy-proven NAFLD were followed for 20 years and demonstrated a shorter long-term survival. Some children evolved towards advanced fibrosis, others to cirrhosis and a 20-year old girl needed a liver transplantation due to end-stage liver disease, hereby showing the progressive nature of NAFLD even in young individuals. ${ }^{2}$ Therefore, as NASH progresses to these more severe chronic liver conditions, its early detection is crucial to design adequate patient management and to reduce end-stage liver disease and overall mortality. Unfortunately, NASH cannot be identified without pursuing a liver biopsy, the reference standard for accurate staging and grading of NAFLD. Due to the invasiveness of this method, the high costs, the level of discomfort, sampling error and its risk for complications such as hemorrhage and bile peritonitis, ${ }^{3}$ liver biopsies, especially at a young age, should be replaced with non-invasive markers to detect NASH.

In addition to plasma alanine aminotransferase (ALT), a number of functionally diverse biomolecules could be used as potential markers for pediatric NASH, including several proteins that change their expression and stability during the development of steatosis, inflammation and ballooning. ${ }^{4}$ An extensively studied molecule is the caspase-cleaved cytokeratin-18 (CK-18), that, in addition to its ability to detect hepatocellular apoptosis, is also able to distinguish NASH from simple steatosis both in adults and children. ${ }^{5,6}$

Previously, we demonstrated a clear and direct association between hepatic inflammation and lysosomal cholesterol accumulation inside Kupffer cells (KCs) of low-density lipoprotein receptor knockout (Ldlr-/-) mice fed a high-fat, highcholesterol diet. $^{7-9}$ In line with our observation in mice, cholesterol-containing KCs were also demonstrated recently in livers of NASH patients. ${ }^{10}$ Further studies have shown that lysosomal cholesterol accumulation induces disturbances in lysosomal (enzyme) trafficking. ${ }^{11,12}$ In particular, cathepsins, the main class of lysosomal proteases, have been described to play an early role in inflammation. ${ }^{13}$ In line, recent evidence showed a disruption of hepatic cathepsin expression in NAFLD patients. ${ }^{14}$

We hypothesized that cathepsin D levels in plasma may be altered in subjects with NASH compared to subjects without NASH (i.e. steatosis). Therefore, the aim of the present study was to assess plasma levels of cathepsin $D$ in children with different stages of NAFLD and to correlate these to histological criteria used for the diagnosis of $\mathrm{NASH}$. Additionally, receiver operating characteristic (ROC) curves were plotted to test the diagnostic accuracy of cathepsin D. 


\section{Materials and methods}

Sample collection for the present study was performed at the Hepato-Metabolic Department of Bambino Gesù Children's Hospital during the period September 2012December 2013. The study design was conformed to the ethical guidelines of the Declaration of Helsinki (1975) and was performed according to the recommendations of the Ethics Committee of the Hospital.

We included consecutive children aged 3 to 12 years with an ultrasonographic diagnosis of NAFLD and persistently ( $\geq 6$ months) elevated serum aminotransferases. The decision to biopsy NAFLD children was based on our previously published data, demonstrating a high prevalence of necro-inflammatory changes and fibrosis in children with ultrasonographic steatosis and persistently elevated liver enzymes. ${ }^{15}$ Blood samples were obtained just before the ultrasound-guided liver biopsy. All blood samples were originally processed to yield plasma and stored at $-80^{\circ} \mathrm{C}$. Exclusion criteria were hepatic virus infections (hepatitis A, B, C, cytomegalovirus; and EpsteinBarr virus), type I diabetes mellitus, excessive alcohol consumption ( $\geq 140 \mathrm{~g} /$ week), history of parenteral nutrition, and use of drugs known to induce steatosis (eg, valproate, amiodarone, or prednisone) or to affect body weight and carbohydrate metabolism. Autoimmune liver disease, metabolic liver disease, Wilson's disease, celiac disease, type II diabetes mellitus and alpha-1-antitrypsin deficiency were ruled out using standard clinical, laboratory, and histological criteria. ${ }^{16,17}$

\section{Liver histology}

The clinical indication for liver biopsy was either to assess and better define the presence of NASH and the degree of fibrosis or to exclude other likely liver diseases. ${ }^{16}$ A Sonoline Omnia ultrasound machine (Siemens, Munich, Germany) equipped with a 5-MHz probe (5.0 C 50, Siemens) and a biopsy adaptor was employed.

The liver specimens of $\geq 15 \mathrm{~mm}$ length including at least 5-6 complete portal tracts were considered adequate for the purpose of the study. Biopsies were routinely processed (i.e. formalin-fixed and paraffin-embedded) and sections of liver tissue were stained with hematoxylin-eosin, Van Gieson, Periodic acid-Schiff diastase, and Prussian blue stain. Biopsies were evaluated by a single expert hepato-pathologist, who was unaware of the patient's clinical and laboratory data. To determine the intraobserver agreement, the pathologist scored the liver biopsies blindly twice and the weighted $\mathrm{k}$ coefficients for different histological features were calculated.

The main histological features of NAFLD/NASH, including steatosis, inflammation, hepatocellular ballooning and fibrosis were scored using the NAFLD Clinical Research Network (CRN) criteria. ${ }^{18}$ Briefly, steatosis was graded on a 4-point scale: grade $0=$ steatosis involving $<5 \%$ of hepatocytes; grade $1=$ steatosis involving up to $33 \%$ of hepatocytes; grade $2=$ steatosis involving $34-65 \%$ of hepatocytes; and grade $3=$ steatosis involving $>=66 \%$ of hepatocytes. Lobular inflammation was graded on a 
4-point scale: grade $0=$ no foci; grade $1=<2$ foci per $200 \times$ field; grade $2=2-4$ foci per 200xfield; and grade 3=>4 foci per 200x field. Hepatocellular ballooning was graded from 0 to 2: $0=$ none; $1=$ few balloon cells; and 2 =many/prominent balloon cells. The stage of fibrosis was quantified using a 5-point scale: stage $0=$ no fibrosis; stage $1=$ perisinusoidal or periportal ( $1 \mathrm{a}=$ mild, zone 3 , perisinusoidal; $1 \mathrm{~b}=$ moderate, zone 3 , perisinusoidal; 1c=portal/periportal); stage 2=perisinusoidal and portal/periportal; stage 3=bringing; and stage $4=$ cirrhosis. Features of steatosis, lobular inflammation, and hepatocellular ballooning were combined to obtain the NAFLD activity score (NAS score). The NAS score ranged from 1 to 7 . As recently recommended by NASH CRN ${ }^{19}$, a microscopic diagnosis based on overall injury pattern (steatosis, hepatocellular ballooning, inflammation) as well as the presence of additional lesions (e.g. zonality of lesions, portal inflammation and fibrosis) has been assigned to each case. Accordingly, biopsies were subdivided into: steatosis, borderline NASH and definite NASH. ${ }^{19}$

\section{Laboratory assessment}

At the time of the liver biopsy, blood was taken for further analysis. In all patients, enrolled in the present study, aspartate aminotransferase (AST), alanine aminotransferase (ALT), gamma-glutamyl transpeptidase (GGT), triglycerides, total cholesterol, albumin levels, glucose tolerance and prothrombin time (international normalized ratio, INR) were evaluated using standard laboratory methods. Insulin resistance was calculated according to the Homeostatic Model Assessment-Insulin Resistance (HOMA-IR) derived from basal values of glucose and insulin as previously described. ${ }^{20}$

\section{Human cathepsin D enzyme-linked immunosorbent assay}

Plasma samples were diluted and cathepsin $D$ levels were determined by the cathepsin D enzyme-linked immunosorbent assay according to the manufacturers' protocol (Uscn Life Science Inc, Wuhan, China). The absorbance was measured on a Benchmark 550 microplate reader (Bio-rad). The detection limit ranges approximately from 46.88 to $3,000 \mathrm{pg} / \mathrm{ml}$. Coefficients of variation (CV\%) for intra- and inter assays are $<10$ and $<12 \%$ respectively. Cathepsin D measurements were performed blinded to the histology findings of the study participants.

\section{CK-18 level measurements}

Part of plasma samples were used for quantitative determination of CK-18 levels by the M30-Apoptosense ELISA kit (PEVIVA) purchased from Li Starfish (Milan, Italy). All assays were performed in duplicates, and the absorbance was determined using a microplate reader (Molecular Bio-Rad, Milan, Italy). 


\section{Statistical analysis}

The data were analysed by performing one-way analysis of variance (ANOVA) and posthoc Tukey's test. The data were expressed as mean \pm SEM and considered significant at $p<0.05$. In order to evaluate the performance of plasma cathepsin $D$ for distinguishing steatosis vs. NASH patients, borderline NASH vs. NASH and, steatosis and borderline NASH vs. NASH, the sensitivity, specificity, predictive values and area under curve (AUC) were calculated using receiver operating characteristic (ROC) curve analysis. A statistical significance between two AUCs was evaluated by computing the $Z$-score, followed by calculating two-tailed p-values. $\mathrm{P}<0.05$ was considered significant. The analysis and the graphs were performed using GraphPad Prism 5 (version 5.03).

To assess the diagnostic accuracy of the combination CK-18 with cathepsin D, a logistic regression was fitted to steatosis, borderline NASH and NASH patients for both CK-18 and cathepsin D. Statistical ROC analyses and AUC presented for the combination CK18 with cathepsin $D$ were performed using the freely available program $R^{21}$ and the publicly available libraries 'gnlm' 22 and 'DiagnosisMed'.

\section{Results}

\section{General characteristics of the pediatric population}

Ninety-six children (mean age: 8.9 years, ranging from 3.3-12.1 years) with NAFLD were enrolled in the study. Of those ninety-six children, 56 were boys and 40 girls with a mean age of 9.3 and 8.4 years respectively. NASH was diagnosed in $27 \%$ (26 subjects) of the study population, while 51 subjects (53\%) were diagnosed with borderline NASH and 19 subjects (20\%) with steatosis (Table 6.1). All children were obese (BMI $>95^{\text {th }}$ percentile) and no difference was observed in BMI percentile and waist circumference (WC) percentile between children with steatosis, borderline NASH or definite NASH (Table 6.2). No differences were found in insulin resistance, glucose tolerance, the lipid profile and coagulation profile (HOMA-IR, impaired glucose tolerance (IGT), cholesterol, triglycerides, albumin, and INR). Liver-specific parameters including ALT, AST and GGT were similar between all the groups. In response to NAFLD severity, a gradual significant decrease was detected for plasma cathepsin D, a lysosomal protease (steatosis vs. borderline NASH: $p=0.0017$; steatosis vs. NASH: $\mathrm{p}<0.0001$; borderline NASH vs. NASH: $\mathrm{p}<0.0001)$. In contrast, CK-18 increased in response to NAFLD severity and could differentiate steatosis from borderline NASH ( $p=0.0001)$ and NASH $(p=0.0003)$. No difference in CK-18 levels was observed between borderline NASH and definite NASH $(p=0.95)$. 
Table 6.1 Histological features of the pediatric subjects.

\begin{tabular}{lccc}
\hline & $\begin{array}{c}\text { Steatosis } \\
(\mathrm{n}=19)\end{array}$ & $\begin{array}{c}\text { Borderline NASH } \\
(\mathrm{n}=51)\end{array}$ & $\begin{array}{c}\text { NASH } \\
(\mathrm{n}=26)\end{array}$ \\
\hline NAS & $2.2 \pm 0.3$ & $3.9 \pm 0.2$ & $5.7 \pm 0.1$ \\
Steatosis (\%) & $15(78.9)$ & $10(19.6)$ & $2(7.7)$ \\
$.5-33 \%$ & $3(15.8)$ & $5(9.8)$ & $5(19.2)$ \\
$.34-65 \%$ & $1(5.3)$ & $36(70.6)$ & $19(73.1)$ \\
.$>=66 \%$ & & & 0 \\
Inflammation (\%) & $5(26.3)$ & $1(2.0)$ & $12(46.2)$ \\
Grade 0 & $13(68.4)$ & $33(64.7)$ & $14(53.8)$ \\
Grade 1 & $1(5.3)$ & $17(33.3)$ & $3(11.5)$ \\
Grade 2 & & & $15(57.7)$ \\
Ballooning (\%) & $17(89.5)$ & $22(43.1)$ & $8(30.8)$ \\
. None & $1(5.3)$ & $24(47.1)$ & \\
. Few & $1(5.3)$ & $5(9.8)$ & $7(26.9)$ \\
. Many & & & $16(61.5)$ \\
Fibrosis (\%) & $10(52.6)$ & $19(37.3)$ & 0 \\
Stage 0 & $8(42.1)$ & $26(51.0)$ & $3(11.5)$ \\
Stage 1 & $1(5.3)$ & $5(9.8)$ & $1(2.0)$ \\
Stage 2 & 0 & & \\
Stage 3 & & & \\
\hline
\end{tabular}

NAS: NAFLD activity score. NAS is represented as mean \pm SEM.

Table 6.2 Clinical characteristics of the pediatric population.

\begin{tabular}{lcccccc}
\hline & Steatosis(1) & $\begin{array}{c}\text { Borderline } \\
\text { NASH(2) }\end{array}$ & NASH(3) & \multicolumn{2}{c}{ p-value between groups } \\
& $(\mathrm{n}=19)$ & $(\mathrm{n}=51)$ & $(\mathrm{n}=26)$ & 1 vs. 2 & 1 vs. 3 & 2 vs. 3 \\
\hline Male (\%) & $12(63.2)$ & $29(56.9)$ & $15(57.7)$ & 0.886 & 0.930 & 0.997 \\
Age (years) & $10.0 \pm 0.5$ & $8.4 \pm 0.3$ & $9.2 \pm 0.4$ & 0.016 & 0.378 & 0.314 \\
Weight (kg) & $52.0 \pm 2.6$ & $45.2 \pm 2.2$ & $44.3 \pm 2.0$ & 0.155 & 0.149 & 0.958 \\
Length (cm) & $139.9 \pm 2.8$ & $132.4 \pm 2.4$ & $135.0 \pm 2.7$ & 0.158 & 0.531 & 0.753 \\
BMI percentile & $96.0 \pm 0.8$ & $96.0 \pm 0.7$ & $96.1 \pm 0.8$ & 1.000 & 0.995 & 0.996 \\
WC percentile & $83.6 \pm 2.4$ & $77.9 \pm 1.4$ & $82.9 \pm 1.7$ & 0.079 & 0.973 & 0.084 \\
Obese (\%) & $19(100)$ & $47(92.2)$ & $24(92.3)$ & 0.458 & 0.551 & 1.000 \\
Cholesterol (mg/dl) & $167.3 \pm 8.6$ & $163.3 \pm 5.1$ & $153.3 \pm 7.6$ & 0.915 & 0.427 & 0.506 \\
Triglycerides (mg/dl) & $94.0 \pm 8.4$ & $111.1 \pm 8.2$ & $125.9 \pm 21.9$ & 0.664 & 0.328 & 0.685 \\
ALT(U/l) & $61.6 \pm 7.4$ & $83.7 \pm 5.6$ & $91.0 \pm 15.3$ & 0.258 & 0.152 & 0.830 \\
AST (U/l) & $46.7 \pm 3.5$ & $55.3 \pm 2.6$ & $57.4 \pm 7.2$ & 0.387 & 0.311 & 0.929 \\
GGT (U/l) & $26.0 \pm 2.5$ & $29.1 \pm 2.7$ & $30.0 \pm 4.4$ & 0.814 & 0.769 & 0.981 \\
Cathepsin D (pg/ml) & $32658 \pm 3640$ & $21428 \pm 1753$ & $8095 \pm 784$ & 0.002 & $<0.0001$ & $<0.0001$ \\
CK-18 (U/l) & $261.6 \pm 24.5$ & $350.6 \pm 11.2$ & $356.4 \pm 6.6$ & 0.0001 & 0.0003 & 0.950 \\
Albumin (g/dl) & $4.5 \pm 0.1$ & $4.5 \pm 0.1$ & $4.6 \pm 0.1$ & 0.866 & 0.668 & 0.869 \\
INR & $1.1 \pm 0.05$ & $1.2 \pm 0.03$ & $1.1 \pm 0.04$ & 0.838 & 0.997 & 0.853 \\
IGT(\%)/Prediabetes & $0(0)$ & $9(17.6)$ & $4(15.4)$ & 0.137 & 0.297 & 0.959 \\
HOMA-IR & $2.7 \pm 0.4$ & $2.5 \pm 0.3$ & $2.4 \pm 0.3$ & 0.973 & 0.922 & 0.970 \\
\hline
\end{tabular}

Data are represented as mean \pm SEM. Data was statistically analyzed by performing one-way ANOVA tests using SPSS software, version 22.0 (SPSS, Chicago, IL, USA). BMI: body mass index; WC: waist circumference; INR: international normalized ratio; CK-18: cytokeratin-18; IGT: impaired glucose tolerance; HOMA-IR: homeostasis model assessment of insulin resistance. 


\section{Significantly reduced levels of plasma cathepsin D correlate with pediatric NAFLD disease severity}

First, we investigated whether plasma cathepsin D levels correlated with histological features of NAFLD. We found that plasma levels of cathepsin D decreased significantly in parallel with the increasing degree of NAFLD and the NAS score (Figure 6.1). Furthermore, in line with the increased presence of hepatocellular ballooning, a key histological feature of NASH, plasma cathepsin D declined. By examining the individual stages of NAFLD, we demonstrate that, unlike late-stage fibrosis, cathepsin D was reduced upon increasing degrees of steatosis and inflammation. Thus, plasma cathepsin D was significantly lowered in children with NASH compared to children with either steatosis or borderline NASH and was associated with early stages of NAFLD (i.e. steatosis and inflammation).

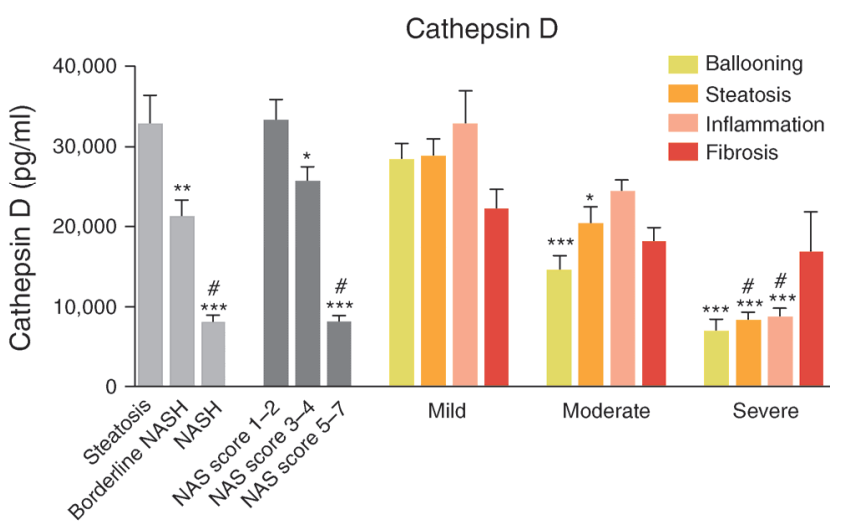

Figure 6.1 Cathepsin D in plasma of pediatric non-alcoholic fatty liver disease (NAFLD) subjects. Cathepsin D levels were analyzed in children with NAFLD divided by biopsy-proven steatosis, borderline non-alcoholic steatohepatitis (NASH), and NASH (light-grey bars) or divided upon the NAS score (dark-grey bars). The colored bars show plasma cathepsin D for the individual stages of NAFLD. Classification Mild indicates a stage 0 for hepatocellular ballooning, stage 1 for steatosis, and stage 0 for inflammation and fibrosis. Classification Moderate indicates stage 1 for hepatocellular ballooning, stage 2 for steatosis, and stage 1 for inflammation and fibrosis. Classification Severe indicates stage 2 for hepatocellular ballooning, stage 3 for steatosis, stage 2 for inflammation, and stage $2+3$ for fibrosis. Asterisks denote significance compared with the corresponding first (left) bar for each classification. Each classification is indicated by the light-grey, the dark-grey, and the colored bars. ${ }^{*} p<0.05,{ }^{* *} p<0.01$, and $* * * p<0.001$. The hashtag (\#) notation represents significance compared with the corresponding second (middle bar) for each classification. \# $p<0.001$. NAS, NAFLD activity score; NASH, non-alcoholic steatohepatitis. 


\section{Plasma ALT does not correlate with the NAFLD histological pattern in children}

Currently, plasma ALT is primarily used in the clinic as a non-invasive marker to detect NASH. In order to make a comparison between cathepsin D and ALT, plasma ALT levels were also measured. Opposite to cathepsin D, a trend towards increasing ALT levels in parallel with increasing NAFLD severity was found (Figure 6.2). However, this difference was not significant, neither for Brunt's or Kleiner's criteria. Unlike cathepsin $D, A L T$ levels remained similar upon severe degrees of hepatocellular ballooning and steatosis. ALT levels rose with increasing severity of inflammation, but did not reach a statistically significant level. During fibrosis, a trend towards an increase in ALT levels was found between patients who displayed no fibrosis and those who demonstrate mild or severe fibrosis. Altogether, ALT is not able to differentiate between the different stages of pediatric NAFLD and is more associated with late-stage fibrosis.

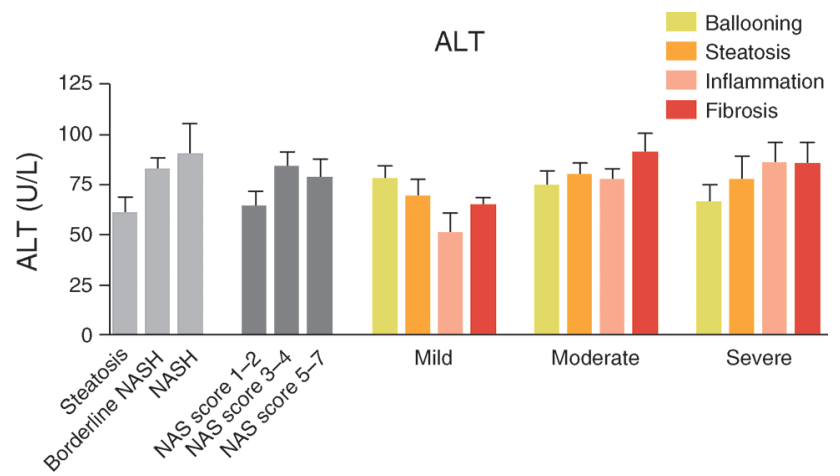

Figure 6.2 Plasma alanine aminotransferase (ALT) levels in children with biopsy-proven non-alcoholic fatty liver disease (NAFLD). ALT levels in children with NAFLD divided by biopsy-proven steatosis, borderline non-alcoholic steatohepatitis (NASH), and NASH (light-grey bars) or divided upon the NAS score (dark-grey bars). The colored bars show plasma ALT for the individual stages of NAFLD. NAS, NAFLD activity score; NASH, non-alcoholic steatohepatitis.

\section{CK-18 can differentiate between steatosis and NASH and correlates with some histological features of pediatric NAFLD}

As previous research has shown that plasma levels of CK-18 are accurate in predicting NASH vs. no NASH in adults ${ }^{5}$ and children ${ }^{6}$, we point to confirm these data in our cohort. In the current study, CK-18 was increased in pediatric subjects with borderline NASH and definite NASH compared to steatosis. However, CK-18 levels remained identical between children with borderline and definite NASH (Figure 6.3). CK-18 was significantly increased in subjects with a high NAS score (3-4 and 5-7) compared to children with an NAS score of 1-2. To determine whether there is an association 
between CK-18 and severity of NAFLD, we categorized CK-18 according to the histological stages of hepatocyte ballooning, steatosis, inflammation and fibrosis. In this cohort CK-18 was significantly increased upon mild hepatocyte ballooning and according to steatosis and inflammation severity. No difference in CK-18 levels between the different stages of fibrosis was observed. Altogether, we confirm that CK-18 is able to distinguish NASH from steatosis in children with NAFLD.

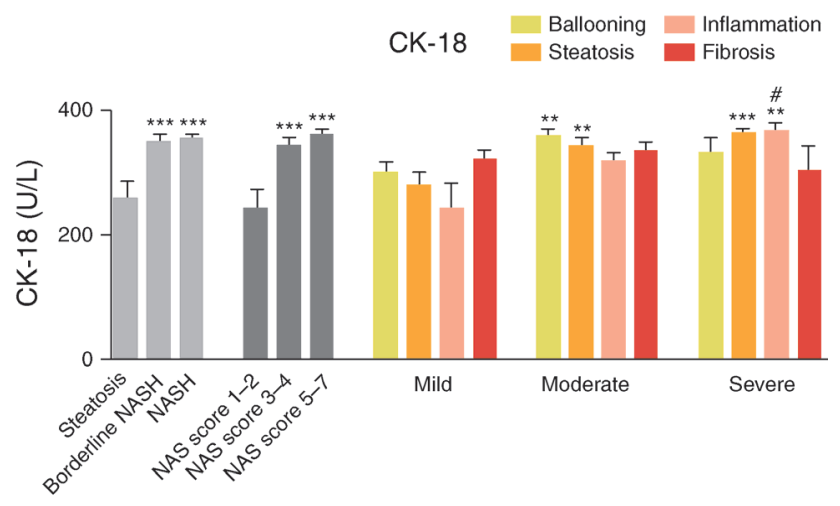

Figure 6.3 Cytokeratin-18 (CK-18) levels in plasma of children with non-alcoholic fatty liver disease (NAFLD). CK-18 measurements were plotted against the several stages of NAFLD. Asterisks denote significance compared with the corresponding first (left) bar for each classification. Each classification is indicated by the light-grey, the dark-grey, and the colored bars. ${ }^{*} \mathrm{p}<0.05$, $* * \mathrm{p}<0.01$, and $* * * \mathrm{p}<0.001$. The hashtag (\#) notation represents significance compared with the corresponding second (middle bar) for each classification. \# $\mathrm{P}<0.001$. NAS, NAFLD activity score; NASH, non-alcoholic steatohepatitis.

\section{Plasma cathepsin D holds a better predictive value for the differentiation between steatosis and NASH in children than CK-18 and ALT}

We next investigated the clinical potential of plasma cathepsin D for diagnosing pediatric NASH and hereby made use of plotted ROC curves. For the comparison between steatosis and NASH, the ROC curve of cathepsin D demonstrated a significant higher area under the curve (AUC) of 0.94, compared to the AUC values of ALT (0.59; $\mathrm{p}=0.0004)$ and CK-18 (0.72; $\mathrm{p}=0.0225)$ (Figure 6.4A and Table 6.3). Regarding the differentiation between borderline NASH and definite NASH, an AUC value of 0.85 was reached by using cathepsin $D$, which was significantly higher than the AUC values of $\operatorname{ALT}(0.57 ; p=0.0011)$ and CK-18 (0.57; $p=0.0003)$. An AUC value of 0.88 for cathepsin D was given for the differentiation between steatosis + borderline NASH vs. NASH, compared to an AUC of 0.52 and 0.53 for ALT and CK-18 respectively (Figure 6.4C). For this differentiation, further statistical analysis revealed that the AUC value of cathepsin D is higher than the AUC values of ALT $(p<0.0001)$ and CK-18 $(p<0.0001)$ 
(Table 6.3). No significant differences were observed between the AUCs of cathepsin D (AUC: 0.81), ALT (AUC: 0.66) and CK-18 (AUC: 0.74) to distinguish steatosis vs. borderline NASH + NASH (Figure 6.4D and Table 6.3).

A

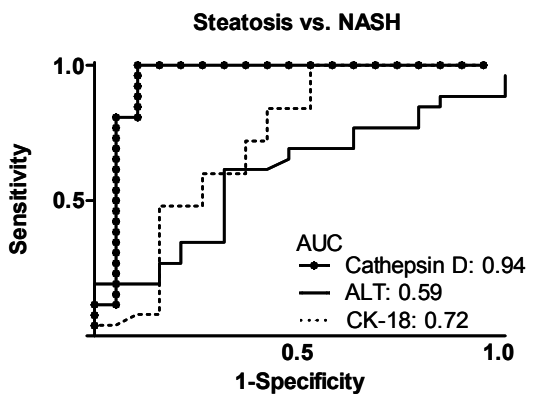

C

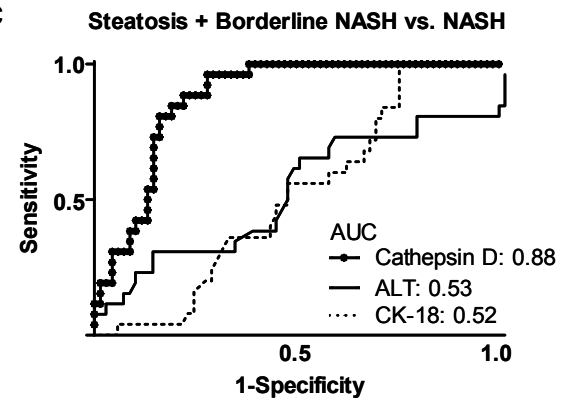

B

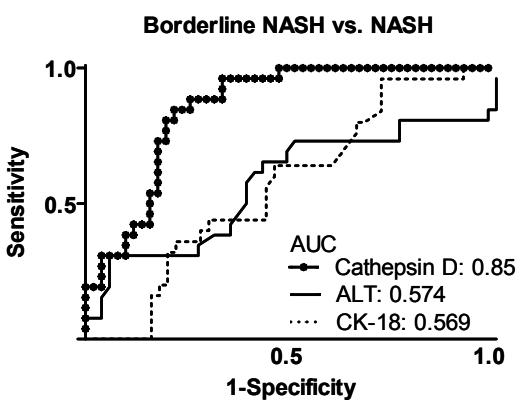

D

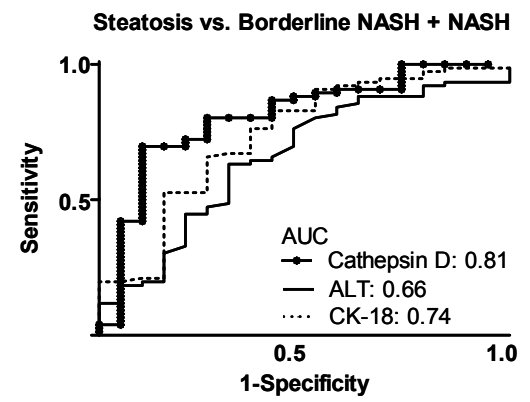

Figure 6.4 The diagnostic value of cathepsin D, CK-18 and ALT in pediatric NAFLD. ROC curve analysis was performed to assess the area under the curve (AUC) of using plasma cathepsin $D, C K-18$ or ALT to predict hepatic inflammation for the following comparisons; steatosis vs. NASH (A); borderline NASH vs. NASH (B); steatosis combined with borderline NASH vs. NASH (C) and steatosis vs. borderline NASH + NASH (D).

The diagnostic performances of cathepsin D, ALT and CK-18 in the prediction of NASH are depicted in Supplementary Table S6.1. The best cut-off point for cathepsin D was $<18445 \mathrm{pg} / \mathrm{ml}$, which demonstrated the highest sensitivity $(100 \%)$ and specificity (89.5\%). Positive and negative predictive values (PPV and NPV) were calculated and demonstrated $92.9 \%$ and $100 \%$ respectively. In contrast to cathepsin D, ALT and CK-18 displayed lower sensitivity and specificity rates and less accurate PPV and NPV percentages to predict pediatric NASH.

In short, compared to ALT and CK-18, cathepsin D holds better diagnostic value to predict pediatric NASH and is accurate in making the differentiation between pediatric NASH subjects from those who have steatosis. Similar to steatosis vs. NASH subjects, 
cathepsin D also improves the prediction of NASH compared to the combination of steatosis + borderline NASH, and also compared to borderline NASH separately.

Table 6.3 Overview of the calculated p-values between the different AUCs.

\begin{tabular}{lccc}
\hline & Cathepsin D vs. ALT & Cathepsin D vs. CK-18 & CK-18 vs. ALT \\
\hline Steatosis vs. NASH & $\mathrm{p}=0.0004$ & $\mathrm{p}=0.0225$ & $\mathrm{p}=0.2840$ \\
Borderline NASH vs. NASH & $\mathrm{p}=0.0011$ & $\mathrm{p}=0.0003$ & $\mathrm{p}=0.9629$ \\
Steatosis + Borderline NASH vs. NASH & $\mathrm{p}<0.0001$ & $\mathrm{p}<0.0001$ & $\mathrm{p}=0.9204$ \\
Steatosis vs. Borderline NASH + NASH & $\mathrm{p}=0.1030$ & $\mathrm{p}=0.4299$ & $\mathrm{p}=0.4189$ \\
\hline
\end{tabular}

P-values in bold indicate statistically significant differences.

\section{Adding CK-18 to cathepsin D improves the diagnostic accuracy to predict pediatric NASH}

To explore whether adding a liver-specific marker, CK-18, to cathepsin D would lead to a better prediction of pediatric NASH, we plotted ROC curves of cathepsin D combined with CK-18. Whereas cathepsin D alone demonstrated an AUC of 0.94 for the comparison of steatotic subjects vs. patients with NASH (Figure 6.4A), the combination CK-18 with cathepsins D resulted in an AUC of 0.998 (Figure 6.5A).

Compared to using cathepsin D alone, combining CK-18 with cathepsin D did not increase the AUC values for the differentiations borderline NASH vs. NASH, and steatosis + borderline NASH vs. NASH (Figure 6.4B-C; 6.5B-C). When distinguishing steatosis from borderline NASH + NASH patients by using cathepsin D individually, an AUC value of 0.81 was reached (Figure 6.4D). Upon the addition of CK-18, an AUC value of 0.85 was obtained (Figure 6.5D). These data illustrate that adding CK-18 to cathepsin $D$ contributes to reaching an optimal ROC curve for the diagnostic prediction of pediatric steatosis vs. NASH. 
A

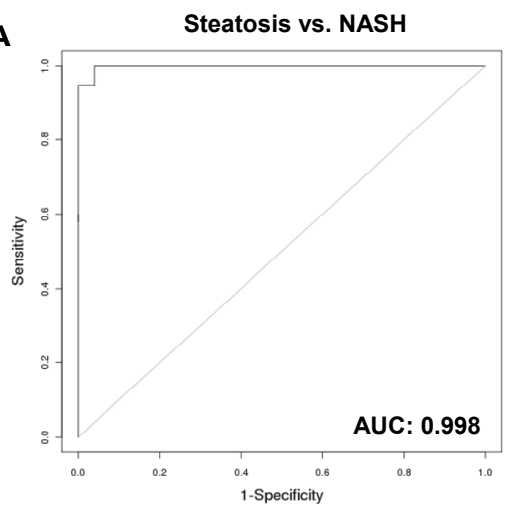

C Steatosis + Borderline NASH vs. NASH

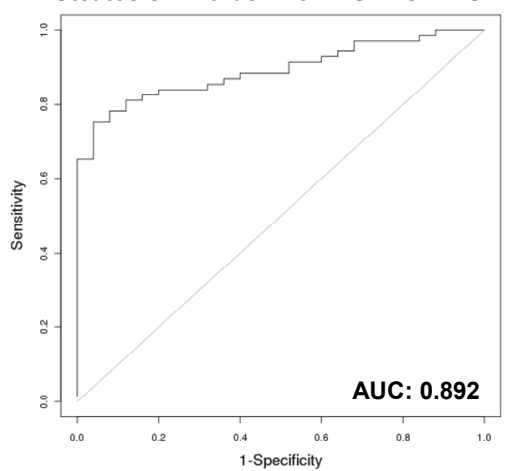

B

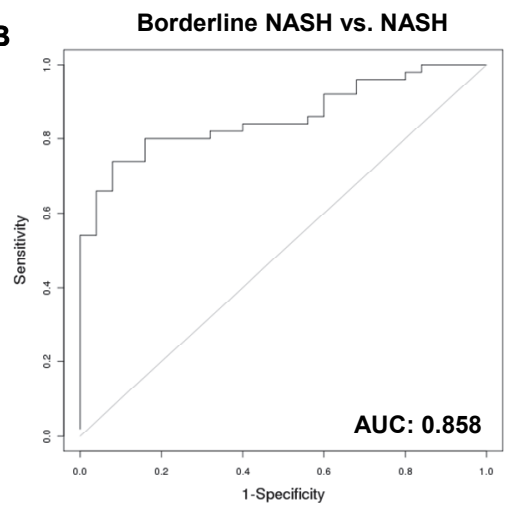

D Steatosis vs. Borderline NASH + NASH

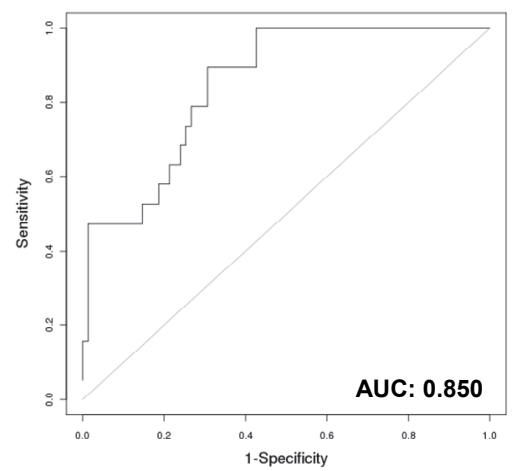

Figure 6.5 The diagnostic value of adding CK-18 to cathepsin D. ROC curve analysis was performed to assess the area under the curve (AUC) of using the combination of plasma CK-18 with cathepsin $D$ to predict hepatic inflammation for the following differentiations; steatosis $v s$. NASH (A); borderline NASH vs. NASH (B); steatosis combined with borderline NASH vs. NASH (C) and steatosis vs. borderline NASH + NASH (D).

\section{Discussion}

Diagnosing NASH during childhood is of critical importance in order to prevent further progression into NAFLD-related cirrhosis and end-stage liver disease during adolescence. Therefore, understanding the mechanisms that cause progression to $\mathrm{NASH}$ at an early age is crucial.

With the help of lysosomal enzymes, lysosomes are best known for its primary role in protein degradation. However, lysosomal function is not merely restricted to degradation of proteins, but increasing evidence now demonstrates that the lysosomal compartment plays also a role in the immune system and can be seen as 
secretory vesicles. ${ }^{23,24}$ Numerous studies have shown that lysosomal cholesterol accumulation inside macrophages is an event that occurs during inflammation and has been detected in NASH as well as atherosclerosis. ${ }^{7,25}$ In line, cathepsins have been shown to be significantly involved in mediating the inflammatory response and cholesterol trafficking. ${ }^{13,26,27}$

Furthermore, it has been reported that cathepsin expression was impaired in the liver of NAFLD patients, suggesting a pivotal role of these proteins in the setting of liver inflammation that characterize the disease. ${ }^{14}$ In addition, these findings would indicate that cathepsins are more likely to be involved during early stages of NAFLD, rather than a reflection of a late consequence of inflammation. Indeed, in the current study we showed that levels of plasma cathepsin D are decreased at early stages of $\mathrm{NASH}$, while these remain similar upon the different stages of fibrosis. This was in contrast to plasma ALT, which showed an increase during late-stage fibrosis and was more representative for liver damage. Likewise, a correlation exists between circulating cathepsin $D$ and carotid intima-media thickness, an indicator for atherosclerosis. ${ }^{28}$ In line with these results, abnormal cathepsin D fractions were associated with a lysosomal storage disease ${ }^{29}$, a disease predominantly characterized by chronic systemic inflammation. Thus, it seems that lysosomal cholesterol accumulation in KCs, and its subsequent effect on lysosomal enzyme homeostasis, plays a central role in the development of childhood NASH. While many evidences point to the link between lysosomal enzymes and $\mathrm{NASH}$, the exact mechanisms that lead to the secretion of the lysosomal content in plasma are not yet known.

Lysosomes are able to secrete its content, including lysosomal enzymes, via fusion with the plasma membrane. Such fusion is a calcium-dependent process and has been shown to be sensitive for cholesterol levels. As a result, inducing lysosomal cholesterol accumulation completely inhibited lysosomal exocytosis, suggesting that storage of lysosomal cholesterol plays a crucial role in pediatric NASH. ${ }^{30}$ These data are in line with previous results showing high cholesterol levels in $\mathrm{KCS}$, as was indicated by numerous cholesterol-filled droplets in KCs of livers of NASH patients, while livers of steatotic patients did not show this typical foam cell-like phenotype. ${ }^{10}$ However, foam-cell like structures were so far only detected in NASH livers of adults, and still needs to be confirmed in NASH livers of children.

Excessive cholesterol inside lysosomes can also damage the lysosomal membrane and cause lysosomal membrane permeabilization. In turn, there is leakage of cathepsins into the cytosol, whereby cathepsins can mediate the apoptotic signaling pathway. ${ }^{31}$ Thus, instead of extracellular secretion, less viable cells remain left to secrete cathepsin D due to increased apoptosis.

The gold standard to diagnose pediatric NASH is still an invasive liver biopsy procedure. However, due to its invasiveness, biopsies are not performed as a first step. Therefore, clinicians rely on several non-invasive methods to detect NASH in children such as, liver markers (ALT/AST) and imaging techniques (ultrasound, CT scan 
and MRI). Currently, ALT is the primary clinical parameter to evaluate NAFLD in children. However, by using a biopsy-confirmed NAFLD cohort it was found that AST and ALT have limited predictive power to discriminate between low and high NAS scores in children with AUC values varying from 0.59 to $0.76 .^{32}$ In line, normal ALT levels were observed despite the presence of NAFLD, confirming a low correlation between ALT and NAFLD. ${ }^{33}$ These data are confirmed in our study by demonstrating low AUC (0.53-0.59) and predictive values (PPV: 72.7\% and NPV: 56.5\%) for ALT in the discrimination between NASH and no NASH.

A promising serum marker in adults is CK-18, which is representative for hepatocyte apoptosis. Recently, CK-18 was tested in a biopsy-proven children cohort, which confirmed that CK-18 is also an accurate way of predicting NASH vs. steatosis already during early life. ${ }^{34}$ Similarly, a second pediatric study confirmed the high diagnostic accuracy for CK-18 in predicting NASH (AUC value: 0.93 ) and a PPV and NPV of $94 \%$ and $72 \%$, respectively. ${ }^{6}$ Comparable to these studies, we now found a high AUC value (0.72) and similar PPV and NPV values of CK-18 (72 and 100\% respectively) for the detection of NASH vs. steatosis, confirming that CK-18 is accurate at diagnosing NASH in children. However, in our cohort, plasma cathepsin D correlated better with pediatric NAFLD disease progression than CK-18. Altogether, in contrast to other plasma parameters tested for pediatric NASH, our data suggest that plasma cathepsin $D$ is a highly accurate and early non-invasive marker to distinguish steatosis vs. NASH in children. In contrast to CK-18, cathepsin D correlated significantly with NAFLD severity.

Despite our significant findings, the cohort that was used is relatively small in patient size and is of cross-sectional nature. Additional cohorts and future longitudinal studies are essential to validate plasma cathepsin D as a useful tool to detect pediatric hepatic inflammation. Moreover, in view of statistics, a larger sample size would also boost the statistical power. Furthermore, intervention studies should be performed to test whether plasma cathepsin D could be implicated in the clinical follow-up. As obtaining liver biopsies from children with normal aminotransferases is rarely performed, all pediatric individuals in the current study demonstrate abnormal aminotransferase levels. As such, children with normal levels of aminotransferases are not included and consequently, the level of plasma cathepsin D under healthy conditions is not known. The same restriction holds true for studying cathepsin $D$ in relation to NAFLD in children or adults with a normal body mass index.

Altogether, in this study, we used a well-defined biopsy-proven population of children and evaluated plasma levels of cathepsin D. We have demonstrated for the first time that plasma cathepsin D is significantly decreased in children with NASH compared to children with either steatosis or borderline NASH. Plasma cathepsin D holds high diagnostic value and could be used as a promising non-invasive clinical marker to distinguish pediatric NASH patients from subjects with steatosis, especially upon the 
addition of CK-18. Furthermore, this is the first human study to show that the lysosomal compartment changes under inflammatory conditions. However, future research is warranted to fully understand the novel role of lysosomes during the development of early NASH. Moreover, whether cathepsin D can be used in clinical practice as a non-invasive marker for NASH, should be validated in additional larger well-defined NAFLD cohorts in children and adults. 


\section{References}

1. Alisi A, Manco M, Vania A, et al. Pediatric nonalcoholic fatty liver disease in 2009. J Pediatr 2009;155:469-74.

2. Feldstein $A E$, Charatcharoenwitthaya $P$, Treeprasertsuk $S$, et al. The natural history of non-alcoholic fatty liver disease in children: a follow-up study for up to 20 years. Gut 2009;58:1538-44.

3. Tobkes Al, Nord HJ. Liver biopsy: review of methodology and complications. Dig Dis 1995;13:267-74.

4. Nobili V, Svegliati-Baroni G, Alisi A, et al. A 360-degree overview of paediatric NAFLD: recent insights. J Hepatol 2013;58:1218-29.

5. Feldstein AE, Wieckowska A, Lopez AR, et al. Cytokeratin-18 fragment levels as noninvasive biomarkers for nonalcoholic steatohepatitis: a multicenter validation study. Hepatology 2009;50:1072-8.

6. Feldstein $A E$, Alkhouri N, De Vito R, et al. Serum cytokeratin-18 fragment levels are useful biomarkers for nonalcoholic steatohepatitis in children. Am J Gastroenterol 2013;108:1526-31.

7. Bieghs V, Hendrikx T, van Gorp PJ, et al. The cholesterol derivative 27-hydroxycholesterol reduces steatohepatitis in mice. Gastroenterology 2013;144:167-178 e1.

8. Bieghs V, van Gorp PJ, Walenbergh SM, et al. Specific immunization strategies against oxidized lowdensity lipoprotein: a novel way to reduce nonalcoholic steatohepatitis in mice. Hepatology 2012;56: 894-903.

9. Bieghs V, Verheyen F, van Gorp PJ, et al. Internalization of modified lipids by CD36 and SR-A leads to hepatic inflammation and lysosomal cholesterol storage in Kupffer cells. PLoS One 2012;7:e34378.

10. Ioannou GN, Haigh WG, Thorning D, et al. Hepatic cholesterol crystals and crown-like structures distinguish NASH from simple steatosis. J Lipid Res 2013;54:1326-34.

11. Kornfeld S. Trafficking of lysosomal enzymes in normal and disease states. J Clin Invest 1986;77:1-6.

12. Shen $D$, Wang $X$, Li X, et al. Lipid storage disorders block lysosomal trafficking by inhibiting a TRP channel and lysosomal calcium release. Nat Commun 2012;3:731.

13. Decock J, Obermajer N, Vozelj S, et al. Cathepsin B, cathepsin H, cathepsin X and cystatin C in sera of patients with early-stage and inflammatory breast cancer. Int J Biol Markers 2008;23:161-8.

14. Fukuo $\mathrm{Y}$, Yamashina $\mathrm{S}$, Sonoue $\mathrm{H}$, et al. Abnormality of autophagic function and cathepsin expression in the liver from patients with non-alcoholic fatty liver disease. Hepatol Res 2014.

15. Manco M, Alisi A, Nobili V. Risk of severe liver disease in NAFLD with normal ALT levels: a pediatric report. Hepatology 2008;48:2087-8; author reply 2088.

16. Vajro $P$, Lenta $S$, Socha $P$, et al. Diagnosis of nonalcoholic fatty liver disease in children and adolescents: position paper of the ESPGHAN Hepatology Committee. J Pediatr Gastroenterol Nutr 2012;54:700-13.

17. American Diabetes A. Standards of medical care in diabetes--2014. Diabetes Care 2014;37 Suppl 1:S14-80.

18. Kleiner DE, Brunt EM, Van Natta M, et al. Design and validation of a histological scoring system for nonalcoholic fatty liver disease. Hepatology 2005;41:1313-21.

19. Brunt EM, Kleiner DE, Wilson LA, et al. Nonalcoholic fatty liver disease (NAFLD) activity score and the histopathologic diagnosis in NAFLD: distinct clinicopathologic meanings. Hepatology 2011;53:810-20.

20. Matthews DR, Hosker JP, Rudenski AS, et al. Homeostasis model assessment: insulin resistance and beta-cell function from fasting plasma glucose and insulin concentrations in man. Diabetologia 1985; 28:412-9.

21. Ihaka R, Gentleman R. A language for data analysis and graphics. Journal of Computational Graphics and Statistics 1996;5:299-314.

22. Lindsey JK. Models for repeated measurements. Second ed. Oxford: Oxford University Press; 1999.

23. Bordon Y. Immune regulation: lysosomes at the heart of inflammation. Nat Rev Immunol 2011; 11:502.

24. Samie MA, Xu H. Lysosomal Exocytosis and Lipid Storage Disorders. J Lipid Res 2014.

25. Bieghs $\mathrm{V}$, Rensen PC, Hofker $\mathrm{MH}$, et al. NASH and atherosclerosis are two aspects of a shared disease: central role for macrophages. Atherosclerosis 2012;220:287-93.

26. Hannaford J, Guo H, Chen X. Involvement of cathepsins B and L in inflammation and cholesterol trafficking protein NPC2 secretion in macrophages. Obesity (Silver Spring) 2013;21:1586-95. 
27. Sukhova GK, Zhang Y, Pan JH, et al. Deficiency of cathepsin S reduces atherosclerosis in LDL receptordeficient mice. J Clin Invest 2003;111:897-906.

28. Moallem SA, Nazemian F, Eliasi S, et al. Correlation between cathepsin D serum concentration and carotid intima-media thickness in hemodialysis patients. Int Urol Nephrol 2011;43:841-8.

29. Amritraj A, Peake K, Kodam A, et al. Increased activity and altered subcellular distribution of lysosomal enzymes determine neuronal vulnerability in Niemann-Pick type C1-deficient mice. Am J Pathol 2009;175:2540-56.

30. Xu J, Toops KA, Diaz F, et al. Mechanism of polarized lysosome exocytosis in epithelial cells. J Cell Sci 2012;125:5937-43.

31. Roberg K, Johansson $\mathrm{U}$, Ollinger K. Lysosomal release of cathepsin D precedes relocation of cytochrome $\mathrm{c}$ and loss of mitochondrial transmembrane potential during apoptosis induced by oxidative stress. Free Radic Biol Med 1999;27:1228-37.

32. Patton HM, Lavine JE, Van Natta ML, et al. Clinical correlates of histopathology in pediatric nonalcoholic steatohepatitis. Gastroenterology 2008;135:1961-1971 e2.

33. Mofrad $\mathrm{P}$, Contos MJ, Haque $\mathrm{M}$, et al. Clinical and histologic spectrum of nonalcoholic fatty liver disease associated with normal ALT values. Hepatology 2003;37:1286-92.

34. Fitzpatrick E, Mitry RR, Quaglia A, et al. Serum levels of CK18 M30 and leptin are useful predictors of steatohepatitis and fibrosis in paediatric NAFLD. J Pediatr Gastroenterol Nutr 2010;51:500-6. 


\section{Supplementary table}

Table S6.1 Different cut-off values of cathepsin D, CK-18 and ALT for distinguishing pediatric NASH from steatosis.

\begin{tabular}{ccccc}
\hline Cathepsin D (pg/ml) & Sensitivity $\%$ & Specificity $\%$ & PPV \% & NPV \% \\
\hline$<19811$ & 100.0 & 79.0 & 86.7 & 100.0 \\
$<19070$ & 100.0 & 84.2 & 89.6 & 100.0 \\
$<18445$ & 100.0 & 89.5 & 92.9 & 100.0 \\
$<16471$ & 96.2 & 89.5 & 92.6 & 94.4 \\
$<14612$ & 92.3 & 89.5 & 92.3 & 89.5 \\
$<13172$ & 88.5 & 89.5 & 92.0 & 85.0 \\
CK-18 (U/L) & Sensitivity $\%$ & Specificity \% & PPV \% & NPV \% \\
$>$ >257.0 & 100.0 & 47.4 & 72.2 & 100.0 \\
$>318.0$ & 84.0 & 57.9 & 73.7 & 72.6 \\
$>327.5$ & 72.0 & 63.2 & 72.8 & 62.2 \\
$>345.5$ & 60.0 & 73.7 & 75.7 & 57.4 \\
$>364.5$ & 48.0 & 84.2 & 80.6 & 54.2 \\
$>396.5$ & 8.0 & 89.5 & 51.0 & 42.2 \\
$>411.5$ & 4.0 & 100.0 & 100.0 & 43.2 \\
ALT (U/L) & Senifity $\%$ & PPV \% & NPV \% \\
$>34.0$ & 84.6 & 21.1 & 59.5 & 50.0 \\
$>42.0$ & 76.9 & 36.8 & 62.5 & 53.8 \\
$>54.5$ & 69.2 & 52.6 & 66.7 & 55.6 \\
$>$ >64.5 & 61.5 & 68.4 & 72.7 & 56.5 \\
$>76.5$ & 34.6 & 79.0 & 69.2 & 46.9 \\
$>87.0$ & 26.9 & 84.2 & 70.0 & 45.7 \\
$>169.0$ & 19.2 & 100.0 & 100.0 & 47.5 \\
\hline
\end{tabular}

PPV: positive predictive value, NPV: negative predictive value. 
Chapter 6 


\section{Chapter 7}

\section{Plasma cathepsin D correlates with histological classifications of fatty liver disease in adults and responds to intervention}

SMA Walenbergh, T Houben, SS Rensen, V Bieghs, T Hendrikx, PJ van Gorp, MLJ Jeurissen, WA Buurman, JWM Greve, J Plat, MH Hofker, S Kalhan, J Pihlajamäki, PJ Lindsey, GH Koek, R Shiri-Sverdlov

Submitted 


\section{Abstract}

\section{Background and aims}

Non-alcoholic steatohepatitis (NASH) is characterized by liver lipid accumulation and inflammation. The mechanisms that trigger hepatic inflammation are poorly understood and subsequently, no specific non-invasive markers exist. We have previously demonstrated a reduction in the plasma lysosomal enzyme, cathepsin D (CatD), in children with NASH compared to children without NASH. Recent studies have raised the concept that NAFLD in adults is distinct than children due to differences in the histological pattern in the liver. So far, the link between plasma CatD to the development of adult NASH was not examined. In the current manuscript, we investigated whether plasma CatD in adults correlates with NASH development and regression.

\section{Methods}

Biopsies were histologically evaluated for inflammation and NAFLD in three complementary cohorts of adults (total $n=248$ ). CatD and alanine aminotransferase (ALT) were measured in plasma.

\section{Results}

In contrast to our previous observations with childhood NASH, in all three adult NASH cohorts, we observed increased levels of plasma CatD in patients with NASH compared to adults without liver inflammation. Furthermore, after surgical intervention, we found a significant reduction of plasma CatD levels compared to the initial levels.

\section{Conclusions}

Our observations highlight a distinct pathophysiology between NASH in children and adults. The observation that CatD levels were correlated with NASH development and regression is promising. Additional studies in bigger NASH cohorts are warranted to explore the potential of plasma CatD as a novel non-invasive marker for NASH. 


\section{Introduction}

The current obesity epidemic is paralleled by an increasing prevalence of nonalcoholic steatohepatitis (NASH). NASH is characterized by hepatic lipid accumulation (steatosis) and inflammation. While steatosis itself is generally considered benign and reversible, the presence of inflammation will eventually set the stage for further liver damage, including fibrosis, cirrhosis and liver cancer. ${ }^{1,2}$ Currently, an elevation in the level of plasma alanine transaminase (ALT), a liver enzyme, is the primary clinical abnormality detected in NASH patients. When hepatocellular injury is present, this liver enzyme is released into the circulation. Although ALT is used as a tool for the routine diagnosis of $\mathrm{NASH}$, it lacks specificity and sensitivity to distinguish between NASH and steatosis. ${ }^{3,4}$ In order to improve non-invasive diagnosis of NASH, it is of clinical relevance to investigate novel underlying mechanisms during NASH pathophysiology. Although several processes have been identified that participate in the development of liver inflammation, the actual mechanisms for the inflammatory response remain uncertain.

We previously demonstrated a clear and direct association between hepatic inflammation and lysosomal cholesterol accumulation inside Kupffer cells (KCs) of low-density lipoprotein receptor knockout $\left(\mathrm{Ldll}^{-/-}\right)$mice fed a high-fat, high-cholesterol diet. $^{5-7}$ In accordance with our observation in mice, cholesterol-containing KCs were also demonstrated recently in livers of NASH patients. ${ }^{8}$ The occurrence of lysosomal cholesterol accumulation has been shown to induce disturbances in the lysosomal (enzyme) trafficking pathway. ${ }^{9,10}$ In line, we previously detected modified levels of the lysosomal enzyme, cathepsin D (CatD), in the plasma of children with NASH compared to children without hepatic inflammation. ${ }^{11}$

Recent studies have raised the concept that NAFLD in children and adults is distinct due to differences in the histological pattern in the liver as well as pathological characteristics of the disease. ${ }^{12-15}$ Thus far, the relationship between plasma CatD and adult NASH has not been explored. Therefore, as a follow-up study, we investigated in the current paper whether plasma CatD in adults correlates with the development and regression of NASH.

Here, we observed in three complementary cohorts of adults that plasma CatD is significantly increased along the development of NASH. As the plasma CatD levels in adults with NASH were the opposite of those previously found in children with $\mathrm{NASH}^{11}$, we point toward the existence of a potential different pathophysiology between NASH in children and adults. Our data further show that CatD responds to intervention, and underlines the potential clinical relevance and diagnostic value of plasma CatD in the context of NASH. 


\section{Materials and methods}

\section{Human cohorts; Cleveland cohort, Maastricht cohort and the Kuopio cohort}

The design of this study includes three cohorts; the Cleveland cohort consists of NASH patients from which biopsies were obtained and histologically evaluated for inflammation after the detection of abnormal plasma ALT values. The second and third population (the Maastricht and Kuopio cohort, respectively) include morbidly obese patients that are unselected for ALT, i.e. biopsies were taken during bariatric surgery regardless of their ALT levels. Subsequently, liver biopsies were histologically graded for NASH. Patients from these cohorts are considered to have a wide spectrum of fatty liver diseases, including early stages.

\section{Definition Cleveland cohort}

A total of 127 individuals (average body mass index (BMI) of $30.4 \mathrm{~kg} / \mathrm{m}^{2}$ ) were included, of which 43 had biopsy-proven NASH. Subjects with non-alcoholic fatty liver disease (NAFLD) were recruited from the metabolic clinics of the Cleveland Clinic and MetroHealth Medical Center in Cleveland, $\mathrm{OH}$. Excluding criteria, informed consent, approval, the procedure of obtaining and scoring of liver biopsies as well as blood sampling of this NASH Cleveland cohort have been described previously. ${ }^{16-18}$ See Table 7.1 for the patient characteristics of the Cleveland cohort.

Table 7.1 Population characteristics of the Cleveland cohort.

\begin{tabular}{lcc}
\hline & \multicolumn{2}{c}{ Cleveland cohort } \\
\cline { 2 - 3 } & No inflammation & Inflammation \\
\hline $\mathrm{n}$ & 84 & 43 \\
Sex, male/female & $35 / 49$ & $17 / 26$ \\
$\mathrm{Age}, \mathrm{y}$ & $41.8 \pm 1.3$ & $45.8 \pm 1.8$ \\
$\mathrm{BMI}, \mathrm{kg} / \mathrm{m}^{2}$ & $28.2 \pm 0.7$ & $34.8 \pm 0.8^{* * *}$ \\
Total cholesterol, mmol/l & $4.7 \pm 0.1$ & $5.0 \pm 0.2$ \\
$\mathrm{HDL}, \mathrm{mmol} / \mathrm{l}$ & $1.3 \pm 0.1$ & $1.1 \pm 0.04^{*}$ \\
LDL, mmol/l & $2.8 \pm 0.1$ & $3.2 \pm 0.1^{*}$ \\
Triglycerides, mmol/l & $1.3 \pm 0.1$ & $1.8 \pm 0.1^{*}$ \\
ALT, IU/l & $24.1 \pm 2.1$ & $77.0 \pm 8.5^{* * *}$ \\
AST, IU/l & $24.2 \pm 1.0$ & $60.2 \pm 6.3^{* * *}$ \\
AST/ALT ratio & $1.2 \pm 0.1$ & $0.9 \pm 0.1^{* * *}$ \\
\hline
\end{tabular}

Data are represented as mean \pm SEM. Significantly different from No inflammation, * and *** indicates $p<0.05$ and $p<0.001$, respectively.

\section{Definition Maastricht cohort}

Forty-seven severely obese patients, with an average BMI of $46.7 \mathrm{~kg} / \mathrm{m}^{2}$, undergoing bariatric surgery at the Maastricht University Medical Center or at the Atrium Medical 
Center Parkstad, Heerlen, the Netherlands, were included in the study. Excluding criteria, informed consent, the procedure of obtaining liver biopsies and blood sampling of this Maastricht morbidly obese cohort has been described previously. ${ }^{19,20}$ See Table 7.2 for the population characteristics of the Maastricht cohort. Liver biopsies were evaluated by a trained pathologist, according to the criteria of Brunt ${ }^{21}$ and Kleiner $^{18}$ and graded for severity from NASH grade 1 (early-stage), to NASH grade 3 (late-stage) (Table S7.1).

Table 7.2 Population characteristics of the Maastricht cohort.

\begin{tabular}{lccc}
\hline & \multicolumn{3}{c}{ Maastricht cohort } \\
\cline { 2 - 4 } & Normal & Steatosis & NASH \\
\hline $\mathrm{n}$ & 12 & 8 & 27 \\
Sex, male/female & $5 / 12$ & $0 / 8$ & $8 / 19$ \\
Age, $\mathrm{y}$ & $40.5 \pm 2.3$ & $40.0 \pm 3.9$ & $48.5 \pm 2.0^{* \prime} \#$ \\
$\mathrm{BMI}, \mathrm{kg} / \mathrm{m}^{2}$ & $43.5 \pm 2.0$ & $44.6 \pm 2.5$ & $48.8 \pm 1.8$ \\
Total cholesterol, $\mathrm{mmol} / \mathrm{l}$ & $4.9 \pm 0.3$ & $5.4 \pm 0.3$ & $5.5 \pm 0.3$ \\
$\mathrm{HDL}, \mathrm{mmol} / \mathrm{l}$ & $0.9 \pm 0.1$ & $1.0 \pm 0.1$ & $1.0 \pm 0.1$ \\
LDL, mmol/l & $3.1 \pm 0.2$ & $3.4 \pm 0.4$ & $3.5 \pm 0.2$ \\
Triglycerides, mmol/l & $1.8 \pm 0.2$ & $2.2 \pm 0.4$ & $2.8 \pm 0.6$ \\
ALT, IU/l & $18.2 \pm 1.1$ & $28.7 \pm 5.1^{*}$ & $29.0 \pm 2.9^{*}$ \\
AST, IU/l & $18.3 \pm 2.2$ & $22.4 \pm 2.8$ & $29.5 \pm 2.6^{*}$ \\
AST/ALT ratio & $1.1 \pm 0.2$ & $0.8 \pm 0.1$ & $1.1 \pm 0.1$ \\
\hline
\end{tabular}

Data are represented as mean \pm SEM. ${ }^{*}$ and \# indicate $p<0.05$. Asteriks $\left({ }^{*}\right)$ indicate significant changes compared with Normal. Hashtag (\#) indicates significant changes compared with Steatosis.

\section{Definition Kuopio cohort}

All patients undergoing obesity surgery in Kuopio University Hospital are recruited into our ongoing study investigating metabolic consequences of obesity surgery (Kuopio Obesity Surgery Study). ${ }^{22,23}$ The study group included 74 consecutive subjects, with an average BMI of $44.0 \mathrm{~kg} / \mathrm{m}^{2}$, who were accepted for Roux-en-Y gastric bypass (RYGB) operation over the years 2005-2010. See Table 7.3 for the general population characteristics at the start of intervention. The study protocol has been approved by the Ethics Committee of the Northern Savo Hospital District (54/2005, 104/2008 and 27/2010), and it was performed in accordance with the Helsinki Declaration. Written informed consent was obtained from the subjects. All patients with alcohol consumption $>2$ doses per day were excluded from the study. Chronic hepatitis B and $C$ were excluded using serology if ALT values were elevated prior to surgery (HCV and HBV infections are rare in Finland). Hemochromatosis was excluded by histological analysis of liver biopsies, and by normal serum ferritin levels in subjects that had elevated serum ALT level. Blood samples were taken after 12 hour fasting before the obesity surgery and 12 months after the surgery. Plasma lipids and aminotransferases were analyzed as described before. ${ }^{22,23}$ Liver biopsies were obtained using Trucut needle (Radiplast AB, Uppsala, Sweden) or as a wedge biopsy during elective gastric 
bypass operation. Overall histological assessment of liver biopsy samples was performed by one pathologist according to the standard criteria for scoring NAFLD. ${ }^{24}$

Table 7.3 Population characteristics of the Kuopio cohort

\begin{tabular}{lccc}
\hline & \multicolumn{3}{c}{ Kuopio cohort } \\
\cline { 2 - 4 } & Normal & Steatosis & NASH \\
\hline $\mathrm{n}$ & 29 & 23 & 22 \\
Sex, male/female & $8 / 21$ & $3 / 20$ & $10 / 12$ \\
$\mathrm{Age}, \mathrm{y}$ & $50 \pm 1.7$ & $46 \pm 2.0$ & $47 \pm 2.0$ \\
$\mathrm{BMI}, \mathrm{kg} / \mathrm{m}^{2}$ & $42.8 \pm 1.2$ & $44.6 \pm 1.0$ & $44.7 \pm 1.3$ \\
Total cholesterol, mmol/l & $4.1 \pm 0.1$ & $4.0 \pm 0.2$ & $5.0 \pm 0.3^{* *}, \#$ \\
$\mathrm{HDL}, \mathrm{mmol} / \mathrm{l}$ & $1.1 \pm 0.05$ & $1.0 \pm 0.04$ & $1.1 \pm 0.09$ \\
LDL, mmol/l & $2.3 \pm 0.1$ & $2.3 \pm 0.2$ & $3.0 \pm 0.2^{* *}, \# \#$ \\
Triglycerides, mmol/l & $1.4 \pm 0.1$ & $1.6 \pm 0.1$ & $1.9 \pm 0.2^{*}$ \\
ALT, IU/l & $36.9 \pm 5.0$ & $42.7 \pm 3.8$ & $64.1 \pm 10.6^{*}$ \\
AST, IU/l & $28.6 \pm 2.8$ & $27.1 \pm 1.7$ & $46.8 \pm 8.4^{*}, \#$ \\
AST/ALT ratio & $0.8 \pm 0.07$ & $0.7 \pm 0.03$ & $0.8 \pm 0.1$ \\
\hline
\end{tabular}

Data are represented as mean \pm SEM. Similar to * and ** ${ }^{*}$ and ${ }^{\# \#}$ indicate $p<0.05$ and $p<0.01$, respectively. Asteriks $\left({ }^{*}\right)$ indicate significant changes compared with Normal. Hashtag $\left({ }^{*}\right)$ indicates significant changes compared with Steatosis.

\section{Human cathepsin D enzyme-linked immunosorbent assay}

Plasma samples were diluted and CatD levels were determined by the CatD enzymelinked immunosorbent assay according to the manufacturers' protocol (Uscn Life Science Inc, Wuhan, China). The detection limit ranges approximately from 46.88 to $3,000 \mathrm{pg} / \mathrm{ml}$. Coefficients of variation (CV\%) for intra- and inter assays are $<10$ and $<12 \%$ respectively. CatD measurements were performed blinded to the histology findings of the study participants.

\section{Statistical analysis}

The data were analysed by performing two-tailed non-paired $t$-tests using GraphPad Prism for comparing the different groups. The data were expressed as mean \pm SEM and considered significant at $p<0.05$. The diagnostic accuracy of the combination ALT with CatD was calculated as described previously. ${ }^{11}$

\section{Results}

\section{General population characteristics of the Cleveland cohort}

ALT was significantly higher in patients with liver inflammation compared to their ageand gender-matched control subjects $(p<0.001)$ (Table 7.1). BMI, low-density lipoprotein (LDL), triglycerides and aspartate aminotransferase (AST) were all 
increased in subjects scored for liver inflammation compared to their matched controls. In line, plasma high-density lipoprotein (HDL) and the AST/ALT ratio were reduced in the group with liver inflammation.

\section{Elevated CatD levels in plasma of patients with liver inflammation}

To determine whether CatD is elevated in NASH patients, CatD and ALT were measured in plasma of the Cleveland cohort. As expected, we found a significant increase of CatD in relation to liver biopsies of patients that demonstrate inflammation, compared to those without liver inflammation (No inflammation vs. inflammation: $p<0.001$ ) (Figure 7.1A). Next, we investigated the correlation between plasma ALT and CatD. Plasma CatD demonstrated a strong positive correlation with ALT (Pearson's $r=0.57, \mathrm{p}<0.001$ ) (Figure 7.1B). These results suggest that plasma CatD levels are linked to the pathogenesis of NASH.

\section{Cleveland cohort}

A

Cathepsin D

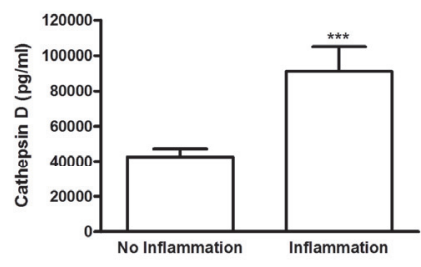

B

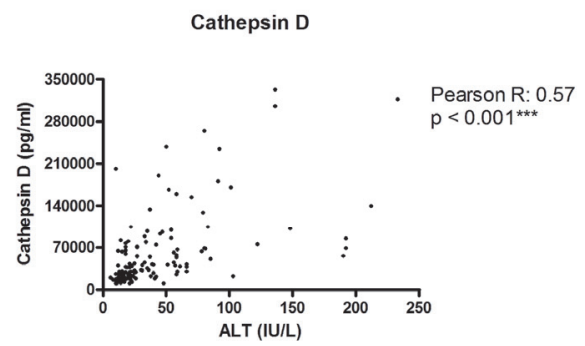

Figure 7.1 CatD associated with ALT and inflammation. (A) CatD in plasma of subjects with or without liver inflammation $(n=127)$. (B) The Pearson correlation test was used to calculate the correlation coefficient $(r)$ between plasma CatD and ALT. $* * *$ indicates $p<0.001$.

\section{Population characteristics of the Maastricht cohort}

To test whether plasma CatD is elevated already during early-stage of NASH, we used the Maastricht cohort consisting of patients with a wide range of fatty liver disease. Clinical characteristics of the patient population from this cohort are presented in Table 7.2. The BMI from the cohort tested, ranged from 30.7 to $73.6 \mathrm{~kg} / \mathrm{m}^{2}$ with an overall average of $46.7 \mathrm{~kg} / \mathrm{m}^{2}$. The average plasma levels of ALT and AST were significantly higher in NASH patients as compared to subjects with a healthy liver $(p=0.018$ and $p=0.019$ respectively). Patients in the NASH group were significantly older compared to patients with a healthy liver $(p=0.021)$ and to those who had steatosis $(p=0.049)$. Importantly, there was no difference with respect to all other parameters (i.e. BMI, plasma total cholesterol, HDL, LDL, triglycerides and the AST/ALT ratio). Liver specimens of $27 \mathrm{NASH}$ patients were histologically classified according to the scoring systems of Brunt and Kleiner. Most NASH subjects (14 out of 27) included 
displayed mild NASH (grade 1), as summarized in Supplementary Table S7.1. The majority of NASH patients showed steatosis score 2, ballooning score 1, lobular inflammation score 1 , and no or little fibrosis.

\section{Plasma CatD distinguishes NASH patients from subjects without hepatic inflammation and correlates with histological classifications of NAFLD}

The level of CatD was significantly higher in subjects with NASH compared to individuals with either simple steatosis or a healthy liver (NASH vs. normal: $p=0.031$; NASH vs. steatosis: $p=0.0065$ ) (Figure 7.2A). More importantly, patients with a mild score for NASH grade, i.e. grade 1, showed an increase in plasma CatD compared to patients without NASH $(\mathrm{p}=0.0018)$ (Figure 7.2B). The same significant trend holds true with respect to lobular inflammation, where CatD levels were already increased with mild disease as observed for NASH grade 1 ( $p=0.0295)$ (Figure 7.2C). In addition, CatD was strongly elevated upon mild steatosis, i.e. score 1 , compared to patients without steatosis $(p=0.019)$, and decreased upon severity of steatosis (Figure 7.2D). Similar to NASH grade, lobular inflammation and steatosis, CatD was primarily elevated upon mild fibrosis (score 1 and 2 ) compared to patients that did not show fibrosis $(p=0.002$ and $p=0.019$, respectively) (Figure 7.2E). Moreover, total cholesterol levels correlated with plasma CatD (Figure 7.2F). In summary, the plasma levels of the lysosomal enzyme CatD are increased in plasma of morbidly obese patients with NASH compared to the patients with a normal liver phenotype or steatosis alone. These data also suggest that plasma CatD correlates specifically with NAFLD severity, already from the early phase, and with cholesterol levels.

\section{In contrast to ALT, CatD correlates with early stages of NASH}

Currently, plasma ALT is the most common diagnostic tool in the clinic to detect hepatocellular injury during NASH. Significantly increased ALT levels were observed in patients with NASH and steatosis (NASH vs. normal: $p=0.018$; steatosis vs. normal: $p=0.019$ ) (Figure 7.3A). While plasma CatD could differentiate NASH from steatosis, there was no difference in ALT concentrations between livers with NASH and steatosis, indicating that CatD correlates better with the spectrum of NAFLD than ALT. Moreover, ALT was only increased upon more advanced degrees of hepatic inflammation (NASH grade 2 vs. grade $0: p=0.003$; NASH grade 3 vs. grade $0: p<0.001$ ) (Figure 7.3B), whereas CatD demonstrated to be elevated already during mild NASH (NASH grade 1). Plasma CatD was significantly higher in the early degrees of steatosis, lobular inflammation and fibrosis, whereas this was not true for ALT (Figure 7.3C-E). A correlation was found between CatD and total cholesterol, whereas no similar correlation could be detected for ALT (Figure 7.3F). Thus, in contrast to ALT, CatD was elevated upon early NAFLD severity and could distinguish NASH from steatosis in adult subjects with morbid obesity. 


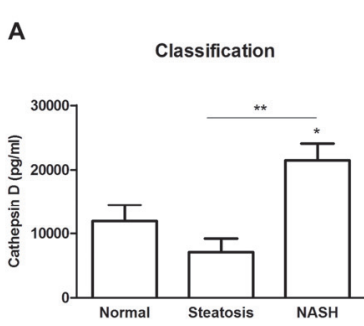

D

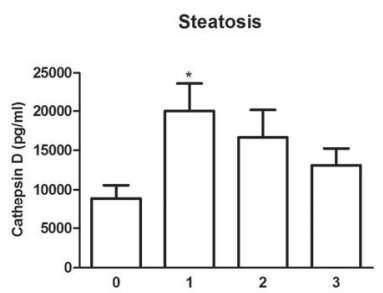

B

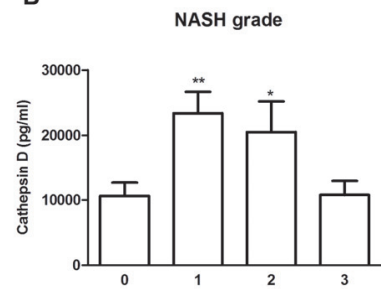

E

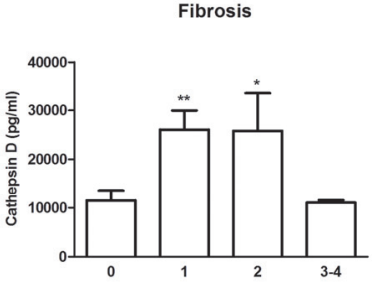

C

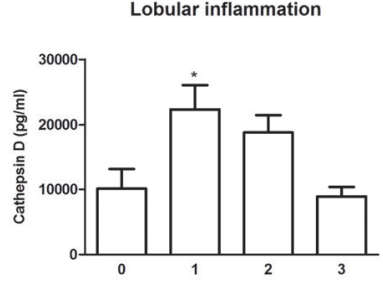

$\mathbf{F}$

Cathepsin D vs. Total cholesterol

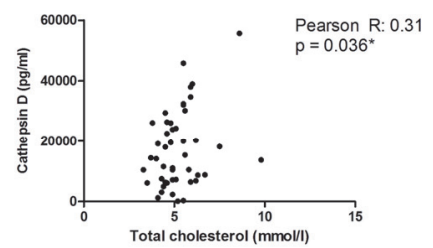

Figure 7.2 CatD in plasma of morbidly obese patients undergoing bariatric surgery. (A) CatD in plasma of severely obese patients with a normal liver phenotype, steatosis and NASH $(n=47)$. According to Brunt's criteria, patients were divided for NASH grade (B). Lobular inflammation (C), steatosis (D) and fibrosis (E) were categorized according to Kleiner's scoring system. (F) The Pearson correlation test was used to calculate the correlation coefficient $(r)$ between plasma CatD and total cholesterol levels. *Significantly different from normal. * and ** indicate $\mathrm{p}<0.05$, and 0.01 , respectively.

\section{Potential clinical significance of using plasma CatD as an additional non-invasive screening method for NASH}

Since our results suggest a strong association between increased plasma CatD and early-stage NASH, we next investigated the potential significance of plasma CatD for the diagnosis of NASH by using the area under the curve (AUC) of plotted receiveroperating characteristic (ROC) curves in the Maastricht cohort. Such a statistical analysis in the Maastricht cohort, with a relative small sample size, gives an indication whether plasma CatD could be potentially clinical relevant for the non-invasive detection of NASH.

\section{Normal \& Steatosis vs. NASH}

First, we compared plasma ALT and CatD levels in normal + steatotic subjects vs. patients with NASH. The AUC of ALT alone was estimated to be $65 \%$, whereas CatD displayed an AUC of $77 \%$ (Figure 7.4A). Notably, combining both enzymes led to an increase in the AUC from $65 \%$ to $77 \%$ compared to ALT alone (Supplemental Figure S7.1). Thus, the addition of CatD to ALT improved the diagnostic value for distinguishing NASH subjects from those who are healthy or have steatosis. 
A

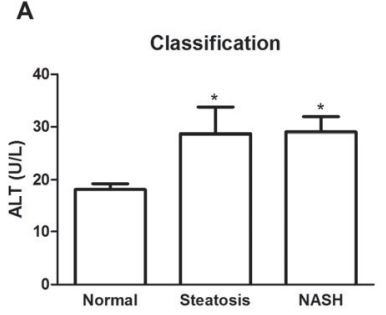

D

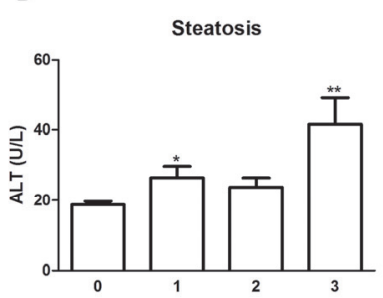

Maastricht cohort

B

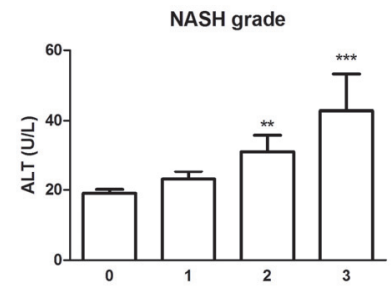

E

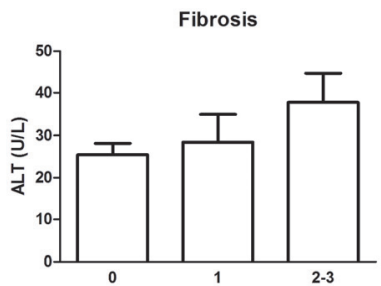

C

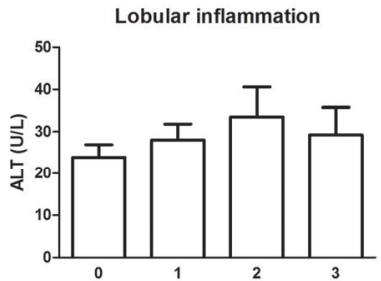

F

ALT vs. Total cholesterol

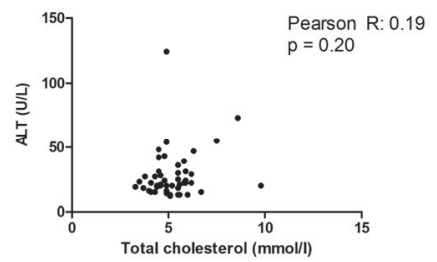

Figure 7.3 Human plasma ALT levels. (A) Plasma ALT in severely obese patients with either a normal, steatotic or NASH liver ( $n=47)$. Patients were classified for NASH grade (Brunt's score) (B) lobular inflammation (C) steatosis (D) and fibrosis (E) (Kleiner's score). (F) The Pearson correlation test was used to calculate the correlation coefficient $(r)$ between plasma ALT and total cholesterol levels *Significantly different from normal. *,** and *** indicate $\mathrm{p}<0.05$, 0.01 , and 0.001 respectively.

Steatosis vs. NASH

Whereas ALT alone demonstrated low predictive power to discriminate steatosis from NASH (AUC: 51\%), CatD demonstrated improved the predictive value with an AUC of 84\% (Figure 7.4B). In comparison to CatD alone, similar diagnostic values were obtained when ALT and CatD were combined with an AUC value of $84 \%$ (Supplemental Figure S7.1). In short, taking plasma CatD into account, the diagnostic prediction for NASH became considerably more accurate than by using ALT alone.

\section{Normal vs. NASH}

Almost similar AUC values were obtained for ALT and CatD (74\% and 73\% respectively) for the differentiation between NASH from healthy individuals (Figure 7.4C). Combining the two enzymes resulted in a minor increase of the AUC value to 77\% (Supplemental Figure S7.1). The relatively high AUC value of ALT alone for the differentiation between NASH and healthy individuals remained roughly equal upon addition of CatD. 
Maastricht cohort

A

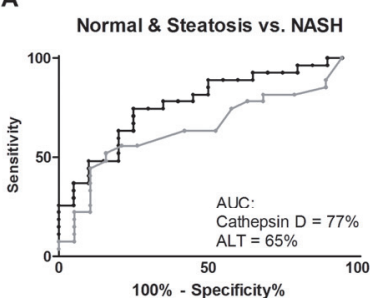

B

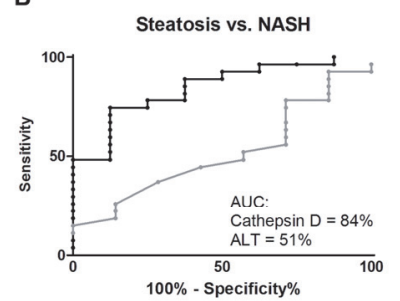

$\rightarrow$ Cathepsin D

$\rightarrow$ ALT

C

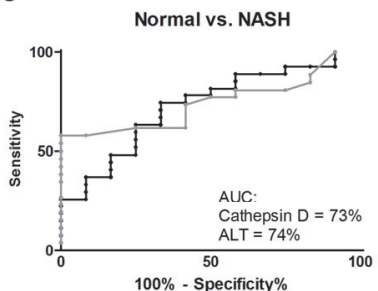

Figure 7.4 Diagnostic accuracy of ALT and CatD for predicting NASH. ROC curve analysis was used to assess the area under the curve (AUC), the sensitivity and specificity of ALT and CatD in predicting NASH for the following comparisons: healthy \& steatotic subjects vs. subjects with NASH (A); subjects with steatosis vs. NASH (B); and normal vs. NASH subjects (C).

To test the intervention effect on plasma CatD levels, we included an additional liver biopsy-proven cohort, which were monitored following a one-year intervention. Before the start of the intervention, CatD levels were increased in adults with NASH compared to adults who did not show signs of liver inflammation (normal vs. NASH: $\mathrm{p}=0.039$ ) (Figure 7.5A), herewith confirming our previous data in this manuscript (Figure 7.1A and Figure 7.2A). Likewise, CatD correlated with total cholesterol levels in plasma (Figure $5 B$ ). The absolute difference of plasma CatD was calculated before and after intervention and demonstrated a significant reduction upon intervention of NASH patients compared to intervention of their healthy individual controls $(p=0.007)$ (Figure $7.5 \mathrm{C}$ ). These data suggest for the first time that plasma CatD can be used in the clinical follow-up of adult NASH patients.

A

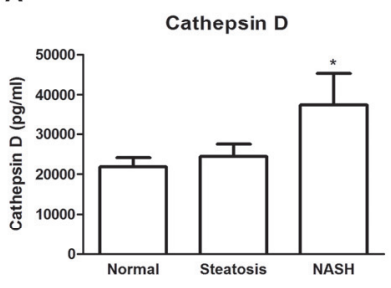

Kuopio cohort

B

Cathepsin D vs. Total cholesterol

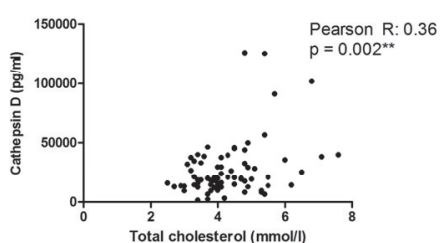

C

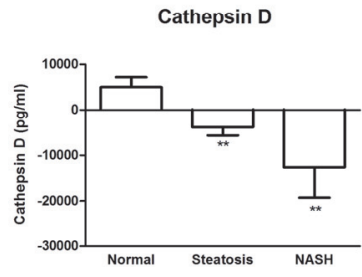

Figure 7.5 Plasma CatD levels in the Kuopio cohort. (A) CatD in plasma of patients with a normal liver phenotype, steatosis and NASH ( $n=74)$. (B) Correlation between CatD and total cholesterol. (C) The absolute difference of plasma CatD was calculated before and after one-year of surgical intervention of normal, steatotic and NASH subjects. 


\section{Discussion}

The exact mechanisms leading to NASH remain largely unknown. Diagnosing NASH is of critical importance in order to prevent further progression into NAFLD-related cirrhosis and end-stage liver disease. Therefore, better understanding of the mechanisms that cause progression to NASH is important.

We have recently established a strong association between CatD in pediatric NASH and children without hepatic inflammation. ${ }^{11}$ In the current paper, we observed an association between plasma CatD and NASH in three biopsy-proven cohorts of adults. Strikingly, whereas we found a decrease of plasma CatD in NASH children, we now have found an increase of CatD in the plasma of adults with NASH. These findings suggest a difference in disease pathology between NASH in adults and in children. Furthermore, our observations point to plasma CatD as a useful non-invasive tool to improve NASH diagnosis in adults.

Under normal conditions, cholesterol is internalized by macrophages and directed to the lysosome for further processing. After hydrolysis, cholesterol is transferred from the lysosome to the cytoplasm where cholesterol is degraded by bile acids or secreted via cholesterol efflux. The difference in pathophysiology between NASH in children and adults could be due to age-dependent changes of the lysosomal compartment. Indeed, lysosomal function has previously been shown to be an age-dependent process, whereby the amount of lysosomes increases upon ageing. ${ }^{25-27}$ These data suggest that at a young age, the amount of lysosomes is low and consequently the cells have difficulties to cope with excessive amounts of cholesterol. As such, upon high-cholesterol circumstances, the stability of the lysosomal membrane is likely to decrease, ${ }^{28}$ which can eventually lead to lysosomal rupture and subsequent lysosomal enzyme release into the cytosol. Lysosomal enzyme secretion into the plasma is hereby prevented and, instead, the cytosolic lysosomal enzymes activate the apoptotic signaling pathway inducing rapid cell death. ${ }^{29}$ This mechanism could explain the reduction of CatD in the plasma of pediatric subjects with NASH compared to subjects without liver inflammation. ${ }^{11}$ In contrast to young cells, older cells possess a higher number of lysosomes, ${ }^{25-27}$ suggesting increased cholesterol storage capabilities inside the cells. It is therefore likely that adult lysosomes can cope better with the cholesterol accumulation and would be less likely to rupture. Nevertheless, cholesterol-filled lysosomes have been shown to induce disturbances in the lysosomal enzyme trafficking pathway ${ }^{9,10}$ leading to increased levels of lysosomal enzymes in plasma. ${ }^{30,31}$ Plasma LDL oxidation can be viewed as a representative parameter of oxidative stress and has shown to be induced in healthy elderly compared to young controls and therefore is strongly associated with the course of ageing. ${ }^{32,33}$ In vitro, particularly the intracellular accumulation of the oxidized LDL (oxLDL) fraction has been shown to enhance extracellular secretion of pro-CatD. ${ }^{34,35}$ Altogether, the observed differences between NASH in children and adults could be explained by the 
fact that secretion of CatD into the plasma is dependent on age-related changes of the lysosomes and is dependent on accumulation of specific lipid species. In line, several studies indeed describe that the histological and pathological characteristics of NASH are age dependent. ${ }^{12-15}$ Further studies are warranted to precisely determine the contribution of lysosomes to NASH disease pathology in children and adults.

With the help of lysosomal enzymes, lysosomes are best known for its primary role in protein degradation. However, protein degradation during NASH seems to be disturbed, as we have recently established a clear association between hepatic inflammation and increased cholesterol accumulation in lysosomes of KCs. ${ }^{5}$ This is underlined by the fact that KCs compromise $15-20 \%$ of the total number of livers cells $^{36}$ and account for the main hepatic uptake of modified cholesterol-rich lipoproteins from the circulation. ${ }^{37}$ Additionally, numerous studies have shown that lysosomal cholesterol accumulation inside macrophages is an event that occurs during inflammation and has been detected in NASH as well as atherosclerosis. ${ }^{5,38}$ These data are in line with previous results showing high cholesterol levels in KCs, as was indicated by numerous cholesterol-filled droplets in KCs of livers of NASH adults, while livers of steatotic patients did not show this typical foam cell-like phenotype. ${ }^{39}$

Lysosomal function is not merely restricted to degradation of proteins, such as cholesterol, but increasing evidence now demonstrates that the lysosomal compartment can also be seen as vesicles that can secrete its content, for example lysosomal enzymes. ${ }^{40,41}$ Cathepsins, a specific class of lysosomal enzymes, have been shown to be significantly involved in mediating the inflammatory response and cholesterol trafficking. ${ }^{42-44}$ Furthermore, we have recently reported that plasma CatD was strongly associated with pediatric NASH. ${ }^{11}$ Likewise, a correlation exists between CatD and atherosclerosis ${ }^{45}$, as well as with a lysosomal storage disease. ${ }^{46}$ Interestingly, both these diseases are predominantly characterized by chronic systemic inflammation and excessive cholesterol storage inside macrophages. In line, in the current paper, we show that CatD is correlated with histological classifications for NASH as well as with cholesterol. These evidences point to a strong link between CatD and cholesterol-mediated inflammation. Therefore, it seems reasonable that lysosomal cholesterol accumulation in KCs, and its subsequent effect on lysosomal enzyme homeostasis, is a central mechanism in the development of NASH.

Several plasma markers that have been tested as a potential screening method for NASH are in relation to apoptosis (cytokeratin-18 fragments) ${ }^{47}$, inflammation (cytokines and adipokines) $^{48}$ and advanced glycation end products. ${ }^{49}$ Numerous studies screening NASH patients for individual cytokeratin-18 (CK-18) fragments described a high diagnostic accuracy with an AUC between 71 and $93 \% .{ }^{50}$ Our observations for analyzing CatD individually to distinguish NASH from steatotic patients are within the same high accuracy range as CK-18 with an AUC of $84 \%$. 
However, whereas CatD correlated with early signs of inflammation, CK-18 represents liver damage and fibrosis. ${ }^{47}$ Combining two biomarkers such as total cytokeratin-18 (M65) with interleukin-6 yielded an AUC of $83 \%$ and $84 \%$ for adiponectin and M65. ${ }^{51}$ In line with this approach, we presented the combination ALT and CatD with a similar high AUC of $84 \%$ for separating NASH from steatotic patients. These data suggest that including one liver-specific parameter (i.e. ALT) could increase the diagnostic accuracy for detecting a liver disease such as NASH. In contrast to ALT, increased CatD levels in the plasma are representative for early-stage hepatic inflammation.

Furthermore, we have shown for the first time that plasma CatD responded to a 1-year follow-up period after a gastric bypass intervention of NASH patients. Although no follow-up liver biopsies were obtained in the current study, gastric bypass intervention has previously been shown to resolve histopathological features of NASH. ${ }^{52}$ While plasma CatD responded to intervention, changes in plasma CK-18 levels following intervention of NASH patients have not been defined so far. ${ }^{53}$ Contradictive results have been found concerning plasma transaminases after surgical intervention, while others demonstrated a correlation between histological improvement of NASH and lower ALT and AST after follow-up ${ }^{54}$, some did not find a difference postoperatively. ${ }^{52}$ Thus, besides NASH diagnosis, plasma CatD can potentially be used in the clinical follow-up and regression of NASH. Future trials are necessary to validate the response of plasma CatD upon intervention.

In the current study, we have found a strong association between plasma CatD and NASH in adults. In contrast to childhood NASH, we now demonstrated elevated CatD levels in plasma of NASH adults. These data suggest the existence of a distinct disease pathology between NASH in children and adults. Plasma CatD holds potential clinical utility as it correlated with histological characteristics of NASH and regression of this disease. Mechanistically, these findings point to the important role of lysosomes in the development of NASH and should be further investigated. 


\section{References}

1. Kopec KL, Burns D. Nonalcoholic fatty liver disease: a review of the spectrum of disease, diagnosis, and therapy. Nutr Clin Pract 2011;26:565-76.

2. Yilmaz Y. Review article: is non-alcoholic fatty liver disease a spectrum, or are steatosis and nonalcoholic steatohepatitis distinct conditions? Aliment Pharmacol Ther 2012;36:815-23.

3. Wieckowska A, McCullough AJ, Feldstein AE. Noninvasive diagnosis and monitoring of nonalcoholic steatohepatitis: present and future. Hepatology 2007;46:582-9.

4. Limdi JK, Hyde GM. Evaluation of abnormal liver function tests. Postgrad Med J 2003;79:307-12.

5. Bieghs V, Hendrikx T, van Gorp PJ, Verheyen F, Guichot YD, Walenbergh SM, Jeurissen ML, et al. The cholesterol derivative 27-hydroxycholesterol reduces steatohepatitis in mice. Gastroenterology 2013;144:167-78 e161.

6. Bieghs V, van Gorp PJ, Walenbergh SM, Gijbels MJ, Verheyen F, Buurman WA, Briles DE, et al. Specific immunization strategies against oxidized low-density lipoprotein: a novel way to reduce nonalcoholic steatohepatitis in mice. Hepatology 2012;56:894-903.

7. Bieghs V, Verheyen F, van Gorp PJ, Hendrikx T, Wouters K, Lutjohann D, Gijbels MJ, et al. Internalization of modified lipids by CD36 and SR-A leads to hepatic inflammation and lysosomal cholesterol storage in Kupffer cells. PLoS One 2012;7:e34378.

8. Ioannou GN, Haigh WG, Thorning D, Savard C. Hepatic cholesterol crystals and crown-like structures distinguish NASH from simple steatosis. J Lipid Res 2013;54:1326-34.

9. Kornfeld S. Trafficking of lysosomal enzymes in normal and disease states. J Clin Invest 1986;77:1-6.

10. Shen D, Wang X, Li X, Zhang X, Yao Z, Dibble S, Dong XP, et al. Lipid storage disorders block lysosomal trafficking by inhibiting a TRP channel and lysosomal calcium release. Nat Commun 2012;3:731.

11. Walenbergh SM, Houben T, Hendrikx T, Jeurissen ML, van Gorp PJ, Vreugdenhil AC, Adriaanse MP, et al. Plasma cathepsin d levels: a novel tool to predict pediatric hepatic inflammation. Am J Gastroenterol 2015;110:462-70.

12. Nobili V. Non-alcoholic fatty liver disease in children and adolescents. Clin Biochem 2014;47:720.

13. Arata M, Nakajima J, Nishimata S, Nagata T, Kawashima H. Nonalcoholic steatohepatitis and insulin resistance in children. World J Diabetes 2014;5:917-23.

14. Giorgio V, Prono F, Graziano F, Nobili V. Pediatric non alcoholic fatty liver disease: old and new concepts on development, progression, metabolic insight and potential treatment targets. BMC Pediatr 2013;13:40.

15. Ovchinsky N, Lavine JE. A critical appraisal of advances in pediatric nonalcoholic Fatty liver disease. Semin Liver Dis 2012;32:317-24.

16. Kalhan SC, Guo L, Edmison J, Dasarathy S, McCullough AJ, Hanson RW, Milburn M. Plasma metabolomic profile in nonalcoholic fatty liver disease. Metabolism 2011;60:404-13.

17. Kasumov T, Edmison JM, Dasarathy S, Bennett C, Lopez R, Kalhan SC. Plasma levels of asymmetric dimethylarginine in patients with biopsy-proven nonalcoholic fatty liver disease. Metabolism 2011; 60:776-81.

18. Kleiner DE, Brunt EM, Van Natta M, Behling C, Contos MJ, Cummings OW, Ferrell LD, et al. Design and validation of a histological scoring system for nonalcoholic fatty liver disease. Hepatology 2005;41:1313-21.

19. Verdam FJ, Dallinga JW, Driessen A, Jonge CD, Moonen EJ, van Berkel JB, Luijk J, et al. Non-alcoholic steatohepatitis: A non-invasive diagnosis by analysis of exhaled breath. J Hepatol 2013;58:543-8.

20. Rensen SS, Slaats Y, Driessen A, Peutz-Kootstra CJ, Nijhuis J, Steffensen R, Greve JW, et al. Activation of the complement system in human nonalcoholic fatty liver disease. Hepatology 2009;50:1809-17.

21. Brunt EM. Pathology of fatty liver disease. Mod Pathol 2007;20 Suppl 1:S40-8.

22. Pihlajamaki J, Gronlund S, Simonen M, Kakela P, Moilanen L, Paakkonen M, Pirinen E, et al. Cholesterol absorption decreases after Roux-en-Y gastric bypass but not after gastric banding. Metabolism 2010;59:866-72.

23. Pihlajamaki J, Kuulasmaa T, Kaminska D, Simonen M, Karja V, Gronlund S, Kakela P, et al. Serum interleukin 1 receptor antagonist as an independent marker of non-alcoholic steatohepatitis in humans. J Hepatol 2012;56:663-70. 
24. Brunt EM, Janney CG, Di Bisceglie AM, Neuschwander-Tetri BA, Bacon BR. Nonalcoholic steatohepatitis: a proposal for grading and staging the histological lesions. Am J Gastroenterol 1999; 94:2467-74.

25. Lynch G, Bi X. Lysosomes and brain aging in mammals. Neurochem Res 2003;28:1725-34.

26. Robbins E, Levine EM, Eagle H. Morphologic changes accompanying senescence of cultured human diploid cells. J Exp Med 1970;131:1211-22.

27. Cho S, Hwang ES. Status of mTOR activity may phenotypically differentiate senescence and quiescence. Mol Cells 2012;33:597-604.

28. Yuan XM, Li W, Olsson AG, Brunk UT. The toxicity to macrophages of oxidized low-density lipoprotein is mediated through lysosomal damage. Atherosclerosis 1997;133:153-61.

29. Singh R, Kaushik S, Wang Y, Xiang Y, Novak I, Komatsu M, Tanaka K, et al. Autophagy regulates lipid metabolism. Nature 2009;458:1131-5.

30. Ungewickell AJ, Majerus PW. Increased levels of plasma lysosomal enzymes in patients with Lowe syndrome. Proc Natl Acad Sci U S A 1999;96:13342-4.

31. Hultberg B, Isaksson A, Sjoblad S, Ockerman PA. Acid hydrolases in serum from patients with lysosomal disorders. Clin Chim Acta 1980;100:33-8.

32. Bottino DA, Lopes FG, de Oliveira FJ, Mecenas Ade S, Clapauch R, Bouskela E. Relationship between biomarkers of inflammation, oxidative stress and endothelial/microcirculatory function in successful aging versus healthy youth: a transversal study. BMC Geriatr 2015;15:41.

33. Gradinaru D, Borsa C, lonescu C, Prada GI. Oxidized LDL and NO synthesis-Biomarkers of endothelial dysfunction and ageing. Mech Ageing Dev 2015.

34. Hoppe G, O'Neil J, Hoff HF, Sears J. Products of lipid peroxidation induce missorting of the principal lysosomal protease in retinal pigment epithelium. Biochim Biophys Acta 2004;1689:33-41.

35. Li W, Yuan XM, Olsson AG, Brunk UT. Uptake of oxidized LDL by macrophages results in partial lysosomal enzyme inactivation and relocation. Arterioscler Thromb Vasc Biol 1998;18:177-84.

36. Kolios G, Valatas V, Kouroumalis E. Role of Kupffer cells in the pathogenesis of liver disease. World J Gastroenterol 2006;12:7413-20.

37. Van Berkel TJ, De Rijke YB, Kruijt JK. Different fate in vivo of oxidatively modified low density lipoprotein and acetylated low density lipoprotein in rats. Recognition by various scavenger receptors on Kupffer and endothelial liver cells. J Biol Chem 1991;266:2282-9.

38. Bieghs V, Rensen PC, Hofker MH, Shiri-Sverdlov R. NASH and atherosclerosis are two aspects of a shared disease: central role for macrophages. Atherosclerosis 2012;220:287-93.

39. Ioannou GN, Haigh WG, Thorning D, Savard C. Hepatic cholesterol crystals and crown-like structures distinguish NASH from simple steatosis. J Lipid Res 2013;54:1326-34.

40. Bordon Y. Immune regulation: lysosomes at the heart of inflammation. Nat Rev Immunol 2011; 11:502.

41. Samie MA, Xu H. Lysosomal Exocytosis and Lipid Storage Disorders. J Lipid Res 2014.

42. Hannaford J, Guo H, Chen X. Involvement of cathepsins B and L in inflammation and cholesterol trafficking protein NPC2 secretion in macrophages. Obesity (Silver Spring) 2013;21:1586-95.

43. Decock J, Obermajer N, Vozelj S, Hendrickx W, Paridaens R, Kos J. Cathepsin B, cathepsin H, cathepsin $\mathrm{X}$ and cystatin $\mathrm{C}$ in sera of patients with early-stage and inflammatory breast cancer. Int J Biol Markers 2008;23:161-8.

44. Sukhova GK, Zhang Y, Pan JH, Wada Y, Yamamoto T, Naito M, Kodama T, et al. Deficiency of cathepsin $S$ reduces atherosclerosis in LDL receptor-deficient mice. J Clin Invest 2003;111:897-906.

45. Moallem SA, Nazemian F, Eliasi S, Alamdaran SA, Shamsara J, Mohammadpour AH. Correlation between cathepsin $D$ serum concentration and carotid intima-media thickness in hemodialysis patients. Int Urol Nephrol 2011;43:841-8.

46. Amritraj A, Peake K, Kodam A, Salio C, Merighi A, Vance JE, Kar S. Increased activity and altered subcellular distribution of lysosomal enzymes determine neuronal vulnerability in Niemann-Pick type C1-deficient mice. Am J Pathol 2009;175:2540-56.

47. Feldstein AE, Wieckowska A, Lopez AR, Liu YC, Zein NN, McCullough AJ. Cytokeratin-18 fragment levels as noninvasive biomarkers for nonalcoholic steatohepatitis: a multicenter validation study. Hepatology 2009;50:1072-8. 
48. Krawczyk K, Szczesniak P, Kumor A, Jasinska A, Omulecka A, Pietruczuk M, Orszulak-Michalak D, et al. Adipohormones as prognostric markers in patients with nonalcoholic steatohepatitis (NASH). J Physiol Pharmacol 2009;60 Suppl 3:71-5.

49. Yilmaz Y, Ulukaya E, Gul OO, Arabul M, Gul CB, Atug O, Oral AY, et al. Decreased plasma levels of soluble receptor for advanced glycation endproducts (SRAGE) in patients with nonalcoholic fatty liver disease. Clin Biochem 2009;42:802-7.

50. Adams LA, Feldstein AE. Non-invasive diagnosis of nonalcoholic fatty liver and nonalcoholic steatohepatitis. J Dig Dis 2011;12:10-6.

51. Grigorescu M, Crisan D, Radu C, Grigorescu MD, Sparchez Z, Serban A. A novel pahtophysiologicalbased panel of biomarkers for the diagnosis of nonalcoholic steatohepatitis. J Physiol Pharmacol 2012;63:347-53.

52. Barker KB, Palekar NA, Bowers SP, Goldberg JE, Pulcini JP, Harrison SA. Non-alcoholic steatohepatitis: effect of Roux-en-Y gastric bypass surgery. Am J Gastroenterol 2006;101:368-73.

53. Dyson JK, McPherson S, Anstee QM. Non-alcoholic fatty liver disease: non-invasive investigation and risk stratification. J Clin Pathol 2013;66:1033-45.

54. Dixon JB, Bhathal PS, Hughes NR, O'Brien PE. Nonalcoholic fatty liver disease: Improvement in liver histological analysis with weight loss. Hepatology 2004;39:1647-54. 


\section{Supplemental table}

Table S7.1 Histological scoring of liver biopsies from NASH subjects of the Maastricht cohort.

\begin{tabular}{llc}
\hline Brunt score & Definition & NASH subjects $(\mathrm{n}=27)$ \\
\hline Grade 1 & Mild & 14 \\
Grade 2 & Moderate & 7 \\
Grade 3 & Severe & 6 \\
Kleiner score & & \\
Steatosis & $<5 \%$ (score 0) & 0 \\
& $5-33 \%$ (score 1) & 7 \\
& $33-66 \%$ (score 2) & 12 \\
Ballooning & $>66 \%$ (score 3) & 8 \\
& None (score 0) & 6 \\
& Few balloon cells (score 1) & 18 \\
Lobular inflammation & Prominent ballooning (score 2) & 3 \\
& None (score 0) & 2 \\
& $<2$ foci per 200x field (score 1) & 14 \\
& 2-4 foci per 200x field (score 2) & 7 \\
Fibrosis & $>4$ foci per 200x field (score 3) & 4 \\
& None (score 0) / Nondefined & $10 / 2$ \\
& Perisinusoidal or periportal (score 1) & 6 \\
& Perisinusoidal and portal/periportal (score 2) & 5 \\
& Bridging fibrosis (score 3) & 2 \\
& Extensive bridging fibrosis, cirrhosis (score 4) & 2 \\
\hline
\end{tabular}

\section{Supplemental figure}

Figure S7.1 Diagnostic accuracy of the combination ALT and CatD for predicting NASH.

Maastricht cohort

\section{ALT + Cathepsin D}
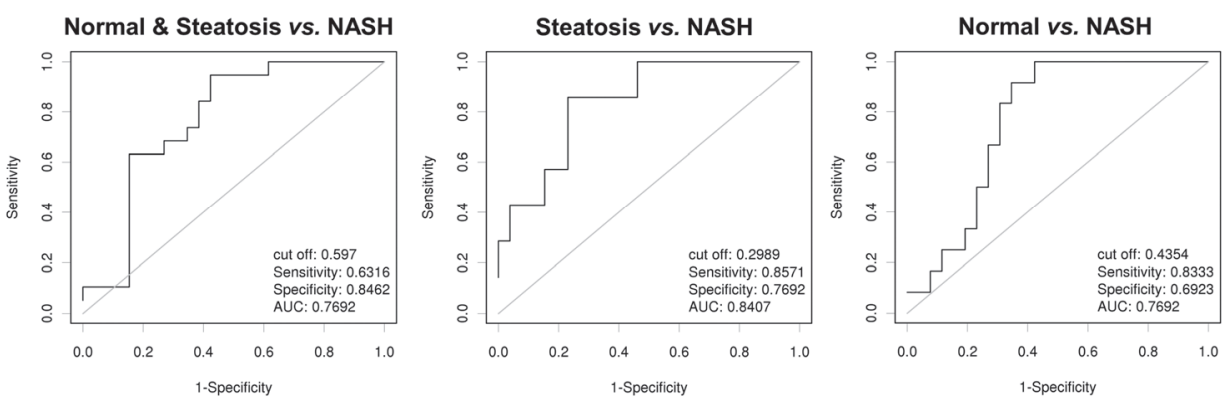


\section{Chapter 8}

Lysosomal cholesterol accumulation: driver on the road to inflammation during atherosclerosis and non-alcoholic steatohepatitis

T Hendrikx, SMA Walenbergh, MH Hofker, R Shiri-Sverdlov

Obes Rev. 2014;15:424-433 


\section{Abstract}

Many studies show an association between the accumulation of cholesterol inside lysosomes and the progression towards inflammatory disease states that are closely related to obesity. While in the past the knowledge regarding lysosomal cholesterol accumulation was limited to its association with plaque severity during atherosclerosis, recently a growing body of evidence indicates a causal link between lysosomal cholesterol accumulation and inflammation. These findings make lysosomal cholesterol accumulation an important target for intervention in metabolic diseases that are characterized by the presence of an inflammatory response.

In this review, we aim to show the importance of cholesterol trapping inside lysosomes to the development of inflammation by focusing on cardiovascular disease and non-alcoholic steatohepatitis (NASH) in particular. We summarize current data supporting the hypothesis that lysosomal cholesterol accumulation is playing a key role in the development of inflammation during atherosclerosis and NASH. In addition, potential mechanisms by which disturbed lysosomal function can trigger the inflammatory response, the challenges in improving cholesterol trafficking in macrophages and recent successful research directions will be discussed. 


\section{Introduction}

Nowadays, obesity is seen as one of the leading health concerns worldwide. The dramatically increasing occurrence of obesity in Western countries is accompanied by an increase in the prevalence of related conditions such as cardiovascular disease, stroke, type 2 diabetes, fatty liver disease and certain types of cancer. Also among children, obesity is causing a broad range of health problems including high blood pressure and elevated blood cholesterol levels. Therefore, there is a critical need to improve treatment options for obesity-related inflammatory disorders. In order to do so, the etiology of the diseases needs to be clarified. A common feature of obesity and related conditions is the presence of inflammation in different tissues, such as the vessel wall during atherosclerosis and the liver during non-alcoholic steatohepatitis (NASH). Although the exact mechanism that triggers and sustains this inflammatory state is not known, macrophages have been shown to play a central role. Interestingly, recent investigations have indicated lysosomal cholesterol accumulation in macrophages as a novel pathway that contributes to the inflammatory response during cardiovascular disease and $\mathrm{NASH}^{1-3}$ In this manuscript, we will discuss the role of cholesterol trapping inside lysosomes of macrophages during atherosclerosis and $\mathrm{NASH}$ and its contribution to the sustained inflammatory response. Additionally, we will describe potential targets for improving cholesterol trafficking in macrophages and recent successful research directions.

\section{Foam cell formation}

The central role of macrophages in triggering inflammation in different tissues has been extensively reviewed by others. ${ }^{4,5}$ Upon activation by different stimuli, tissue resident macrophages secrete pro-inflammatory cytokines, resulting in the recruitment and activation of macrophages from the circulation. During atherosclerosis and NASH, which are characterized by the presence of inflammation, the accumulation of lipid-laden macrophages, termed foam cells, is critical for, and increases with the progression of disease. ${ }^{6,7}$ These so-called lipid-laden macrophages are formed upon uptake of lipoproteins containing triglycerides (TG) and cholesterol. Lipoproteins rich in TG can induce TG accumulation leading to foam cell formation via receptor-mediated uptake of triglyceride-rich lipoproteins (TGRLs) and free fatty acids. These free fatty acids are generated from triglyceride-rich chylomicrons and very low-density lipoproteins (VLDL), which are metabolized in the cells or stored as TG. $^{8}$

Besides TGRLs, cholesterol-rich low-density lipoproteins (LDL) are also known to be involved in foam cell formation. Importantly, a clear separation must be addressed between the uptake of non-modified LDL and the uptake of LDL particles that are modified. Non-modified LDL is taken up by cells via receptor-mediated endocytosis, a 
mechanism by which cells recognize extracellular ligands by the LDL receptor (LDLR) and subsequently internalize these ligands by inward budding of the plasma membrane. ${ }^{9}$ Upon binding to the LDLR, LDL is internalized as clathrin coated vesicles that are then converted into endosomes. Here, a more acidic $\mathrm{pH}$ (4.5 to 5) exists, which leads to the dissociation of the ligand-receptor complex. The LDLR is recycled to the surface of the cell, and the endosomes combine with lysosomes which contain acid hydrolases that can easily degrade all components of LDL. After hydrolysis, cholesterol is transferred into the cytoplasm via Niemann-Pick type C (NPC) proteins where it can be further degraded to bile acids or secreted via cholesterol efflux transporters. The cholesterol derived from LDL in the lysosome was found to be responsible for the regulation of processes that stabilize cholesterol content of the cell. $^{9}$ First of all, it suppresses the activity of 3-hydroxy-3methylglutaryl coenzyme A reductase (HMG-CoA reductase), which catalyzes a rate-limiting step in cholesterol production. ${ }^{10}$ Next to that, LDL-derived cholesterol is able to activate a cholesterolesterifying enzyme, acyl CoA: cholesterol acyl-transferase (ACAT), thereby storing excess cholesterol as cholesteryl droplets in the cytoplasm. ${ }^{11}$ Additionally, the LDL receptor is subject of feedback regulation as LDL suppresses the transcription of the LDL receptor gene, thereby regulating the number of LDL receptors on the cell surface. ${ }^{10}$ Taken together, several mechanisms exist to prevent the formation of foam cells from non-modified LDL uptake.

Unlike the uptake of non-modified LDL, in vitro studies have shown that the uptake of oxidatively modified LDL by macrophages can contribute to cholesterol accumulation and subsequent formation of foam cells. ${ }^{12,13}$ These oxidized LDL fractions are taken up by scavenger receptors (SRs), present on macrophages, which were proven not to be downregulated in response to an increase in cellular cholesterol content, indicating that SR-mediated LDL uptake is the main cause for foam cell formation. ${ }^{14}$ The two main SRs responsible for the uptake of modified LDL by macrophages are scavenger receptor A (SR-A) and CD36. ${ }^{14}$ Next to SR-mediated uptake of modified LDL, but to a much lesser extent, the phagocytic uptake of aggregated LDL by macrophages is an additional proposed mechanism that contributes to foam cell formation. ${ }^{15}$ In summary, whereas the processes involved in LDL uptake are tightly regulated and protect cells from becoming foam cells, the uptake of modified LDL was found to be responsible for the development of foamy macrophages.

During obesity, apart from increased levels of cholesterol-rich LDL, also increased oxidative stress is present, leading to the increased conversion of LDL to oxLDL. ${ }^{16,17}$ Under healthy conditions, the fraction of oxLDL in the plasma is very small compared to LDL $(0.001 \%){ }^{18,19}$ In patients with the metabolic syndrome, the fraction of oxLDL was shown to be increased to $0.6-1.8 \%$ compared to controls ${ }^{20}$, whereas patients with acute coronary heart disease had fractions up to $5 \% .{ }^{21}$ While the uptake of cholesterol-rich LDL is tightly regulated and inhibited upon excess amounts, uptake of oxLDL is not inhibited. ${ }^{22}$ As such, it is important to keep the fraction of oxLDL as low as possible. 


\section{Disturbed intracellular cholesterol trafficking in macrophages during inflammation}

Circulating modified cholesterol-rich LDL is taken up by macrophages and initially directed to lysosomes for degradation. Lysosomes are ubiquitous membrane-bound organelles that contain acid hydrolases, i.e. digestive enzymes that are responsible for the correct degradation of the lysosomal content. Under normal conditions, cholesterol becomes hydrolyzed and the free cholesterol that is formed can be transferred by the Niemann-Pick type C (NPC) proteins 1 and 2 from the lysosome to the cytoplasm. The majority of cholesterol is then used for bile acid formation or mobilized to the plasma membrane, whereas excess cholesterol is either stored as cholesteryl esters (CE) in cytoplasmic lipid droplets or excreted during reverse cholesterol transport via the ATP-binding cassette transporters (ABCs) A1, G1, B4, or via the scavenger receptor class $B$ type I (SR-BI). By this means, the cells are provided with sufficient cholesterol for cellular functions, without overloading. ${ }^{23}$

While previously it was believed that foamy macrophages are responsible for the observed inflammatory response, recent studies suggest that the intracellular cholesterol distribution plays an important role in triggering inflammation. Specifically, cholesterol accumulation inside lysosomes is found to be associated with an inflammatory state. ${ }^{1,24}$ An important lysosomal enzyme that hydrolyzes CE and TG from internalized lipoproteins is lysosomal acid lipase (LAL). In humans, LAL is encoded by the LIPA gene and mutations of this gene lead to LAL deficiency and subsequent lysosomal cholesterol accumulation, which involves premature cardiovascular disease. ${ }^{25}$ Moreover, mice with a deficiency in LAL are, analogous to patients with LAL deficiency, characterized by lysosomal cholesterol accumulation and inflammation. ${ }^{26}$ Likewise, exogenous LAL administration in LDL receptor knockout $\left(\mathrm{Ldll}^{-/}\right)$mice on a high-fat, high-cholesterol (HFC) diet resulted in decreased lysosomal cholesterol storage and to a significant reduction in hepatic inflammation and atherosclerotic lesion size. ${ }^{27}$

While non-modified LDL is degraded normally and stored mainly in extra-lysosomal compartments, cholesterol delivered to macrophages in the form of oxLDL, which is increasingly present during obesity, fails to efflux normally and was found to result in lysosomal cholesterol accumulation. ${ }^{13}$ In an in vitro set-up, the uptake of oxLDL by macrophages was shown to partially inactivate lysosomal enzymes, thereby leading to increased cholesterol storage inside lysosomes. ${ }^{28}$ It was recently shown that the uptake of oxLDL, and not native LDL, by macrophages is a trigger for inflammation. ${ }^{2,3,12,29-31}$ Thus, unlike the uptake of native LDL, oxLDL uptake by macrophages results in the accumulation of cholesterol in lysosomes, thereby triggering the inflammatory response.

Lysosomal cholesterol accumulation is observed in two important aspects related to obesity, being atherosclerosis and NASH. In foam cells of advanced atherosclerotic plaques, most of the cholesterol appears to be trapped in lysosomes. Electron 
microscopic observations of lesions confirmed that much of the accumulated lipids in foam cells occurred within large, lipid-engorged lysosomes. ${ }^{32}$ These findings were confirmed in both human lesions and atherosclerotic lesions in animal models of the disease. ${ }^{33-35}$ More recently, our group has shown that also in the liver the uptake of oxLDL by Kupffer cells, the resident macrophages of the liver, is correlated with lysosomal cholesterol accumulation and increased hepatic inflammation during NASH development in $\mathrm{Ldlr}^{-/}$mice. ${ }^{29,36}$ Taken together, these studies suggest that the intracellular cholesterol distribution plays a critical role in the onset of inflammation and in the pathogenesis of conditions related to obesity, such as atherosclerosis and NASH.

\section{Mechanisms linking lysosomal cholesterol accumulation and inflammation}

Currently, several mechanisms can link lysosomal cholesterol accumulation and inflammation. In this review, we focus on the effect of cholesterol trapping in lysosomes that concerns autophagy, inflammasome activation and lysosomal cathepsin release (Figure 8.1).

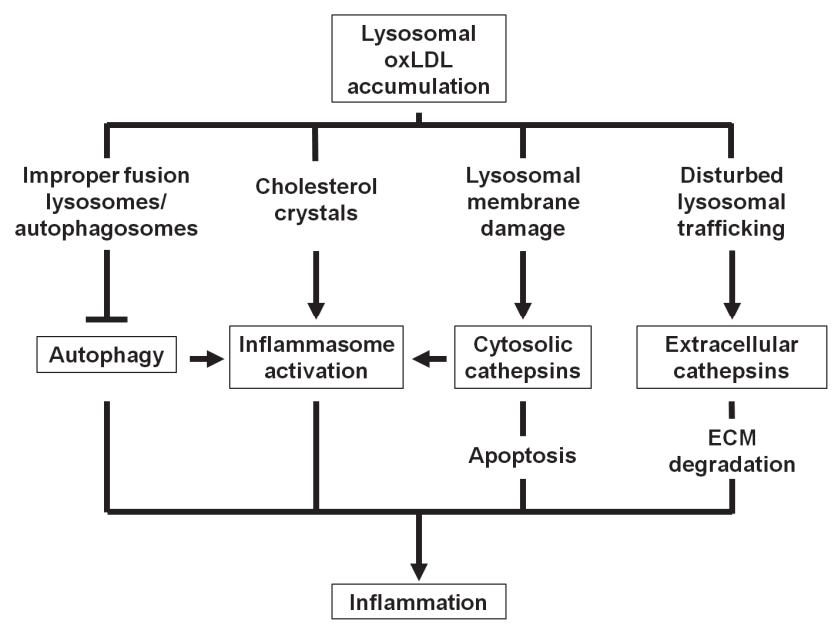

Figure 8.1 Potential mechanisms linking lysosomal oxLDL accumulation and inflammation. Lysosomal oxLDL accumulation can lead to inflammation via several mechanisms. First, lysosomal cholesterol trapping can lead to inhibition of autophagy due to improper fusion between lysosomes and autophagosomes. Next, as a result of lysosomal cholesterol accumulation, cholesterol crystals will be formed and subsequent inflammasome activation will occur. Due to lysosomal membrane damage, cathepsins may be released into the cytosol leading to mitochondrial dysfunction and apoptosis. Alternatively, cathepsins may be secreted from the cell due to disturbed lysosomal trafficking, thereby degrading the extracellular matrix (ECM). Finally, apart from cholesterol crystals, cytosolic cathepsins and reduced autophagy can activate inflammasomes, thereby potentially leading to increased inflammation. 


\section{Autophagy}

Autophagy is an important process for the degradation of cellular components such as misfolded proteins or damaged cell organelles. The accumulation of cholesterol inside lysosomes can result in lysosomal dysfunction and inhibition of autophagy, as a block in autophagy is observed during different lysosomal storage disorders (reviewed elsewhere). ${ }^{37-40}$ Lysosomes play a fundamental role during autophagy as these organelles fuse with autophagosomes to digest their content. This process can produce energy under starvation but is also involved in maintenance of homeostasis through the degradation and resynthesis of damaged components. Recent studies have discovered new protective functions for autophagy, such as the regulation of intracellular lipid stores ${ }^{41}$, as well as a role in immunity, cell death and inflammation. ${ }^{37}$ A possible explanation for disturbed autophagy is that lipid accumulation alters membrane structure, thereby leading to improper fusion between lysosomes and autophagosomes. Changes in membrane lipid composition significantly reduced the number of fusion events between lysosomes and autophagosomes, both in vitro as well as in vivo. ${ }^{42}$ In addition, it was shown that cholesterol abnormalities in lysosomal storage disorders affect soluble $\mathrm{N}$-ethylmaleimide attachment protein receptors (SNAREs), which are key components of the fusion machinery between lysosomes and autophagosomes. ${ }^{43}$

Besides evidence of disturbed autophagy in lysosomal storage disorders, it may also play a prominent role in obesity and related complications. During obesity, adipose tissue expansion occurs due to increased size and number of adipocytes. Interestingly, autophagy was found to regulate adipose mass and differentiation as decreased autophagy in adipocytes was reported to result in conversion of white adipocytes to brown adipocytes. ${ }^{44}$ Autophagy deficiency in adipose tissue was found to be protective against HFC-induced obesity in mice and resulted in increased amount of brown adipose tissue, thereby stimulating weight loss and improving insulin sensitivity. ${ }^{44}$ In line with these studies, recent data show that autophagy in adipocytes of obese individuals is enhanced. ${ }^{45}$

Several other studies exploring the role of autophagy indicate that macrophage autophagy is protective against inflammation. ${ }^{46-48}$ Recently, different groups shed light on the role of autophagy during atherosclerosis. Increased apoptosis and oxidative stress in lesional macrophages, more plaque necrosis and worsened lesional efferocytosis was found in hyperlipidemic mice deficient for autophagy after an highfat diet, indicating a protective role of autophagy in advanced atherosclerosis. ${ }^{46}$ Additionally, Razani et al showed that autophagy becomes dysfunctional upon plaque progression in $\mathrm{ApoE}^{-/-}$mice during atherogenesis. These data indicate a protective role for macrophage autophagy during atherogenesis.

As for the liver, dysfunctional autophagy due to lysosomal cholesterol accumulation may play an important role during the development of NAFLD and NASH. Recently, the involvement of autophagy during hepatocyte lipid metabolism was 
demonstrated. $^{41}$ Lipid droplets and autophagic components were shown to be associated during nutrient deprivation and inhibition of autophagy in cultured hepatocytes and mouse liver increased triglyceride storage in lipid droplets, indicating an essential function for autophagy in hepatic lipid stores. ${ }^{41}$ Besides the regulation of lipid content by autophagy, it was found that autophagic activity is highly dependent on nutritional status. Whereas autophagy levels are elevated upon starvation and short-term exposure to high lipid levels, chronic lipid exposure was found to be correlated with impaired autophagy. Both in genetic (ob/ob) and dietary (high-fat induced) mice models having long-term lipid availability resulted in inhibition of hepatic autophagy. ${ }^{49}$ Mice upon an HFC diet were found to have impaired hepatic autophagic function as demonstrated by a decreased mobilization of lipids into the autophagic compartment. Thus, besides that dysfunctional autophagy may promote hepatic steatosis, excessive lipid accumulation in hepatocytes may be a mechanism leading to decreased autophagic function. The ability of excessive lysosomal lipid accumulation to impair autophagy provides another mechanism for the progression of simple steatosis to NASH and its complications.

Taken together, these data indicate that dysfunctional autophagy due to lysosomal cholesterol accumulation is involved in triggering inflammation in different organs during obesity-related disorders.

\section{Inflammasome activation}

Other evidence suggest a link between lysosomal cholesterol accumulation, lysosomal damage and the activation of the inflammasome. ${ }^{50-52}$ Inflammasomes are multiprotein complexes that control the activation of caspase-1, which is constitutively expressed in the cytosol in the inactive pro-form. Upon activation by different stimuli, caspase- 1 triggers the maturation and release of the pro-inflammatory cytokines interleukin-1 $\beta$ and interleukin-18 to engage in the innate immune defense (i.e. promoting lymphocyte activation, leukocyte infiltration, and NF-kB activation). ${ }^{52}$ Upon the different known stimuli for inflammasome activation are signals such as cholesterol crystals, metabolic stress and the production of reactive oxygen species (ROS). ${ }^{52,53}$ In macrophages incubated with CE, the accumulation of free cholesterol in lysosomes after phagocytic uptake was found to result in the formation of cholesterol crystals. ${ }^{54}$ Electron microscopy and acid phosphatase cytochemistry of lysosomes in cholesteryl ester-loaded cells confirmed that cholesterol crystal formation occurred within lipid-loaded lysosomes. ${ }^{54}$ On top, cholesterol crystallization was found in association with inflammatory cells. ${ }^{55}$ These data suggest that lysosomal cholesterol accumulation in macrophages is associated with cholesterol crystal formation and may lead to inflammation via subsequent inflammasome activation.

Also during cardiovascular disease cholesterol crystallization and inflammasome activation was observed. In atherosclerotic lesions, cholesterol crystals are formed in 
macrophages and were shown to induce phagolysosomal membrane rupture, leading to the release of the phagolysosomal content into the cytosol. ${ }^{51}$ In addition, phagolysosomal damage caused by cholesterol crystals, both in vitro and in vivo, activates the NLRP3 inflammasome, indicating a potential mechanism of inflammation in the vascular wall. ${ }^{51,56}$ The involvement of inflammasome activation in atherosclerosis has been further described in numerous studies. ${ }^{47,51,57,58}$ Although in one study no difference was found between the inflammasome deficient and wildtype controls in the field of atherosclerosis, ${ }^{59}$ other studies using specific knockout models for different parts of the inflammasome (NLRP3, Caspase-1, ASC) showed that inflammasome activation is involved in sustaining the inflammatory response during atherosclerosis. ${ }^{47,51,57,58}$ Bone marrow transplantation with NLRP3-deficient and ASC (also known as PYCARD)-deficient bone marrow into $\mathrm{Ldll}^{-/-}$mice resulted in markedly decreased early atherosclerosis after receiving a high-cholesterol diet, indicating that macrophage-specific inflammasome activation plays an important role during atherosclerosis. ${ }^{51}$ In line with these observations from in vivo studies, it was recently found that in aortas of patients with coronary atherosclerosis, NLRP3 was overexpressed. Moreover, the aortic NLRP3 expression is correlated with the severity of coronary artery disease and the atherosclerotic risk factors, further indicating the importance of inflammasome activation due to lysosomal cholesterol accumulation during cardiovascular disease. ${ }^{60}$

Cholesterol crystals were also found in the livers of $\mathrm{Ldll}^{-/-}$mice upon HFC feeding. ${ }^{1}$ These findings suggest that also during $\mathrm{NASH}$, inflammasome activation may play an important role in triggering the observed inflammatory response. Previously, using Caspase $-1^{-1-}$ mice the role of inflammasomes during the development of NASH was investigated. ${ }^{61,62}$ Caspase-1 deficiency resulted in protection from diet-induced hepatic steatosis, inflammation and early fibrogenesis. Furthermore, $1 L 16^{-/-}$mice had less steatohepatitis and fibrosis compared to controls upon HFC feeding, indicating the involvement of inflammasome-mediated IL1 $\beta$ signaling. Interestingly, also in patients suffering from NASH, cholesterol crystals were recently found to be present in the liver, whereas in patients with simple steatosis this was not the case. ${ }^{63}$ This further indicates the association between cholesterol crystal formation, potentially due to lysosomal cholesterol accumulation, and inflammation, suggesting that inflammasome activation may also be involved in human NASH.

\section{Lysosomal cathepsin release}

Cathepsins, named after the Greek kathepsein (to digest), were discovered in the second half of the $20^{\text {th }}$ century as part of the lysosomal cysteine proteases. Initially, they were shown to localize in lysosomes and endosomes and served to degrade internalized content and to be involved in the clearance of misfolded proteins. Further, cathepsins are known to be optimally active in a slightly acidic environment 
and are mostly unstable at neutral $\mathrm{pH}$. However, recent discoveries have found that cathepsins can be secreted and exert different functions in the extracellular spaces. Importantly, cathepsins were shown to play an important role in apoptosis, antigen presentation in the immune system, collagen turnover in bone and cartilage and hormone processing (reviewed elsewhere) ${ }^{64}$ and to be involved in several inflammatory diseases including obesity ${ }^{65,66}$ and atherosclerosis. ${ }^{67}$

Relevantly, lysosomal cholesterol accumulation may induce leakage of cathepsins out of the lysosomes. First, cathepsins may be released into the cytosol due to increased lysosomal membrane permeability. Indeed, oxLDL uptake in human macrophages was found to immediately damage the lysosomal membrane, leading to the release and relocation of acidic lysosomal enzymes as indicated by increased cytosolic activity of cathepsin $L$ and relocation of cathepsin $D$ to the cytoplasm. ${ }^{28}$ Cytosolic cathepsins were found to be involved in apoptosis and, in contrast to caspases, are already active when released from the lysosome. ${ }^{68-70}$ Therefore, relocation of cathepsins into the cytosol, where they may act as cleavage enzymes for apoptosis, may contribute to the development of the necrotic core and therefore may be considered an atherosclerosis-stimulating factor. ${ }^{71}$ Next to that, cytosolic cathepsins are able to activate inflammasomes, thereby potentially leading to inflammation. ${ }^{56}$

In addition, lysosomal cholesterol accumulation can lead to inhibition of trafficking of lysosomes along the plasma membrane, thereby potentially increasing the secretion of lysosomal enzymes into the circulation. ${ }^{72}$ Extracellularly, cathepsins were shown to play an import role in remodeling of the extracellular matrix (ECM). ${ }^{73}$ The ECM consists of elastins, collagens and proteoglycans, which all are known as substrates for cathepsins. $^{73}$ Relevantly, it was shown that inflammatory cytokines augment cathepsin expression and activity resulting in extracellular matrix proteolysis. ${ }^{67,74}$ By degrading the ECM, cathepsins may be involved in different pathologies as this may facilitate the migration and invasion of macrophages. Taken together, these data indicate that lysosomal cholesterol trapping can lead to inflammation via intracellular or extracellular release of cathepsins.

Many studies have described that cathepsins play a role during atherosclerosis (reviewed elsewhere). ${ }^{75}$ While normal arteries express little or no cathepsins, in atherosclerotic vessels these proteins are abundantly expressed and secreted. More specific, it was shown that cathepsin $\mathrm{S}$ and $\mathrm{K}$ mRNA levels and proteins were much more present in atherosclerotic lesions than in normal arteries. ${ }^{67} \mathrm{~A}$ similar increase in the expression of lysosomal cathepsins $S, L$ and $B$ was found in atherosclerotic lesions of ApoE-deficient mice. Cathepsin protein expression was localized in macrophages and/or in lipid-rich areas. ${ }^{76}$ Additionally, cathepsins show differential expression in various stages of atherosclerosis, and in vivo studies using knockout mice revealed that deficiency of cathepsin S, K or L reduces diet-induced atherosclerosis. ${ }^{77-79}$ Also in human atherosclerotic lesions it has been reported that several cathepsins, including cathepsins $B, D, L$, and S, are expressed in a lesion-dependent manner. ${ }^{80}$ These data 
further indicate that lysosomal cathepsins are involved in atherosclerosis development in humans.

Cathepsin release was also found to play an import role during the development of liver diseases. In a dietary murine model of NAFLD, inactivation of cathepsin B protects against the development of hepatic steatosis ${ }^{81}$ and cathepsin B knockout animals were shown to be protected against hepatic injury. ${ }^{82}$ Furthermore, cathepsin $B$ was found to be implicated in progression of liver fibrosis ${ }^{83}$ and the release of cathepsin B into the cytoplasm is also observed in human liver specimens with NAFLD and correlates with disease severity. ${ }^{81}$ Similarly, we have recently shown that lysosomal cholesterol storage in Kupffer cells of $\mathrm{Ldll}^{-/}$mice is correlated with increased activity of cathepsin D in the liver. ${ }^{1}$ Mechanistically, cathepsin B release into the cytosol may contribute to the progression from NAFLD to NASH by leading to mitochondrial dysfunction and apoptosis. ${ }^{81,82}$ In vitro, it was found that mitochondrial dysfunction occurred several hours after lysosomal permeabilization and cathepsin B redistribution into the cytoplasm. Relevantly, either pharmacological or genetic inhibition of cathepsin B preserved mitochondrial function. Moreover, cathepsin B inactivation protected mitochondria, decreased oxidative stress, and attenuated hepatic injury in vivo. ${ }^{82}$ Taken together, these data indicate that the release of lysosomal cathepsins into the cytoplasm may induce mitochondrial dysfunction, thereby leading to cell death and trigger inflammation. In conclusion, improper functioning of cathepsins may play an important role in conditions involving liver injury such as NASH.

\section{Lysosomal cholesterol accumulation as potential target for therapy}

As different mechanisms were described for the involvement of cholesterol trapping inside lysosomes of macrophages in triggering inflammation, the use of lysosomal cholesterol accumulation as a potential target for novel therapy options in described disorders is highlighted.

A possible way to elucidate the effect of lysosomal cholesterol accumulation is stimulating the transport of cholesterol from lysosomes into the cytoplasm. However, this is a challenging issue as it was previously shown that lysosomal cholesterol derived from oxLDL is resistant to efflux. ${ }^{84-86}$ Moreover, it was shown that, although acLDL-derived CE is usually efficiently hydrolyzed and cleared, a 3-day pre-incubation of macrophages with oxLDL impaired the subsequent ability of lysosomes to hydrolyze acLDL-derived $\mathrm{CE}^{3}{ }^{3}$ Besides, studies in both arteries and cells in culture suggest that the cholesterol in lysosomes is trapped and cannot be decreased simply by inhibiting further uptake of lipoproteins or by increasing efflux of extra-lysosomal cholesterol stores. ${ }^{84,87,88}$ Thus, to therapeutically target lysosomal cholesterol accumulation, unique new methods must be investigated. 
The intracellular trafficking of cholesterol from the lysosome to the cytoplasm is mainly regulated by the proteins NPC1 and NPC2. Whereas NPC1 is a protein that resides in the membrane of endosomes and lysosomes, NPC2 is present inside lysosomes and binds cholesterol. ${ }^{89}$ A mutation in either of the genes for NPC1 $195 \%$ of cases) and NPC2 (5\% of cases) results in Niemann-Pick disease type C, which is an autosomal recessive, lysosomal lipid storage disease. The organs of humans and mice carrying the $\mathrm{NPC1}^{-/}$genotype are characterized by two kinds of pathological alterations. First, there is an elevation of the concentration of unesterified cholesterol in the lysosomes of all cells in the body. Second, this mutation results in inflammation in many tissues like the spleen, liver, and lung. ${ }^{90}$ In $\mathrm{Npc}^{-1-}$ mice, the administration of 2-hydroxypropyl- $\beta$-cyclodextrin was shown to overcome the lysosomal transport defect and led cholesterol flow to the cytosolic compartment, thereby reducing lysosomal cholesterol accumulation. ${ }^{90}$ This reduction in lysosomal cholesterol content was associated with reduced sterol synthesis and expression of macrophageassociated inflammatory genes. ${ }^{90}$ These findings were confirmed by others where treatment of NPC1 or NPC2 mutant cells with 2-hydroxypropyl- $\beta$-cyclodextrin resulted in the release of cholesterol stored in lysosomes. ${ }^{91,92}$ Another promising treatment option is the neurosteroid allopregnanolone, as a single dose at postnatal day 7 almost doubled the lifespan of $\mathrm{Npc1}^{-/}$mice. ${ }^{93}$ Allopregnanolone was shown to reduce cholesterol accumulation, improve autophagic/lysosomal function and reduce inflammation $^{94}$, further supporting the use of this drug in diseases characterized by lysosomal cholesterol accumulation such as atherosclerosis and NASH. So far, the use of allopregnanolone in the treatment of atherosclerosis or NASH has not been investigated. Therefore, it is highly recommended that future studies will determine the effect of allopreganolone in described diseases.

Other mediators of intracellular cholesterol transport are liver $\mathrm{X}$ receptor (LXR) activating oxysterols such as 27 -hydroxycholesterol (27HC). $27 \mathrm{HC}$ is an intermediate in bile acid synthesis and the major oxysterol present in the human circulation. In line with greatly reduced $27 \mathrm{HC}$ production in NPC1-deficient cells, incubation with $27 \mathrm{HC}$ was shown to dramatically reduce lysosomal cholesterol in $\mathrm{NpC1}^{-1-}$ fibroblasts. ${ }^{95}$ Similarly, injecting $\mathrm{Ldlr}^{-/}$mice with $27 \mathrm{HC}$ during or after an HFC diet resulted in reduced lysosomal cholesterol accumulation in Kupffer cells and consequently reduced hepatic inflammation. ${ }^{1}$ Recently, also 25 -hydroxycholesterol $(25 \mathrm{HC})$, another oxysterol, was shown to correct the transport defect in NPC1 mutant cells. ${ }^{96}$ These data support the view that oxysterols can prevent and reduce lysosomal cholesterol accumulation in macrophages, thereby reducing the inflammatory response.

Finally, it was recently demonstrated that TG delivered to cultured macrophages as part of TG-rich particles, dramatically reduced lysosomal CE accumulation and improves lysosomal function, possibly by decreasing lysosomal $\mathrm{pH}$ and restoring lysosomal CE hydrolysis. ${ }^{97}$ Interestingly, it was shown that macrophages hydrolyze CE more efficiently when it is introduced into lysosomes of macrophages as a mixed CE and TG particle, compared to CE-containing particles alone. ${ }^{98}$ Knowing that TG-rich 
particles can be taken up by macrophage foam cells, thereby influencing the ability of foam cells to metabolize the overload of lysosomal CE, make these particles an interesting therapeutic option for multiple related disorders. Collectively, these findings are promising since so far lysosomal oxLDL has been shown to be highly resistant to removal. ${ }^{85,86}$

\section{Conclusion}

Recently, more evidence point towards the shared etiology of different obesityrelated diseases such as atherosclerosis and NASH. Whereas the presence of lipidladen macrophages is known to play a major role in these conditions, the exact molecular mechanisms remain unclear. Multiple studies suggest that the presence of lysosomal cholesterol accumulation in macrophages, and not the total amount of intracellular lipids is critical for the observed inflammatory response. So far, several mechanisms were shown to be involved in linking lysosomal cholesterol accumulation and inflammation. Future research should focus on mechanisms stimulating cholesterol transport out of the lysosomes, as increasing evidence is showing the importance of intracellular cholesterol distribution in relation to inflammation, thereby affecting multiple disorders. Although it is still possible that other forms of lipids are also involved, most evidence point towards the oxidized form of LDL as a major contributor to lysosomal cholesterol accumulation. Therefore, research should focus on the specific role of oxLDL in triggering inflammation due to lysosomal cholesterol trapping. Besides the stimulation of cholesterol transport out of the lysosomes, future research should focus on potential ways to block the uptake of oxLDL particles specifically in order to prevent accumulation of cholesterol in lysosomes. Furthermore, it should be investigated whether dietary intervention is a valid therapeutic option for reducing lysosomal cholesterol accumulation. Finally, to guarantee a systemic beneficial effect, it is recommended that studies will focus on the crosstalk between the different organs that are affected by lysosomal cholesterol accumulation. 


\section{References}

1. Bieghs V, Hendrikx T, van Gorp PJ, Verheyen F, Guichot YD, Walenbergh SM, Jeurissen ML, Gijbels M, Rensen SS, Bast A, Plat J, Kalhan SC, Koek GH, Leitersdorf E, Hofker MH, Lütjohann D, Shiri-Sverdlov R. The cholesterol derivative 27-hydroxycholesterol reduces steatohepatitis in mice. Gastroenterology. 2013;144:167-78 e1.

2. Bieghs V, Walenbergh SM, Hendrikx T, van Gorp PJ, Verheyen F, Olde Damink SW, Masclee AA, Koek $\mathrm{GH}$, Hofker MH, Binder CJ, Shiri-Sverdlov R. Trapping of oxidized LDL in lysosomes of Kupffer cells is a trigger for hepatic inflammation. Liver Int. 2013;33:1056-61.

3. Jerome WG, Cox BE, Griffin EE, Ullery JC. Lysosomal cholesterol accumulation inhibits subsequent hydrolysis of lipoprotein cholesteryl ester. Microsc Microanal. 2008;14:138-49.

4. Olefsky JM, Glass CK. Macrophages, inflammation, and insulin resistance. Annu Rev Physiol. 2010;72: 219-46.

5. Bhargava $\mathrm{P}$, Lee $\mathrm{CH}$. Role and function of macrophages in the metabolic syndrome. Biochem J. 2012; 442:253-62.

6. Wouters K, van Gorp PJ, Bieghs V, Gijbels MJ, Duimel H, Lütjohann D, Kerksiek A, van Kruchten R, Maeda N, Staels B, van Bilsen M, Shiri-Sverdlov R, Hofker MH. Dietary cholesterol, rather than liver steatosis, leads to hepatic inflammation in hyperlipidemic mouse models of nonalcoholic steatohepatitis. Hepatology. 2008;48:474-86.

7. Gerrity RG. The role of the monocyte in atherogenesis: II. Migration of foam cells from atherosclerotic lesions. Am J Pathol. 1981;103:191-200.

8. Sofer O, Fainaru M, Schafer Z, Goldman R. Regulation of lipoprotein lipase secretion in murine macrophages during foam cell formation in vitro. Effect of triglyceride-rich lipoproteins. Arterioscler Thromb. 1992;12:1458-66.

9. Goldstein JL, Brown MS. The LDL receptor. Arterioscler Thromb Vasc Biol. 2009;29:431-8.

10. Brown MS, Goldstein JL. A proteolytic pathway that controls the cholesterol content of membranes, cells, and blood. Proc Natl Acad Sci U S A. 1999;96:11041-8.

11. Brown MS, Dana SE, Goldstein JL. Cholesterol ester formation in cultured human fibroblasts. Stimulation by oxygenated sterols. J Biol Chem. 1975;250:4025-7.

12. Griffin EE, Ullery JC, Cox BE, Jerome WG. Aggregated LDL and lipid dispersions induce lysosomal cholesteryl ester accumulation in macrophage foam cells. J Lipid Res. 2005;46:2052-60.

13. Jerome WG, Cash C, Webber R, Horton R, Yancey PG. Lysosomal lipid accumulation from oxidized low density lipoprotein is correlated with hypertrophy of the Golgi apparatus and trans-Golgi network. J Lipid Res. 1998;39:1362-71.

14. Kunjathoor VV, Febbraio M, Podrez EA, Moore KJ, Andersson L, Koehn S, Rhee JS, Silverstein R, Hoff $\mathrm{HF}$, Freeman MW. Scavenger receptors class A-I/II and CD36 are the principal receptors responsible for the uptake of modified low density lipoprotein leading to lipid loading in macrophages. $J$ Biol Chem. 2002;277:49982-8.

15. Khoo JC, Miller E, McLoughlin P, Steinberg D. Enhanced macrophage uptake of low density lipoprotein after self-aggregation. Arteriosclerosis. 1988;8:348-58.

16. Couillard C, Ruel G, Archer WR, Pomerleau S, Bergeron J, Couture P, Lamarche B, Bergeron N. Circulating levels of oxidative stress markers and endothelial adhesion molecules in men with abdominal obesity. J Clin Endocrinol Metab. 2005;90:6454-9.

17. Weinbrenner T, Schröder H, Escurriol V, Fito M, Elosua R, Vila J, Marrugat J, Covas MI. Circulating oxidized LDL is associated with increased waist circumference independent of body mass index in men and women. Am J Clin Nutr. 2006;83:30-5; quiz 181-2.

18. Holvoet P, De Keyzer D, Jacobs DR, Jr. Oxidized LDL and the metabolic syndrome. Future Lipidol. 2008; 3:637-49.

19. Shoji T, Nishizawa Y, Fukumoto M, Shimamura K, Kimura J, Kanda H, Emoto M, Kawagishi T, Morii H. Inverse relationship between circulating oxidized low density lipoprotein (oxLDL) and anti-oxLDL antibody levels in healthy subjects. Atherosclerosis. 2000;148:171-7.

20. Holvoet P1, Kritchevsky SB, Tracy RP, Mertens A, Rubin SM, Butler J, Goodpaster B, Harris TB. The metabolic syndrome, circulating oxidized LDL, and risk of myocardial infarction in well-functioning elderly people in the health, aging, and body composition cohort. Diabetes. 2004;53:1068-73. 
21. Holvoet P, Vanhaecke J, Janssens S, Van de Werf F, Collen D. Oxidized LDL and malondialdehydemodified LDL in patients with acute coronary syndromes and stable coronary artery disease. Circulation. 1998;98:1487-94.

22. Lougheed M, Moore ED, Scriven DR, Steinbrecher UP. Uptake of oxidized LDL by macrophages differs from that of acetyl LDL and leads to expansion of an acidic endolysosomal compartment. Arterioscler Thromb Vasc Biol. 1999;19:1881-90.

23. Soccio RE, Breslow JL. Intracellular cholesterol transport. Arterioscler Thromb Vasc Biol. 2004;24: 1150-60.

24. Bieghs V, Verheyen F, van Gorp PJ, Hendrikx T, Wouters K, Lütjohann D, Gijbels MJ, Febbraio M, Binder CJ, Hofker MH, Shiri-Sverdlov R. Internalization of modified lipids by CD36 and SR-A leads to hepatic inflammation and lysosomal cholesterol storage in Kupffer cells. PLoS One. 2012;7:e34378.

25. Du H, Heur M, Witte DP, Ameis D, Grabowski GA. Lysosomal acid lipase deficiency: correction of lipid storage by adenovirus-mediated gene transfer in mice. Hum Gene Ther. 2002;13:1361-72.

26. Du H, Heur M, Duanmu M, Grabowski GA, Hui DY, Witte DP, Mishra J. Lysosomal acid lipase-deficient mice: depletion of white and brown fat, severe hepatosplenomegaly, and shortened life span. J Lipid Res. 2001;42:489-500.

27. Du H, Schiavi S, Wan N, Levine M, Witte DP, Grabowski GA. Reduction of atherosclerotic plaques by lysosomal acid lipase supplementation. Arterioscler Thromb Vasc Biol. 2004;24:147-54.

28. Li W, Yuan XM, Olsson AG, Brunk UT. Uptake of oxidized LDL by macrophages results in partial lysosomal enzyme inactivation and relocation. Arterioscler Thromb Vasc Biol. 1998;18:177-84.

29. Bieghs V, van Gorp PJ, Walenbergh SM, Gijbels MJ, Verheyen F, Buurman WA, Briles DE, Hofker MH, Binder CJ, Shiri-Sverdlov R. Specific immunization strategies against oxidized low-density lipoprotein: a novel way to reduce nonalcoholic steatohepatitis in mice. Hepatology. 2012;56:894-903.

30. Walenbergh SM, Koek GH, Bieghs V, Shiri-Sverdlov R. Non-alcoholic steatohepatitis: the role of oxidized low-density lipoproteins. J Hepatol. 2013;58:801-10.

31. Hoff HF, O'Neil J, Pepin JM, Cole TB. Macrophage uptake of cholesterol-containing particles derived from LDL and isolated from atherosclerotic lesions. Eur Heart J. 1990;11 Suppl E:105-15.

32. Haley NJ, Shio H, Fowler S. Characterization of lipid-laden aortic cells from cholesterol-fed rabbits. I. Resolution of aortic cell populations by metrizamide density gradient centrifugation. Lab Invest. 1977; 37:287-96.

33. Goldfischer S, Schiller B, Wolinsky H. Lipid accumulation in smooth muscle cell lysosomes im primate atherosclerosis. Am J Pathol. 1975;78:497-504.

34. Jerome WG, Lewis JC. Early atherogenesis in White Carneau pigeons. II. Ultrastructural and cytochemical observations. Am J Pathol. 1985;119:210-22.

35. Peters TJ, Muller M, De Duve C. Lysosomes of the arterial wall. I. Isolation and subcellular fractionation of cells from normal rabbit aorta. J Exp Med. 1972;136:1117-39.

36. Bieghs V, Walenbergh SM, Hendrikx T, van Gorp PJ, Verheyen F, Olde Damink SW, Masclee AA, Koek $\mathrm{GH}$, Hofker MH, Binder CJ, Shiri-Sverdlov R. Trapping of oxidized LDL in lysosomes of Kupffer cells is a trigger for hepatic inflammation. Liver Int. 2013;33:1056-61.

37. Choi AM, Ryter SW, Levine B. Autophagy in human health and disease. N Engl J Med. 2013;368: 65162.

38. Settembre C, Fraldi A, Jahreiss L, Spampanato C, Venturi C, Medina D, de Pablo R, Tacchetti C, Rubinsztein DC, Ballabio A. A block of autophagy in lysosomal storage disorders. Hum Mol Genet. 2008;17:119-29.

39. Settembre C, Fraldi A, Rubinsztein DC, Ballabio A. Lysosomal storage diseases as disorders of autophagy. Autophagy. 2008;4:113-4.

40. Raben N, Shea L, Hill V, Plotz P. Monitoring autophagy in lysosomal storage disorders. Methods Enzymol. 2009;453:417-49.

41. Singh R, Kaushik S, Wang Y, Xiang Y, Novak I, Komatsu M, Tanaka K, Cuervo AM, Czaja MJ. Autophagy regulates lipid metabolism. Nature. 2009;458:1131-5.

42. Koga H, Kaushik S, Cuervo AM. Altered lipid content inhibits autophagic vesicular fusion. Faseb J. 2010;24:3052-65.

43. Fraldi A, Annunziata F, Lombardi A, Kaiser HJ, Medina DL, Spampanato C, Fedele AO, Polishchuk R, Sorrentino NC, Simons K, Ballabio A. Lysosomal fusion and SNARE function are impaired by cholesterol accumulation in lysosomal storage disorders. Embo J. 2010; 29: 3607-20. 
44. Singh R, Xiang Y, Wang Y, Baikati K, Cuervo AM, Luu YK, Tang Y, Pessin JE, Schwartz GJ, Czaja MJ. Autophagy regulates adipose mass and differentiation in mice. J Clin Invest. 2009;119:3329-39.

45. Ost A, Svensson K, Ruishalme I, Brännmark C, Franck N, Krook H, Sandström P, Kjolhede P, Strålfors P. Attenuated mTOR signaling and enhanced autophagy in adipocytes from obese patients with type 2 diabetes. Mol Med. 2010;16:235-46.

46. Liao X, Sluimer JC, Wang Y, Subramanian M, Brown K, Pattison JS, Robbins J, Martinez J, Tabas I. Macrophage autophagy plays a protective role in advanced atherosclerosis. Cell Metab. 2012;15: 545-53.

47. Razani B, Feng C, Coleman T, Emanuel R, Wen H, Hwang S, Ting JP, Virgin HW, Kastan MB, Semenkovich CF. Autophagy links inflammasomes to atherosclerotic progression. Cell Metab. 2012;15:534-44.

48. Christian P, Sacco J, Adeli K. Autophagy: Emerging roles in lipid homeostasis and metabolic control. Biochim Biophys Acta. 2013;1831:819-24.

49. Yang L, Li P, Fu S, Calay ES, Hotamisligil GS. Defective hepatic autophagy in obesity promotes ER stress and causes insulin resistance. Cell Metab. 2010;11:467-78.

50. Hornung V, Latz E. Critical functions of priming and lysosomal damage for NLRP3 activation. Eur J Immunol. 2010;40:620-3.

51. Duewell P, Kono H, Rayner KJ, Sirois CM, Vladimer G, Bauernfeind FG, Abela GS, Franchi L, Nuñez G, Schnurr M, Espevik T, Lien E, Fitzgerald KA, Rock KL, Moore KJ, Wright SD, Hornung V, Latz E. NLRP3 inflammasomes are required for atherogenesis and activated by cholesterol crystals. Nature. 2010;464:1357-61.

52. Latz E. The inflammasomes: mechanisms of activation and function. Curr Opin Immunol. 2010;22: 28-33.

53. Zhou R, Yazdi AS, Menu P, Tschopp J. A role for mitochondria in NLRP3 inflammasome activation. Nature. 2011;469:221-5.

54. Tangirala RK, Jerome WG, Jones NL, Small DM, Johnson WJ, Glick JM, Mahlberg FH, Rothblat GH. Formation of cholesterol monohydrate crystals in macrophage-derived foam cells. J Lipid Res. 1994;35:93-104.

55. Nair PN, Sjogren U, Sundqvist G. Cholesterol crystals as an etiological factor in non-resolving chronic inflammation: an experimental study in guinea pigs. Eur J Oral Sci. 1998;106:644-50.

56. Rajamäki K, Lappalainen J, Oörni K, Välimäki E, Matikainen S, Kovanen PT, Eklund KK. Cholesterol crystals activate the NLRP3 inflammasome in human macrophages: a novel link between cholesterol metabolism and inflammation. PLoS One. 2010;5:e11765.

57. Gage J, Hasu M, Thabet M, Whitman SC. Caspase-1 deficiency decreases atherosclerosis in apolipoprotein E-null mice. Can J Cardiol. 2012;28:222-9.

58. Usui F, Shirasuna K, Kimura H, Tatsumi K, Kawashima A, Karasawa T, Hida S, Sagara J, Taniguchi S, Takahashi M. Critical role of caspase-1 in vascular inflammation and development of atherosclerosis in Western diet-fed apolipoprotein E-deficient mice. Biochem Biophys Res Commun. 2012;425:162-8.

59. Menu P, Pellegrin M, Aubert JF, Bouzourene K, Tardivel A, Mazzolai L, Tschopp J. Atherosclerosis in ApoE-deficient mice progresses independently of the NLRP3 inflammasome. Cell Death Dis. 2011;2:e137.

60. Zheng F, Xing S, Gong Z, Xing Q. NLRP3 Inflammasomes Show High Expression in Aorta of Patients with Atherosclerosis. Heart Lung Circ. 2013;22:746-50.

61. Dixon L, Berk M, Thapaliya S, Papouchado BG, Feldstein AE. Caspase-1-mediated regulation of fibrogenesis in diet-induced steatohepatitis. Lab Invest. 2012;92:713-23.

62. Dixon LJ, Flask CA, Papouchado BG, Feldstein AE, Nagy LE. Caspase-1 as a central regulator of high fat diet-induced non-alcoholic steatohepatitis. PLoS One. 2013;8:e56100.

63. Ioannou GN, Haigh WG, Thorning D, Savard C. Hepatic cholesterol crystals and crown-like structures distinguish NASH from simple steatosis. J Lipid Res. 2013;54:1326-34.

64. Conus S, Simon HU. Cathepsins and their involvement in immune responses. Swiss Med Wkly. 2010; 140: w13042.

65. Yang M, Sun J, Zhang T, Liu J, Zhang J, Shi MA, Darakhshan F, Guerre-Millo M, Clement K, Gelb BD, Dolgnov G, Shi GP. Deficiency and inhibition of cathepsin $K$ reduce body weight gain and increase glucose metabolism in mice. Arterioscler Thromb Vasc Biol. 2008;28:2202-8. 
66. Yang M, Zhang Y, Pan J, Sun J, Liu J, Libby P, Sukhova GK, Doria A, Katunuma N, Peroni OD, GuerreMillo M, Kahn BB, Clement K, Shi GP. Cathepsin L activity controls adipogenesis and glucose tolerance. Nat Cell Biol. 2007;9:970-7.

67. Sukhova GK, Shi GP, Simon DI, Chapman HA, Libby P. Expression of the elastolytic cathepsins S and K in human atheroma and regulation of their production in smooth muscle cells. J Clin Invest. 1998; 102:576-83.

68. Appelqvist $\mathrm{H}$, Waster $\mathrm{P}$, Kagedal $\mathrm{K}$, Ollinger $\mathrm{K}$. The lysosome: from waste bag to potential therapeutic target. J Mol Cell Biol. 2013;5:214-26.

69. Chwieralski CE, Welte T, Buhling F. Cathepsin-regulated apoptosis. Apoptosis. 2006;11:143-9.

70. Li W, Yuan XM. Increased expression and translocation of lysosomal cathepsins contribute to macrophage apoptosis in atherogenesis. Ann N Y Acad Sci. 2004;1030:427-33.

71. Droga-Mazovec G, Bojic L, Petelin A, Ivanova S, Romih R, Repnik U, Salvesen GS, Stoka V, Turk V, Turk B. Cysteine cathepsins trigger caspase-dependent cell death through cleavage of bid and antiapoptotic Bcl-2 homologues. J Biol Chem. 2008;283:19140-50.

72. Kornfeld S. Trafficking of lysosomal enzymes in normal and disease states. J Clin Invest. 1986;77:1-6.

73. Porter K, Lin Y, Liton PB. Cathepsin B is up-regulated and mediates extracellular matrix degradation in trabecular meshwork cells following phagocytic challenge. PLoS One. 2013;8:e68668.

74. Liu J, Sukhova GK, Yang JT, Sun J, Ma L, Ren A, Xu WH, Fu H, Dolganov GM, Hu C, Libby P, Shi GP. Cathepsin $L$ expression and regulation in human abdominal aortic aneurysm, atherosclerosis, and vascular cells. Atherosclerosis. 2006;184:302-11.

75. Lutgens SP, Cleutjens KB, Daemen MJ, Heeneman S. Cathepsin cysteine proteases in cardiovascular disease. Faseb J. 2007;21:3029-41.

76. Jormsjö S, Wuttge DM, Sirsjö A, Whatling C, Hamsten A, Stemme S, Eriksson P. Differential expression of cysteine and aspartic proteases during progression of atherosclerosis in apolipoprotein E-deficient mice. Am J Pathol. 2002;161:939-45.

77. Lutgens $E$, Lutgens SP, Faber BC, Heeneman S, Gijbels MM, de Winther MP, Frederik P, van der Made I, Daugherty A, Sijbers AM, Fisher A, Long CJ, Saftig P, Black D, Daemen MJ, Cleutjens KB. Disruption of the cathepsin $\mathrm{K}$ gene reduces atherosclerosis progression and induces plaque fibrosis but accelerates macrophage foam cell formation. Circulation. 2006;113:98-107.

78. Sukhova GK, Zhang Y, Pan JH, Wada Y, Yamamoto T, Naito M, Kodama T, Tsimikas S, Witztum JL, Lu ML, Sakara Y, Chin MT, Libby P, Shi GP. Deficiency of cathepsin S reduces atherosclerosis in LDL receptor-deficient mice. J Clin Invest. 2003;111:897-906.

79. Kitamoto S, Sukhova GK, Sun J, Yang M, Libby P, Love V, Duramad P, Sun C, Zhang Y, Yang X, Peters C, Shi GP. Cathepsin L deficiency reduces diet-induced atherosclerosis in low-density lipoprotein receptor-knockout mice. Circulation. 2007;115:2065-75.

80. Hakala JK, Oksjoki R, Laine P, Du H, Grabowski GA, Kovanen PT, Pentikäinen MO. Lysosomal enzymes are released from cultured human macrophages, hydrolyze LDL in vitro, and are present extracellularly in human atherosclerotic lesions. Arterioscler Thromb Vasc Biol. 2003;23:1430-6.

81. Feldstein AE, Werneburg NW, Canbay A, Guicciardi ME, Bronk SF, Rydzewski R, Burgart LJ, Gores GJ. Free fatty acids promote hepatic lipotoxicity by stimulating TNF-alpha expression via a lysosomal pathway. Hepatology. 2004;40:185-94.

82. Li Z, Berk M, McIntyre TM, Gores GJ, Feldstein AE. The lysosomal-mitochondrial axis in free fatty acidinduced hepatic lipotoxicity. Hepatology. 2008;47:1495-503.

83. Moles A, Tarrats N, Fernandez-Checa JC, Mari M. Cathepsins B and D drive hepatic stellate cell proliferation and promote their fibrogenic potential. Hepatology. 2009;49:1297-307.

84. Yancey PG, Jerome WG. Lysosomal cholesterol derived from mildly oxidized low density lipoprotein is resistant to efflux. J Lipid Res. 2001;42:317-27.

85. Dhaliwal BS, Steinbrecher UP. Cholesterol delivered to macrophages by oxidized low density lipoprotein is sequestered in lysosomes and fails to efflux normally. $J$ Lipid Res. 2000;41:1658-65.

86. Lougheed M, Zhang HF, Steinbrecher UP. Oxidized low density lipoprotein is resistant to cathepsins and accumulates within macrophages. J Biol Chem. 1991;266:14519-25.

87. Jerome WG, Lewis JC. Early atherogenesis in White Carneau pigeons: effect of a short-term regression diet. Exp Mol Pathol. 1990;53:223-38. 
88. Yancey PG, Miles S, Schwegel J, Jerome WG. Uptake and trafficking of mildly oxidized LDL and acetylated LDL in THP-1 cells does not explain the differences in lysosomal metabolism of these two lipoproteins. Microsc Microanal. 2002;8:81-93.

89. Subramanian K, Balch WE. NPC1/NPC2 function as a tag team duo to mobilize cholesterol. Proc Natl Acad Sci U S A. 2008;105:15223-4.

90. Liu B, Ramirez CM, Miller AM, Repa JJ, Turley SD, Dietschy JM. Cyclodextrin overcomes the transport defect in nearly every organ of NPC1 mice leading to excretion of sequestered cholesterol as bile acid. J Lipid Res. 2010;51:933-44.

91. Abi-Mosleh L, Infante RE, Radhakrishnan A, Goldstein JL, Brown MS. Cyclodextrin overcomes deficient lysosome-to-endoplasmic reticulum transport of cholesterol in Niemann-Pick type C cells. Proc Natl Acad Sci U S A. 2009;106:19316-21.

92. Taylor AM, Liu B, Mari Y, Repa JJ. Cyclodextrin mediates rapid changes in lipid balance in Npc1-/- mice without carrying cholesterol through the bloodstream. J Lipid Res. 2012;53:2331-42.

93. Griffin LD, Gong W, Verot L, Mellon SH. Niemann-Pick type C disease involves disrupted neurosteroidogenesis and responds to allopregnanolone. Nat Med. 2004;10:704-11.

94. Liao G, Cheung S, Galeano J, Ji AX, Qin Q, Bi X. Allopregnanolone treatment delays cholesterol accumulation and reduces autophagic/lysosomal dysfunction and inflammation in Npc1-/- mouse brain. Brain Res. 2009;1270:140-51.

95. Frolov A, Zielinski SE, Crowley JR, Dudley-Rucker N, Schaffer JE, Ory DS. NPC1 and NPC2 regulate cellular cholesterol homeostasis through generation of low density lipoprotein cholesterol-derived oxysterols. J Biol Chem. 2003;278:25517-25.

96. Ohgane K, Karaki F, Dodo K, Hashimoto Y. Discovery of oxysterol-derived pharmacological chaperones for NPC1: implication for the existence of second sterol-binding site. Chem Biol. 2013;20:391-402.

97. Ullery-Ricewick JC, Cox BE, Griffin EE, Jerome WG. Triglyceride alters lysosomal cholesterol ester metabolism in cholesteryl ester-laden macrophage foam cells. J Lipid Res. 2009;50:2014-26.

98. Mahlberg FH, Glick JM, Jerome WG, Rothblat GH. Metabolism of cholesteryl ester lipid droplets in a J774 macrophage foam cell model. Biochim Biophys Acta. 1990;1045:291-8. 
General discussion 
Chapter 9 


\section{General discussion}

Despite the strong association demonstrated between lysosomal cholesterol accumulation in Kupffer cells (KCs) and hepatic inflammation, a causal link was never investigated. In the present thesis, we aimed to establish a causal relationship between lysosomal cholesterol accumulation inside $\mathrm{KCs}$ and hepatic inflammation. Furthermore, we translated the knowledge obtained from animal models to human $\mathrm{NASH}$. The major findings of these studies will be discussed and placed in the context of current knowledge.

\section{Lysosomal cholesterol storage inside Kupffer cells is causally related to hepatic inflammation}

Under healthy conditions, lipoproteins, such as low-density lipoproteins (LDL), are taken up by macrophages. After various processes, the LDL particles are remodeled into free cholesterol, hereby facilitating further clearance out of the cell. ${ }^{1}$ Under these circumstances, the size of the macrophage remains similar and the amount of circulating LDL is low. However, in obese subjects, plasma LDL level concentrations are elevated. ${ }^{2,3}$ In the presence of high LDL levels, LDL-cholesterol is scavenged by macrophages in an unregulated manner leading to a foamy macrophage appearance. These foamy macrophages are known to play an important role in triggering inflammation during atherosclerosis. ${ }^{4,5}$ Recently, the interrelationship between NASH and atherosclerosis becomes more evident. ${ }^{6}$ In line, bloated foamy $\mathrm{KCs}$, resembling the foamy macrophages present in atherosclerotic lesions, were also observed in livers of hyperlipidemic mice ${ }^{7}$ and human NASH patients. ${ }^{8}$ Strikingly, additional research clearly demonstrated that hepatic inflammation was independent of the size of the KCs. ${ }^{9}$ These data suggested it is not the total cholesterol amount inside the KCs that trigger hepatic inflammation, but rather the disturbed cholesterol distribution within the KCs. This suggestion is also potentially relevant to the field of atherosclerosis, since only in vitro studies showed a link between cholesterol distribution inside macrophages and vascular inflammation and was never tested in vivo.

When LDL lipoproteins are taken up by macrophages, these are initially directed to the lysosomes for further processing. After hydrolysis by lysosomal enzymes, the lipoproteins are transferred into the cytoplasm via so-called Niemann-Pick type C1 (NPC1) proteins. However, in foam cells as present in the atherosclerotic lesions, cholesterol is resistant to efflux into the cytoplasm and instead, accumulates in the lysosomes of macrophages. ${ }^{10-13}$ Likewise, we recently showed cholesterol accumulation mainly inside lysosomes of KCs in the livers of hyperlipidemic mice. ${ }^{14}$ Relevantly, in lysosomal storage diseases, lysosomal cholesterol accumulation in macrophages is associated with inflammatory responses ${ }^{15-17}$ and hepatic inflammation 
(Chapter 3). However, these studies in the context of lysosomal storage diseases and inflammation merely provided evidence for an association between lysosomal cholesterol accumulation and the inflammatory response and lacked causality. We next hypothesized that lysosomal cholesterol accumulation in KCs is the actual trigger for the development of hepatic inflammation. For this purpose, we used mice with a loss-of-function mutation in the NPC1 gene. This single mutation in the NPC1 gene causes excessive lysosomal storage of cholesterol and affects correct lysosomal function. ${ }^{18}$ As a tool to induce lysosomal cholesterol accumulation specifically inside $\mathrm{KCs}$, we transplanted NPC1 mutant (NPC1 ${ }^{\text {mut }}$ ) bone marrow into $\mathrm{Ldlr}^{-/-}$mice in Chapter 4. In line with our expectations, compared to the wildtype-transplanted (tp) mice, the NPC $1^{\text {mut }}$-tp mice demonstrated dramatically enlarged macrophages due to macrophage granuloma formation, elevated lysosomal enzyme levels, increased hepatic inflammation and fibrosis. To conclude, we demonstrated that lysosomal cholesterol accumulation inside $\mathrm{KCS}$ is the trigger for hepatic inflammation. Moreover, the excessive cholesterol storage inside lysosomes affects lysosomal function through lysosomal enzyme disturbances.

\section{The oxidized fraction of LDL inside lysosomes of Kupffer cells contributes substantially to the hepatic inflammatory response}

Unlike acetylated LDL (acLDL), cell culture studies clearly demonstrated that treatment with specifically the oxidized lipoprotein fraction resulted in lysosomal cholesterol accumulation. ${ }^{19-21}$ Once inside the lysosomes, the oxLDL-derived cholesterol fraction is resistant for efflux and starts to accumulate. ${ }^{20}$ Further data reveals the presence of functional lysosomal acid lipase inside foam cells, and herewith excludes lysosomal enzyme defects during intracellular oxLDL accumulation. ${ }^{22}$ However, these studies were all performed in an in vitro setting and were, so far, not been demonstrated in vivo. Therefore, we next hypothesized a similar association between lysosomal oxLDL accumulation and hepatic inflammation in vivo. Upon injection of several lipoprotein species in $\mathrm{Ldll}^{-/-}$mice, we demonstrated in Chapter 3 that, unlike native LDL and acLDL, it is oxLDL that tends to accumulate inside lysosomes of KCs. In the same chapter, we showed that the increased lysosomal cholesterol accumulation inside KCs was linked to a higher hepatic inflammatory response in $\mathrm{Ldll}^{-/}$mice. To further elucidate the specific contribution of lysosomal oxLDL accumulation to hepatic inflammation, we induced the levels of anti-oxLDL antibodies in NPC1 ${ }^{\text {mut }}$-tp mice (Chapter 4) and in Ldlr $^{-/}$mice (Chapter 5). These antioxLDL antibodies have been shown to block the uptake of oxLDL by macrophages, and thus prevent oxLDL from being internalized and directed to lysosomes. ${ }^{23}$ In the presence of high anti-oxLDL antibodies, NPC1 ${ }^{\text {mut }}$-tp mice demonstrated significant improvements in cholesterol metabolism, lysosomal function, hepatic inflammation and fibrosis compared to NPC1 ${ }^{\text {mut }}$-tp mice without treatment. In line, high anti-oxLDL IgM titers in $\mathrm{Ldlr}^{-/}$mice displayed a reduced foamy KC appearance, hepatic 
inflammation and fibrosis. These results are overwhelming since, even though the oxLDL levels are higher in patients with the metabolic syndrome (MetS), the percentage of oxLDL relative to LDL in MetS patients is still considered to be very low $(0.6-1.8 \%) .{ }^{24}$ In NASH patients, it has been found that plasma oxLDL is only 1.4 times higher than the average oxLDL level detected in control subjects. ${ }^{25}$ Therefore, the improvements in liver and lysosomes after preventing oxLDL uptake by pneumococcal immunization exceeded our expectations and point towards the important contribution of oxLDL in hepatic inflammation. Apart from oxLDL, other modified lipids such as aggregated LDL (aggLDL) and cholesteryl-ester rich lipid dispersions (DISP) may also lead to cholesterol accumulation inside lysosomes and sustained inhibition of hydrolysis and subsequent efflux. ${ }^{10,26}$ In addition, sphingomyelin-rich particles were shown to be highly present inside lysosomes of macrophages after incubation with different lipoprotein species. ${ }^{27}$ To note, these lipids (i.e. aggLDL, DISP and sphingomyelin) were not studied in these experiments and could provide an explanation for the fact that hepatic inflammation in immunized NPC1 ${ }^{\text {mut }}$-tp mice was not completely abolished to control values.

\section{Lysosomes and lysosomal enzymes play a central role in NASH}

With the help of lysosomal enzymes, lysosomes are best known for its primary role in protein degradation. However, lysosomal function is not merely restricted to degradation of proteins, but increasing evidence now demonstrates that the lysosomal compartment plays also a role in the immune system and can be seen as secretory vesicles. ${ }^{28,29}$ Lysosomes are able to secrete its content, including lysosomal enzymes, via fusion with the plasma membrane. Cathepsins, a specific class of lysosomal enzymes, have been shown to be significantly involved in mediating the inflammatory response and cholesterol trafficking. ${ }^{30-32}$ Consequently, diseases that are predominantly characterized by inflammation and excessive cholesterol storage inside macrophages, such as atherosclerosis and lysosomal storage diseases (NPC1 disease), were linked to modified levels of cathepsins. For example, several cathepsins were abundantly expressed and secreted in atherosclerotic vessels. ${ }^{33,34}$ Mice used to study atherosclerosis showed a similar increase in lysosomal cathepsins inside atherosclerotic lesions, specifically in macrophages and/or lipid-rich areas. ${ }^{35}$ In addition, deficiency of cathepsins in mice led to a reduction in atherosclerotic development. $^{32,36,37}$ In line with these data obtained from atherosclerosis, modified levels of cathepsins were also observed in lysosomal storage diseases. Levels of several cathepsins (cathepsin B, D and S) were found to be modified in the livers and brains of $\mathrm{NpC1}^{-1-}$ mice and NPC1 patients. ${ }^{38-41}$ Changes of cathepsins in liver and brain could be reflected by cathepsin measurements in plasma and detected modified plasma cathepsin levels in NPC1 patients compared to control. ${ }^{39,41}$ As a result, these researchers proposed plasma cathepsin D as a potential biomarker for NPC1 disesase. Similar to these findings, we have demonstrated plasma cathepsin $D$ as a diagnostic 
tool to detect NASH in children and adults in the current thesis (Chapters 6 \& 7). Cathepsin release was also found to play an important role in the development of liver disease. Alterations of other cathepsins, including cathepsin $D$, were detected in liver specimens of NAFLD and cirrhotic patients compared to controls. ${ }^{42,43}$ Similarly, we have recently shown that lysosomal cholesterol storage inside KCs of mice correlated with a changed hepatic activity of cathepsin D and an altered hepatic gene expression of a whole set of cathepsins (Chapter 4). ${ }^{14}$ These data indicate that lysosomal cathepsins are involved in the development of metabolic diseases underlying lysosomal cholesterol accumulation and inflammation. Little is known about the mechanisms that induce lysosomal enzyme relocation. It has been proposed that it is specifically the uptake of oxLDL that may lead to rupture and subsequent leakage of lysosomal enzymes (Chapter 8)..$^{44,45}$ Indeed, cellular incubation with oxLDL showed sustained secretion of pro-cathepsin D. ${ }^{46}$ In turn, cytosolic cathepsins are important mediators of the caspase-mediated apoptotic pathway. ${ }^{47-49}$ Additionally, cytosolic cathepsins are able to activate the inflammasome, thereby potentially triggering hepatic inflammation (Chapter 8). ${ }^{50}$ Thus, these findings provide a possible mechanism by which lysosomal oxLDL accumulation specifically could lead to modified cathepsin levels.

\section{NASH and Niemann-Pick disease type C1 share disease mechanisms}

The liver holds responsibility for a healthy lipid profile and particularly governs cholesterol homeostasis. As a result, the liver is the primary affected site during metabolic disorders characterized by a cholesterol imbalance including atherosclerosis, NASH and lysosomal cholesterol storage disorders, particularly the socalled Niemann-Pick Type C1 disease. The latter disorder is characterized by an excessive storage of cholesterol inside lysosomes of macrophages and therefore has a progressive course of disease. Typical clinical manifestations for NPC1 disease include neurological problems (i.e. loss of motor skills and mental regression) and (hepato)splenomelagy. ${ }^{51}$ In Chapter 3, 4 and 5, we demonstrated lysosomal cholesterol accumulation inside KCs as the main underlying mechanism during NASH. Although to a much lesser extent, this feature resembles the primary characteristic of NPC1 disease and raises the possibility of a shared disease mechanism between NASH and NPC1 disease. The same holds true for atherosclerosis, whereby it was postulated that atherosclerosis has features of an acquired lysosomal storage disorder and accelerated more rapidly in the absence of the NPC1 protein. ${ }^{52,53}$ In addition to the pathogenesis of NASH, NPC1 disease also resulted in liver inflammation and apoptosis. ${ }^{18}$ Apoptosis is a typical hallmark of NASH and develops rather at a late stage, upon 3 months of a high-fat, high-cholesterol diet, in a mouse model for NASH. ${ }^{54}$ Since the magnitude of lysosomal cholesterol accumulation in the liver of NPC1 patients is more progressive, apoptosis in mice with NPC1 disease is manifested already at an early age around 9 weeks at regular chow. ${ }^{18,55,56}$ In addition to 
inflammation and apoptosis, oxidative stress is also a possible underlying mechanism linking NASH and NPC1 disease. Multiple studies demonstrated that oxidative stress is central to NPC1 pathology. ${ }^{53,57-60}$ In human NPC1 ${ }^{\text {mut }}$ fibroblasts, proteins related to oxidative stress were shown to be differentially expressed. ${ }^{61}$ These observations could be translated to humans, whereby an increased amount of oxidation products were detected in plasma of NPC1 patients. ${ }^{62,63}$ Similar to NPC1 patients, NASH patients also displayed higher plasma lipid peroxidation products, including oxLDL and thioredoxin. ${ }^{25,64}$ Although NPC1 was exclusively deficient in the hematopoietic system and does not fully represent the complete NPC1 knockout, our results underline these findings, whereby preventing oxidized cholesterol mice from being internalized into $\mathrm{KCs}$ of NPC1 ${ }^{\text {mut }}$-tp mice resulted in liver improvements (Chapter 4). Applying the same method to $\mathrm{Ldlr}^{-/}$mice in Chapter 5 demonstrated similar improvements in the liver. To study lysosomal function, we analyzed lysosomal enzymes in human NASH patients. In Chapter 7, we clearly demonstrated elevated levels of cathepsins in plasma of adult NASH patients, which reduced upon NASH regression. Likewise, cathepsins were found to be higher in $\mathrm{Npc1}^{-/}$mouse plasma, liver and brain, which decreased upon cholesterol-lowering treatment. ${ }^{39}$ Similar to Chapters 6 \& 7, particularly plasma cathepsin D was suggested as a potential biomarker for NPC1 disease. ${ }^{41}$ In addition to the common underlying mechanisms of these diseases, therapies that are currently under investigation for NPC1 disease have similar targets to those of NASH and include lipid-lowering drugs, ${ }^{65-67}$ anti-oxidants/calciummodulators $^{68}$ and anti-inflammatory drugs. ${ }^{69}$ In line with this concept, we used 27-hydroxycholesterol, a cholesterol derivative that was shown to improve cholesterol metabolism in fibroblasts of patients with NPC1 disease, as a method to reduce hepatic inflammation in mice. ${ }^{14}$ Ideally, we suggest that future therapies should be aimed at reducing lysosomal cholesterol accumulation in both NASH and NPC1 disease. In conclusion, our data point towards NASH and NPC1 disease as two diseases that share one central underlying mechanism being lysosomal cholesterol accumulation.

\section{A distinct pathophysiology exists between NASH in children and adults}

In the search for the molecular mechanism by which lysosomal enzymes contribute to hepatic inflammation, we observed striking differences between NASH in children and adults. Whereas, we observed a decrease of plasma cathepsin D in NASH children (Chapter 6), we have found an increase of cathepsin D in the plasma of adults with NASH (Chapter 7). These findings suggest a difference in disease pathology between NASH in adults and in children. To our knowledge, this is the first time a difference between NASH in children and adults is reflected in plasma. So far, the reason for this discrepancy is unknown. The difference in pathophysiology between NASH in children and adults could be due to age-dependent changes of the lysosomal compartment. 
Indeed, lysosomal function has previously been shown to be an age-dependent process, whereby the amount of lysosomes increases upon ageing. ${ }^{70-72}$ These data suggest that at a young age, the amount of lysosomes is low and consequently the cells have difficulties to cope with excessive amounts of cholesterol. As such, upon high-cholesterol circumstances, the stability of the lysosomal membrane is likely to decrease ${ }^{73}$, which can eventually lead to lysosomal rupture and subsequent lysosomal enzyme release into the cytosol. Lysosomal enzyme secretion into the plasma is hereby prevented and instead, the cytosolic lysosomal enzymes activate the apoptotic signaling pathway inducing rapid cell death. ${ }^{74}$ This mechanism could explain the reduction of cathepsin D in the plasma of pediatric subjects with NASH compared to subjects without liver inflammation (Chapter 6). In contrast to young cells, older cells possess a higher number of lysosomes ${ }^{70-72}$, suggesting increased cholesterol storage capabilities inside the cells. It is therefore likely that adult lysosomes can cope better with the cholesterol accumulation and would be less likely to rupture. Nevertheless, cholesterol-filled lysosomes have been shown to induce disturbances in the lysosomal enzyme trafficking pathway ${ }^{75,76}$ leading to increased levels of lysosomal enzymes in plasma. ${ }^{77,78}$ Plasma LDL oxidation can be viewed as a representative parameter of oxidative stress and has shown to be induced in healthy elderly compared to young controls and therefore is strongly associated with the course of ageing. ${ }^{79,80}$ In vitro, particularly the intracellular accumulation of the oxidized cholesterol fraction (oxLDL) has been shown to enhance extracellular secretion of pro-cathepsin D. ${ }^{44,46}$ Altogether, the observed differences between NASH in children and adults could be explained by the fact that secretion of cathepsin $D$ into the plasma is dependent on age-related changes of the lysosomes and is dependent on accumulation of specific lipid species. In line, several studies indeed describe that the histological and pathological characteristics of NASH are age-dependent. ${ }^{81-84}$ Further studies are warranted to precisely determine the contribution of lysosomes to NASH disease pathology in children and adults.

\section{Storage solutions: novel ways for the detection and inhibition of non- alcoholic steatohepatitis}

The currently available non-invasive diagnostic methods for the detection of NASH are poor and lack specificity. Consequently, no approved therapy against NASH exists. This situation is a key clinical problem as NASH patients need close monitoring and regular follow-up.

\section{Diagnostics}

The gold standard to detect NASH is still the histological assessment of a liver biopsy. Due its invasive procedure, patients experience discomfort, stress and pain and run the risk of suffering from severe complications such as internal bleedings. Noninvasive ways to diagnose NASH include plasma measurements of aminotransferases 
(ALT, AST) and gamma-glutamyl transpeptidase (GGT). However, these enzymes have a poor clinical utility in diagnosing $\mathrm{NASH}^{85}$ Another promising marker for the diagnosis of $\mathrm{NASH}$, which is currently under extensive investigation, is the liver enzyme cytokeratin-18 (CK-18). Whilst some studies demonstrated that CK-18 has the potential to differentiate NASH patients from subjects with steatosis ${ }^{86-90}$, others could not reach similar results. ${ }^{91,92}$ Altogether, a major disadvantage of using these noninvasive tools is the lack of specificity and sensitivity to differentiate between patients with steatosis and NASH subjects. Additionally, instead of early indicators of disease, these markers are associated with liver function and apoptosis, thus, are more representative for late stages of liver disease. Therefore, an accurate non-invasive marker to detect NASH is urgently needed.

The identification of oxLDL as one of the leading triggers for hepatic inflammation (Chapters 2, 3, 4 and 5) could contribute to novel non-invasive diagnostic methods for NASH. Chalasani et al demonstrated elevated levels of circulatory plasma oxLDL in NASH patients ${ }^{25}$, indicating that plasma oxLDL should be monitored in patients with hepatic inflammation. In line with plasma oxLDL, naturally occurring IgM antibody responses to oxLDL correlated negatively with NASH (Chapter 4 and 5). In this view, the level of plasma anti-oxLDL IgM antibodies could be a useful predictor for hepatic inflammation. Although, it has to be taken into account that basal IgM antibodies may differ naturally between people and can very over time. ${ }^{93,94}$ Moreover, since plasma cathepsin D correlates with histological classifications of NAFLD in children and adults and responds to intervention (Chapter $6 \& 7$ ), it is suggested that plasma cathepsin D should be monitored as a possible biomarker for hepatic inflammation. Also other plasma lysosomal enzymes in the context of NASH should be further studied in the future. Altogether, the data of this thesis suggest that plasma oxLDL, anti-oxLDL IgM antibodies and cathepsin D, either alone or in combination, could be used as noninvasive markers for the detection of NASH. All of the abovementioned potential markers should be tested for its clinical utility in large, human NASH cohorts.

\section{Therapy}

Early detection of NASH is important in order to apply adequate therapy and preventing NASH from further progression into irreversible liver disease. Unfortunately, NASH patients most commonly experience no symptoms making early detection of this disease almost impossible. If left untreated, NASH could progress to chronic irreversible liver disease, leaving a liver transplantation as the only solution available.

In the current thesis (Chapter 3, 4 and 5), lysosomal cholesterol accumulation inside KCs was identified as one the main causes for hepatic inflammation. Therefore, methods to modulate lysosomal cholesterol storage in KCs may be effective for treating NASH. One example is the exogenous administration of 27-hydroxycholesterol, a cholesterol derivative that was shown to improve cholesterol 
metabolism in fibroblasts of patients with NPC1 disease, to $\mathrm{Ldlr}^{-/}$mice. Application of $27 \mathrm{HC}$ to these mice could both prevent as well as treat $\mathrm{NASH}^{14}$ and has opened up a new avenue of testing other oxysterols with similar effects. ${ }^{95}$ Another possibility to overcome lysosomal cholesterol accumulation is to improve intracellular trafficking of cholesterol $^{96}$, for example via NPC1 overexpression. Methods to reduce lysosomal cholesterol accumulation in macrophages could not merely be applicable to NASH, but could also be beneficial for NPC1 disease, and should be tested. An indirect way to reduce lysosomal cholesterol accumulation inside macrophages is the use of cholesterol-lowering drugs. In parallel with lowering LDL levels, these drugs are able to decrease the susceptibility of LDL oxidation. ${ }^{97,98}$ Fibrates are well-recognized lipidlowering agents, however, human studies showed little or no effect on NASH histology or conclusions were based on liver aminotransferase levels solely. ${ }^{99,100}$ While statins have proven to reduce the risk for coronary and vascular events, the effect on NASH is not yet fully known. Small preliminary studies have shown that the use of statins at a low-dose is rather safe, well-tolerated and could possibly improve NAFLD histology. However, convincing histological data showing improvements of NASH histology is clearly lacking. As such, further research is warranted to test the efficacy of statins in large, biopsy-proven human NASH cohorts with a long duration time in order to draw correct conclusions on the use of statins on NASH patients. ${ }^{101}$

In the current thesis, lysosomal storage of specifically oxLDL was identified as an important contributor to hepatic inflammation. Therefore, drugs directed at reducing oxLDL would be of great importance as therapeutic intervention against NASH. One way to reduce lipid peroxidation is the use of anti-oxidants. As such, anti-oxidants have been shown effective in improving histological features of NASH patients and included vitamin $E^{102}$, betaine ${ }^{103}$, pentoxyfilline ${ }^{104}$, and the combination ursodeoxycholic acid with vitamin E. ${ }^{105}$ However, high dosages of vitamin $E$ have been shown to be associated with increased mortality rates and increased risk of prostate cancer in elderly men. ${ }^{106,107}$ Another potential intervention strategy against NASH is the use of a vaccination protocol with heat-inactivated pneumococci in order to prevent the development of NASH (Chapters $4 \& 5$ ). Notion has to be taken that infections with S. Pneumoniae frequently occur, and that pneumococcal vaccines are already being used in clinical practice. Therefore, it would be interesting to investigate the potential effect of these infections/vaccines on NASH pathogenesis in humans. Apart from NASH, the pneumococcal immunization method has also been shown beneficial for other inflammatory diseases characterized with elevated oxLDL levels, including atherosclerosis. ${ }^{23}$ Besides an active immunization approach, a passive immunization method of anti-oxLDL IgM antibodies and anti-phosphorylcholine antibodies was successfully tested to reduce inflammatory diseases ${ }^{108,109}$ and holds promise in reducing NASH. Altogether, potential new therapies against NASH that could be derived from this thesis are therapies that are aimed at reducing lysosomal cholesterol accumulation, lipid peroxidation and preventing oxLDL uptake into KCs. 


\section{Novel findings of this thesis}

Despite the strong association demonstrated between lysosomal cholesterol accumulation and hepatic inflammation, a causal link was never investigated. In the present thesis, we aimed to establish the causality between lysosomal oxLDL accumulation inside KCs and hepatic inflammation. The novel findings of this thesis are presented below.

- OxLDL possesses harmful and inflammatory aspects. (Chapter 2)

- Lysosomal cholesterol accumulation inside KCs is the trigger for the development of NASH. (Chapters 3, 4 and 5)

- Lysosomal cholesterol inside KCs, particularly the oxidized cholesterol, significantly contributes to hepatic inflammation. (Chapters 2, 3, 4 and 5)

- NASH and NPC1 disease have overlapping disease mechanisms. (Chapters 3, 4 and 5)

- Naturally occurring anti-oxLDL IgM antibodies can bind to oxLDL and prevent the development of NASH. (Chapter 5)

- $\quad$ Plasma cathepsin D is able to predict pediatric hepatic inflammation. (Chapter 6)

- Plasma cathepsin D could potentially be used as a non-invasive method to follow NASH progression and regression in adults. (Chapter 7)

- A possible distinct pathophysiology exists between NASH in children and adults. (Chapters 6 \& 7)

- Lysosomes and lysosomal enzymes are central to NASH disease pathology. (Chapters 2 and 8)

- Lysosomal oxLDL accumulation can trigger NASH and atherosclerosis through release of cytosolic and extracellular cathepsins. (Chapter 8)

\section{List of suggested non-invasive markers for NASH diagnosis}

- Plasma oxLDL

- Naturally occurring anti-oxLDL IgM antibodies

- Plasma cathepsin D

\section{List of possible therapy options against NASH}

- $\quad$ Lipid-lowering drugs

- Methods aimed at modulation of lysosomal cholesterol accumulation

- Anti-oxidants

- Pneumococcal immunization 
A

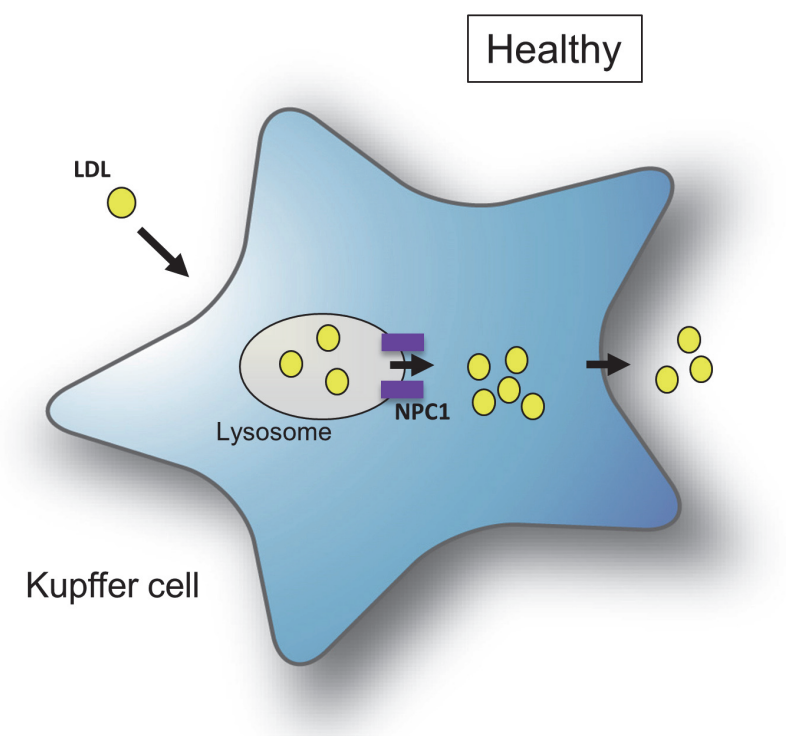

B

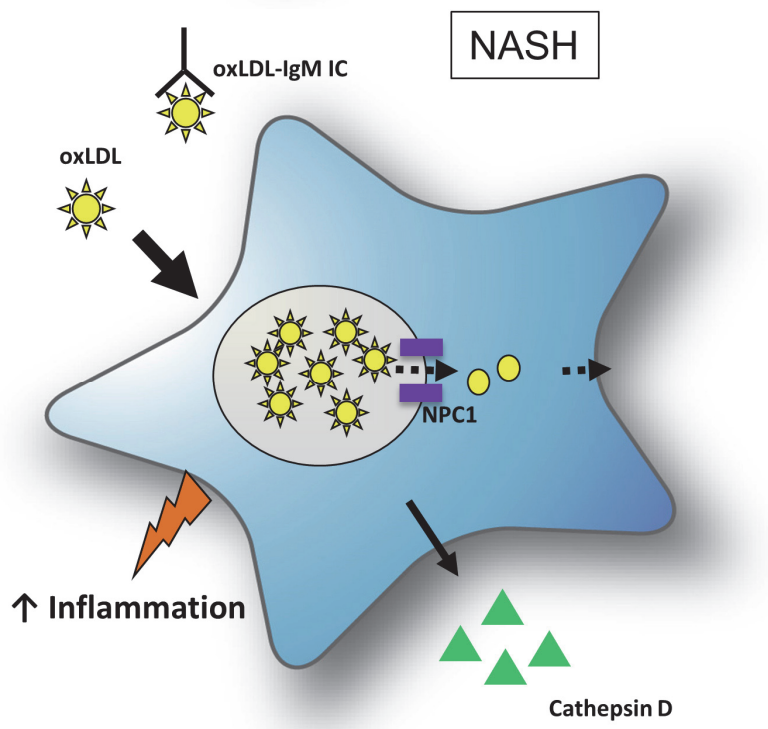

Summary of the main findings of this thesis. (A) Under normal conditions, LDL is internalized by Kupffer cells (KCS) and directed to the lysosomes. After hydrolysation by lysosomal enzymes, free cholesterol is subsequently transported into the cytoplasm via the cholesterol transporters Niemann-Pick type C1 (NPC1) proteins and cleared by cholesterol efflux mechanisms, or secreted into bile. (B) Unlike LDL, oxLDL could be scavenged by specific IgM anti-oxLDL antibodies or can be internalized by KCs and directed to the lysosomes during NASH. Rather than transport to the cytoplasm, oxLDL has been shown to be resistant for efflux out of the lysosome and starts to accumulate, hereby triggering hepatic inflammation. Further cholesterol transport via NPC1 proteins is abrogated and lysosomes become enlarged from the excessive amounts of oxLDL storage. As a result, either lysosomes may rupture and release cathepsins into the cytosol or lysosomes suffer from trafficking defects hereby leading to enhanced extracellular secretion of cathepsins. 


\section{References}

1. Ghosh S. Macrophage cholesterol homeostasis and metabolic diseases: critical role of cholesteryl ester mobilization. Expert Rev Cardiovasc Ther 2011;9:329-40.

2. Nikolic D, Katsiki N, Montalto G, et al. Lipoprotein subfractions in metabolic syndrome and obesity: clinical significance and therapeutic approaches. Nutrients 2013;5:928-48.

3. Magkos F, Mohammed BS, Mittendorfer B. Effect of obesity on the plasma lipoprotein subclass profile in normoglycemic and normolipidemic men and women. Int J Obes (Lond) 2008;32:1655-64.

4. Gerrity RG. The role of the monocyte in atherogenesis: I. Transition of blood-borne monocytes into foam cells in fatty lesions. Am J Pathol 1981;103:181-90.

5. Gerrity RG. The role of the monocyte in atherogenesis: II. Migration of foam cells from atherosclerotic lesions. Am J Pathol 1981;103:191-200.

6. Bieghs $\mathrm{V}$, Rensen $\mathrm{PC}$, Hofker $\mathrm{MH}$, et al. NASH and atherosclerosis are two aspects of a shared disease: central role for macrophages. Atherosclerosis 2012;220:287-93.

7. Wouters K, van Gorp PJ, Bieghs V, et al. Dietary cholesterol, rather than liver steatosis, leads to hepatic inflammation in hyperlipidemic mouse models of nonalcoholic steatohepatitis. Hepatology 2008;48:474-86.

8. Ioannou GN, Haigh WG, Thorning D, et al. Hepatic cholesterol crystals and crown-like structures distinguish NASH from simple steatosis. J Lipid Res 2013;54:1326-34.

9. Bieghs V, Wouters K, van Gorp PJ, et al. Role of scavenger receptor A and CD36 in diet-induced nonalcoholic steatohepatitis in hyperlipidemic mice. Gastroenterology 2010;138:2477-86, 2486 e1-3.

10. Griffin EE, Ullery JC, Cox BE, et al. Aggregated LDL and lipid dispersions induce lysosomal cholesteryl ester accumulation in macrophage foam cells. J Lipid Res 2005;46:2052-60.

11. Jerome WG, Yancey PG. The role of microscopy in understanding atherosclerotic lysosomal lipid metabolism. Microsc Microanal 2003;9:54-67.

12. Jerome WG, Lewis JC. Early atherogenesis in White Carneau pigeons. II. Ultrastructural and cytochemical observations. Am J Pathol 1985;119:210-22.

13. Haley NJ, Shio H, Fowler S. Characterization of lipid-laden aortic cells from cholesterol-fed rabbits. I. Resolution of aortic cell populations by metrizamide density gradient centrifugation. Lab Invest 1977; 37:287-96.

14. Bieghs V, Hendrikx T, van Gorp PJ, et al. The cholesterol derivative 27-hydroxycholesterol reduces steatohepatitis in mice. Gastroenterology 2013;144:167-178 e1.

15. Tessitore A, Pirozzi M, Auricchio A. Abnormal autophagy, ubiquitination, inflammation and apoptosis are dependent upon lysosomal storage and are useful biomarkers of mucopolysaccharidosis VI. Pathogenetics 2009;2:4.

16. Yan C, Lian X, Li Y, et al. Macrophage-specific expression of human lysosomal acid lipase corrects inflammation and pathogenic phenotypes in lal-/- mice. Am J Pathol 2006;169:916-26.

17. Liao G, Cheung S, Galeano J, et al. Allopregnanolone treatment delays cholesterol accumulation and reduces autophagic/lysosomal dysfunction and inflammation in Npc1-/- mouse brain. Brain Res 2009;1270:140-51.

18. Beltroy EP, Richardson JA, Horton JD, et al. Cholesterol accumulation and liver cell death in mice with Niemann-Pick type C disease. Hepatology 2005;42:886-93.

19. Jerome WG, Cash C, Webber R, et al. Lysosomal lipid accumulation from oxidized low density lipoprotein is correlated with hypertrophy of the Golgi apparatus and trans-Golgi network. J Lipid Res 1998;39:1362-71.

20. Yancey PG, Jerome WG. Lysosomal cholesterol derived from mildly oxidized low density lipoprotein is resistant to efflux. J Lipid Res 2001;42:317-27.

21. Jessup W, Mander EL, Dean RT. The intracellular storage and turnover of apolipoprotein B of oxidized LDL in macrophages. Biochim Biophys Acta 1992;1126:167-77.

22. Haley NJ, Fowler S, de Duve C. Lysosomal acid cholesteryl esterase activity in normal and lipid-laden aortic cells. J Lipid Res 1980;21:961-9.

23. Binder CJ, Horkko S, Dewan A, et al. Pneumococcal vaccination decreases atherosclerotic lesion formation: molecular mimicry between Streptococcus pneumoniae and oxidized LDL. Nature Medicine 2003;9:736-43. 
24. Holvoet P, Kritchevsky SB, Tracy RP, et al. The metabolic syndrome, circulating oxidized LDL, and risk of myocardial infarction in well-functioning elderly people in the health, aging, and body composition cohort. Diabetes 2004;53:1068-73.

25. Chalasani N, Deeg MA, Crabb DW. Systemic levels of lipid peroxidation and its metabolic and dietary correlates in patients with nonalcoholic steatohepatitis. Am J Gastroenterol 2004;99:1497-502.

26. Jerome WG, Cox BE, Griffin EE, et al. Lysosomal cholesterol accumulation inhibits subsequent hydrolysis of lipoprotein cholesteryl ester. Microsc Microanal 2008;14:138-49.

27. Maor I, Mandel H, Aviram M. Macrophage uptake of oxidized LDL inhibits lysosomal sphingomyelinase, thus causing the accumulation of unesterified cholesterol-sphingomyelin-rich particles in the lysosomes. A possible role for 7-Ketocholesterol. Arterioscler Thromb Vasc Biol 1995;15:1378-87.

28. Bordon Y. Immune regulation: lysosomes at the heart of inflammation. Nat Rev Immunol 2011; 11:502.

29. Samie MA, Xu H. Lysosomal exocytosis and lipid storage disorders. J Lipid Res 2014;55:995-1009.

30. Hannaford J, Guo H, Chen X. Involvement of cathepsins B and L in inflammation and cholesterol trafficking protein NPC2 secretion in macrophages. Obesity (Silver Spring) 2013;21:1586-95.

31. Decock J, Obermajer N, Vozelj S, et al. Cathepsin B, cathepsin H, cathepsin X and cystatin C in sera of patients with early-stage and inflammatory breast cancer. Int J Biol Markers 2008;23:161-8.

32. Sukhova GK, Zhang Y, Pan JH, et al. Deficiency of cathepsin S reduces atherosclerosis in LDL receptordeficient mice. J Clin Invest 2003;111:897-906.

33. Sukhova GK, Shi GP, Simon DI, et al. Expression of the elastolytic cathepsins $S$ and $K$ in human atheroma and regulation of their production in smooth muscle cells. J Clin Invest 1998;102:576-83.

34. Hakala JK, Oksjoki R, Laine $P$, et al. Lysosomal enzymes are released from cultured human macrophages, hydrolyze LDL in vitro, and are present extracellularly in human atherosclerotic lesions. Arterioscler Thromb Vasc Biol 2003;23:1430-6.

35. Jormsjo S, Wuttge DM, Sirsjo A, et al. Differential expression of cysteine and aspartic proteases during progression of atherosclerosis in apolipoprotein E-deficient mice. Am J Pathol 2002;161:939-45.

36. Lutgens $E$, Lutgens SP, Faber $B C$, et al. Disruption of the cathepsin $\mathrm{K}$ gene reduces atherosclerosis progression and induces plaque fibrosis but accelerates macrophage foam cell formation. Circulation 2006;113:98-107.

37. Kitamoto S, Sukhova GK, Sun J, et al. Cathepsin L deficiency reduces diet-induced atherosclerosis in low-density lipoprotein receptor-knockout mice. Circulation 2007;115:2065-75.

38. Liao G, Yao Y, Liu J, et al. Cholesterol accumulation is associated with lysosomal dysfunction and autophagic stress in Npc1 -/- mouse brain. Am J Pathol 2007;171:962-75.

39. Alam MS, Getz M, Yi S, et al. Plasma signature of neurological disease in the monogenetic disorder Niemann-Pick Type C. J Biol Chem 2014;289:8051-66.

40. Amritraj A, Peake K, Kodam A, et al. Increased activity and altered subcellular distribution of lysosomal enzymes determine neuronal vulnerability in Niemann-Pick type C1-deficient mice. Am J Pathol 2009;175:2540-56.

41. Cluzeau CV, Watkins-Chow DE, Fu R, et al. Microarray expression analysis and identification of serum biomarkers for Niemann-Pick disease, type C1. Hum Mol Genet 2012;21:3632-46.

42. Fukuo Y, Yamashina S, Sonoue $\mathrm{H}$, et al. Abnormality of autophagic function and cathepsin expression in the liver from patients with non-alcoholic fatty liver disease. Hepatol Res 2014;44:1026-36.

43. Stancikova M, Frysak Z, Trnavsky K. Effect of colchicine on the activity of cathepsin B and D in human liver cirrhosis. Acta Med Hung 1987;44:181-8.

44. Li W, Yuan XM, Olsson AG, et al. Uptake of oxidized LDL by macrophages results in partial lysosomal enzyme inactivation and relocation. Arterioscler Thromb Vasc Biol 1998;18:177-84.

45. Sims-Robinson C, Bakeman A, Rosko A, et al. The Role of Oxidized Cholesterol in Diabetes-Induced Lysosomal Dysfunction in the Brain. Mol Neurobiol 2015.

46. Hoppe G, O'Neil J, Hoff HF, et al. Products of lipid peroxidation induce missorting of the principal lysosomal protease in retinal pigment epithelium. Biochim Biophys Acta 2004;1689:33-41.

47. Conus S, Perozzo R, Reinheckel T, et al. Caspase-8 is activated by cathepsin D initiating neutrophil apoptosis during the resolution of inflammation. J Exp Med 2008;205:685-98.

48. Chwieralski CE, Welte T, Buhling F. Cathepsin-regulated apoptosis. Apoptosis 2006;11:143-9. 
49. Guicciardi ME, Deussing J, Miyoshi H, et al. Cathepsin B contributes to TNF-alpha-mediated hepatocyte apoptosis by promoting mitochondrial release of cytochrome c. J Clin Invest 2000;106: 1127-37.

50. Rajamaki K, Lappalainen J, Oorni K, et al. Cholesterol crystals activate the NLRP3 inflammasome in human macrophages: a novel link between cholesterol metabolism and inflammation. PLoS One 2010;5:e11765.

51. Vanier MT. Niemann-Pick disease type C. Orphanet J Rare Dis 2010;5:16.

52. Jerome WG. Advanced atherosclerotic foam cell formation has features of an acquired lysosomal storage disorder. Rejuvenation Res 2006;9:245-55.

53. Zhang JR, Coleman T, Langmade SJ, et al. Niemann-Pick C1 protects against atherosclerosis in mice via regulation of macrophage intracellular cholesterol trafficking. J Clin Invest 2008;118:2281-90.

54. Bieghs V, Van Gorp PJ, Wouters K, et al. LDL receptor knock-out mice are a physiological model particularly vulnerable to study the onset of inflammation in non-alcoholic fatty liver disease. PLoS One 2012;7:e30668.

55. Rimkunas VM, Graham MJ, Crooke RM, et al. In vivo antisense oligonucleotide reduction of NPC1 expression as a novel mouse model for Niemann Pick type C- associated liver disease. Hepatology 2008;47:1504-12.

56. Sayre NL, Rimkunas VM, Graham MJ, et al. Recovery from liver disease in a Niemann-Pick type C mouse model. J Lipid Res 2010;51:2372-83.

57. Reddy JV, Ganley IG, Pfeffer SR. Clues to neuro-degeneration in Niemann-Pick type C disease from global gene expression profiling. PLoS One 2006;1:e19.

58. Zampieri S, Mellon SH, Butters TD, et al. Oxidative stress in NPC1 deficient cells: protective effect of allopregnanolone. J Cell Mol Med 2009;13:3786-96.

59. Tint GS, Pentchev $P, X u$ G, et al. Cholesterol and oxygenated cholesterol concentrations are markedly elevated in peripheral tissue but not in brain from mice with the Niemann-Pick type $C$ phenotype. J Inherit Metab Dis 1998;21:853-63.

60. Vazquez MC, del Pozo T, Robledo FA, et al. Alteration of gene expression profile in Niemann-Pick type C mice correlates with tissue damage and oxidative stress. PLoS One 2011;6:e28777.

61. Rauniyar N, Subramanian K, Lavallee-Adam M, et al. Quantitative proteomics of human NPC1I1061T mutant fibroblasts provides insights into the pathogenesis of Niemann-Pick Type C disease. Mol Cell Proteomics 2015.

62. Porter FD, Scherrer DE, Lanier $\mathrm{MH}$, et al. Cholesterol oxidation products are sensitive and specific blood-based biomarkers for Niemann-Pick C1 disease. Sci Transl Med 2010;2:56ra81.

63. Fu R, Yanjanin NM, Bianconi S, et al. Oxidative stress in Niemann-Pick disease, type C. Mol Genet Metab 2010;101:214-8.

64. Sumida Y, Nakashima T, Yoh T, et al. Serum thioredoxin levels as a predictor of steatohepatitis in patients with nonalcoholic fatty liver disease. J Hepatol 2003;38:32-8.

65. Zervas M, Somers KL, Thrall MA, et al. Critical role for glycosphingolipids in Niemann-Pick disease type C. Curr Biol 2001;11:1283-7.

66. Taylor AM, Liu B, Mari Y, et al. Cyclodextrin mediates rapid changes in lipid balance in Npc1-/- mice without carrying cholesterol through the bloodstream. J Lipid Res 2012;53:2331-42.

67. Maass F, Petersen J, Hovakimyan M, et al. Reduced cerebellar neurodegeneration after combined therapy with cyclodextrin/allopregnanolone and miglustat in NPC1: a mouse model of Niemann-Pick type C1 disease. J Neurosci Res 2015;93:433-42.

68. Lloyd-Evans E, Morgan AJ, He X, et al. Niemann-Pick disease type C1 is a sphingosine storage disease that causes deregulation of lysosomal calcium. Nat Med 2008;14:1247-55.

69. Smith D, Wallom KL, Williams IM, et al. Beneficial effects of anti-inflammatory therapy in a mouse model of Niemann-Pick disease type C1. Neurobiol Dis 2009;36:242-51.

70. Cho S, Hwang ES. Status of mTOR activity may phenotypically differentiate senescence and quiescence. Mol Cells 2012;33:597-604.

71. Lynch G, Bi X. Lysosomes and brain aging in mammals. Neurochem Res 2003;28:1725-34.

72. Robbins E, Levine EM, Eagle H. Morphologic changes accompanying senescence of cultured human diploid cells. J Exp Med 1970;131:1211-22.

73. Yuan XM, Li W, Olsson AG, et al. The toxicity to macrophages of oxidized low-density lipoprotein is mediated through lysosomal damage. Atherosclerosis 1997;133:153-61. 
74. Singh R, Kaushik S, Wang Y, et al. Autophagy regulates lipid metabolism. Nature 2009;458:1131-5.

75. Shen $D$, Wang $X, L i X$, et al. Lipid storage disorders block lysosomal trafficking by inhibiting a TRP channel and lysosomal calcium release. Nat Commun 2012;3:731.

76. Kornfeld S. Trafficking of lysosomal enzymes in normal and disease states. J Clin Invest 1986;77:1-6.

77. Ungewickell AJ, Majerus PW. Increased levels of plasma lysosomal enzymes in patients with Lowe syndrome. Proc Natl Acad Sci U S A 1999;96:13342-4.

78. Hultberg B, Isaksson A, Sjoblad S, et al. Acid hydrolases in serum from patients with lysosomal disorders. Clin Chim Acta 1980;100:33-8.

79. Bottino DA, Lopes FG, de Oliveira FJ, et al. Relationship between biomarkers of inflammation, oxidative stress and endothelial/microcirculatory function in successful aging versus healthy youth: a transversal study. BMC Geriatr 2015;15:41.

80. Gradinaru D, Borsa C, Ionescu C, et al. Oxidized LDL and NO synthesis-Biomarkers of endothelial dysfunction and ageing. Mech Ageing Dev 2015.

81. Nobili V. Non-alcoholic fatty liver disease in children and adolescents. Clin Biochem 2014;47:720.

82. Arata M, Nakajima J, Nishimata S, et al. Nonalcoholic steatohepatitis and insulin resistance in children. World J Diabetes 2014;5:917-23.

83. Giorgio V, Prono F, Graziano F, et al. Pediatric non alcoholic fatty liver disease: old and new concepts on development, progression, metabolic insight and potential treatment targets. BMC Pediatr 2013; 13:40.

84. Ovchinsky N, Lavine JE. A critical appraisal of advances in pediatric nonalcoholic Fatty liver disease. Semin Liver Dis 2012;32:317-24.

85. Wieckowska A, McCullough AJ, Feldstein AE. Noninvasive diagnosis and monitoring of nonalcoholic steatohepatitis: present and future. Hepatology 2007;46:582-9.

86. Wieckowska A, Zein NN, Yerian LM, et al. In vivo assessment of liver cell apoptosis as a novel biomarker of disease severity in nonalcoholic fatty liver disease. Hepatology 2006;44:27-33.

87. Feldstein $A E$, Wieckowska A, Lopez AR, et al. Cytokeratin-18 fragment levels as noninvasive biomarkers for nonalcoholic steatohepatitis: a multicenter validation study. Hepatology 2009;50: 1072-8.

88. Diab DL, Yerian L, Schauer $\mathrm{P}$, et al. Cytokeratin 18 fragment levels as a noninvasive biomarker for nonalcoholic steatohepatitis in bariatric surgery patients. Clin Gastroenterol Hepatol 2008;6:1249-54.

89. Tamimi TI, Elgouhari HM, Alkhouri N, et al. An apoptosis panel for nonalcoholic steatohepatitis diagnosis. J Hepatol 2011;54:1224-9.

90. Yilmaz $\mathrm{Y}$, Dolar E, Ulukaya E, et al. Soluble forms of extracellular cytokeratin 18 may differentiate simple steatosis from nonalcoholic steatohepatitis. World J Gastroenterol 2007;13:837-44.

91. Chan WK, Sthaneshwar P, Nik Mustapha NR, et al. Limited utility of plasma M30 in discriminating nonalcoholic steatohepatitis from steatosis--a comparison with routine biochemical markers. PLoS One 2014;9:e105903.

92. Cusi K, Chang Z, Harrison S, et al. Limited value of plasma cytokeratin-18 as a biomarker for NASH and fibrosis in patients with non-alcoholic fatty liver disease. J Hepatol 2014;60:167-74.

93. Mayr M, Kiechl S, Tsimikas S, et al. Oxidized low-density lipoprotein autoantibodies, chronic infections, and carotid atherosclerosis in a population-based study. J Am Coll Cardiol 2006;47: 2436-43.

94. de Geest B, Collen D. Antibodies against oxidized LDL for non-invasive diagnosis of atherosclerotic vascular disease. Eur Heart J 2001;22:1517-8.

95. Plat J, Hendrikx $\mathrm{T}$, Bieghs $\mathrm{V}$, et al. Protective role of plant sterol and stanol esters in liver inflammation: insights from mice and humans. PLoS One 2014;9:e110758.

96. Hendrikx T, Jeurissen ML, Bieghs V, et al. Hematopoietic overexpression of Cyp27a1 reduces hepatic inflammation independently of 27-hydroxycholesterol levels in Ldlr(-/-) mice. J Hepatol 2015;62: 430-6.

97. Rosenson RS. Statins in atherosclerosis: lipid-lowering agents with antioxidant capabilities. Atherosclerosis 2004;173:1-12.

98. Dong $\mathrm{Y}$, Steffen BT, Cao J, et al. Effects of fenofibrate on plasma oxidized LDL and 8-isoprostane in a sub-cohort of GOLDN participants. Atherosclerosis 2011;214:422-5.

99. Basaranoglu M, Acbay O, Sonsuz A. A controlled trial of gemfibrozil in the treatment of patients with nonalcoholic steatohepatitis. J Hepatol 1999;31:384. 
100. Laurin J, Lindor KD, Crippin JS, et al. Ursodeoxycholic acid or clofibrate in the treatment of nonalcohol-induced steatohepatitis: a pilot study. Hepatology 1996;23:1464-7.

101. Pastori D, Polimeni L, Baratta F, et al. The efficacy and safety of statins for the treatment of nonalcoholic fatty liver disease. Dig Liver Dis 2015;47:4-11.

102. Sanyal AJ, Chalasani N, Kowdley KV, et al. Pioglitazone, vitamin E, or placebo for nonalcoholic steatohepatitis. N Engl J Med 2010;362:1675-85.

103. Abdelmalek MF, Angulo $\mathrm{P}$, Jorgensen RA, et al. Betaine, a promising new agent for patients with nonalcoholic steatohepatitis: results of a pilot study. Am J Gastroenterol 2001;96:2711-7.

104. Zein CO, Lopez R, Fu X, et al. Pentoxifylline decreases oxidized lipid products in nonalcoholic steatohepatitis: new evidence on the potential therapeutic mechanism. Hepatology 2012;56:1291-9.

105. Pietu F, Guillaud O, Walter T, et al. Ursodeoxycholic acid with vitamin E in patients with nonalcoholic steatohepatitis: long-term results. Clin Res Hepatol Gastroenterol 2012;36:146-55.

106. Miller ER, 3rd, Pastor-Barriuso R, Dalal D, et al. Meta-analysis: high-dosage vitamin E supplementation may increase all-cause mortality. Ann Intern Med 2005;142:37-46.

107. Klein EA, Thompson IM, Jr., Tangen CM, et al. Vitamin E and the risk of prostate cancer: the Selenium and Vitamin E Cancer Prevention Trial (SELECT). JAMA 2011;306:1549-56.

108. van Leeuwen $M$, Kemna MJ, de Winther MP, et al. Passive immunization with hypochlorite-oxLDL specific antibodies reduces plaque volume in LDL receptor-deficient mice. PLoS One 2013;8:e68039.

109. Faria-Neto JR, Chyu KY, Li X, et al. Passive immunization with monoclonal IgM antibodies against phosphorylcholine reduces accelerated vein graft atherosclerosis in apolipoprotein E-null mice. Atherosclerosis 2006;189:83-90. 
Summary 


\section{Summary}

Chapter 1 provides a general overview regarding the physiological metabolic functions of the liver. Thereafter, NASH is introduced as a liver disease that is often referred to as the hepatic manifestation of the metabolic syndrome. A summary is provided about the epidemiology, the pathogenesis, diagnosis and therapy of NASH. Finally, the thesis aim and outline are described.

In chapter 2, the central hypothesis of this thesis is discussed in more details and oxidized cholesterol is proposed as a novel risk factor for the development of NASH. New insights in the mechanisms are given by which oxidized LDL (oxLDL) can trigger $\mathrm{NASH}$. In addition, this chapter discusses the clinical implications of oxLDL from a diagnostic and therapeutic point of view.

In chapter 3, we hypothesized that lysosomal trapping of oxLDL in Kupffer cells will lead to hepatic inflammation. To test this hypothesis, $L d l r^{-/-}$mice were injected with LDL, acLDL and oxLDL. Mice injected with oxLDL, unlike acLDL and LDL, demonstrated increased lysosomal cholesterol accumulation in the Kupffer cells. Next to that, oxLDLinjected mice had increased hepatic inflammation. These findings indicate that trapping of the oxidized form of lipoproteins inside lysosomes of KCs is associated with the development of hepatic inflammation.

In chapter 4, we hypothesized that there is a causal relationship between lysosomal cholesterol accumulation inside Kupffer cells and hepatic inflammation. Moreover, we hypothesized that oxLDL is a significant contributor to the hepatic inflammatory response. To test these hypotheses, LdIr $^{-/}$mice were depleted from their hematopoietic system and were transplanted with bone marrow from $\mathrm{Npc}^{\text {mutant }}$ mice. Due to the macrophage-deficient Npc1 protein, which is a lysosomal cholesterol transporter, these mice develop lysosomal cholesterol accumulation exclusively in the hematopoietic system. After 3 months of HFC feeding, we found that $\mathrm{Npc}^{\text {mutant }}$ transplanted mice demonstrated severe hepatic inflammation and fibrosis. Next, we showed that by elevating the anti-oxLDL antibodies, the cholesterol metabolism, lysosomal dysfunction, and hepatic inflammation were improved. We concluded that there is a direct causal link between lysosomal cholesterol accumulation inside Kupffer cells and hepatic inflammation with a specific role for oxLDL.

In chapter 5, we hypothesized that an immune response towards oxidized cholesterol will reduce hepatic inflammation during diet-induced NASH. To test this hypothesis, we immunized $L d l r^{-/}$mice with heat-inactivated pneumococci. This immunization protocol gives rise to high levels of natural occurring anti-oxLDL antibodies, due to molecular mimicry between epitopes of the Streptococcus pneumoniae bacterium and oxLDL. After treating $\mathrm{Ldll}^{-/-}$mice with these heat-inactivated pneumococci, we 
demonstrated that hepatic inflammation and plasma cholesterol levels were significantly decreased. To this end, we concluded that pneumococcal immunization could lead to a therapeutic vaccination protocol towards the prevention of NASH.

In chapter 6, we hypothesized that plasma cathepsin D, a lysosomal enzyme, correlated with severity of liver inflammation in children with NAFLD. Liver biopsies of ninety-six children were evaluated and classified as having steatosis, borderline NASH and definite NASH. In the plasma of these patients, we have found that cathepsin D levels were significantly lower in subjects with liver inflammation compared to the subjects with borderline NASH or steatosis and reached a high diagnostic accuracy. Moreover, the gradual reduction in plasma cathepsin D corresponded with an increasing severity of liver inflammation, steatosis, hepatocellular ballooning and the NAFLD activity score. We concluded that plasma cathepsin D is a useful and novel diagnostic tool to predict hepatic inflammation in children.

In chapter 7, we hypothesized that plasma cathepsin D correlated with progression and regression of NASH in adults. In contrast to our previous observations in childhood NASH, we observed increased levels of plasma cathepsin D in adults with NASH compared to adults without hepatic inflammation. Furthermore, after surgical intervention, we found a significant reduction of plasma cathepsin $D$ levels in adult NASH patients compared to the initial levels before the intervention. We concluded that a distinct pathophysiology exists between NASH in children and adults. The observation that cathepsin D levels correlated with NASH development and regression is promising and points toward implementation of plasma cathepsin $D$ in the clinical follow-up of NASH patients.

In chapter 8, the main findings of this thesis are summarized and describe the involvement of lysosomal cholesterol accumulation in driving inflammation in the context of NASH as well as atherosclerosis. In addition, this chapter discusses the challenges in improving cholesterol trafficking in macrophages in order to reduce lysosomal cholesterol accumulation and recent successful research directions.

Finally, in chapter 9, the major findings of this thesis were discussed and placed in the current status of this field. 
Samenvatting 


\section{Samenvatting}

Ten eerste werd een algemeen overzicht gegeven in hoofdstuk 1 met betrekking tot de fysiologische functies van de lever. Daarnaast werd leverontsteking, ofwel NASH, geïntroduceerd als een leveraandoening binnen het spectrum van het metabool syndroom. Ten slotte werd de epidemiologie, pathogenese, diagnose en therapie van NASH kort samengevat.

In hoofdstuk 2 werd de centrale hypothese van deze thesis in meer detail beschreven en werd geoxideerd cholesterol voorgesteld als een nieuwe risicofactor voor het ontwikkelen van NASH. Nieuwe mechanistische inzichten werden besproken in hoe geoxideerd LDL (oxLDL) NASH kan veroorzaken. Vanuit een diagnostisch en therapeutisch oogpunt werd in dit hoofdstuk ook de klinische implicaties van oxLDL bediscussieerd.

In hoofdstuk 3 hadden we de hypothese dat lysosomale stapeling van oxLDL in Kupffer cellen leverontsteking veroorzaakt. Om deze hypothese te testen, injecteerden we $L d l r^{-/}$muizen met LDL, acLDL en oxLDL. De muizen geïnjecteerd met oxLDL, vertoonden meer lysosomale cholesterol opstapeling in de Kupffer cellen en meer leverontsteking dan in de muizen geïnjecteerd met acLDL en LDL. Deze bevindingen laten een associatie zien tussen lysosomale oxLDL stapeling in de Kupffer cellen en het ontstaan van leverontsteking in $L d l r^{-/-}$muizen. Het volgende hoofdstuk richtte zich op het oorzakelijk verband tussen de twee.

In hoofdstuk 4 hypotheseerden we dat er een oorzakelijk verband bestaat tussen lysosomale cholesterol stapeling in de Kupffer cellen en leverontsteking. Bovendien, dat oxLDL een belangrijke bijdrage levert aan het ontstekingsproces in de lever.

$\mathrm{LdIr}^{-/}$muizen werden van hun hematopoietische systeem ontzien en werden getransplanteerd met beenmerg van Npc1 mutante muizen. Door de macrofaagspecifieke uitschakeling van het NPC1 eiwit, welke een lysosomale cholesterol transporter is, ontwikkelen deze muizen lysosomale cholesterol stapeling specifiek in de hematopoietische cellen. Na een hoog-vet, hoog-cholesterol dieet van 3 maanden, ontwikkelden deze $N p c 1^{\text {mutant }}$-getransplanteerde muizen ernstige leverontsteking en fibrose. Echter, door het verhogen van anti-oxLDL antilichamen, verbeterde het cholesterol metabolisme, lysosomale dysfunctie en leverontsteking in deze muizen. Hieruit hebben we geconcludeerd dat er een direct oorzakelijk verband bestaat tussen lysosomale cholesterol stapeling in de Kupffer cellen en leverontsteking, waarbij oxLDL een belangrijke rol speelt.

In hoofdstuk 5 hypotheseerden we dat een immuunrespons tegen oxLDL leverontsteking zou verminderen. Om deze hypothese te testen, hebben we $\mathrm{LdIr}^{-/}$ muizen geïmmuniseerd met hitte-geïnactiveerde pneumococci. Vanwege een 
moleculaire gelijkenis tussen de epitopen van de Streptococcus pneumoniae bacterie en oxLDL, geeft dit immunizatie protocol een sterke verhoging van natuurlijk voorkomende anti-oxLDL antilichamen. We hebben aangetoond dat zowel leverontsteking als plasma cholesterol waarden sterk gereduceerd waren in $\mathrm{Ldlr}^{-/}$ muizen na immunisatie behandeling met deze hitte-geïnactiveerde pneumococci. Hieruit hebben we geconcludeerd dat een vaccinatie met hitte-geïnactiveerde pneumococci kan dienen als preventiemiddel voor het ontstaan van NASH.

In hoofdstuk 6 hadden we de hypothese dat er een correlatie bestaat tussen plasma cathepsine $D$, een lysosomaal enzyme, en de mate van leverontsteking in kinderen met NAFLD. Lever biopten van 96 kinderen werden geclassificeerd alszijnde steatose, borderline NASH en definitief NASH. In het plasma van deze patiënten hebben we aangetoond dat cathepsine D significant lager is in NASH patiënten vergeleken met steatose of borderline NASH patiënten. De geleidelijke daling van plasma cathepsine D kwam overeen met een toenemende vorm van leverontsteking, steatose, hepatocellulaire ballooning en NAFLD activiteitsscore. We concludeerden dat plasma cathepsine $D$ een bruikbare en nieuwe diagnostische methode is om leverontsteking in kinderen te voorspellen.

In hoofdstuk 7 werd de hypothese gesteld dat er een correlatie bestaat tussen plasma cathepsine $D$ en de progressie en regressie van NASH in volwassenen. In tegenstelling tot onze voorgaande observaties in kinderen met $\mathrm{NASH}$, toonden we in 3 complementaire volwassen NASH cohorten aan dat plasma cathepsine D verhoogd is in volwassen NASH patiënten vergeleken met volwassenen zonder NASH. Bovendien vonden we een verlaging van plasma cathepsine $D$ in volwassenen met NASH die een chirurgische interventie ondergingen, vergeleken met de beginwaarden van cathepsine D voor de interventie. Hieruit hebben we geconcludeerd dat een verschillende pathofysiologie ten grondslag ligt aan het ontstaan van NASH in kinderen ten opzichte van het ontstaan van NASH in volwassenen. De observatie dat een correlatie bestaat tussen cathepsine D en NASH progressie en regressie is veelbelovend en wijst naar een implementatie van plasma cathepsine $D$ in de diagnostiek en klinische follow-up van NASH patiënten.

In hoofdstuk 8 werd de betrokkenheid van lysosomale cholesterol stapeling in macrofagen in het aandrijven van ontsteking in de context van NASH en atherosclerose (aderverkalking) beschreven. Bovendien werden in dit hoofdstuk de uitdagingen besproken die om de hoek komen kijken bij het verbeteren van het cholesterol transport in macrofagen om zodoende lysosomale cholesterol stapeling te verminderen. Tot slot werden in hoofdstuk 9 de belangrijkste bevindingen van deze thesis geplaatst in de huidige stand van zaken. 
Valorisation 


\section{Valorisation}

\section{Societal and clinical relevance}

NASH is a disease frequently associated with obesity. In Western countries it is becoming increasingly prevalent and even becomes more common in children. In a general US population of middle-aged adults approximately $12 \%$ have NASH. However, the prevalence of NASH strongly increases up to $90 \%$ in an obese population. In children, a similar trend is observed, especially when obese. The development of NASH at a young age can have severe consequences later in life, with progression into complicated liver disease and a shorter life expectancy. In line, NASH patients can also progress to other chronic liver diseases such as fibrosis, cirrhosis, liver cancer and eventually liver failure. Unfortunately, in the latter cases, the only solution is a liver transplantation. Recent studies clearly demonstrate that NASH is amongst the leading causes for a liver transplantation and, if left untreated, will progress to become the primary cause in the near future. Thus, NASH is a worldwide health concern and urgently needs adequate diagnosis and therapy. However, so far, accurate methods and treatment options for NASH are nonexistent. The successful results obtained in this thesis show novel methods to diagnose and inhibit NASH and consequently, are extremely promising to society, for example, to alleviate the economic burden related to NASH including healthcare costs. Besides NASH, these findings also hold great potential to affect a wide range of other diseases such as obesity-related diseases (i.e. diabetes and atherosclerosis) and diseases that share disease mechanisms with NASH (i.e. lysosomal cholesterol storage diseases and alcoholic fatty liver disease).

\section{Scientific relevance}

Besides the scientific value, the results found in this thesis have enormous potential clinical relevance and are of interest to foundations, clinicians, patient associations and pharmaceutical companies. To make these clinicians and the pharmaceutical industry aware of these recent discoveries and to gather novel insights from different research perspectives, we organized the European Fatty Liver Conference in Maastricht. In addition, we published these findings in scientific and clinical journals relevant to our research field, such as The American Journal of Gastroenterology, Journal of Hepatology and Hepatology and patented our research data. In order to implement our findings into daily clinical practice, investments must be made into successful valorization procedures upon close collaboration with scientists, clinicians and the pharmaceutical industry. Currently, we are seeking such external collaborations, including the pharmaceutical industry, which can help us with further implementation. The application of a novel biomarker to detect NASH or ways to inhibit NASH development could be of huge benefit to patients with hepatic 
inflammation. Eventually, this will result in an improvement of the quality of life of NASH patients but will also dramatically decrease healthcare costs.

Besides NASH, our findings are also relevant to other fields of medicine. In analogy with our observations in the liver, much of the cholesterol is also trapped inside lysosomes of macrophages during atherosclerosis and lysosomal storage diseases. Therefore, the obtained results are also expected to be of high value for future research into the diagnosis and therapy of cardiovascular diseases and lysosomal storage diseases.

\section{Novelty}

Many studies in the field of the fatty liver focus on the total amount of lipids within the liver as the trigger for hepatic inflammation. Yet, this point of view does not explain the presence of hepatic inflammation in some healthy lean subjects. Thus, while most studies investigate the total lipid amount, the current thesis focused on the different types of cholesterol and their location within the macrophage. Here, it was shown that it is the oxidized form of LDL as the main trigger $\mathrm{NASH}$, particularly when located inside the lysosomes of the Kupffer cells. Theoretically, this view is innovative and promising as it provides the basis for new prevention, diagnostic and treatment options for NASH. Instead of the prescription of lipid-lowering therapies for NASH patients, methods to prevent or lower lysosomal cholesterol accumulation inside the Kupffer cells should be tested in order to prevent and treat human NASH. From a diagnostic point of view, current non-invasive markers that are used in the clinic to detect NASH lack specificity and sensitivity to distinguish NASH from steatosis. In the current thesis, it was demonstrated that a novel non-invasive marker could help improve the detection of NASH patients before irreversible liver damage has occurred.

\section{Potential applications}

The view that lysosomal cholesterol accumulation, particularly the storage of oxLDL, inside Kupffer cells is the central trigger for hepatic inflammation makes it a unique target for the prevention, diagnosis and therapy for NASH. Findings from this thesis suggest that high levels of anti-oxLDL antibody levels are protective for NASH development. In line, a simple immunization procedure with heat-inactivated pneumococci is an easy and inexpensive way in order to give rise to high anti-oxLDL antibody levels and to prevent NASH. Such preventive immunizations are of special interest for patients at high risk for the development of NASH.

Additionally, oxLDL should be considered as a significant risk factor for NASH and to other diseases within the spectrum of the metabolic syndrome. Therefore, circulating oxLDL requires close monitoring as it can add important prognostic information about the progression and regression of $\mathrm{NASH}$ and other obesity-related diseases. The view that plasma oxLDL serves as a risk factor for NASH, makes oxLDL an ideal candidate for 
NASH therapy. Therefore, reducing the level of LDL oxidation by anti-oxidants is a promising therapeutic strategy to improve NASH.

Lysosomal dysfunction is an aspect subsequent to lysosomal cholesterol accumulation, which is followed by a modified release of lysosomal enzymes into the plasma. The obtained results from this thesis demonstrated that plasma cathepsin $D$, a lysosomal enzyme, could be used to improve non-invasive diagnosis in both children and adults with NASH. As such, plasma cathepsin D and potentially other lysosomal enzymes have the potential to reduce the amount of liver biopsies, which generally is an invasive procedure that causes a lot of discomfort and stress for the patient.

Additionally, ways to lower lysosomal cholesterol are promising in ameliorating the hepatic inflammatory response. Studies from our group clearly demonstrated that therapies aimed at stimulating lysosomal cholesterol transport into the cytoplasm are successful in reducing NASH. Future research is now ongoing to translate these potential therapies to the human situation. 
Dankwoord 


\section{Dankwoord}

Eind goed, al goed. Dit is het dan, het eindresultaat van vier jaar onderzoek! Zonder de bijdrage van allerlei mensen was dit boekje zeker niet tot stand gekomen. Daarom wil ik in dit hoofdstuk deze personen bedanken voor hun inzet, hulp en toewijding.

Allereerst mijn promotor, Ad, dank voor de snelle gang van zaken in de afrondingsfase van mijn PhD. Vervolgens wil ik ook alle leden van de beoordelingscommissie bedanken voor het nalezen en het goedkeuren van mijn thesis.

Then, I am very grateful to my co-promotor, Ronit. The first time we met I was actually surprised to see a woman showing up (after all, reading the name Ronit could also imply a male, right?). At that moment I never would have guessed that I would finish a PhD traject in your group. Committed, warmhearted, professional, caring, funny, optimistic and a true colleague. You are all of it. Many thanks for your neverending encouragement and support as a co-promotor. I am looking forward working with you in the future.

Ger, ik bewonder je om in je overvolle agenda toch een wekelijks uurtje vrij te houden voor onderzoek. Er zullen niet veel clinici zijn die dat gedurende vier jaar steevast volhouden! Eén ding wat ik zeker van jou heb geleerd is de klinische kijk op wetenschappelijke data en presentaties, iets wat wetenschappers toch vaak over het hoofd zien. Jouw bijdrage hierin was dan ook onmisbaar. Het was fijn om jou als copromotor en als mede-organisator van het congres te hebben! Onder andere door jouw bourgondische insteek was het een geslaagd congres. Op naar de volgende editie. Ik kijk uit naar de toekomstige samenwerking samen met Ronit en jou.

Dan mijn paranimfen, Niki en Tom. Niki, in 2009 zijn we samen Moleculaire Levenswetenschappen gaan studeren in Maastricht, en hebben we gedurende deze studie zelfs stage gelopen op dezelfde afdeling bij Pul. Nu is het zover dat we beiden ons doctoraat halen! Supertof dat we goed met elkaar kunnen opschieten en dat we lief en leed regelmatig met elkaar delen tijdens een koffie- of lunchpauze. Ik voel me dan ook vereerd om ook jouw paranimf te zijn binnenkort. :-) Dan Tom, onze 'benjamin' van de groep, als jouw begeleider ben je drie jaar geleden begonnen aan je laatste Master stage bij ons. Je was één van de eerste studenten die ik onder mijn hoede nam en ik wist niet goed wat ik kon verwachten. Je was een aangename verrassing en hebt me veel werk uit handen genomen! Begeleiding was zelden nodig aangezien je zelfstandig, gemotiveerd en gedreven was, en nog altijd bent. Inmiddels ben je zelf goed op weg met je eigen onderzoek bij ons en weet ik zeker dat je je PhD op een succesvolle manier zal afronden. Bedankt om mijn paranimf te zijn!

De andere leden van de 'liver-lover' groep, Patrick, Jieyi, Tim, Mike en sindskort ook Yvonne. Het was heel fijn om met jullie samen te werken! Het 'treintje' tijdens de muisopofferingen loopt lang niet zo soepel als één van jullie er niet is. Bedankt voor 
jullie bijdrage, de goede sfeer, de vele (en soms lange) wetenschappelijke discussies en de ontzettend leuke tijd! Gewoon super, ik heb er echt van genoten!

Patrick, bij jou kon ik altijd terecht met mijn praktische vragen. Met jouw kennis en labexpertise kreeg ik er vaak (figuurlijk gezien) honderd vragen voor in de plaats terug. :-) Ik wil je bedanken voor alle labhulp, van de muisexperimenten tot aan de cathepsine kits. Dr. Tim, al sinds de Master studie zo ongeveer mijn buurmankamergenoot, allebei begonnen bij Ronit als stagiaires en erna samen bij haar kunnen starten als PhD student. Ik vond het super dat ik je paranimf mocht zijn, nogmaals bedankt daarvoor! Ik wens je heel veel succes en geluk toe in Wenen met Sabrina! Dat komt helemaal goed met jullie. Wordt het overigens niet tijd dat we met de hele 'liver-lover' groep een weekend afkomen voor een Oostenrijks feestje? Mike, onze labrat, als er iets op het lab gedaan moet worden, dan wordt dat ook gedaan! Jouw praktische expertise is van onmisbare waarde en nieuwe lab uitdagingen ga je dan ook niet uit de weg. Succes met het laatste jaar van zowel jou als ook die van Monique! Kunnen we een duo-promotie verwachten? Jieyi, humble yet determined to make your projects a success. I am convinced you will have a great PhD thesis in the end. Onze laatste aanwinst (maar zeker niet de minste), Dr. Yvonne, aan energie geen gebrek. Ik heb enorm veel respect voor wat je allemaal doet! Vervolgens alle studenten en medewerkers die hun steentje hebben bijgedragen aan verscheidene projecten en tijdelijk op onze afdeling werken of hebben gewerkt; Lennart, Mandy, Joris, Nathalie, Samantha, Rick, Riina, Maria, Robbin, Kosta, Armand, Isabell, Ruby, Ilona, Magda, Albert, Paula en Mitch. Bedankt voor jullie inzet!

Veerle, bij jou ben ik begonnen als student op het pneumococcen project. Je hebt me wegwijs gemaakt op het lab en in het wereldje van leveronderzoek, mijn dank daarvoor. Het is mede dankzij deze stage dat mij heeft doen besluiten om verder te gaan in de academische onderzoekswereld.

Dan ga ik verder met de huidige collega's van de afdeling, Jan, Dietbert, Willem en Joost, bedankt voor jullie input tijdens de labchats, seminars, journal clubs en andere meetings. Petra, bedankt voor alle hulp bij de administratieve beslommeringen die ik de afgelopen jaren op jouw bordje heb gelegd, niet alleen omtrent de promotie, maar ook met allerlei andere kleinigheidjes. Dip, good luck with your continuation of your postdoc. Karen, it was truly a lot of fun to organise the Labuitje with you. If only I had a small talent of your cooking skills. It always smells delicious. Will, bedankt voor jouw bijdrage aan het Labuitje. Yilin, all the best of luck for your final PhD year. Vivian, ik weet zeker dat zonder jou het lab er heel anders uit zou zien, bedankt voor het op orde houden ervan, uiteraard ook met hulp van Will en Patrick. Marion, onze 'flexwerker', knap hoe je op zoveel verschillende locaties kan werken en toch van alle nieuws op de hoogte bent. Merci voor alle babbels en histologische hulp! Daarnaast zal ik onze gezamenlijke labuitjes, etentjes, Ladies-avondjes, koffiepauzes, de jaarlijkse pretparkbezoekjes, kartavonden, bowling avondjes, lunchpauzes, feestjes, 
wandeltochtjes, Bicky-burger middagen, high-teas, vrijdagmiddagborrels en andere uitstapjes niet vergeten! Bedankt voor de leuke en bovenal gezellige tijden!

Miranda, Dimitris en Monique, mijn huidige kamergenootjes, bedankt voor de goede sfeer op onze kamer en uiteraard heel veel succes met jullie postdoc-onderzoeken.

Aan alle voormalige collega's van onze afdeling, Emiel, Kosta en Marjo, bedankt voor jullie feedback tijdens ons macrofaagclubje. Goed dat deze meeting nog altijd plaatsvindt. Dan Marie, Yeliz, Chantal, Laura, Guus, Lauran en Ine, allen heel hartelijk dank voor de leuke tijd! Nadine, een nieuw huis in het vooruitzicht. Alvast veel plezier met het samenstellen van je nieuwe interieur! :-) Peggy, je bent goed terecht gekomen in het onderwijs, het past bij je en ik weet zeker dat je hier ook een succes van gaat maken, net zoals je van je $\mathrm{PhD}$ gemaakt hebt.

Professor David Leake, my sincere thanks for giving me the opportunity to perform research and to be part of your pleasant group in Reading. I look forward to continuing our fruitful collaboration.

Meiden van de Pul, ook voor jullie een bedankje! Met jullie wordt de kloof tussen Pul en MolGen een stuk kleiner. Leuk dat we regelmatig met elkaar afspreken, dat houden we erin! Renske, bedankt voor de gezellige koffiepauzes en ik kijk uit naar jouw feestje samen met Dennis volgend jaar...

Aan het BC Broekhin clubje, Simone, Karin, Marleen en Ivo. Supertof dat we nog (al is het soms sporadisch) contact hebben, ondanks dat we al 10 jaar van het middelbaar af zijn. Simone, aangezien we in hetzelfde schuitje zitten, was het soms even fijn om bij te praten over onderzoek, onderwijs en alles wat daarbij komt kijken.

Esther, bedankt voor jouw altijd oprechte belangstelling voor zowel mijn onderzoek als ook daarbuiten. :-)

Aan alle familieleden, bedankt voor het tonen van jullie interesse. Hopelijk biedt dit boekje enigszins inzicht in waar ik me de afgelopen jaren mee bezig heb gehouden.

In het bijzonder, pap, mam, bedankt voor alle goede zorgen! Als ik bij jullie langskom is het even relaxen, alles wordt gedaan en ik hoef even nergens aan te denken! Jorrit, Nory en Wilbert, ook jullie bedankt voor het tonen van jullie interesse tijdens deze periode.

Davy, niets is onmogelijk met jou. Zo leren we elkaar kennen en zo wonen we samen. Bedankt voor alle (grafische) hulp de afgelopen tijd! Hopelijk gaan we samen een mooie toekomst tegemoet. 
Curriculum Vitae 
CV 


\section{Curriculum Vitae}

Sofie Walenbergh was born on September $14^{\text {th }}, 1987$ in Roermond, the Netherlands. In 2005, she acquired her high school diploma at the Bisschoppelijk College Broekhin, Roermond. She started her bachelor education in Applied Sciences at the Fontys University of Applied Sciences (Eindhoven, the Netherlands) in September 2005. After conducting her thesis work for 8 months at the Radboud University Medical Center, she finalized her bachelor study in 2009. Later that year, she enrolled into the master program of Clinical Molecular Life Sciences at Maastricht University, the Netherlands. For this degree, she performed an internship at the Department of Molecular Genetics, where she started to investigate NASH. For her senior master thesis, she performed an internship at the Department of Respiratory Medicine at Maastricht University, focusing on the role of leptin in alveolar macrophages, and graduated in July 2011.

In September 2011, she started her PhD program at the Department of Molecular Genetics within the school of Nutrition and Translational Research in Metabolism (NUTRIM) under supervision of Dr. Ronit Shiri-Sverdlov, Dr. Ger Koek and Prof. Dr. Ad Masclee. The topic of her research was lysosomal cholesterol accumulation inside Kupffer cells during NASH. During this time, she mentored several master students during their internships and conducted various teaching activities as a tutor in Problem-Based Learning (PBL) sessions. She also actively attended international and national conferences, including the American Association for the Study of Liver Diseases (AASLD), the European Association for the Study of the Liver (EASL), the European Society for Pediatric Gastroenterology Hepatology and Nutrition (ESPGHAN) and the Dutch Liver Retreat (DLR). During these conferences, she presented her research both orally and by poster presentation. During her PhD she obtained several prizes including the first price for best poster presentation at the plenary meeting of the Department of Genetics and Cell Biology in Maastricht in 2013, a waived registration bursary and a full bursary for the International Liver Congress (ILC) of the EASL in Amsterdam (2013) and in Vienna, Austria (2015), respectively. In addition, she obtained the Young Investigator award at the annual ESPGHAN meeting (Amsterdam, 2015). Further, she was an active member of the organizing committee of the first European Fatty Liver Conference, which took place in March 2015 in Maastricht.

From September 2015 until present, she continues her work as a postdoctoral researcher at the Department of Molecular Genetics in Maastricht. 


\section{List of publications}

1. Walenbergh SMA, Houben $T$, Hendrikx $T$, Jeurissen $M L J$, van Gorp PJ, Vreugdenhil ACE, Adriaanse MP, Buurman WA, Hofker MH, Mosca A, Lindsey PJ, Alisi A, Liccardo D, Panera N, Koek GH, Nobili V, Shiri-Sverdlov R. Plasma cathepsin D levels: a novel tool to predict pediatric hepatic inflammation. Am J Gastroenterology, 2015. Mar;110(3):462-70. (IF: 10.76) (Nominated for Best Clinical Hepatology paper 2015 by the Dutch society for Gastroenterology and Hepatology)

2. Walenbergh SMA, Koek GH, Bieghs V, Shiri-Sverdlov R. Non-alcoholic steatohepatitis: The role of oxidized low-density lipoproteins. J Hepatol, 2013. Apr;58(4):801-810. (IF: 11.34)

3. Walenbergh SMA, Houben $T$, Hendrikx $T$, Jeurissen ML, van Gorp PJ, Vaes $N$, Olde Damink SWM, Verheyen F, Koek GH, Lütjohann D, Grebe A, Latz E, ShiriSverdlov R. Weekly treatment of two-hydroxypropyl- $\beta$-cyclodextrin improves intracellular cholesterol levels in LDL receptor knock-out mice. Int J Mol Sci, 2015. 16(9), 21056-21069. (IF: 2.86)

4. Walenbergh SMA, Shiri-Sverdlov R. Cholesterol is a significant risk factor for nonalcoholic steatohepatitis. Expert Rev Gastroenterol Hepatol, 2015. Sep 22: 1-4. (IF: 2.42)

5. Hendrikx T, Walenbergh SMA, Hofker MH, Shiri-Sverdlov R. Lysosomal cholesterol accumulation: driver on the road to inflammation during atherosclerosis and nonalcoholic steatohepatitis. Obes Rev, 2014. May;15(5):424-33. (IF: 8.00)

6. Bieghs V, Walenbergh SMA, Hendrikx T, van Gorp PJ, Verheyen F, Olde Damink SWM, Masclee AA, Koek GH, Hofker MH, Binder CJ, Shiri-Sverdlov R. Trapping of oxidized LDL in lysosomes of Kupffer cells is a trigger for hepatic inflammation. Liver Int, 2013. Aug;33(7):1056-61. (IF: 4.85)

7. Hendrikx T, Walenbergh SMA, Jeurissen ML, Houbent $T$, van Gorp PJ, Lindsey $P$, Koek GH, Kalhan S, Pihlajamäki J, Hofker MH, Shiri-Sverdlov R. Plasma IL-1 receptor antagonist levels correlate with the development of non-alcoholic steatohepatitis. Biomarkers in Medicine, 2015. Nov;9(12):1301-1309. (IF: 2.65)

8. Bieghs V, van Gorp PJ, Walenbergh SMA, Gijbels MJJ, Verheyen F, Buurman WA, Briles DE, Hofker MH, Binder CJ, Shiri-Sverdlov R. Specific immunization strategies against oxidized LDL: A novel way to reduce non-alcoholic steatohepatitis in mice. Hepatology, 2012. Sep;56(3):894-903. (IF: 11.34) (Awarded for Best Basic Hepatology paper 2012 by the Dutch Society for Gastroenterology and Hepatology) 
9. Hendrikx T, Bieghs $V$, Walenbergh SMA, van Gorp PJ, Verheyen F, Jeurissen MLJ, Steinbusch MMF, Vaes N, Binder CJ, Koek GH, Stienstra R, Netea MG, Hofker MH, Shiri-Sverdlov R. Macrophage specific caspase-1/11 deficiency protects against cholesterol crystallization and hepatic inflammation in hyperlipidemic mice. PLoS One, 2013. Dec 2;8(12):e78792. (IF: 3.23)

10. Bieghs V, Hendrikx T, van Gorp PJ, Verheyen F, Dias Guichot $Y$, Walenbergh SMA, Jeurissen ML, Gijbels MJJ, Rensen SS, Bast A, Plat J, Kalhan SC, Leitersdorf E, Hofker MH, Lütjohann D, Shiri-Sverdlov R. The cholesterol derivative 27hydroxycholesterol reduces steatohepatitis in mice. Gastroenterology, 2013. Jan;144(1):167-178. (IF: 16.72)

11. Hendrikx T, Jeurissen ML, Bieghs V, Walenbergh SMA, van Gorp PJ, Verheyen F, Houben T, Dias Guichot Y, Gijbels MJJ, Leitersdorf E, Hofker MH, Lütjohann D, Shiri-Sverdlov R. Hematopoietic overexpression of Cyp27a1 reduces hepatic inflammation independently of 27-hydroxycholesterol levels in Ldlr-/- mice. J Hepatol, 2015. Feb;62(2):430-6. (IF: 11.34)

12. Hendrikx T, Jeurissen MLJ, van Gorp PJ, Gijbels MJJ, Walenbergh SMA, Houben $T$, Pöttgens C, Stienstra R, Netea M, Hofker MH, Donners MM, Shiri-Sverdlov R. Bone marrow specific caspase-1/11 deficiency inhibits atherosclerosis development in Ldlr-/- mice. FEBS J, 2015. Mar 29. doi: 10.1111/febs.13279. (IF: 4.00)

13. Plat J, Hendrikx T, Bieghs V, Jeurissen ML, Walenbergh SMA, van Gorp PJ, de Smet $E$, Konings M, Vreugdenhil ACE, Rensen SS, Buurman WA, Greve JW, Lütjohann D, Shiri-Sverdlov R. Protective role of plant sterol and stanol esters in liver inflammation: insights from mice and humans. PLoS One, 2014. Oct 30;9(10):e110758. (IF: 3.23)

\section{Submitted/in preparation}

- Walenbergh SMA, Houben T, Rensen SS, Bieghs V, Hendrikx T, van Gorp PJ, Jeurissen MLJ, Buurman WA, Greve JWM, Plat J, Hofker MH, Kalhan S, Lindsey P, Koek GH, Shiri-Sverdlov R. Plasma cathepsin D correlates with histological classifications of fatty liver disease in adults and responds to intervention. Submitted

- Walenbergh SMA, Houben T, Hendrikx T, van Gorp PJ, Jeurissen ML, Lenders MH, Gijbels MJJ, Plat J, Hofker MH, Binder CJ, Lütjohann D, Verheyen F, Koek GH, Shiri-Sverdlov R. Lysosomal cholesterol in Kupffer cells, particularly when oxidized, contributes to murine steatohepatitis. Submitted

- Walenbergh SMA*, Houben T*, Brandsma E, Hofker MH, Shiri-Sverdlov R. Oxidized lipids and non-alcoholic steatohepatitis (Review). In preparation 
- Walenbergh SMA*, Jeurissen ML*, Gijbels MJJ, Houben T, Hendrikx T, van Gorp PJ, Binder CJ, Koek GH, Donners MPC, Shiri-Sverdlov R. Bone marrow specific Npc1-deficiency is involved in the development of atherosclerosis in $\mathrm{Ldlr}^{-/-}$mice. In preparation

- Hendrikx T, Watzenboeck M, Walenbergh SMA, Amir S, Gruber S, Jonkers D, Kalhan S, Binder CJ, Shiri-Sverdlov R. Protective effects of natural antibodies against oxidation specific epitopes against hepatic inflammation during NASH. Submitted

\section{Book chapter}

- Walenbergh SMA, Hendrikx T, Shiri-Sverdlov R. Cholesterol and liver inflammation: from mice to human. Protein purification - Principles and Trends. iConcept Press Ltd. ISBN: 978-1-922227-40-9.

\section{Oral presentations}

- October, 2015: 6th Dutch Liver Retreat, Spier, the Netherlands

Title: Plasma cathepsin D correlates with histological classifications of fatty liver disease in adults and responds to intervention.

- June, 2015: The 5th Joint Diabetes and Metabolic Research Symposium, Maastricht, the Netherlands.

Title: Plasma cathepsin D levels: a novel tool to predict pediatric hepatic inflammation.

- May, 2015: The 48th Annual Meeting of the European Society of for Paediatric Gastroenterology, Hepatology and Nutrition (ESPGHAN), Amsterdam, the Netherlands.

Title: Plasma cathepsin D levels: a novel tool to predict pediatric hepatic inflammation.

\section{(Awarded with a Young Investigator award - Full bursary price)}

- April, 2015: The International Liver Congress; The 50th Annual Meeting of the European Association for the Study of the Liver (EASL), Vienna, Austria.

Title: Lysosomal cholesterol in Kupffer cells, particularly when oxidized, contributes to murine steatohepatitis.

\section{(Awarded with a Young Investigator award - Full bursary price)}

- March, 2015: Plenary meeting 2015, Department of Genetics and Cell Biology, Maastricht University Medical Center (MUMC), the Netherlands.

Title: NASH: diagnosis and treatment.

- March, 2015: 1st European Fatty liver Conference (EFLC), Maastricht, the Netherlands.

Title: Lysosomal cholesterol in Kupffer cells, particularly when oxidized, contributes to murine steatohepatitis. 
- October, 2014: 5th Dutch Liver Retreat, Spier, the Netherlands.

Title: Plasma cathepsin D levels: a novel tool to predict pediatric hepatic inflammation.

- October, 2013: 2nd Maastricht Inflammation in the Metabolic Syndrome and Atherosclerosis (MIMSA) mini-symposium, Maastricht, the Netherlands.

Title: Plasma cathepsin D: a promising novel non-invasive marker for early-stage NASH.

- March, 2013: Dutch Experimental Gastroenterology and Hepatology Meeting (DEGH), Voorjaarscongres, Veldhoven, the Netherlands.

Title: 27-Hydroxycholesterol: a potential treatment for non-alcoholic steatohepatitis in mice.

- November, 2012: The Netherlands Lipoprotein Club meeting, Leiden, the Netherlands.

Title: Lysosomal enzymes: a novel non-invasive approach to detect hepatic inflammation in NASH.

- October, 2012: 3rd Dutch Liver Retreat, Spier, the Netherlands.

Title: 27-Hydroxycholesterol: a potential treatment for non-alcoholic steatohepatitis in mice.

- September, 2012: 5th meeting of the European Club for Liver Cell Biology, Spier, the Netherlands.

Title: Trapping of oxidized LDL in lysosomes of Kupffer cells as a trigger for hepatic inflammation.

\section{Poster presentations}

- December, 2014: The 22nd Annual Symposium School of Nutrition and Translational Research in Metabolism (NUTRIM), Maastricht, the Netherlands.

Title: Plasma cathepsin D levels: a novel to predict pediatric hepatic inflammation. (Selected as an outstanding abstract)

- December, 2013: The 21st Annual Symposium School of Nutrition and Translational Research in Metabolism (NUTRIM), Maastricht, the Netherlands.

Title: Plasma cathepsin D: a novel promising marker for non-invasive diagnosis of non-alcoholic steatohepatitis.

- April, 2013: The International Liver Congress; The 48th Annual Meeting of the European Association for the Study of the Liver (EASL), Amsterdam, the Netherlands.

Title: Lysosomal enzymes: a novel non-invasive approach to detect hepatic inflammation in NASH.

(Awarded with a Young Investigator award - Waived registration bursary price) 
- March, 2013: Dutch Experimental Gastroenterology and Hepatology Meeting (DEGH), Voorjaarscongres, Veldhoven, the Netherlands.

Title: Cathepsin D: a novel way to improve diagnosis of non-alcoholic steatohepatitis.

- January, 2013: Plenary meeting, Department of Genetics and Cell Biology, Maastricht University Medical Center (MUMC), the Netherlands.

Title: Lysosomal enzymes: a novel non-invasive approach to detect hepatic inflammation in NASH.

\section{(Awarded with the 1st poster price)}

- January, 2013: Plenary meeting, Department of Genetics and Cell Biology, Maastricht University Medical Center (MUMC), the Netherlands.

Title: 27-Hydroxycholesterol: a potential treatment for non-alcoholic steatohepatitis in mice.

- December, 2012: The 20th Annual Symposium School of Nutrition and Translational Research in Metabolism (NUTRIM), Maastricht, the Netherlands.

Title: 27-Hydroxycholesterol: a potential treatment for non-alcoholic steatohepatitis in mice.

- November, 2012: 3rd Diabetes Day, Maastricht, the Netherlands.

Title: 27-Hydroxycholesterol: a potential treatment for non-alcoholic steatohepatitis in mice.

- November, 2012: The 63rd Annual Meeting of the American Association for the Study of Liver Diseases, Boston MA, USA.

Title: The inflammasome: a new target for detection and therapy of non-alcoholic steatohepatitis.

- November, 2013: The 63rd Annual Meeting of the American Association for the Study of Liver Diseases, Boston MA, USA.

Title: Trapping of oxidized LDL in lysosomes of Kupffer cells as a trigger for hepatic inflammation.

\section{Awards}

- Young Investigator Award - Full bursary for the 48th Annual Meeting of the European Society of for Paediatric Gastroenterology, Hepatology and Nutrition (ESPGHAN), Amsterdam, the Netherlands (2015).

- Young Investigator Award - Full bursary for the EASL International Liver Congress, Vienna, Austria (2015).

- Young Investigator Award - Waived registration bursary for the EASL International Liver Congress, Amsterdam, the Netherlands (2013).

- Poster presentation award at the Plenary meeting, Department of Genetics and Cell Biology, Maastricht University Medical Center (MUMC), the Netherlands (2013). 


\section{Granted patent}

- $\quad$ Cathepsin D as a method for diagnosing of juvenile NASH (EP14188909.7)

\section{Other contributions}

- $\quad$ Organizing committee member of the $1^{\text {st }}$ European Fatty Liver Conference (EFLC) being held at March 5-6, in Maastricht, the Netherlands. (2015)

\section{Summary:}

Around 110 delegates attended from more than ten different countries. The twoday program included 19 oral presentations, 3 early-morning workshops and 1 poster session.

- Invited scientific chair of the session 'Fibrosis'.

6th Dutch Liver Retreat, Spier, the Netherlands. (2015)

- Invited scientific chair of the session 'Ex vivo liver biology \& transplantation'. 5th Dutch Liver Retreat, Spier, the Netherlands. (2014)

- Invited scientific chair of the session 'Non-alcoholic steatohepatitis'. 4th Dutch Liver Retreat, Spier, the Netherlands. (2013) 
